Die Anwendbarkeit der Plastizitätstheorie in der Bemessung von Stahlbeton

Aurelio Muttoni

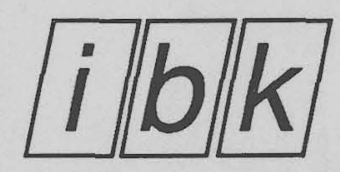

Archivexemplar:

Bild urd Grafik, Stc:.:dort $\mathbf{Z} 35.1$ 


\section{CIP-Titelaufnahme der Deutschen Bibliothek}

\section{Muttoni, Aurelio:}

Die Anwendbarkeit der Plastizitätstheorie in der Bemessung von Stahlbeton / von Aurelio Muttoni. Inst. für Baustatik u. Konstruktion, Eidgenöss. Techn. Hochsch. Zürich (ETH).-

- Basel; Boston; Berlin; Birkhäuser, 1990

(Bericht / Institut für Baustatik und Konstruktion, ETH Zürich; Nr. 176

ISBN 3-7643-2537-2

NE: Institut für Baustatik und Konstruktion 〈Zürich〉: Bericht

Nachdruck verboten.

Alle Rechte, insbesondere das der Übersetzung in fremde Sprachen und der Reproduktion auf photostatischem Wege oder durch Mikrofilm, vorbehalten. 


\title{
Die Anwendbarkeit der Plastizitătstheorie in der Bemessung von Stahlbeton
}

\author{
von \\ Dr. sc. techn. Aurelio Muttoni \\ Institut für Baustatik und Konstruktion \\ Eidgenössische Technische Hochschule Zürich (ETH)
}




\section{Vorwort}

Im Lauf der letzten 25 Jahre sind am Institut für Baustatik und Konstruktion der Eidgenössischen Technischen Hochschule (ETH) Zürich systematische Studien zur Bestimmung der Traglast von Stahlbetontragteilen mittels der Plastizitátstheorie gemacht worden. Es stelte sich dabei immer wieder die Frage, inwieweit die Annahme eines elastischen, ideal-plastischen Materialverhaltens überhaupt zu vernünftigen, d.h. mit Versuchswerten annăhernd übereinstimmenden, Resultaten führt.

In seiner Studie untersucht Herr A. Muttoni die Bedingungen und/oder Grenzen, welche bei der Anwendung der Plastizitâtstheorie in der Bemessung von Stahlbeton zu beachten sind. Nachdem nach der neuen Norm SIA 162 (1989) die Tragsicherheit anhand der statischen Methode der Plastizitătstheorie nachzuweisen ist, sollte diese Arbeit eine wertvolle Orientienung für alle Bauingenieure sein, die sich ein selbstăndiges Urteil über die entsprechenden Bemessungsverfahren bilden wollen. 


\section{Inhaltsverzeichnis}

\section{Seite}

Vorwort

1. Einleitung

2. Theoretlsche Grundlagen 3

2.1 Stoffgesetze für Materialien ohne Entfestigung 3

2.2 Die zwei Grenzwertsătze der Plastizitătstheorie 4

2.3 Stoffgesetz mit Entfestigung; Grundlagen der Entfestigungsstabilităt

2.4 Analogie zwischen Materialentfestigung und Effekte 2. Ordnung 10

2.5 Entfestigung eines Systems infolge Effekte 2. Ordnung 11

3. Materialverhalten von Stahl und Beton 13

3.1 Stahl 13

$\begin{array}{lll}3.2 & \text { Beton } & 17\end{array}$

4. Verhalten von Stahlbeton 29

4.1 Zug 29

4.2 Druck 32

$\begin{array}{lll}4.3 & \text { Biegung } & 42\end{array}$

4.4 Schub 51

5. Statisch unbestimmte Systeme mit Entfestigung 65

$\begin{array}{lll}5.1 & \text { Einführendes Beispiel } & 65\end{array}$

5.2 Systeme mit plastischen Verformungen in den Biegegelenken $\quad 71$

5.3 Systeme mit plastischen Verformungen in den Druckgliedern

5.4 Systeme mit plastischen Verformungen in den Schubgelenken 93

5.5 Plastische Bemessung statisch unbestimmter Systeme mit spröden Elementen

6. Einschrănkung der Umlagerung durch brelte Rlsse 98

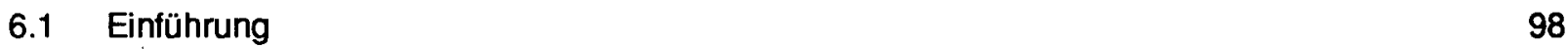

6.2 Schubbeanspruchte Balken ohne Bügelbewehrung 98

6.3 Schubbeanspruchte Druckglieder 108

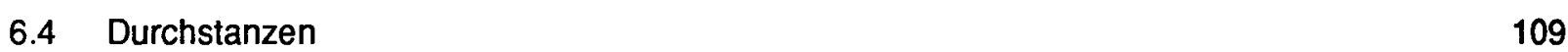

7. Verhalten plastisch bemessener Tragwerke Im Gebrauchszustand 114

7.1 Verhalten eines statisch unbestimmten Tragwerks $\quad 114$

7.2 Elastische und plastische Bemessungsmethoden 116

7.3 Einfluss der Bemessungsmethode auf das Verhalten im Gebrauchszustand 120 
Anhang A 2: Auswertung der Versuche von Richart, Brandzaeg, Brown (1929) an zylindrischen Stützenelementen mit Umschnürungsbewehrung

Anhang A 3: Auswertung der Versuche von Somes (1970)

an prismatischen Stützenelementen mit Umschnürungsbewehrung

Anhang A 4: Auswertung der Versuche von Sozen, Zwoyer, Siess (1959)

an vorgespannten Balken ohne Schubbewehrung

Anhang A 5: Auswertung der Versuche von Tomii, Sakino, Kiyohara (1981) an unbewehrten Stützenelementen 


\section{Einleitung}

Die Bemessung eines Stahlbetontragwerkes besteht darin, ein geeignetes Tragsystem zu wählen und die Betonabmessungen sowie die Bewehrung festzulegen, damit die Anforderungen in bezug auf Tragfähigkeit, Gebrauchstauglichkeit und Dauerhaftigkeit erfültt werden. Eine genügende Tragfähigkeit ist gewăhrleistet, wenn ein Tragsystem oder ein Teil desselben unter Berücksichtigung der Unsicherheiten betreffend Lasten, Festigkeiten und Bemessungsmethoden nicht einstürzt. Die Gebrauchstauglichkeit ist im allgemeinen erfüllt, wenn keine zu grossen Risse, Durchbiegungen oder Schwingungen die Qualităt und das Aussehen des Bauwerks und dessen Nutzung beeinträchtigen. Probleme wie Korrosion der Bewehrungsstăhle und Ermüdung der Tragelemente können die Dauerhaftigkeit mindern.

Zur Lossung dieser Aufgabe verfügt der Ingenieur nebst zahlreichen technologischen Kenntnissen vor allem über die Hilfsmittel der Baustatik. Letztere basieren auf drei Gruppen von Beziehungen. Die erste Gruppe beinhaltet statische Grössen, d.h. die Gleichgewichtsbeziehungen und die statischen Randbedingungen. Die zweite Gruppe umfasst kinematische Grossen, d.h. die kinematischen Beziehungen und die kinematischen Randbedingungen. Die dritte Gruppe besteht aus Stoffgesetzen, welche die statischen und die kinematischen Grössen miteinander verknüpfen. Die statischen und die kinematischen Beziehungen sind allgemein gültig. Für die Stoffgesetze müssen Annahmen getroffen werden, weil die Berücksichtigung des effektiven Materialverhaltens zu einem unverhăltnismăssigen Rechenaufwand führen würde. Aus verschiedenen Annahmen in bezug auf das Stoffgesetz resultieren die zwei wichtigsten Theorien: die Elastizitătstheorie und die Plastizitătstheorie.

Die Elastizitätstheorie setzt ein linear elastisches Verhalten der Baustoffe voraus. Die statischen und kinematischen Grössen sind somit linear verbunden. Meistens werden bei der Ermittlung der Beanspruchungen (Schnittkrăfte) homogene und isotrope Materialeigenschaften angenommen.

Die Voraussetzung eines ideal plastischen Verhaltens des Baustoffes führt zur Plastizitätstheorie. Obwohl die Formulierung der Grenzwertsătze erst in den fünfziger Jahren enfolgte (siehe z.B. die Publikationen von Prager, Hodge (1951) und Prager (1955)), sind plastische Bemessungsmethoden schon seit langem bekannt. Seit etwa hundert Jahren wird z.B. der Tragwiderstand schubbeanspruchter Balken und Scheiben mit der sogenannten Fachwerkanalogie berechnet (Hennebique (1893) und (1897); Ritter (1899); Mörsch (1908) und (1929)), und seit fast fünfzig Jahren wird die Fliessgelenklinientheorie (Johansen (1943); Gvozdev (1949)) als praktische Methode zur Ermittlung der Traglast von Platten verwendet.

In den vergangenen 25 Jahren sind zahlreiche Lösungen zur Berechnung von Stahlbetonträgern,

Stahlbetonscheiben und Stahlbetonplatten sowie von komplexen dreidimensionalen Körpern vorgeschagen worden, so dass eine einheitliche Bemessungsgrundlage vorhanden ist.

Eine gute Zusammenstellung dieser Entwicklung ist in den Arbeiten von Thürlimann, Grob, Lüchinger (1975), Thürlimann, Marti, Pralong, Ritz, Zimmerli (1983), Müller (1978), Marti (1980), Nielsen (1984) und Chen (1975) enthalten. Aufbauend auf dem unteren Grenzwertsatz der Plastizitätstheorie kann der Spannungszustand für das ganze Tragwerk oder für Teile davon ohne grossen Aufwand untersucht werden, so dass Stahlbetonstrukturen systematisch und global bemessen werden können. Dieser 
Bemessungsvorgang ist in den Arbeiten von Muttoni, Schwartz, Thürlimann (1987) und Schlaich, Weischede (1982) beschrieben.

Die Zielsetzung der vorliegenden Arbeit ist nicht die Betrachtung weiterer Anwendungsmöglichkeiten und die Entwicklung neuer Lősungen, sondern die Untersuchung der Grenzen der Anwendbarkeit der Plastizitătstheorie in der Bemessung von Stahlbetontragwerken. Die üblichen Einwendungen beziehen sich auf die Zuverlăssigkeit des Tragfähigkeitsnachweises und auf das Verhalten plastisch bemessener Tragwerke im Gebrauchszustand.

Im Kapitel 2 sind die notwendigen Grundlagen zusammengestelt. Insbesondere werden die Stoffgesetze mit Entfestigung beschrieben und eine Grösse zur Beschreibung der Sprödigkeit eines Systems eingeführt. Das Verhalten von Stahl, Beton und Stahlbeton ist in den Kapiteln 3 und 4 beschrieben. Besonders ausführlich wird das Verhalten im Entfestigungsbereich behandelt. Der Untersuchung des Einflusses der Entfestigung auf die Traglast statisch unbestimmter Tragwerke ist Kapitel 5 gewidmet. Im Kapitel 6 werden Fälle betrachtet, bei welchen die Umlagenung der inneren Krăfte durch zu breite Risse verunmöglicht wird. Es handelt sich vor allem um schubbeanspruchte Balken und Platten ohne Schubbewehrung. Die nach der Plastizitătstheorie vorgesehene Tragwirkung kann sich nicht einstellen, so dass die plastizitătstheoretische Traglast nicht erreicht werden kann.

Die Frage, ob sich plastisch bemessene Tragwerke auch im Gebrauchszustand befriedigend verhalten, wird im Kapitel 7 beantwortet. Dabei lassen sich interessante Vergleiche mit elastisch bemessenen Tragwerken ziehen.

Schliesslich werden die in den vorangehenden Kapitein gewonnenen Erkenntnisse in Form von Konstruktionsregeln verallgemeinert und zusammengestellt. 


\section{Theoretische Grundlagen}

\subsection{Stoffgesetze fur Materlalien ohne Entfestlgung}

In diesem Abschnitt werden die Beziehungen, die für die Beschreibung des mechanischen Materialverhaltens allgemein venwendet werden können, beschrieben. Die materialspezifischen Stoffgesetze für Beton und Stahl werden im Kapitel 3 erörtert.

Im Bild 2.1 sind die üblichen Stoffgesetze für einachsige Spannungszustănde dargestellt. Diese Sammlung von Spannungs-Dehnungs-Diagrammen beschrănkt sich auf die Fălle mit nicht negativer Tangentensteifigkeit. Stoffgesetze mit Entfestigung (Zunahme der Verformungen mit Abnahme der Beanspruchung) werden im Abschnitt 2.3 behandelt.

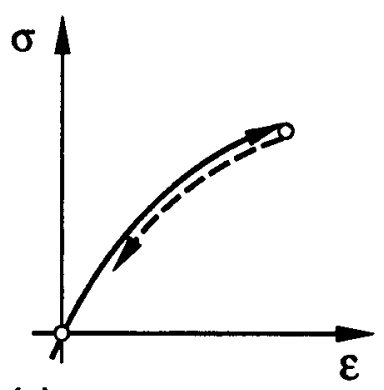

(a)

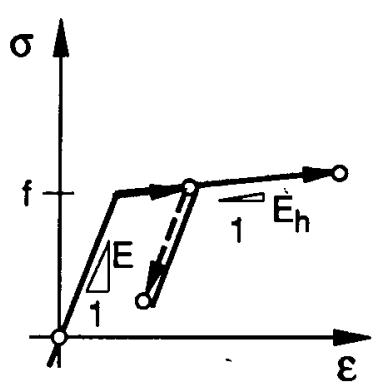

(d)

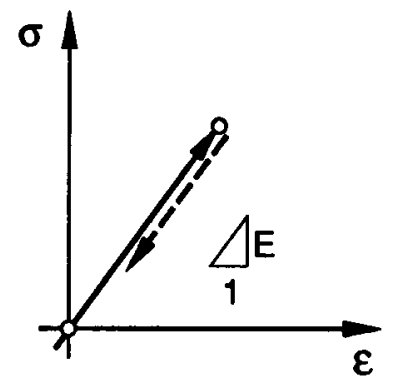

(b)

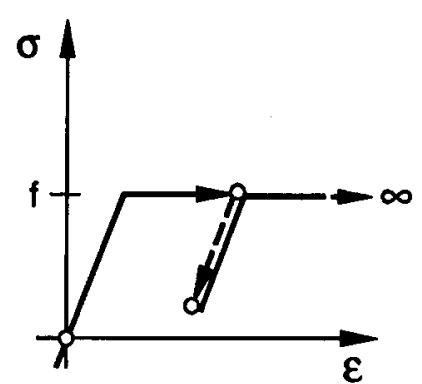

(e)

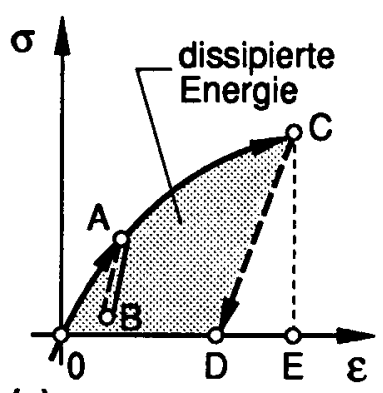

(c)

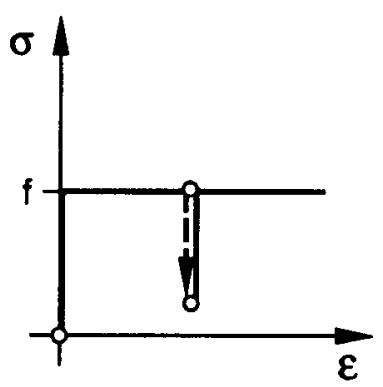

(f)

Bild 2.1: Spannungs-Dehnungs-Diagramme

Die im Bild 2.1 dargestellten Spannungs-Dehnungs-Diagramme beschreiben sowohl die Phase der monotonen Erhöhung der Beanspruchung (ausgezogene Linie) als auch jene der Entlastung (gestrichelte Linie).

Bild 2.1a zeigt ein allgemeines elastisches Stoffgesetz, wobei hier "elastisch" als Synonym für "reversibel" verwendet wird. Die Belastungs- und Entlastungskurven decken sich. Nach der Entlastung sind keine Verformungen vorhanden.

Bild 2.1b charakterisiert ein linear elastisches Stoffgesetz (auch Hookesches Gesetz genannt). Die Spannungs-Dehnungs-Beziehung wird durch den Elastizitătsmodul E als einzigen Parameter beschrieben. 
Bild 2.1c zeigt ein allgemeines plastisches Stoffgesetz, das durch die Irreversibilität des Vorgangs charakterisiert wird. Nach Belastung bis $\mathrm{C}$ und Entlastung verbleibt eine Verformung, die durch die Abszisse des Punktes $D$ gegeben ist. Die bei der Belastung gespeicherte Energie (Fläche OCE) wird bei der Entlastung nur teilweise zurückgegeben (Fläche DCE). Der Rest wird in Form von Wärme dissipiert (schraffierte Fläche).

Das im Bild 2.1d dargestellte plastisches Stoffgesetz ist charakterisiert durch seine Bilinearităt. Bis zur Grenzspannung $f$ verhălt sich ein solches Material linear elastisch. Nach Ueberschreiten dieser Grenze ist das Verhalten plastisch mit konstanter Verfestigung. Diese Phase wird durch den Verfestigungsmodul $E_{h}$ erfasst. Bei Entlastung und Wiederbelastung verhält sich das Material wieder linear elastisch-plastisch.

Bild 2.1e zeigt ein bilineares Stoffgesetz ohne Verfestigung in der plastischen Phase. Ein solches Verhalten wird als elastisch-ideal plastisch bezeichnet. Die beiden Parameter, die dieses Verhalten beschreiben, sind der Elastizitătsmodul E und die Fliesspannung f.

Wenn die plastischen Verformungen verglichen mit den elastischen gross sind, können diese vernachlässigt werden. Dieses Verhalten wird als starr-plastisch bezeichnet und ist im Bild $2.1 f$ dargestellit.

\subsection{Die zwel Grenzwertsătze der Plastizltătstheorie}

Diese zwei Theoreme dienen zur Ermittlung der Traglast von Systemen, bei welchen die Effekte 2. Ordnung (Berücksichtigung der Verformungen in den Gleichgewichtsbedingungen) vernachlässigbar sind, und welche aus einem Material mit entweder elastisch-ideal plastischem oder starr-plastischem Verhalten bestehen. Bei mehrachsigen Spannungszuständen wird das Fliessen durch eine sogenannte Fliessfigur im Spannungsraum beschrieben. Bild 2.2 a) zeigt eine solche Fliessfigur für den Fall, bei dem zwei Komponenten für die Beschreibung des Spannungstensors genügen.

Zur Herleitung der Theoreme der Plastizitătstheorie muss die Fliessbedingung zwei Bedingungen erfüllen:

- Gemäss dem ersten Axiom muss die Fliessfigur konvex sein und den Punkt enthalten, der einen spannungslosen Zustand darstellt.

- Das zweite Axiom (auch Fliessgesetz genannt) stellt den Zusammenhang zwischen Spannungszustand und Formänderung dar. Es verlangt, dass eine Spannungsänderung $\delta \sigma \underline{\sigma}$ wăhrend des Fliessens keine Arbeit beim Zuwachs der plastischen Formänderung $\delta \varepsilon_{p}$ leistet. Bild $2.2 b$ stellt diese Bedingung graphisch dar. 


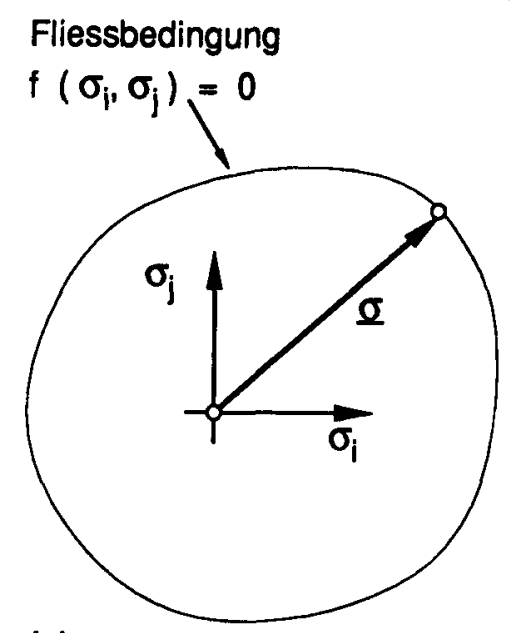

(a)

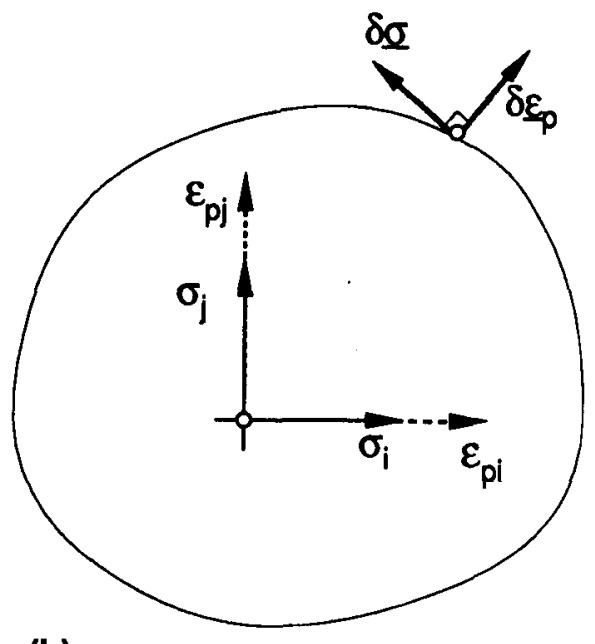

(b)

\section{Bild 2.2: Fliessbedingung und Fliessgesetz (Normalitătsbedingung)}

Beim Fliessen kann eine Spannungsănderung nur entlang der Fliessfigur stattfinden, somit ist $\delta \underline{\sigma}$ tangential zur Fliessfigur. Ein nach aussen gerichteter Vektor würde der Fliessbedingung widersprechen. Eine nach innen gerichtete Spannungsănderung $\delta \sigma$ würde zu einer Entlastung ohne plastische Verformung tâhren. Die geleistete Arbeit kann aus dem Skalarprodukt der beiden Vektoren $\delta \sigma$ mit den Spannungskomponenten $\delta \sigma_{i}, \delta \sigma_{j}$ und $\delta \varepsilon_{p}$ mit den zugehörigen Verzerrungskomponenten $\delta \varepsilon_{\text {pi' }}$ $\delta \varepsilon_{\mathrm{pj}}$ gewonnen werden. Damit die Arbeit verschwindet, müssen die Vektoren $\delta \underline{\sigma}$ im Spannungsraum und $\delta \varepsilon_{p}$ im zugehörigen Verzernungsraum zueinander senkrecht stehen. Der Vektor der plastischen Verzerrungen steht somit normal zur Fliessfigur. Dieses Axiom wird als Fliessgesetz oder auch als Normalitătsbedingung bezeichnet.

Mit diesen Axiomen lassen sich die zwei Fundamentaltheoreme der Plastizitătstheorie (auch Grenzwertsătze genannt) herleiten. Sie werden hier nur kurz enwăhnt, eine ausführliche Behandlung der theoretischen Grundlagen enthalten die Arbeiten von Prager, Hodge (1951) und Prager (1955). Die zwei Theoreme kőnnen wie folgt formuliert werden:

- Statischer Grenzwertsatz (unterer Grenzwert):

Eine Belastung $\left[Q_{S}\right]$, die zu einem Spannungsfeld gehört, das die Gleichgewichtsbedingungen und die statischen Randbedingungen erfült und auch die Fliessbedingungen nicht verletzt, ist ein unterer Grenzwert der Traglast $\left[Q_{R}\right]$ :

$$
\left[Q_{S}\right] \leq\left[Q_{R}\right]
$$

- Kinematischer Grenzwertsatz (oberer Grenzwert):

Eine Belastung $\left[Q_{K}\right]$, die mit einem Mechanismus, der die geometrischen Randbedingungen und in den plastischen Zonen das Fliessgesetz erfült, ein globales Gleichgewichtssystem bildet, ist ein oberer Grenzwert der Traglast $\left[Q_{R}\right]$ :

$$
\left[Q_{K}\right] \geq\left[Q_{R}\right]
$$


Im Kapitel 1 wurde bereits erwăhnt, dass bei der Bemessung vor allem die Anwendung des statischen Grenzwertsatzes eine direkte Ermittlung der statisch erforderlichen Widerstånde ermőglicht. Nachdem ein Spannungsfeld gewählt wird, das mit der Belastung und mit den Auflagerreaktionen im Gleichgewicht ist, können die Bemessungsgrössen so gewăhtt werden, dass die Fliessbedingungen nirgends verletzt werden.

Beim Nachweis der Tragsicherheit bestehender Konstruktionen kann die Traglast mit Hilfe der beiden Grenzwertsătze bestimmt werden. Entsprechend liegt die Traglast $\left[Q_{R}\right]$ zwischen der Last $\left[Q_{S}\right]$, die die Bedingungen des statischen Grenzwertsatzes erfült, und der Last $\left[Q_{K}\right]$, die einem Mechanismus gemäss kinematischem Grenzwertsatz entspricht. Bei der exakten Bestimmung der Traglast müssen somit die untere Schranke der Traglast (statischer Grenzwert) und die obere Schranke (kinematischer Grenzwert) so optimiert werden, dass sie sich decken. Die derart ermittelte Traglast (vollstăndige Lösung genannt) erüllt sämtliche Bedingungen beider Grenzwertsătze.

\subsection{Stoffgesetz mit Entfestigung; Grundlagen der Entfestigungsstabilităt}

Als vereinfachtes Stoffgesetz für die Beschreibung der Materialentfestigung wird zuerst eine Variante des bereits im Bild $2.1 \mathrm{~d}$ behandelten bilinearen Stoffgesetzes angenommen. Dieses ist

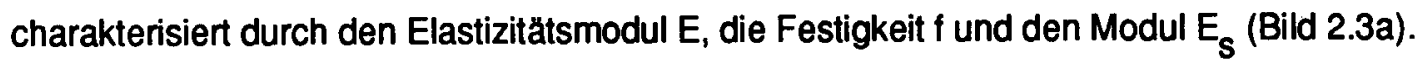

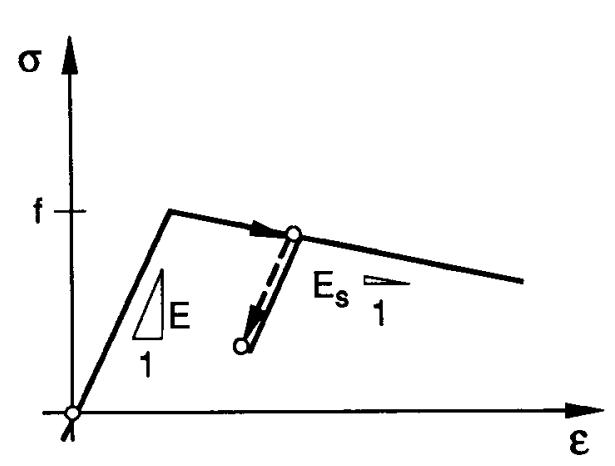

(a)

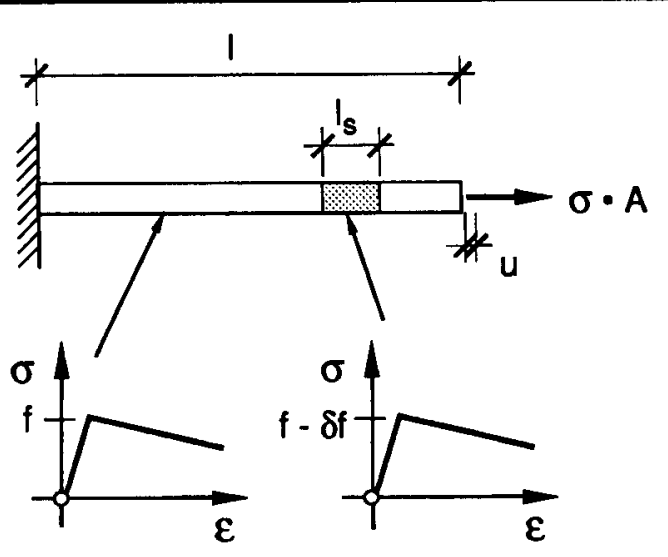

(b)

Bild 2.3: Bilineares Stoffgesetz mit Entfestigung und Anwendungsbeispiel

Die Eigenschaften eines solchen Stoffgesetzes werden anhand eines Beispiels diskutiert. Der im Bild 2.3b dargestellte Stab sei mit einer Normalkraft $A \cdot \sigma$ auf Zug beansprucht. Der Stab wird beansprucht, indem die Verlängerung u monoton erhöht wird (Wegsteuerung). Eine lastgesteuerte Beanspruchung würde in diesem Fall nach Erreichen der Festigkeit zu einer Instabilităt führen, da die Last nicht mehr erhöht werden kann. Es wird vorausgesetzt, dass die Festigkeit über die Länge nicht konstant ist. In einer Störungszone der Lănge $\iota_{s}$ ist sie um einen geringen Betrag $\delta f$ kleiner als im restlichen Teil des Stabes. In der elastischen Belastungsphase weisen beide Stabteile die gleiche Charakteristik auf, so dass die Dehnung über die Stablănge konstant verteilt ist. Nach Erreichen der Festigkeit in der Störungszone kann die Last nicht mehr erhöht werden. In dieser Zone findet eine Entfestigung statt und die Dehnung nimmt zu wăhrend die Beanspruchung abnimmt. Im restlichen Teil des Stabes kann jedoch eine ăhnliche Entfestigung nicht stattfinden, weil die 
Festigkeit nicht erreicht wurde. Eine Abnahme der Beanspruchung bewirkt somit in diesem Bereich eine Entlastung und die Dehnung nimmt ab. Wăhrend dieses Vorgangs findet, wie im Bild 2.4a dargestellt, eine Konzentration der Verformungen (Verformungslokalisienung) statt.
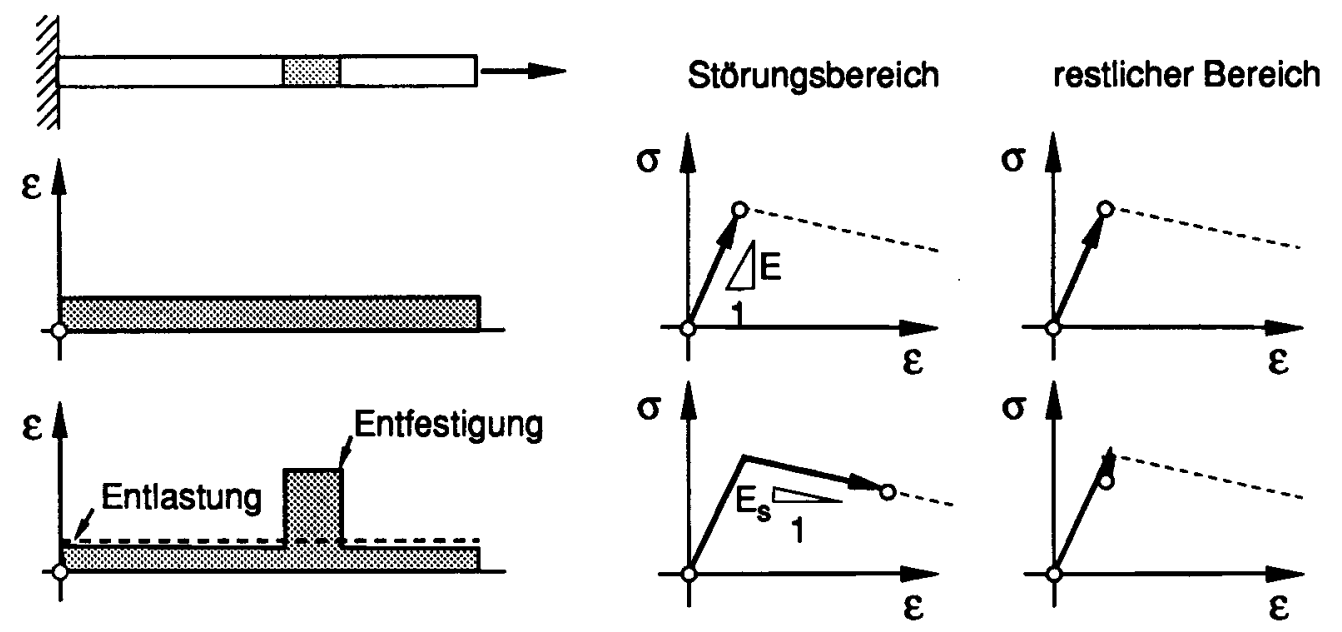

(a)

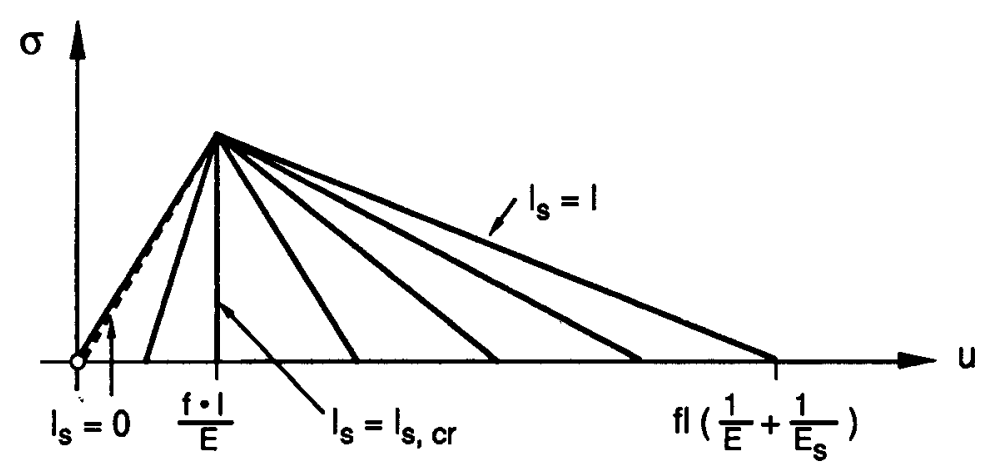

(b)

Bild 2.4: (a) Verformungslokalisierung im Entfestigungsbereich; (b) Last-Verlăngerungs-Diagramm des Stabes.

Für die Last-Venlångerungs-Charakteristik des Stabes nach Erreichen der Festigkeit in der Stórungszone gilt:

$$
\begin{array}{ll}
\text { ungestörter Bereich (Entlastung): } & u=\left(\ell-\ell_{S}\right) \cdot \frac{\sigma}{E} \\
\text { Störungsbereich (Enttestigung): } & u=\ell_{s} \cdot\left(\frac{f}{E}+\frac{f-\sigma}{E_{S}}\right)
\end{array}
$$$$
\text { so dass } u=\ell_{S} \cdot f \cdot\left(\frac{1}{E}+\frac{1}{E_{S}}\right)+\sigma \cdot\left[\frac{l}{E}-l_{s} \cdot\left(\frac{1}{E}+\frac{1}{E_{S}}\right)\right]
$$ 
Die Last-Verlăngerungs-Charakteristik des Stabes ist im Bild 2.4b für verschiedene Verhăltnisse ${ }^{\ell} s$ / graphisch dargesteltt. Je kleiner die Störungslänge $\ell_{s}$ ist, desto abrupter erfolgt der Spannungsabfall wegen Entfestigung.

Für eine kritische Lănge von ` $s$, die

$$
\ell_{S, C r}=\frac{\ell}{1+E T E_{S}}
$$

beträgt, erfolgt die Spannungsabnahme sogar ohne zusătzliche Verlăngerung des Stabes. Ein solcher Vorgang ist unstabil, auch wenn die Belastung des Stabes weggesteuert erfolgt. Der Stab bricht sofort, nachdem die maximale Last erreicht worden ist. Dies ist möglich, weil die bei der Belastung im Stab elastisch gespeicherte Energie im Entfestigungsbereich plastisch dissipiert wird. Für eine Störungslănge $\iota_{s}$, die noch kleiner als $\iota_{s, c r}$ ist, kann die gespeicherte Energie nicht mehr voll im Entfestigungsbereich dissipiert werden. Die restliche Energie wird entweder in kinetische Energie umgewandelt, so dass ein dynamisches Problem entsteht, oder sie muss wieder von der Belastungseinrichtung absorbiert werden. Nur in diesem Fall können die im Bild 2.4b dargestellten Last-Weg-Diagramme verfolgt werden.

Da die Lănge der Entfestigungszone einen entscheidenden Einfluss auf die Eigenschaften des Systems hat, ist die Kenntnis dieser Abmessung von zentraler Bedeutung. Auf diesen Sachverhalt haben schon Bazant (1976) und Schreyer, Chen (1986) hingewiesen. Wie Ottosen (1986) gezeigt hat, ist selbst der Entfestigungsvorgang in der Störungszone unstabil. Für bestimmte Werte von $E_{s} / E$ und $\ell_{s} s^{/ \ell}$ findet in dieser Zone teilweise eine Entlastung statt, so dass die Länge $\iota_{s}$ in eine Grösse übergeht, die kleiner ist als die kritische Lănge $\ell_{s, c r}$. Für die Lokalisierung der Verformungen im Störungsbereich genügt eine infinitesimale Schwächung $\delta f$. Eine probabilistische Betrachtung eines Materials mit Kontinuumseigenschaften kann jedoch zeigen, dass für $\delta$ nach null strebend auch die Länge $\ell_{s}$ zu null konvergieren muss. Dies bedeutet, dass in einem Kontinuum mit Entfestigungseigenschaften unabhăngig vom Verhăltnis $E_{s} / E$ beim Erreichen der Maximallast die elastisch gespeicherte Energie nicht dissipiert werden kann; es resultiert somit ein äusserst sprödes Verhalten. Experimente zeigen jedoch, dass für alle Materialien ein absteigender Ast vorhanden ist. Dies ist nur möglich, weil sie keine Kontinua sind. Es existiert eine materialeigene untere Grenze der Länge, über welche sich die Verformungen lokalisieren. Diese Grenze ermöglicht die Duktilität.

Das Problem der Definition der Störungslănge kann umgangen werden, indem bei den Stoffgesetzen die spezifische Verformung (Dehnung, Krümmung, Schiebung) durch eine globale Grösse (Verlängerung, Rotation, Verschiebung) ersetzt wird. Bild 2.5a zeigt das behandelte Beispiel mit der geănderten Darstellung des Stoffgesetzes.

Die Linearisierung der Entfestigungscharakteristik wird beibehalten. Das Materialgesetz kann somit mit zwei Parametem, der Festigkeit $f$ und dem Entfestigungsmodul D, beschrieben werden.

Für die Last-Verlăngerungs-Charakteristik des Stabes im Entfestigungsbereich gilt mit den neuen Bezeichnungen:

$$
u=\frac{f}{D}+\sigma \cdot\left(\frac{l}{E}-\frac{1}{D}\right)
$$




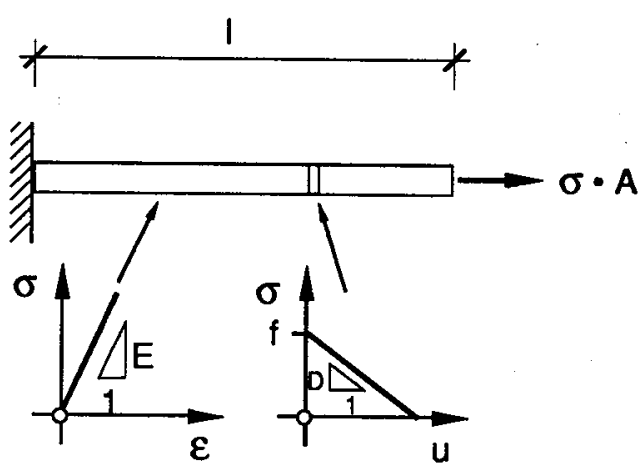

(a)

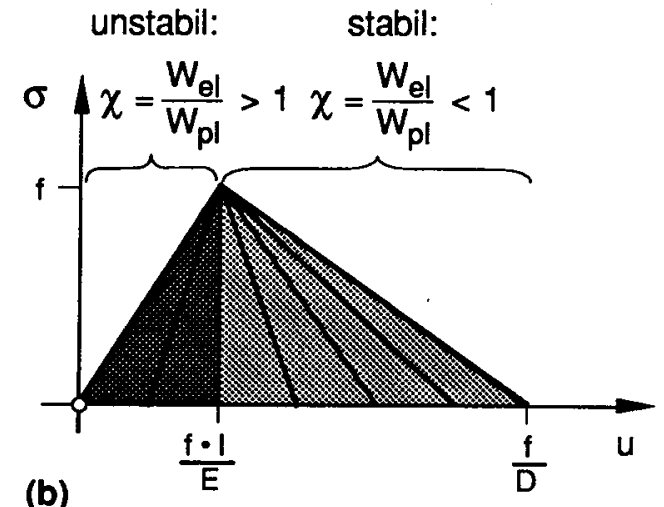

(b)

Bild 2.5: Anwendungsbeispiel mit verbesserter Darstellung des Stoffgesetzes und Last-Verlängerungs-Charakteristik des Stabes

Im Bild 2.5b ist diese Gleichung graphisch dargestellt. Die besprochenen Fälle lassen sich wie folgt unterscheiden:

- Stabiler Zustand:

Die elastisch gespeicherte Energie kann nach dem Erreichen der Maximallast plastisch voll dissipiert werden.

- Instabiler Zustand:

Plastisch kann nicht die ganze elastische Energie dissipiert werden. Ein Teil wird entweder in kinetische Energie umgewandelt, oder die Belastung muss der Abnahme des Widerstandes angepasst werden (Abnahme der Verlăngerung $u$ ).

Zur Beschreibung des Zustandes kann ein neuer Begriff eingeführt werden. Das Verhältnis zwischen potentieller (elastisch gespeicherter) und dissipierbarer Energie (plastische Dissipation) wird als Entfestigungsstabilitătsfaktor definiert:

$$
\chi=\frac{\mathrm{w}_{\mathrm{el}}}{\mathrm{W}_{\mathrm{pl}}}
$$

Mit diesem Faktor wird die Sprödigkeit des Systems beschrieben. $\chi$ kleiner als 1 zeigt einen stabilen ("duktilen") Zustand und $\chi$ grösser als 1 zeigt einen instabilen ("spröden") Zustand an. Bei nichtlinearen Stoffgesetzen sind in der Gleichung beide Energieterme durch die Ableitungen $\mathrm{dW}_{\mathrm{e}} / \mathrm{d} \sigma$ und $d W_{p /} / d \sigma$ zu ersetzen, weil der Faktor spannungsabhăngig wird.

Im behandelten Beispiel ist

$$
W_{\mathrm{el}}=A \cdot l \cdot \frac{f^{2}}{2 \mathrm{E}} \quad \text { und } \quad W_{\mathrm{pl}}=A \cdot \frac{f^{2}}{2 D}
$$

so dass der Entfestigungsstabilitătsfaktor

$$
\chi=\ell \cdot \frac{D}{E}
$$


ist. Die Gleichung enthălt drei Grössen, die die Sprödigkeit eines Systems beeinflussen. Grosse Längen, kleine Steifigkeiten sowie grosse Entfestigungsmodule sind gleichenweise für ein sprơdes Verhalten verantwortlich. Die Sprödigkeit ist somit nicht nur vom Material abhängig. Ein System, in welchem bei der Belastung eine grosse elastische Energie gespeichert wird, kann sich spröd verhalten, obwohl das Material "duktile" Eigenschaften (kleiner Entfestigungsmodul) autweist.

\subsection{Analogie zwischen Materialentfestigung und Effekte 2. Ordnung}

In der Statik werden die Gleichgewichtsbedingungen im allgemeinen am undeformierten System formuliert. Bei Systemen mit Druckkräften ist dies jedoch nicht mehr allgemein zulässig, es müssen die sogenannten Effekte 2. Ordnung berücksichtigt werden. Bild 2.6a zeigt einen starren Stab, der unten mit einer Drehfeder gehalten wird. Der Effekt 2. Ordnung kann wie folgt beschrieben werden: Eine Zunahme der Verschiebung $\Delta u$ bewirkt eine Zunahme der Beanspruchung $\Delta M$. In analoger Weise kann der Effekt der Materialentfestigung (Bild 2.6b) beschrieben werden: Eine Zunahme der Verschiebung $\Delta u$ bewirkt eine Abnahme des Widerstandes $\Delta N$.

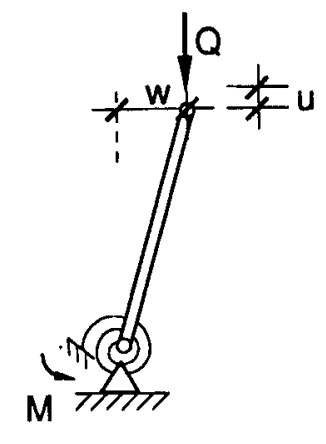

(a)

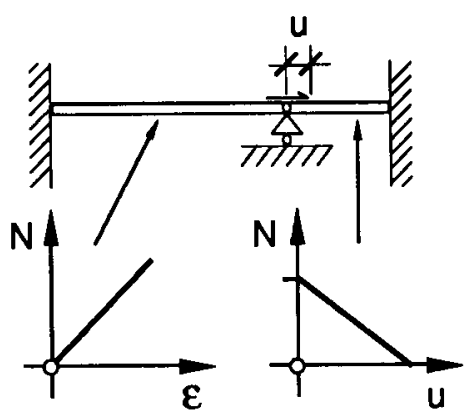

(b)

Bild 2.6: System mit Effekten 2. Ordnung und System mit Materialentfestigung

In beiden Fällen kann ein Stabilitătsproblem eintreten. Bei der geometrischen Instabilităt (2. Ordnung), wenn die Zunahme der Beanspruchung infolge Zunahme der Auslenkung der Last gleich der Zunahme des elastischen Federwiderstandes ist. Bei der Entfestigungsinstabilităt, wenn die Abnahme des Widerstandes infolge Entfestigung der Abnahme der Beanspruchung infolge Entlastung im benachbarten Stab gleicht. Im instabilen Zustand kann die Verschiebung u unbegrenzt zunehmen. Bild 2.7 zeigt die Last-Verformungs-Charakteristik des im Bild 2.6a dargestellten Systems. Als Verformung ist die Einsenkung $u$ dargestellt.

Mit einem linear elastischen Materialverhalten resultiert eine bilineare Systemcharakteristik: starr im ungeknickten Zustand, "ideal plastische" im unstabilen Zustand. Dabei wurde vorausgesetzt, dass der Stab starr ist (keine Stauchung), und dass die Beziehung Auslenkung-Rotation linear ist (Geometrie kleiner Winkel). Es ist zu bemerken, dass diese Art Instabilităt zu einem Last-Verformungs-Verhalten führt, das nur im Fall einer monotonen Lasterhöhung (Laststeuerung) instabil ist. 


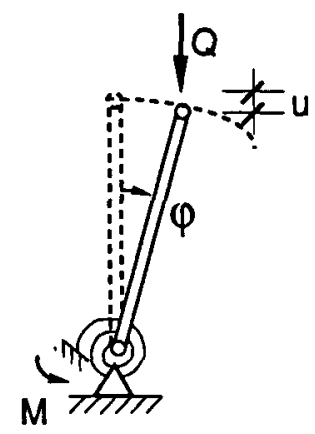

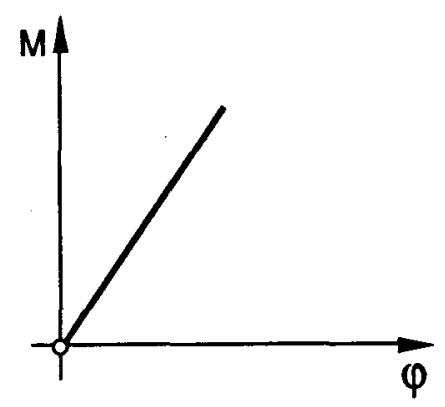

Materialverhalten (Feder)

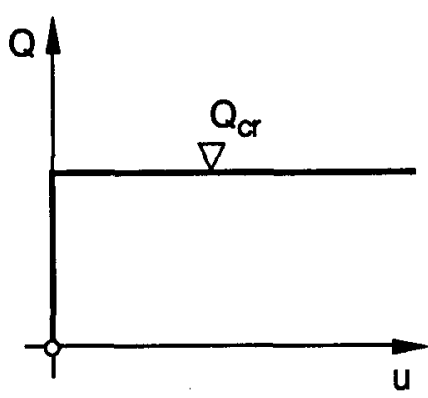

Systemverhalten

Bild 2.7: Systemverhalten bei elastischer geometrischer Stabilităt

\subsection{Entfestigung eines Systems Infolge Effekte 2. Ordnung}

Bild 2.8 zeigt die Charakteristik des elastischen-ideal plastischen Stoffgesetzes der Feder des im Bild 2.7 dargestelten Systems.

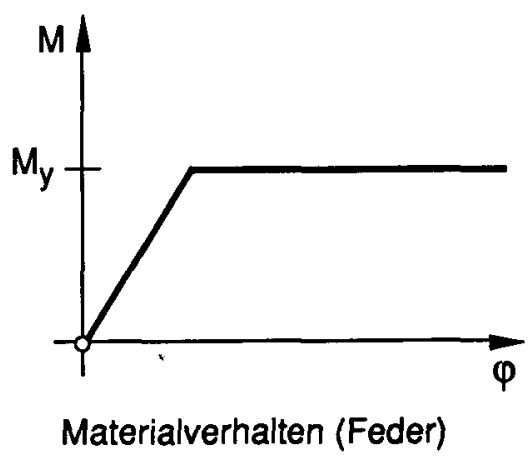

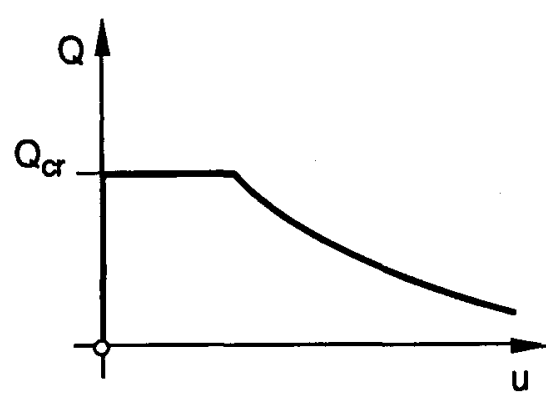

Systemverhalten

Bild 2.8: Systemverhalten bei elastisch-plastischer Beanspruchungsstabilităt

Bis zum Erreichen des plastischen Widerstandes der Feder ist das Systemverhalten bilinear. Im plastischen Bereich muss die Last mit zunehmender Verformung abnehmen, damit die Federbeanspruchung konstant bleibt. Es resultiert somit trotz ideal-plastischen Materialverhaltens eine Systementfestigung infolge von Effekten 2. Ordnung. Diese Entfestigung kann wiederum zur Entfestigungsinstabilität (instabil auch im Fall einer monotonen Erhöhung der Einsenkung) führen, wenn im System andernorts potentielle Energie elastisch gespeichert wurde. Bild 2.9a zeigt ein solches System. Der Stab verhălt sich auf Druck linear elastisch. In den Bildern 2.9b, c) und d) sind die Systemcharakteristiken für eine kleine, eine mittlere und eine grosse Lănge \& dargestellt. Bei kleinen Längen ist die im Stab gespeicherte Energie so klein, dass keine Entfestigungsinstabilităt eintritt. Bei mittleren Stablăngen kann beim Erreichen der Fliessgrenze in der Feder die im Stab gespeicherte Energie nicht dissipiert werden, so dass eine Entfestigungsinstabilităt eintritt. Das System wird bei grossen Verformungen wieder stabil. Bei grossen Stablăngen ist die kritische Last der Beanspruchungsstabilität derart gering, dass keine Entfestigungsinstabilităt eintreten kann. 


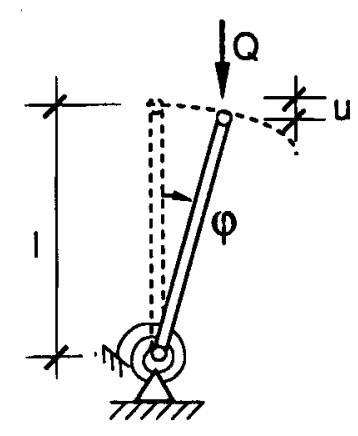

(a)

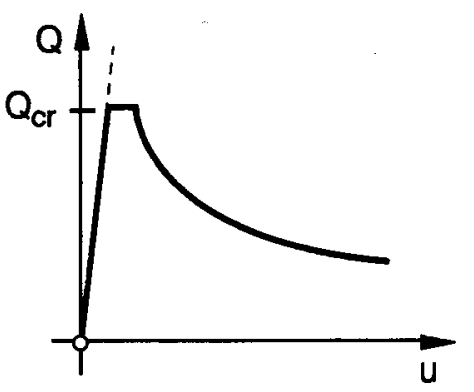

(b) 1 klein

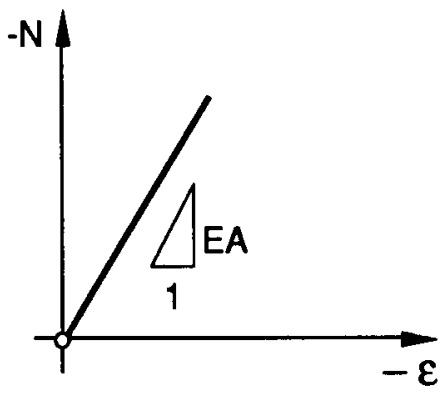

Stab

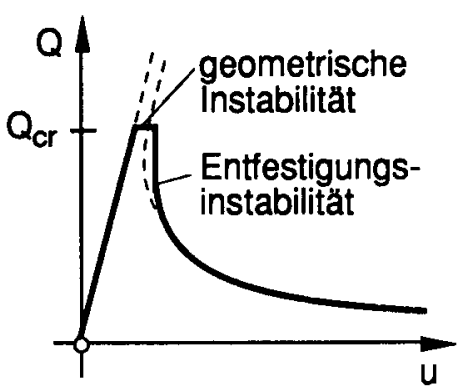

(c) 1 mittel

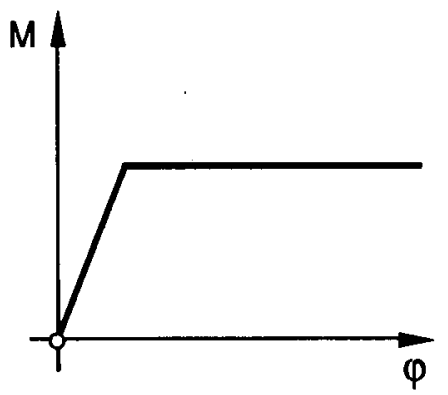

Feder

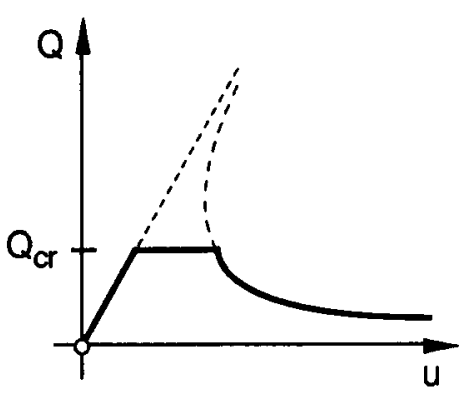

(d) I gross

Bild 2.9: Entfestigungsinstabilităt infolge von Systementfestigung bei elastischideal plastischen Materialien und grossen Verschiebungen 


\section{Materialverhalten von Stahl und Beton}

\subsection{Stahl}

Bei der Bemessung von Stahlbeton wird vereinfachend angenommen, dass die Bewehrungsstăbe nur Kräfte in Lăngsrichtung aufnehmen und daher einachsig beansprucht sind. Zuerst wird das Verhalten von auf Zug beanspruchten Bewehrungsstăhlen behandelt.

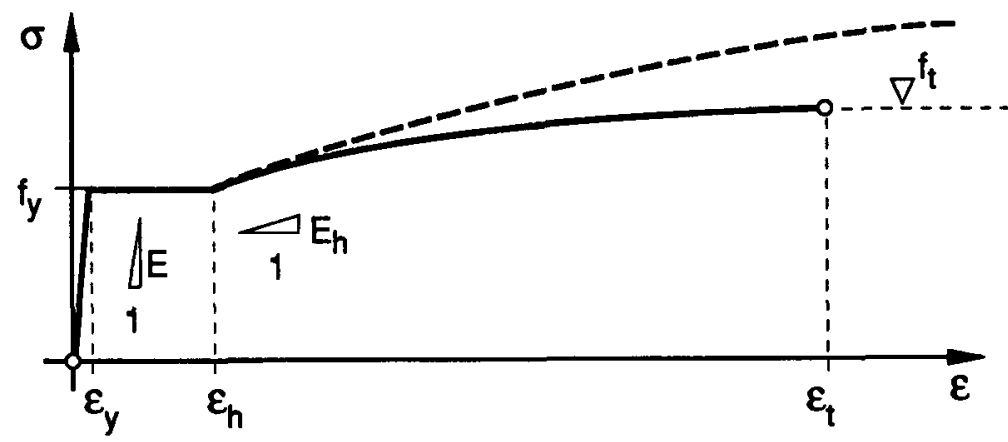

(a)

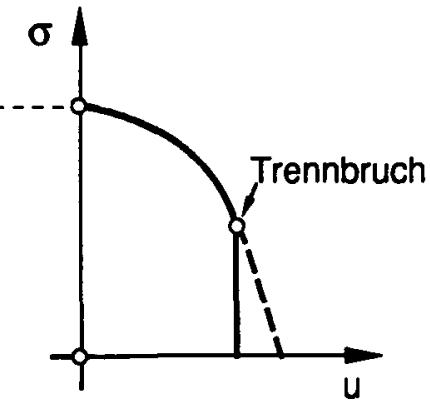

(b)

$\varepsilon_{y}=2.3 \% 0$ bis $3.0 \%$; $\quad E \quad=205^{\prime} 000 \mathrm{MPa}$;

$\varepsilon_{h}=15 \% 0$ bis $20 \% 0 ; \quad E_{h}=2^{\prime} 000 \mathrm{MPa}$ (vergüt. Stahl) bis $5^{\prime} 000 \mathrm{MPa}$ (naturh. Stahl);

$\varepsilon_{t}=100 \%$ bis $200 \%$.

Bild 3.1: (a) Spannungs-Dehnungs-Beziehung;

(b) Spannungs-Verlăngenungs-Beziehung im Entfestigungsbereich.

Im Bild 3.1 sind die Spannungs-Verformungs-Diagramme von naturhartem sowie von vergütetem Stahl dargestellt. Vier Bereiche konnen unterschieden werden:

- Ein elastischer Bereich mit linearer Charakteristik, in dem sich das Kristallgitter reversibel verformt. Die Dehnungen resultieren aus einer Aenderung des Atomabstandes. Der Elastizitătsmodul ist eine Funktion der Atomgitterstruktur und der Anziehungskrătte zwischen den Atomen. Er ist somit für alle Eisen- und Stahlarten gleich und betrăgt etwa 205'000 MPa.

- Ein plastischer Bereich, in welchem der Stahl ohne Kraftzunahme fliesst. Dieser Fliessvorgang ist mit einer Aenderung des Kristallgitters verbunden. Diese erfolgt bei Störungen der Kristallstruktur (sogenannte Versetzungen), wenn die kritische Versetzspannung erreicht wird. Da das Verhalten ideal plastisch ist (ohne Verfestigung), sind die Verformungen über die Stablänge nicht homogen. In kleinen Bereichen, sogenannten Lüdersbändern, erfolgt die Verzerrung von $\varepsilon_{y}$ bis $\varepsilon_{h}$, währenddem der restliche Stabteil keine Zusatzverformung erfăhrt. Der Fliessvorgang ist beendet, wenn sich die Lüdensbănder über die ganze Stablănge fortgepflanzt haben. 
- Ein Verfestigungsbereich, in dem die Spannung mit der Verformung wieder zunimmt. Die im Bild 3.1a dargestellten grossen Dehnungen sind somit homogen über die Stablănge verteilt. Da die Verformung praktisch ohne Volumenverănderung erfolgt, ist die grosse Dehnung in Lăngsrichtung mit einer Reduktion des Querschnittes verbunden. Es muss demnach zwischen der effektiv vorhandenen Spannung (Kraft dividiert durch effektive Querschnittsfläche (gestrichelte Linie im Diagramm)) und der nominellen Spannung (Kraft dividiert durch nominelle Querschnittsfläche (ausgezogene Linie im Diagramm)) unterschieden werden. Die maximale nominelle Spannung (Zugfestigkeit) wird erreicht, wenn die Zunahme der effektiven Spannung durch die Abnahme der effektiven Fläche kompensiert wird.

- Nach dieser Grenze ist das Verhalten des Bewehrungsstabes entfestigend, so dass sich die Verformung im sogenannten Einschnürbereich lokalisiert. Dort nimmt die effektive Querschnittsfläche rasch ab (Bild 3.1b). Diese Entfestigung führt üblicherweise zur Entfestigungsinstabilităt. Die im übrigen Stabteil bei der Entlastung treigegebene Energie kann nicht vollstăndig im Einschnürbereich dissipiert werden, so dass ein dynamisches Problem entsteht. Die Zunahme der Verformungsgeschwindigkeit und der mehrachsige Spannungszustand im Einschnürbereich führen dann im allgemeinen zu einem spröden Trennbruch.

Dank der bedeutenden Verfestigung nach dem Fliessvorgang hat eine kleine ortliche Schwăchung des Bewehrungsstabes keine Lokalisienung der Verformungen zur Folge, so dass das Verhalten duktil ist.

Eine gefährliche Lokalisierung der Verformungen erfolgt jedoch, wenn in einer örtlichen Schwăchung des Bewehrungsstabes die Zugfestigkeit $f_{t}$ erreicht wird bevor der übrige Stabteil zu fliessen beginnt. Ein solches Verhalten ist im Bild 3.2 dargestellt.

Wichtig für die Duktilităt von Stahlelementen ist somit nicht nur die grosse plastische Verformbarkeit, sondem auch das Verhältnis $\mathfrak{t}_{\mathrm{t}} f_{\mathrm{f}} \mathrm{y}$.

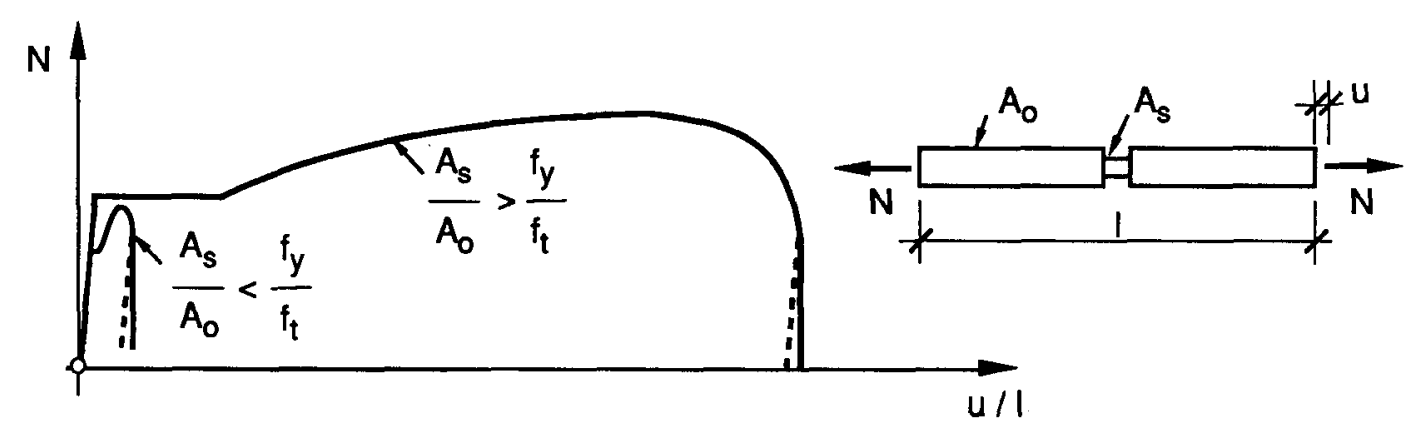

Bild 3.2: Last-Verformungs-Beziehung eines Stabes mit ortlicher Schwăchung: duktiles Verhalten mit $A_{s} / A_{o}>f_{y} / f_{t}$, sprödes Verhalten mit $A_{s} / A_{o}<f_{y} / f_{t}$.

Die Spannungs-Dehnungs-Beziehungen weiterer Stahlsorten sind im Bild 3.3 dargestellt. 


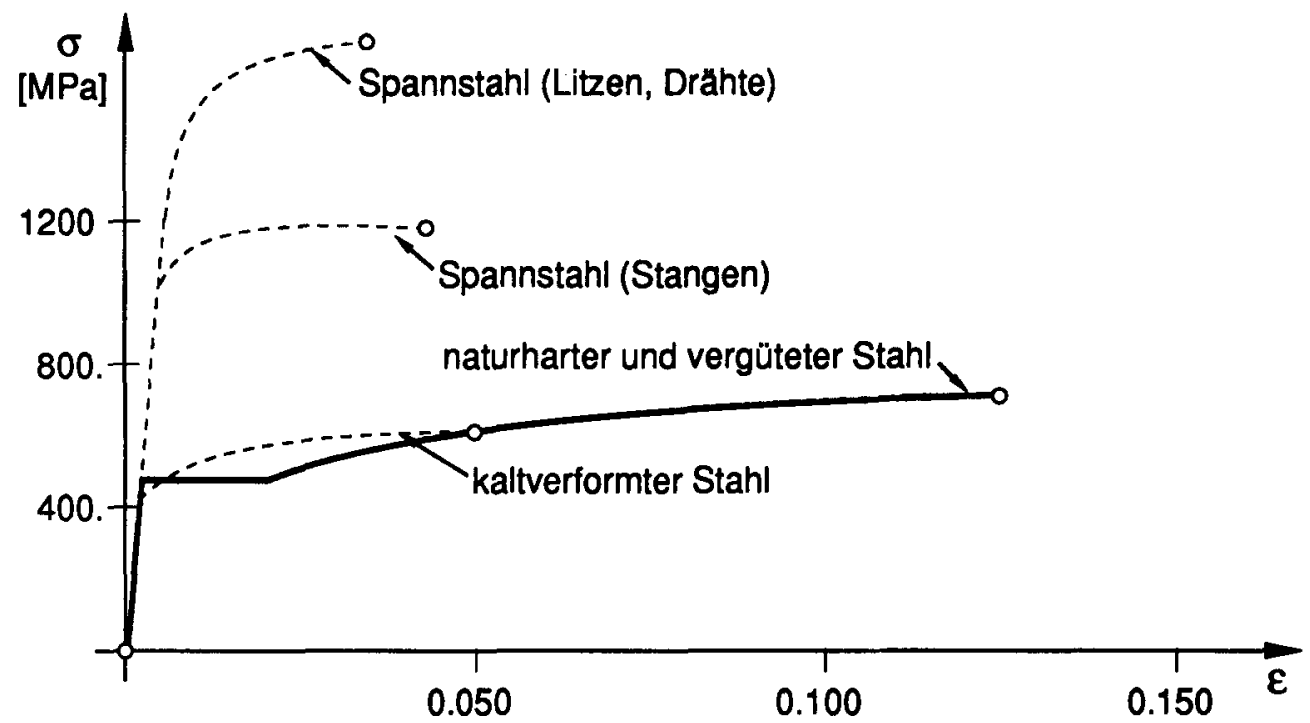

Bild 3.3: Spannungs-Dehnungs-Beziehungen verschiedener Stahlsorten

Die kaltverformten Stăhle zeigen kein Fliessplateau mehr, da das Material im Herstellungsprozess bereits bis in den Verfestigungsbereich verformt wurde. Eine ahnliche Charakteristik zeigen auch die Spannstăhle (Spanndrăhte, Litzen oder Spannstăbe).

Bei Druckbeanspruchungen sind die Spannungs-Dehnungs-Beziehungen ăhnlich wie bei Zugbeanspruchungen. Die Stauchung in Lăngsrichtung bewirkt jedoch bei Verformungen ohne Volumenănderung eine Zunahme der effektiven Fläche, so dass die nominelle Spannung grösser als die effektive ist (Bild 3.4).

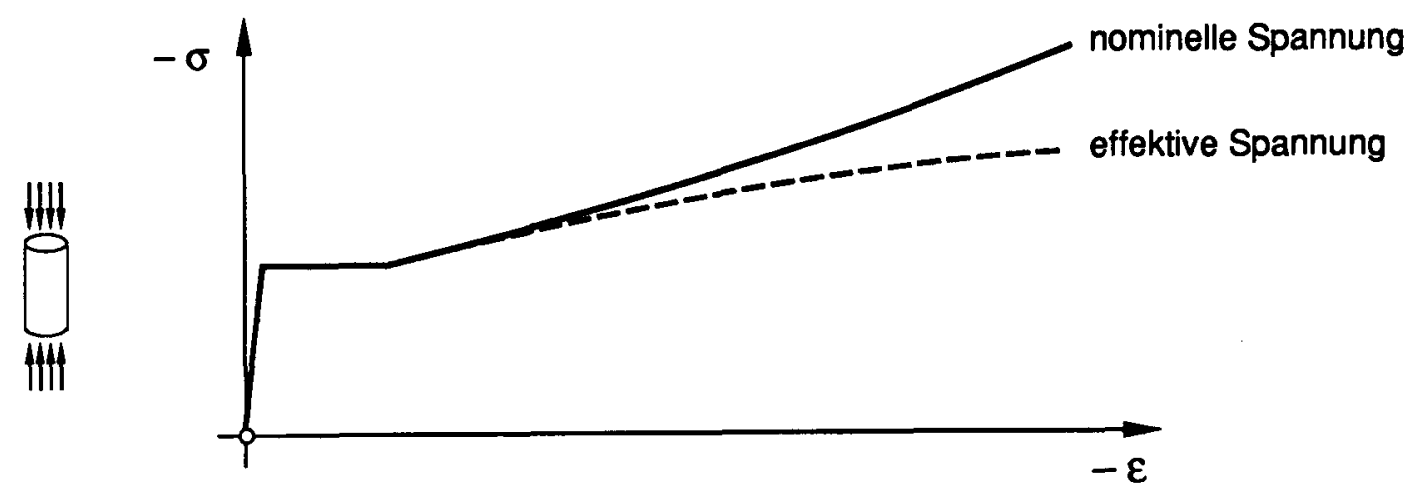

Bild 3.4: Spannungs-Dehnungs-Beziehung von druckbeanspruchtem Stahl 
Dies kann jedoch nur bei sehr gedrungenen Probekörpern festgestellt werden, da schlanke Bewehrungsstäbe knicken, bevor derart grosse Stauchungen erreicht werden. Nimmt man an, dass der Druckstab keine Exzentrizităt aułweist und nicht von Eigenspannungen und Querbelastungen beansprucht wird, kann die Stabilitătslast eines Stabes mit der Lănge \& zwischen den Einspannstellen und Durchmesser $\Phi$ mit der Eulerschen Knickgleichung bestimmt werden:

$$
\sigma_{\mathrm{Cr}}=\frac{\pi^{2} \cdot \mathrm{El}}{l_{\mathrm{Cr}}^{2} \cdot \mathrm{A}}=\frac{\pi^{2}}{4} \cdot \frac{\mathrm{E}}{(\ell / \Phi)^{2}}
$$

Wenn die Knickspannung $\sigma_{\mathrm{cr}}$ über der Elastizitătsgrenze liegt, entspricht der einzusetzende Wert von E dem Tangentenmodul, der aus dem Spannungs-Dehnungs-Diagramm herausgelesen werden kann. Im elastischen Bereich ist der Tangentenmodul gleich dem Elastizitătsmodul, im Fliessbereich sinkt er jedoch auf null. Da aber der Fliessvorgang nicht über die ganze Stablănge gleichzeitig erfolgt (Lüdersbänder), befindet sich ein Teil des Stabes im elastischen Zustand, ein weiterer Teil liegt schon im Verfestigungsbereich und ein kleiner Teil fliesst. Wie Thürlimann (1959) gezeigt hat, kőnnen im Stab verschiedene Bereiche unterschieden werden, in denen der Tangentenmodul entweder dem Elastizitătsmodul oder dem Verfestigungsmodul entspricht. Damit ein Stab vollständig bis zur Verfestigung verformt werden kann, muss die Bedingung

$$
\sigma_{c r}=\frac{\pi^{2}}{4} \cdot \frac{E_{h}}{(\ell / \Phi)^{2}} \geq f_{y}
$$

erfült werden. Für naturharten Stahl mit $E_{h} \approx 5000 \mathrm{MPa}$, für vergüteten Stahl mit $E_{h} \simeq 2000 \mathrm{MPa}$ und $f_{y}=500 \mathrm{MPa}$ resultiert somit eine Grenzschlankheit $\ell / \Phi \simeq 5$, resp. $\ell / \Phi \simeq 3$. Bei grösseren Schlankheiten knickt der Bewehrungsstab erst, wenn die bereits geflossene Zone mit einem reduzierten Tangentenmodul $E_{h}$ eine kritische Lănge erreicht. Dies ist im Bild 3.5, das die Last-Verformungs-Beziehung eines Bewehrungsstabes aus naturhartem Stahl als Funktion der Schlankheit $\ell / \Phi$ darstellt, ersichtlich.

Bei noch grösseren Schlankheiten, $(\ell / \Phi>\pi / 2 \sqrt{E / f})$, knickt der Stab bevor die Fliessgrenze erreicht wird. Wie im Abschnitt 2.4 gezeigt wurde, entfestigt sich der Stab, sobald im geknickten Zustand ein plastisches Gelenk entsteht. Die Charakteristik dieses Zustandes (Bild 3.5) wurde unter Annahme eines elastischen-ideal plastischen Verlaufs des Momenten-Krümmungs-Diagrammes ermittelt. Aehnlich ermittelte Kurven für gedrückte Stahlbauprofile zeigen eine gute Uebereinstimmung mit den Versuchsresultaten von De Donato (1969) und Schermann (1980), über die Han und Chen (1983) berichtet haben. 


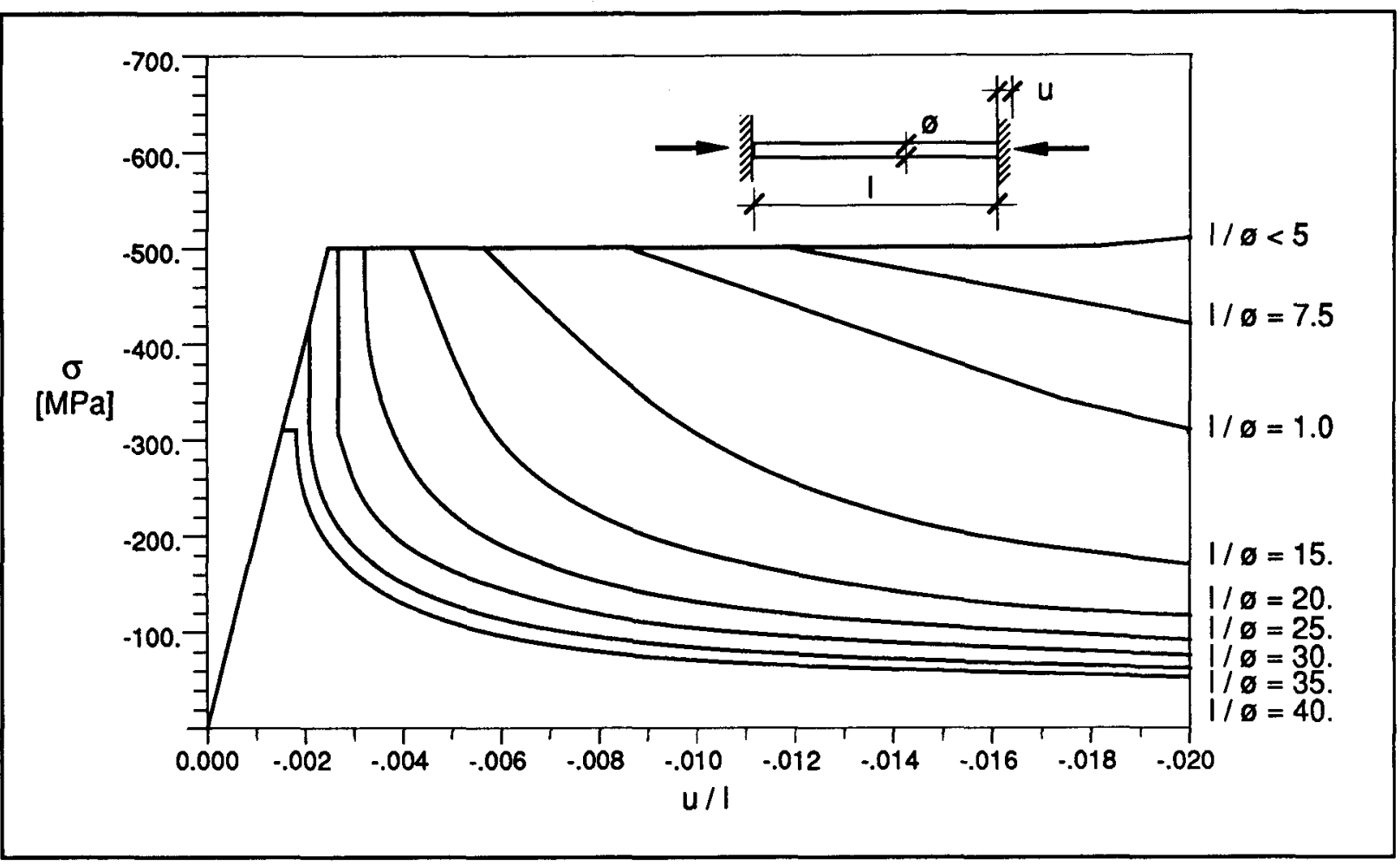

Bild 3.5: Theoretisch ermittelte Last-Verformungs-Beziehung eines gedrückten Bewehrungsstabes mit verschiedenen Schlankheiten (Material: naturharter Stahl, $f_{y}=500 \mathrm{MPa}, \varepsilon_{h}=0.018$, $E_{h}=5000 \mathrm{MPa}$ )

\subsection{Beton}

Das Last-Verformungs-Verhalten von Beton wird zuerst für einachsige Spannungszustände betrachtet. Die Untersuchung des globalen Verhaltens von zugbeanspruchtem Beton ist erst seit wenigen Jahren dank der Verwendung sehr steifer Prüfmaschinen und kleiner Prüfkörper (mit möglichst geringer Energiespeicherung) sowie moderner Beobachtungs- und Messeinrichtungen möglich geworden. Wie Cedolin, Dei Poli, lori $(1981,1983)$ mit interferometrischen Messmethoden gezeigt haben, folgt nach einer elastischen Phase, in der die Verformungen durch die Verzerrungen der Kristallgitter gegeben sind, eine teihweise irreversible Phase, in der sich die bereits vorhandenen Mikrorisse zwischen Zuschlagkornem und Zementmatrix offnen. Obwohl der Beton in dieser Phase noch nicht entfestigt wird (Bild 3.6a), sind diese Verformungen in einer Zone lokalisiert, wo die Festigkeit kleiner ist, oder wo die Beanspruchung infolge Spannungskonzentration in der Năhe einer Schwăchung grösser ist. Die Ausdehnung dieser Zone betrăgt im allgemeinen ein Mehrfaches (zwischen 2 und 3 ) des Grösstkorns. Eine Entfestigung findet erst statt, wenn sich ein bereits vorhandener Makroriss oder Mikrorisse in die Zementmatrix fortpflanzen. Wie Hillerborg (1983), Reinhardt (1984) sowie Cedolin, Dei Poli und lori (1986) gezeigt haben, fălt die Spannung senkrecht zum Trennriss nicht sofort auf null. Die Beziehung zwischen Spannung und Rissofffnung ist im Bild 3.6b dargesteltt. 
Aus Bild 3.6 geht hervor, dass beim Bruchvorgang sehr wenig Energie dissipiert wird. Der im Abschnitt 2.3 definierte Entfestigungsstabilitătsfaktor

$$
\chi=\ell \cdot \frac{D}{E}
$$

ist somit auch für kleine Lången gross. Wird

$$
D=\frac{f}{c t}
$$

angenommen (gestrich. Linie Bild 3.6b), gilt für einen Beton mit $f_{c t}=3 \mathrm{MPa}$ und $E=30000 \mathrm{MPa}$ :

$$
x=\frac{\ell}{400[\mathrm{~mm}]}
$$

Prüfkörper, die lănger als $400 \mathrm{~mm}$ sind, speichern somit wăhrend der Belastung mehr Energie als im Bruchvorgang dissipiert werden kann. Dies erklärt den spröden Charakter zugbeanspruchter Betonelemente.
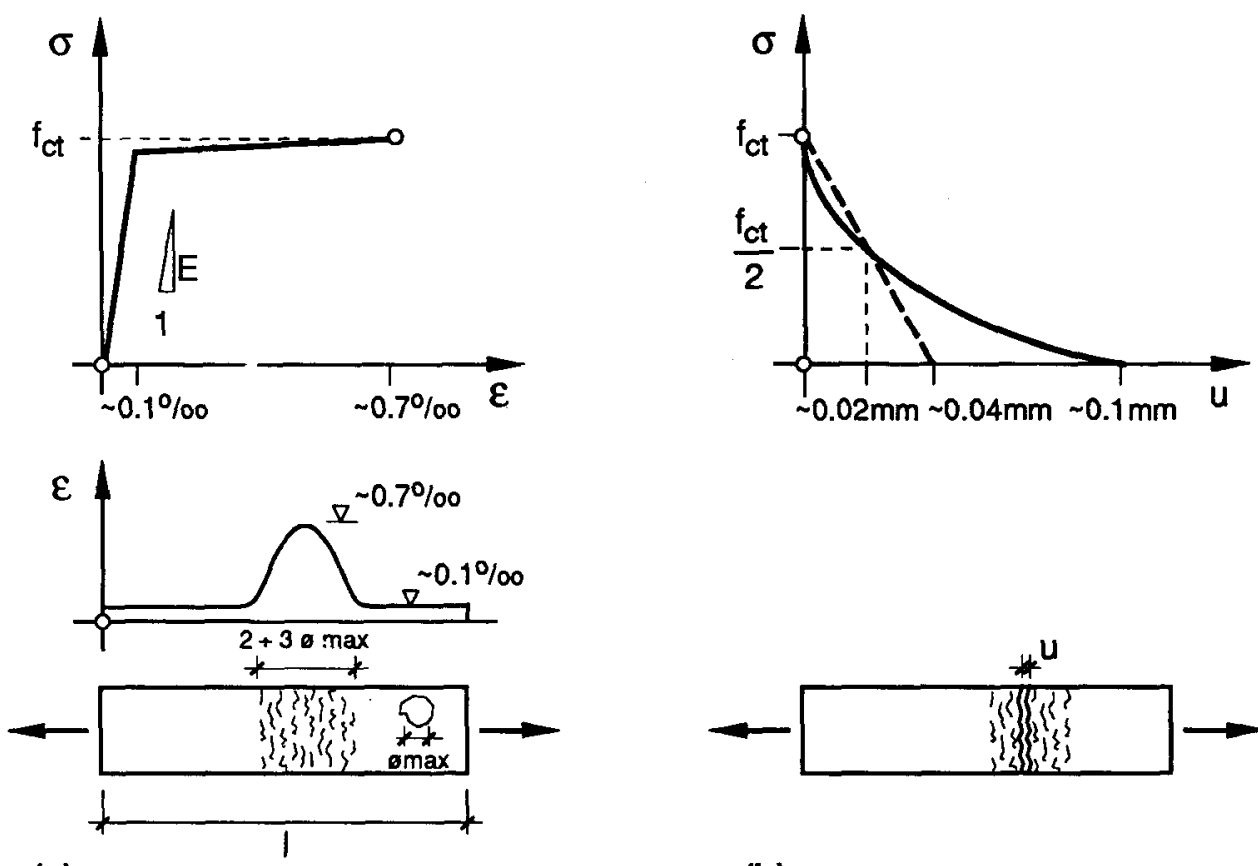

(a)

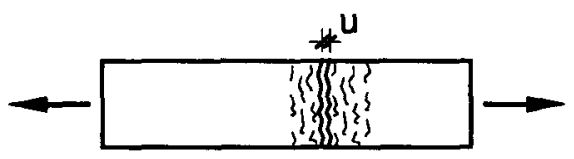

(b)

Bild 3.6: (a) Spannungs-Dehnungs-Diagramm von auf Zug beanspruchtem Beton;

(b) Spannungs-Rissőffnungs-Beziehung.

Zum besseren Verständnis der inneren Tragwirkung druckbeanspruchter Betonelemente muss berücksichtigt werden, dass die Trennschicht zwischen Zuschlagskörnern und Zementmatrix üblicherweise die schwächste Zone ist. In diesem Bereich offnen sich die bereits vorhandenen Mikrorisse (Bild 3.7a). Kritisch sind vor allem diejenigen Zonen, in denen die Trennschicht schräg zur Belastungsrichtung steht. Wenn diese Schicht hingegen senkrecht zur Belastungsrichtung steht, werden die Mikrorisse zusammengedrückt, und wenn die beiden Richtungen sich decken genügt die Kohăsion, um 
den Kraftfluss zu gewährleisten. Eine lokale Schiebung zwischen der Zementmatrix und den Zuschlagkörnern verursacht eine Umlagerung der inneren Krätte. Bild 3.7b zeigt die sich dabei einstellende neue Tragwirkung. Im kritischen Bereich ist die Hauptspannungsrichtung nicht mehr parallel zur auusseren Beanspruchungsrichtung, was eine Zunahme der Reibung in diesem Bereich verursacht. Gleichzeitig entsteht jedoch eine Querzugspannung, die die Fortpflanzung der Risse in die Zementmatrix zur Folge hat. Diese Entwicklung ist im Bild 3.7c dargestellt; der Beton spaltet sich in vertikale Lamellen. Weil in den Zonen, in denen die Trennschicht schrăg zur Belastungsrichtung steht, wiederum keine volle Kraftübertragung erfolgt, entfestigt sich der Beton. Aus der Ueberbeanspruchung der Zonen, in denen die Kraftübertragung erfolgt, entstehen neue Spaltrisse (Bild 3.7d), die zusammen mit dem Instabilwerden der Lamellen eine zusătzliche Entiestigung verursachen. Durch diese Entfestigung werden die Verformungen lokalisiert. Da der Bruchvorgang mit grossen Querdehnungen verbunden ist, wird die Abmessung der Verformungszone aus der geometrischen Vertrăglichkeit mit den ungestörten Zonen bestimmt.

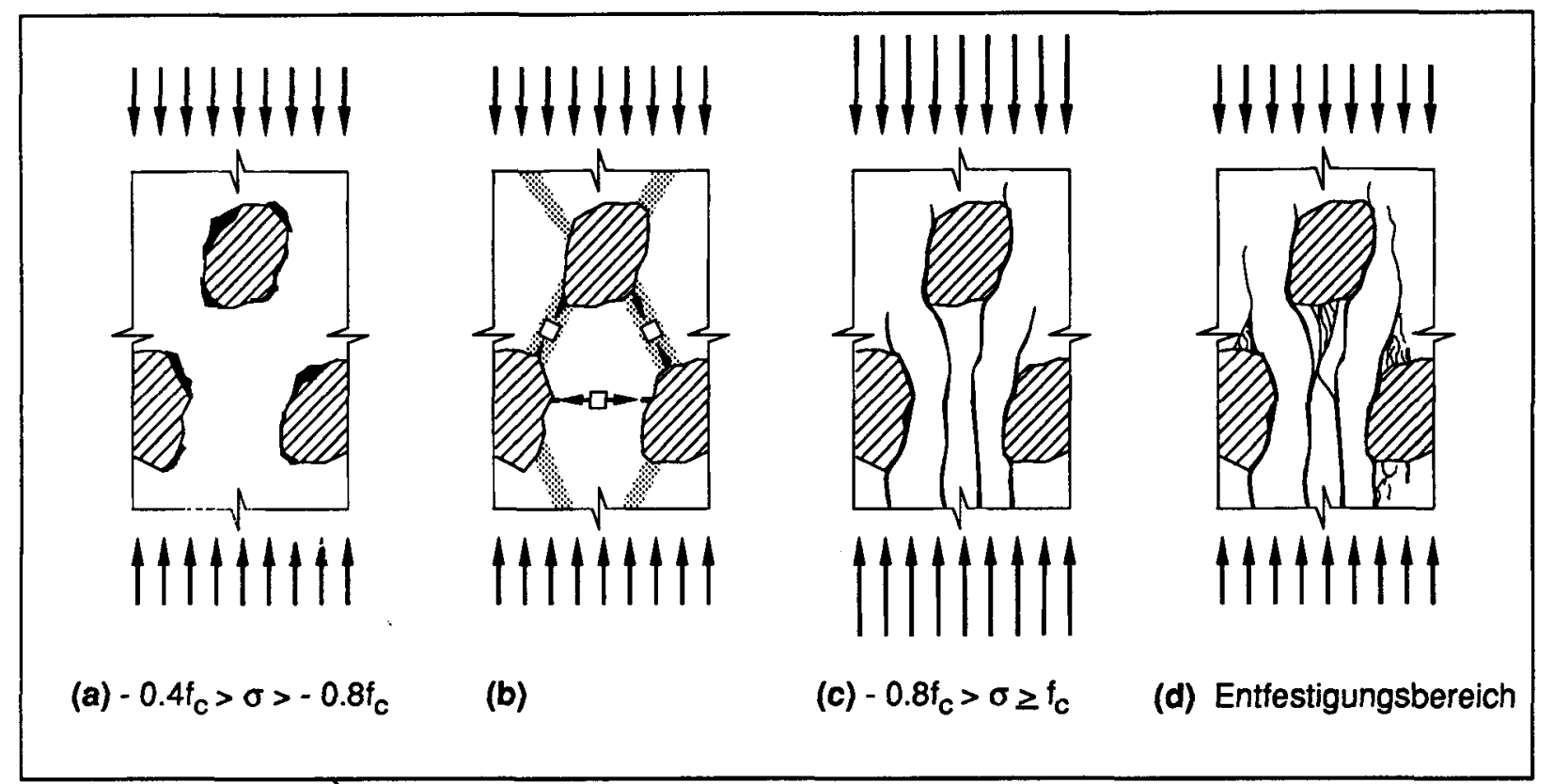

Bild 3.7: Rissbildung und innere Tragwirkung eines druckbeanspruchten Betonelementes

Bild 3.8 zeigt die Verformungsbilder zweier typischer Fălle mit den approximativen Abmessungen der Verformungszonen, wie sie experimentell ermittelt werden können.

Mit einem Verhältnis Lănge/Durchmesser $=2$ ergeben übliche Zylinderversuche die mittlere Dehnung im Verformungsbereich. Anhand der im Bild 3.9 dargestelten Spannungs-Dehnungs-Diagramme lassen sich die beschriebenen Phasen erkennen. 

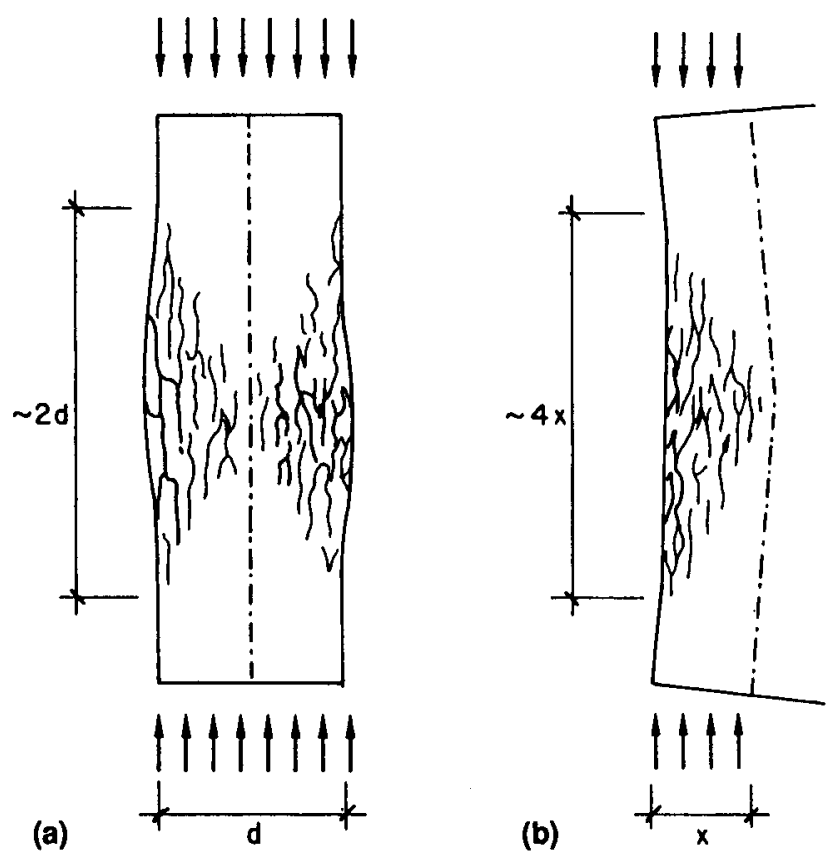

Bild 3.8: Lokalisierung der Verformungen im druckbeanspruchten Beton

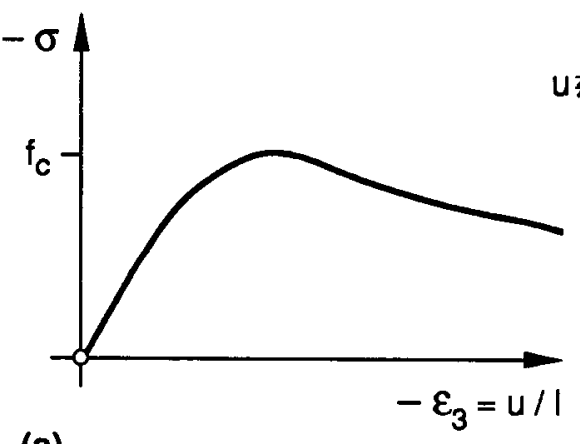

(a)

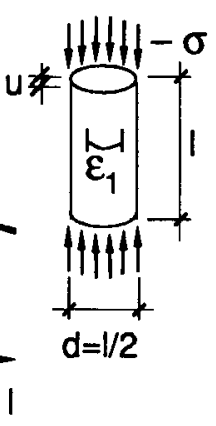

(b)

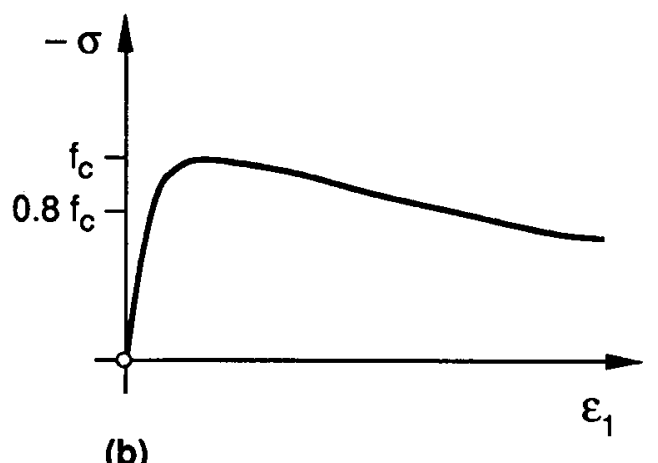

(b)

Bild 3.9: Spannung als Funktion der mittleren Lăngsstauchung und der Querdehnung (Prüfkörper mit $\ell / d=2$ )

Für $0 \leq|\sigma| \leq 0.8 f_{c}$ ist das Verhatten etwa linear und die Mikrorisse haben keinen grossen Einfluss auf die Verformungscharakteristik. Im Bereich $0.8 f_{c} \leq|\sigma| \leq f_{c}$ pflanzen sich die Risse in die Zementmatrix fort und der Beton spaltet sich in Lamellen auf, was sich durch die grosse Zunahme der Querdehnungen erkennen lăsst. Nach dem Erreichen der Druckfestigkeit $f_{c}$ erfolgt die Entfestigung; auch diese Phase ist durch grosse Querdehnungen gekennzeichnet. 
Bild 3.10 zeigt die Spannungs-Dehnungs-Diagramme (mittlere Stauchung im Entfestigungsbereich) für verschiedene Betonfestigkeiten. Diese Kurven sind aus der Arbeit von Wang, Shah und Naaman (1978) entnommen. Es ist ersichtlich, dass die wăhrend des Bruchvorganges dissipierte Energie praktisch unabhăngig von der Betonfestigkeit ist. Je höher die Festigkeit ist, desto grösser ist die bis zum Bruch gespeicherte Energie; damit resultiert bei hohen Betonfestigkeiten eine rasche Entfestigung.

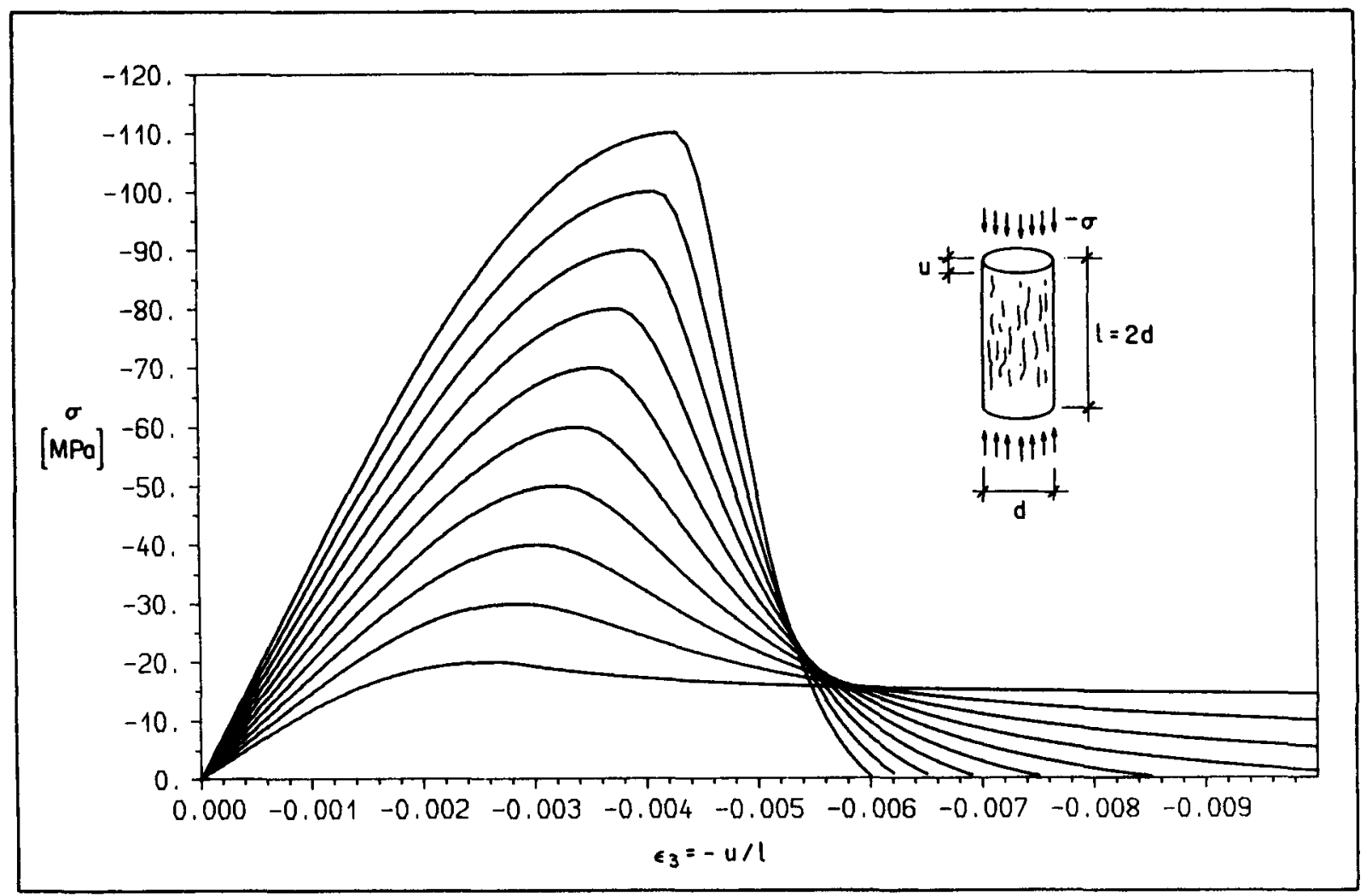

Bild 3.10: Spannungs-Dehnungs-Diagramme des Entfestigungsbereichs für verschiedene Betonfestigkei$\operatorname{ten}\left(f_{c}=20,30, \ldots 110 \mathrm{MPa}\right)$

Bei lăngeren Probekörpern wird wăhrend der Belastung mehr Energie gespeichert, so dass ein spröderes Verhalten entsteht. Im Bild 3.11 sind die Last-Verformungs-Charakteristiken für verschiedene Probekörperlăngen dargestellt. Der Berechnung dieser Kurven liegt die Annahme zugrunde, dass die Entlastung ausserhalb des Entfestigungsbereichs mit dem Tangentenmodul für $\sigma=0$ erfolgt. Für $t_{c}=$ $30 \mathrm{MPa}$ tritt eine Entfestigungsinstabilităt bei $\ell / \ell_{\mathrm{s}} \approx 4$ ein, für $\mathrm{f}_{\mathrm{c}}=50 \mathrm{MPa}$ bei $\ell / \ell_{\mathrm{s}} \approx 2$.

Diese Resultate sind im Bild 3.11d graphisch zusammengestellt. Der Entfestigungsstabilitätsfaktor für Probekörper mit einer Schlankheit $\ell / d=1$ ist als Funktion der Betonfestigkeit ${ }_{C} c$ dargestellt. Die Last-Verformungs-Beziehung im Entfestigungsbereich ist nicht linear, so dass der Entfestigungsstabilitătsfaktor spannungsabhăngig ist. Der dargestellte Wert ist der obere Grenzwert und kann aus dem Spannungs-Dehnungs-Diagramm wie folgt hergeleitet werden:

$$
\frac{x}{l / d}=\frac{d}{l} \cdot \frac{1}{1-\frac{\left.\frac{d \sigma}{d \varepsilon}\right|_{\varepsilon}=0}{(d \sigma / d \varepsilon)_{\text {mi n }}}}
$$




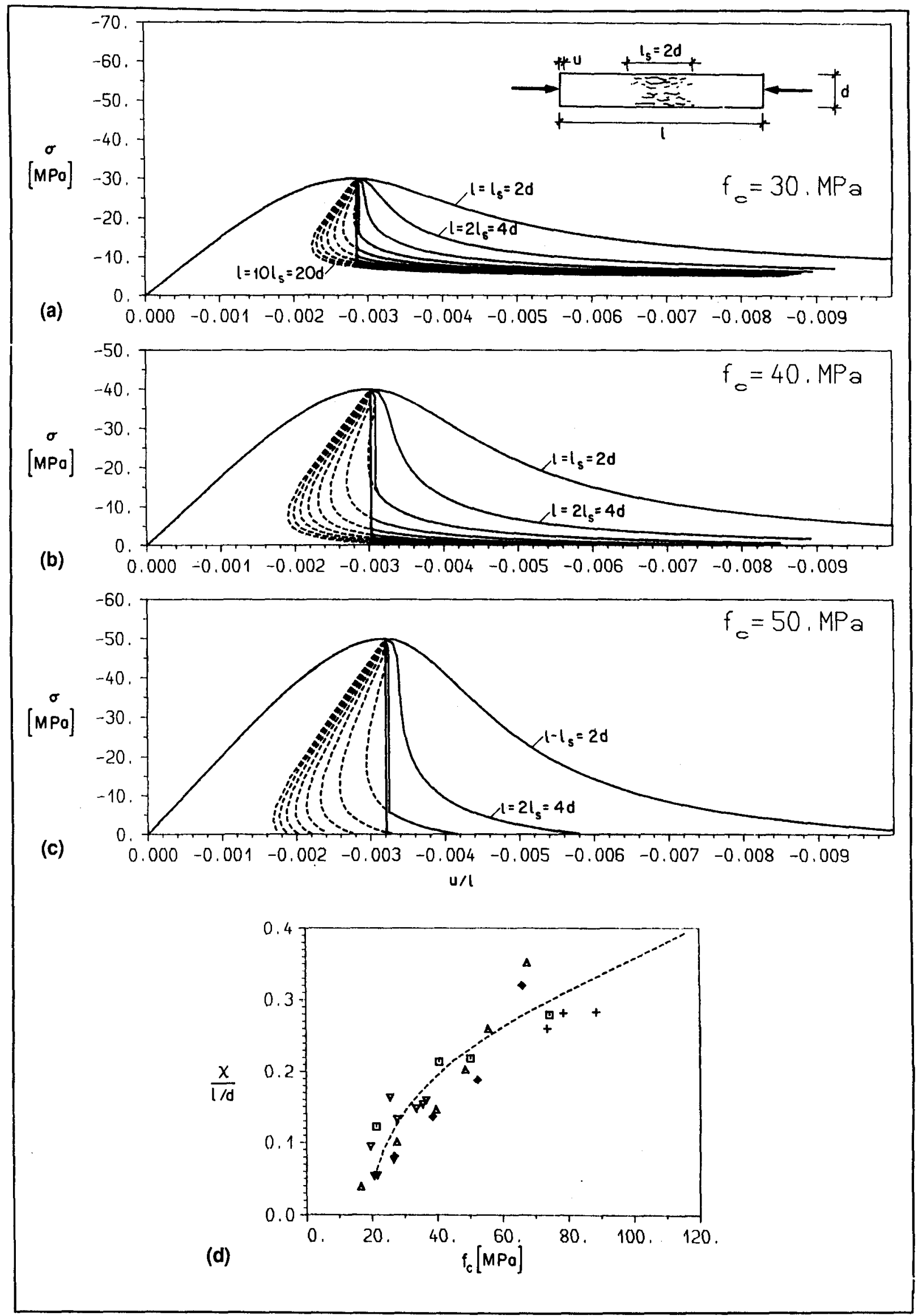

Bild 3.11: (a), (b) und (c) Spannungs-Dehnungs-Diagramme für verschiedene Probekơrperlăngen $\left(\ell / \ell_{s}=1,2, \ldots 10{\text { mit } \ell_{s}}=2 d\right)$; (d) Entfestigungsstabilitătsfaktor. 
Die Beziehung, die aus dem analytischen Ansatz (Bild 3.10) gewonnen werden kann (gestrichelte Linie), zeigt eine gute Uebereinstimmung mit den experimentell ermittelten Werten, die aus der Auswertung der Spannungs-Dehnungs-Beziehungen der folgenden Arbeiten stammen:

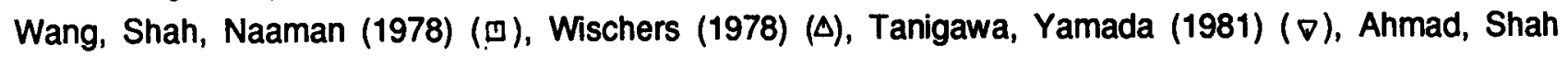
(1982) (x), Ahmad, Shah (1985) ( + .

Das Lăngenverhältnis $\iota / d$, bei dem eine Entfestigungsinstabilităt eintritt, ist mit $\chi=1$ der reziproke Wert der dargestellten Grösse.

Da im Beton durch die Druckbeanspruchung Querzugspannungen auftreten (Bild 3.7), wird die Betonzugfestigkeit infolge einer Druckspannung reduziert. Diese Interaktion ist im Bild 3.12a festgehalten. Die Resultate der von Kupfer (1973) durchgeführten Experimente sind in Form einer Bruchfigur ("Fliessfigur") dargestellt.

Beim Erreichen der durch die Bruchfigur definierten Spannungskombination (Punkt A im Bild 3.12b) entsteht ein Trennriss verbunden mit einer raschen Entfestigung analog wie bei der reinen Zugbeanspruchug. Die Zugspannung fällt ab, bis der Probekörper in zwei Teile getrennt wird (Punkt B). Diese sind jedoch noch nicht in feine Lamellen aufgespalten, so dass die Druckbeanspruchung bis zur Druckfestigkeit erhöht werden kann (Punkt C).

Wenn eine Querbewehrung vorhanden ist, wird die Zugbeanspruchung bei der Rissbildung von der Bewehrung aufgenommen. Bei einer Erhöhung der Zugkraft bilden sich weitere Risse aus, so dass ein ăhnlicher Zustand entsteht wie beim einachsig druckbeanspruchten Beton im Entfestigungsbereich. Dies bedeutet, dass in diesem Fall mit einer effektiven Festigkeit $f_{c e} z u$ rechnen ist, die kleiner als $\mathrm{f}_{\mathrm{c}}$ ist.

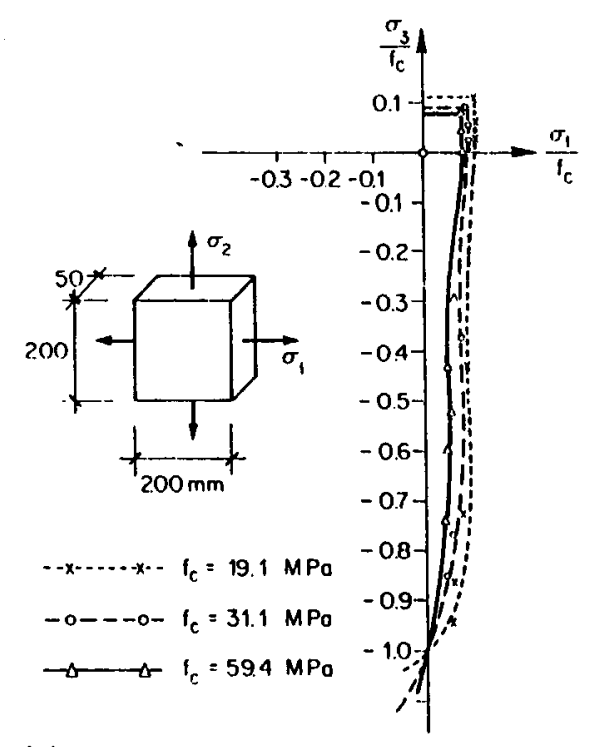

(a)

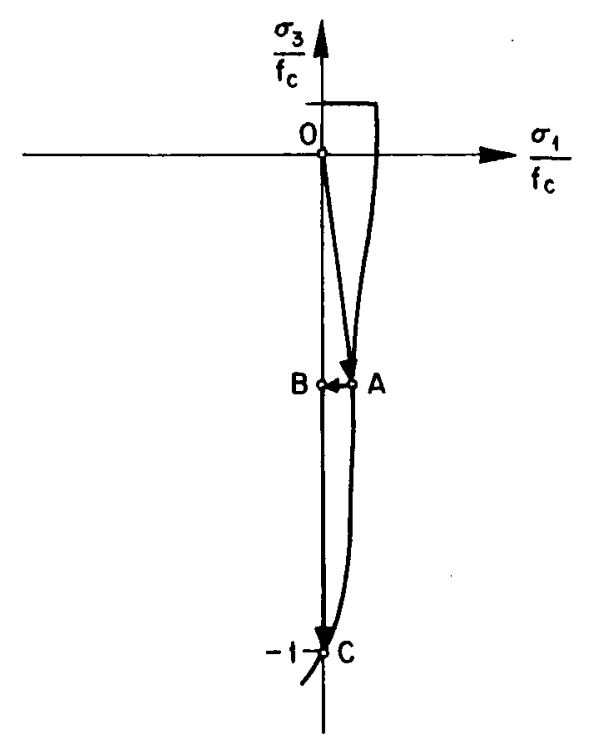

(b)

Bild 3.12: Bruchbedingung für Beton im Druck-Zug-Bereich: (a) Versuche von Kupfer (1973); (b) möglicher Lastweg. 
(a)
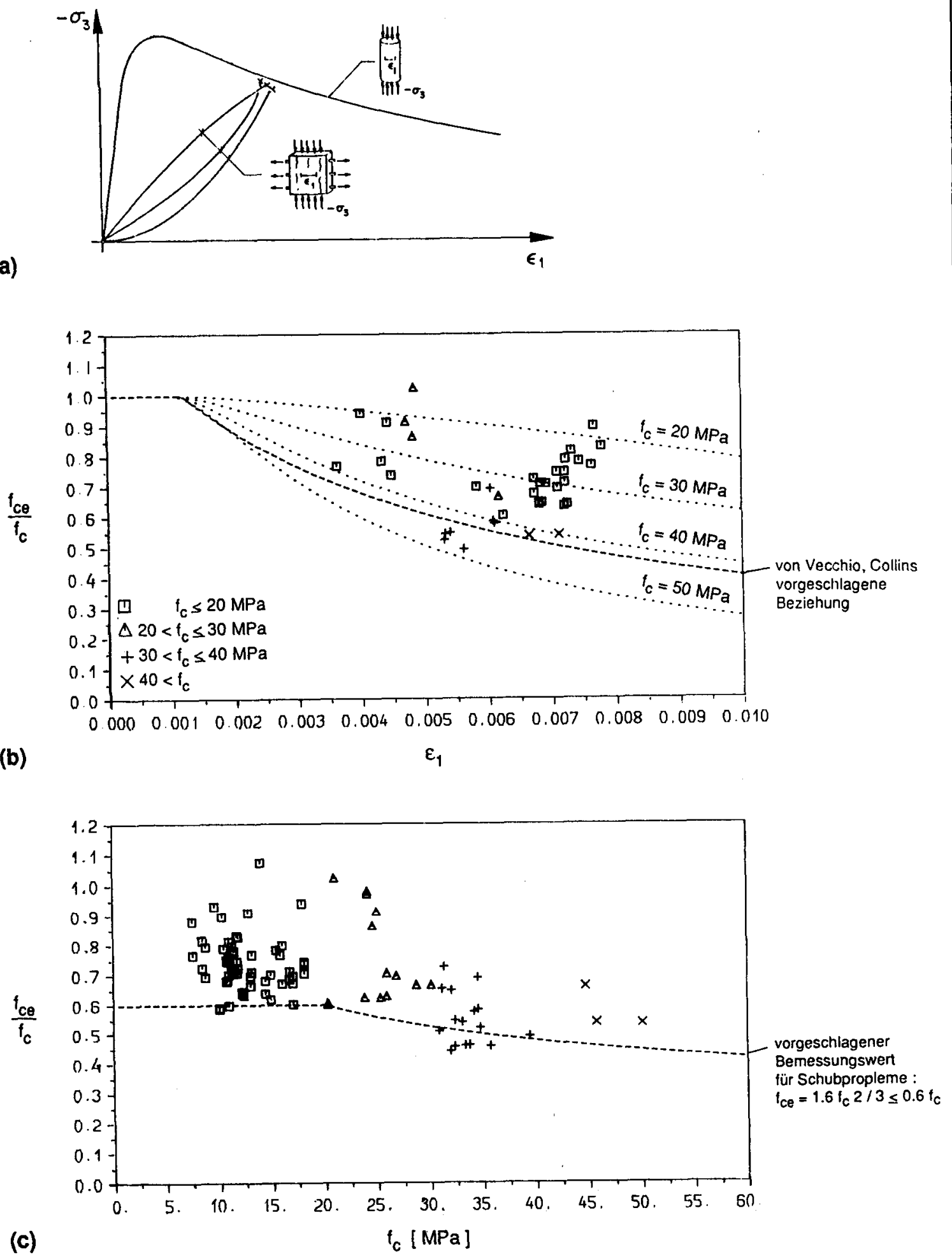

Bild 3.13: Verhalten bei reinem Druck und Druck mit aufgezwungener Querdehnung: (a) qualitativer Vergleich; (b) quantitativer Vergleich; (c) effektive Festigkeit bei Schubproblemen. 
Bild 3.13a zeigt die Druckspannung als Funktion der Querdehnung einer Stahlbetonscheibe mit DruckZug-Beanspruchung verglichen mit dem Verhalten eines einachsigen Druckversuchs. Wenn die Querdehnung als Mass für die Entfestigung betrachtet wird, kann die effektive Betonfestigkeit einer Stahlbetonscheibe aus diesem Vergleich ermittelt werden.

Diese Analogie wird mit den im Bild 3.13b dargestellten Versuchsresultaten bestătigt. Die Punkte im Diagramm beschreiben die effektive Festigkeit im Steg von schubbeanspruchten Stahlbetonbalken als Funktion der Querdehnung im Steg. Für die Auswertung der Versuchsresultate und die Berechnung der dargestellten Grössen sei auf Anhang A1 (Tabelle A1.6) hingewiesen. Die punktierten Kurven stellen die Druckspannung als Funktion der Querdehnung von einachsigen Druckversuchen dar. Die von Ahmad, Shah (1982) angegebenen Spannungs-Dehnungs-Kurven tür zwei Betonsorten sind extrapoliert worden. Dass die Querdehnung eine Abnahme der effektiven Festigkeit verursacht, haben auch Vecchio, Collins (1982, 1986) festgestelt. Die von diesen Autoren vorgeschlagene Beziehung zeigt eine gute Uebereinstimmung mit den Versuchsresultaten (gestrichelte Linie im Bild 3.13b).

Die Versuchsresultate zeigen den Einfluss einer weiteren wichtigen Grosse. Wie schon beim einachsigen Druckversuch beschrieben, ist die Entfestigung vor allem bei Beton mit hoher Festigkeit ausgeprăgt. Dasselbe zeigen hier die $\sigma_{3}-\varepsilon_{1}$-Kurven einachsiger Druckversuche und die Resultate von Schubversuchen. Im Bild $3.13 \mathrm{c}$ ist das Verhăltnis $\mathrm{f}_{\mathrm{ce}} \mathrm{ff}_{\mathrm{c}}$ der ausgewerteten Schubversuche (Tabellen A1.5 und A1.6) in Funktion der Zylinderfestigkeit $t_{c}$ dargestellt. Es ist ersichtlich, dass als Bemessungsgrösse

$$
f_{c e}=0.6 \cdot f_{c} \quad \text { für } f_{c} \leq f_{c, 0}=20 \mathrm{MPa}
$$

und

$$
f_{c e}=0.6 \cdot f_{c, 0}\left(\frac{f_{c}}{c, 0}\right)^{2 / 3} \quad \text { für } \quad f_{c} f_{c, 0}=20 \mathrm{MPa}
$$

angenommen werden kann.

Da bei Schubproblemen die Querdehnung im allgemeinen in einem relativ kleinen Bereich schwankt, kann auf die Berücksichtigung des Einflusses der Querdehnung auf die effektive Festigkeit verzichtet werden. Mit kleinen Werten von $f_{c e} / f_{c}$ ist eventuell im Bereich von grossen plastischen Verformungen zu rechnen (plastische Gelenke mit grossen Rotationen). Günstige Werte der effektiven Festigkeit kőnnen hingegen mit Vorspannung der Lăngs- und Schubbewehrungen erzielt werden.

Die infolge Druckbeanspruchung vorhandene Querzugspannung (Bild 3.7) kann reduziert oder sogar zum Verschwinden gebracht werden, wenn gleichzeitig eine Querdruckbeanspruchung aufgebracht wird. Die damit erreichte Erhöhung der Festigkeit kann mit der Hypothese von Mohr-Coulomb beschrieben werden. Das Bild 3.14a zeigt die Fliessbedingung (Bruchbedingung) und die von Menne (1977) zusammengestellten Versuchsergebnisse. Für nicht zu grosse Querdruckpressungen ist die Uebereinstimmung der Experimente mit der Fliessbedingung

$$
\sigma_{3} \geq-f_{c}+4 \cdot \sigma_{1}
$$

gut. 

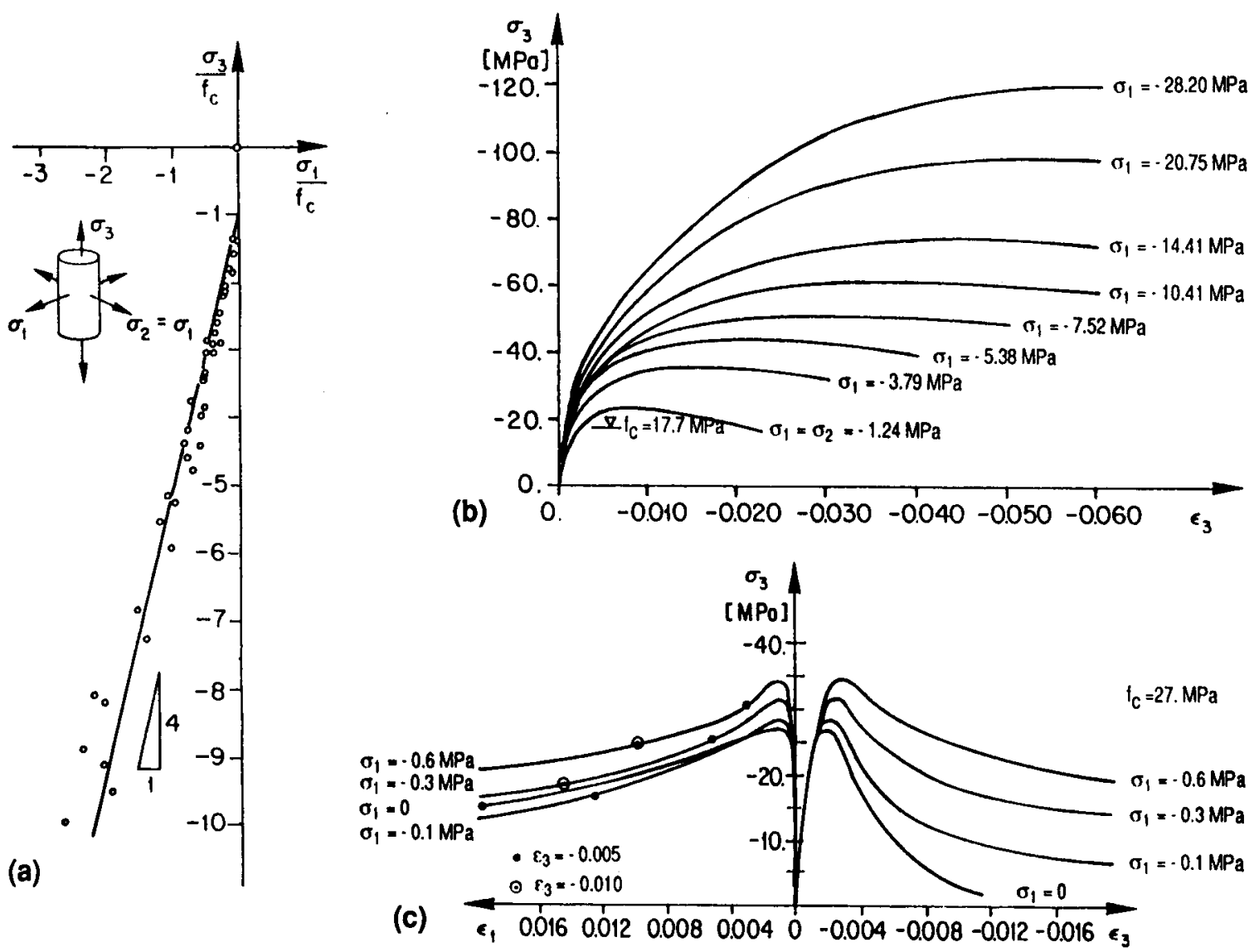

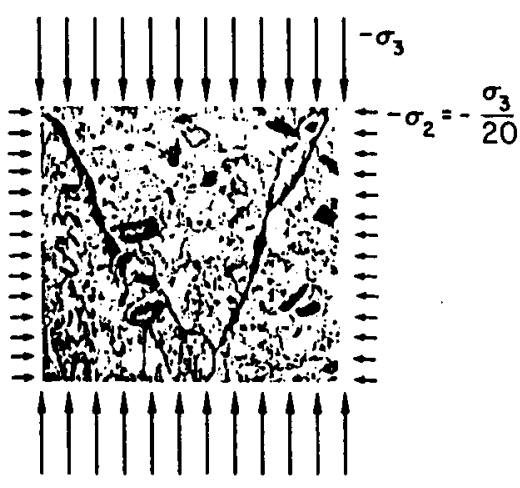

(d)
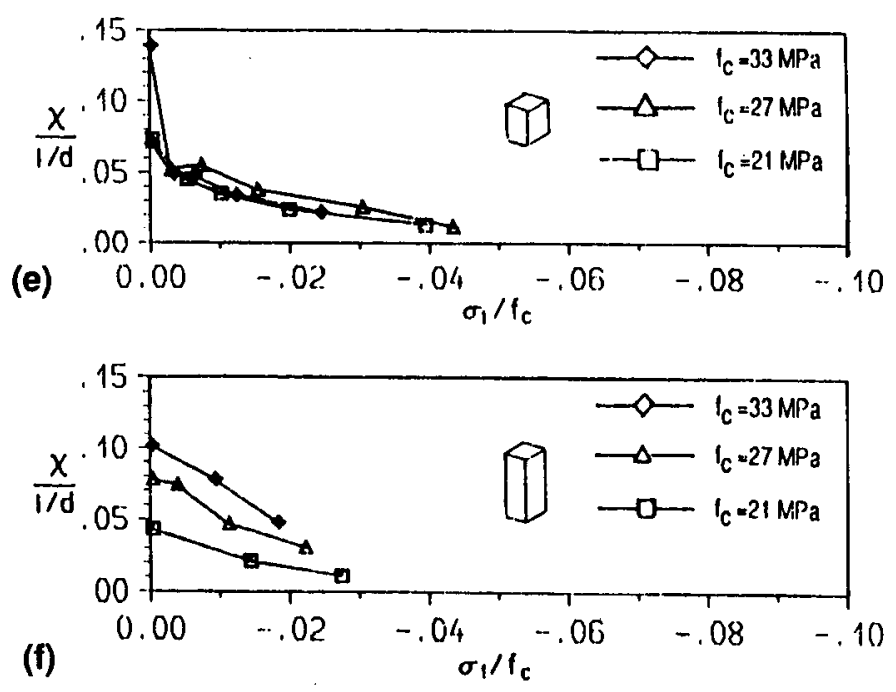

Bild 3.14: Verhalten bei dreiachsiger Druckbeanspruchung:

(a) Fliessbedingung und Versuchsresultate aus Menne (1973); (b) Spannungs-DehnungsBeziehung aus Richart, Brandtzaeg, Brown (1928); (c) aus Kosaka, Tanigawa, Hatanaka (1985); (d) Bruchbild); (e) Entfestigungsstabilitătsfaktor ermittelt bei Wüffelversuchen; ( $f$ ) Entfestigungsstabilitătsfaktor ermittelt bei Prismenversuchen. 
In den Bildern 3.14b und 3.14c sind die Spannungs-Dehnungs-Kurven der von Richart, Brandtzaeg und Brown (1928) sowie Kosaka, Tanigawa, Hatanaka (1985) durchgeführten Versuche dargestellt. Aus diesen Bildern ist ersichtlich, dass aus Querdruck nicht nur eine Erhohung der Festigkeit, sondern auch der Duktilităt resultiert. Dieses Phănomen lăsst sich mit den unterschiedlichen Bruchvorgăngen erklären. Wie Kanellopoulos (1986) beschrieben hat, wird im Fall von genügend grossen Querpressungen der Bruch primăr durch Schiebung entlang einer geneigten Gleitflăche eingeleitet und nicht wie beim einachsigen Druckversuch vorwiegend durch das Instabilwerden der längsgespaltenen Struktur. Ein solches Bruchbild zeigt Bild 3.14d, das der Arbeit von van Mier (1984) entnommen ist. Die mit der Querpressung zunehmende Duktilităt geht auch aus den Bildern 3.14e und 3.14f hervor. Der Entfestigungsstabilitătsfaktor ist als Funktion der Querpressung für drei Betonsorten dargestellt. Die Werte wurden durch Auswertung von in den Arbeiten von Kosaka, Tanigawa und Hatanaka $(1984,1985)$ enthaltenen Spannungs-Dehnungs-Kurven von Prismenversuchen (Bild 3.14e) und Würfelversuchen (Bild 3.14f) ermittelt. Wie den beiden Bildern zu entnehmen ist, erfolgt eine grosse Abnahme des Entfestigungsstabilitătsfaktors bereits bei kleinen Querpressungen.

Dreiachsige Druckbeanspruchungen entstehen dann, wenn eine aussere Querpressung wirkt, oder wenn die Querdehnung durch umgebende Betonmassen oder durch Bewehnung behindert wird. Solche Fâlle und im speziellen die Wirkung einer Umschnürungsbewehrung in einer Stütze werden im Kapitel 4 năher behandelt.

Breite Risse schwächen die Betonstruktur. Wenn sich der Riss wieder schliesst, oder wenn eine Schiebung der getrennten Betonkörper entlang des Risses erfolgt, kann wegen der Reibung und der Unebenheit der Rissufer (sogenannte Rissverzahnung) wiederum eine Druckkraft senkrecht zum Riss oder schrăg dazu übertragen werden. Bei kleinen Rissőffnungen u und genügend grossen Schiebungen $\checkmark$ wirkt die Mikroverzahnung auf der gesamten Rissfläche (Bild 3.15b). Bei grossen Rissőffnungen hingegen kann die Kraft nur noch in lokalen Zonen (Makroverzahnung) übertragen werden (Bild 3.15a). Bild 3.15c zeigt die Druckspannung $\sigma_{c}$, die mit der Mikroverzahnung übertragen werden kann, als Funktion der Rissöffinung $u$ und der Riss-Schiebung v. Es ist zu beachten, dass diese Kraftübertragung von der Verformungsgeschichte abhăngig ist. Die dargestellte Beziehung stammt aus einer Auswertung der von Walraven (1981) durchgeführten Versuche. Bei konstanter Rissőffnung wurde in diesen Experimenten die Riss-Schiebung stetig erhöht. Bei wiederholten Beanspruchungen kőnnen die Rissufer durch lokale Brüche allmăhlich zerstört werden, so dass der Widerstand gegen eine weitere Verschiebung entsprechend abnimmt. Bei fehlendem Kontakt zwischen den Rissufem erfolgt auch keine Kraftübertragung. Im Bild 3.15d sind die Rissoffnung u und die Riss-Schiebung $v$, mit denen die beiden Betonkörper in Kontakt bleiben, dargesteltt. 


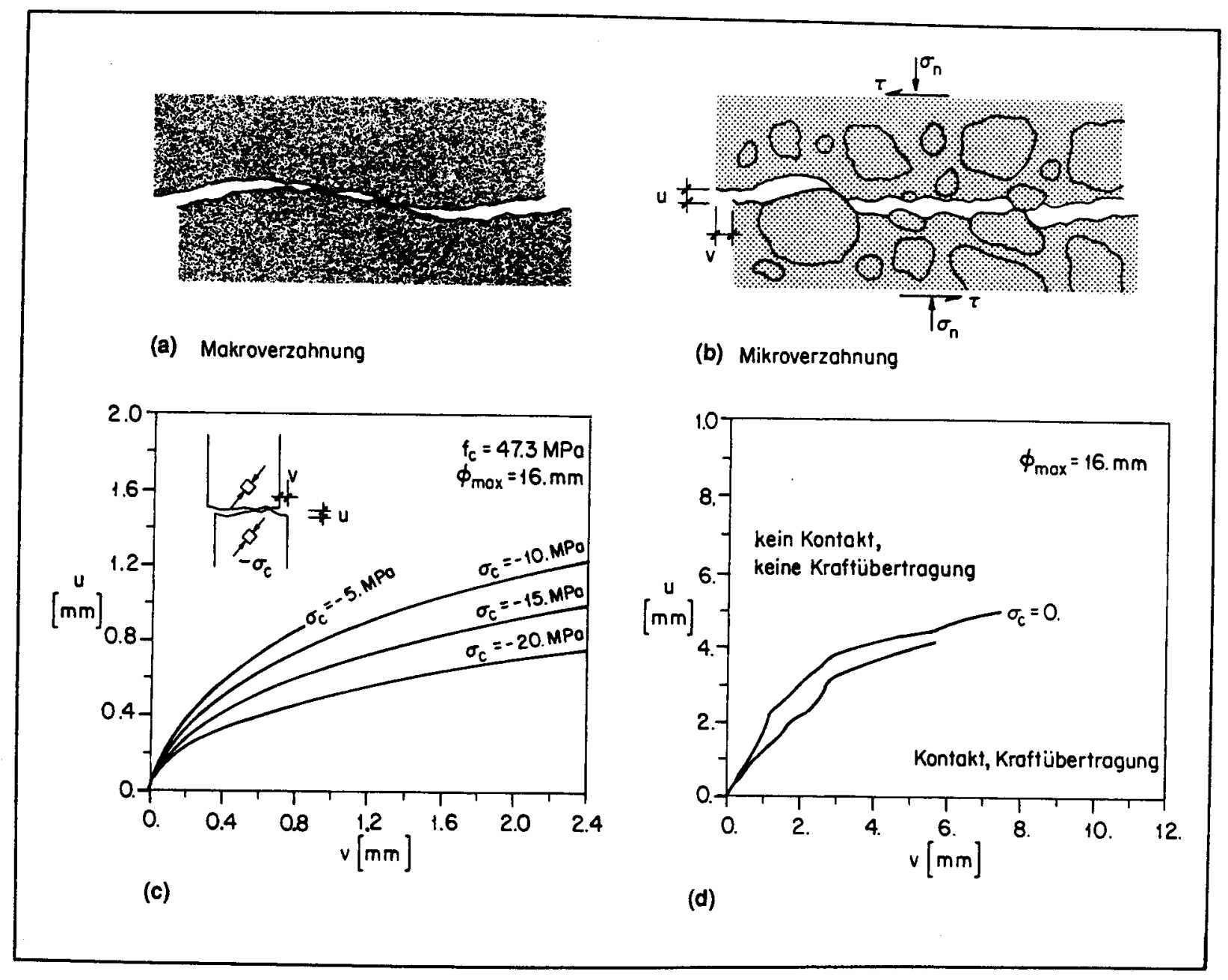

Bild 3.15: (a) Makroverzahnung; (b) Mikroverzahnung; (c), (d) Kraftübertragung als Funktion der Rissöffnung und der Riss-Schiebung. 


\section{Verhalten von Stahlbeton}

\subsection{Zug}

Das Verhalten eines zugbeanspruchten Stahlbetonelementes ist im Bild 4.1a dargestellt (Versuchsresultate von Jaccoud, Francou und Camara (1984)). Die ungerissene Phase weist ein beinahe lineares Verhalten auf. Die im Bild 3.6a erkennbare Nichtlinearităt des zugbeanspruchten Betons hat im allgemeinen keinen grossen Einfluss auf die Charakteristik von Stahlbetonstäben, da diese plastische Verformung in einem sehr kleinen Bereich lokalisiert ist. Der erste Riss entsteht mit der Normalkraft

$$
N=\left(A_{c}-A_{s}\right) \cdot f_{c t}+A_{s} \cdot n \cdot f_{c t}=A_{c} \cdot f_{c t} \cdot(1+\rho(n-1)) \times A_{c} \cdot f_{c t}
$$

mit

$\mathrm{n}$ = Verhăltnis Elastizitătsmodul Stahl/Elastizitătsmodul Beton

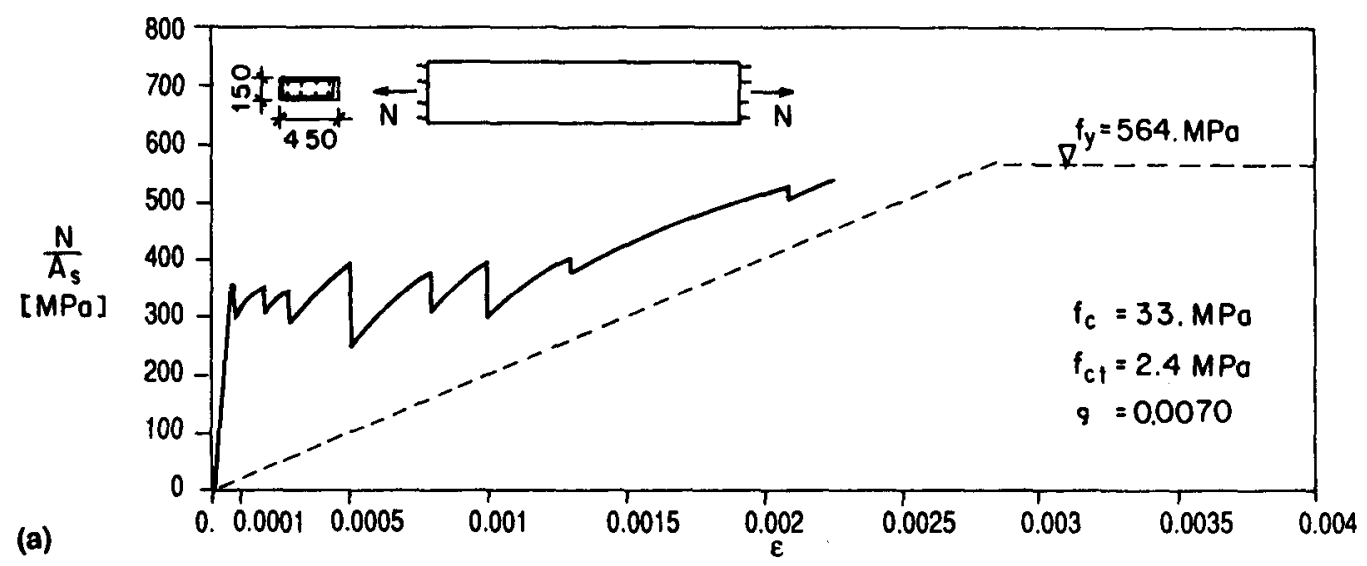

(b)

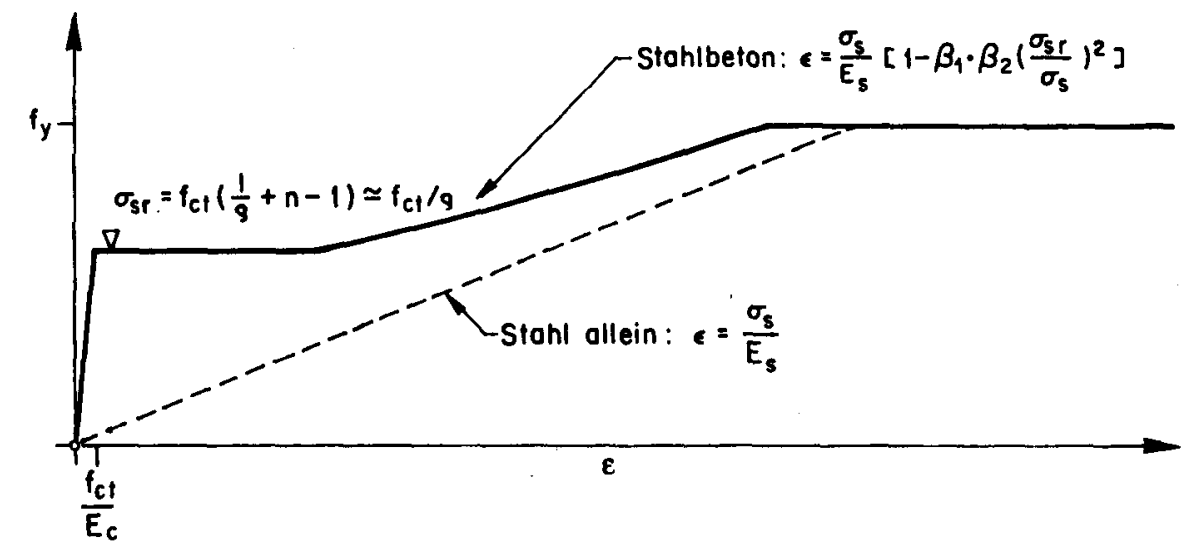

Bild 4.1: Last-Verformungs-Charakteristik eines zugbeanspruchten Stahlbetonstabes; (a) Versuchsergebnisse von Jaccoud, Francou und Camara (1984); (b) Analytische Beziehung nach CEB-FIP Code Modèle (1978). 
Ist die Messbasis grösser als die im Abschnitt 3.2 definierte kritische Lănge von $400 \mathrm{~mm}$, tritt eine Entfestigungsinstabilităt ein. Die Normalkraft făllt abrupt ab, bis die Bewehrung aktiviert wird. Wenn die Bewehrung genügend stark ist, kann die Last wieder erhöht werden, bis ein zweiter Riss entsteht. Dieser Vorgang kann wiederholt werden, bis der Rissabstand so klein geworden ist, dass über den Verbund die erforderliche Kraft zur Bildung eines neuen Risses nicht mehr eingeleitet werden kann. Wird der Stahlbetonstab weiter gedehnt, verbreitert sich die Oeffnung der vorhandenen Risse. In dieser Phase nehmen auch die Stahlspannungen und somit die Normalkraft wieder zu, bis die Streckgrenze erreicht wird. Dieses Verhalten kann analytisch mit der empirisch ermittelten Beziehung des CEB-FIP Code modèle (1978) vereinfachend beschrieben werden. Sie ist mit den zugehörigen Gleichungen im Bild 4.1b dargesteltt.

Das horizontale Plateau entspricht der Phase der Rissbildung. Bei einer weiteren Erhöhung der Kraft wird der Zugbeanspruchungseffekt im Beton zwischen den Rissen (tension stiffening) mit einem hyperbolischen Ansatz berücksichtigt. Die Einflüsse von Verbundgüte und Beanspruchungsart werden mit den Parametern $\beta_{1}$ und $\beta_{2}$ berücksichtigt:

$\begin{array}{lll}\beta_{1} & =1.0 & \text { für Bewehrungsstäbe mit Rippen } \\ \beta_{1} & =0.5 & \text { für glatte Bewehrungsstäbe } \\ \beta_{2} & =1.0 & \text { für die erste Belastung } \\ \beta_{2} & =0.5 & \text { für Dauerlasten oder zyklische Belastung }\end{array}$

Wenn die totale Fliesskraft der Bewehrung kleiner als die Risslast ist, kann die Last vor der Verfestigung des Stahls nicht mehr erhöht werden; es bildet sich somit nur ein einziger, sehr breiter Riss. Diese Lokalisierung der Verformungen ist vor allem im Hinblick auf die Gebrauchstauglichkeit zu vermeiden. Die Bilder $4.2 \mathrm{a}$ und $4.2 \mathrm{~b}$ zeigen ein solches Verhalten für Bewehrungsgehalte $\rho=0.0045$ und $\rho=0.0060$ (Versuchsergebnisse von Jaccoud, Francou und Camara (1984), Messbasis $=1400 \mathrm{~mm}$ ). Mit solchen Bewehrungsgehalten entsteht auch bei kleinen mittleren Dehnungen ein breiter Riss.

Eine derartige Lokalisierung der Verformungen kann auch die Duktilităt negativ beeinflussen, wenn die Zugfestigkeit der Bewehrung kleiner ist als die Risslast. Dieses Verhalten ist im Bild 4.2c qualitativ dargestellt. Die maximal mögliche Verlăngerung des Zugstabes ist in einem solchen Fall durch die Länge der Zone im Rissbereich, wo der Verbund gelöst ist und sich die Bewehrung verformen kann, von der Dehnung beim Erreichen der Zugfestigkeit und von der Verlängenung der Bewehrung im Entfestigungsbereich (Bild 3.1b) gegeben. Eine gute Verbundqualităt verursacht somit bei derart schwach bewehrten Stahlbetonstäben einen Sprödbruch. 


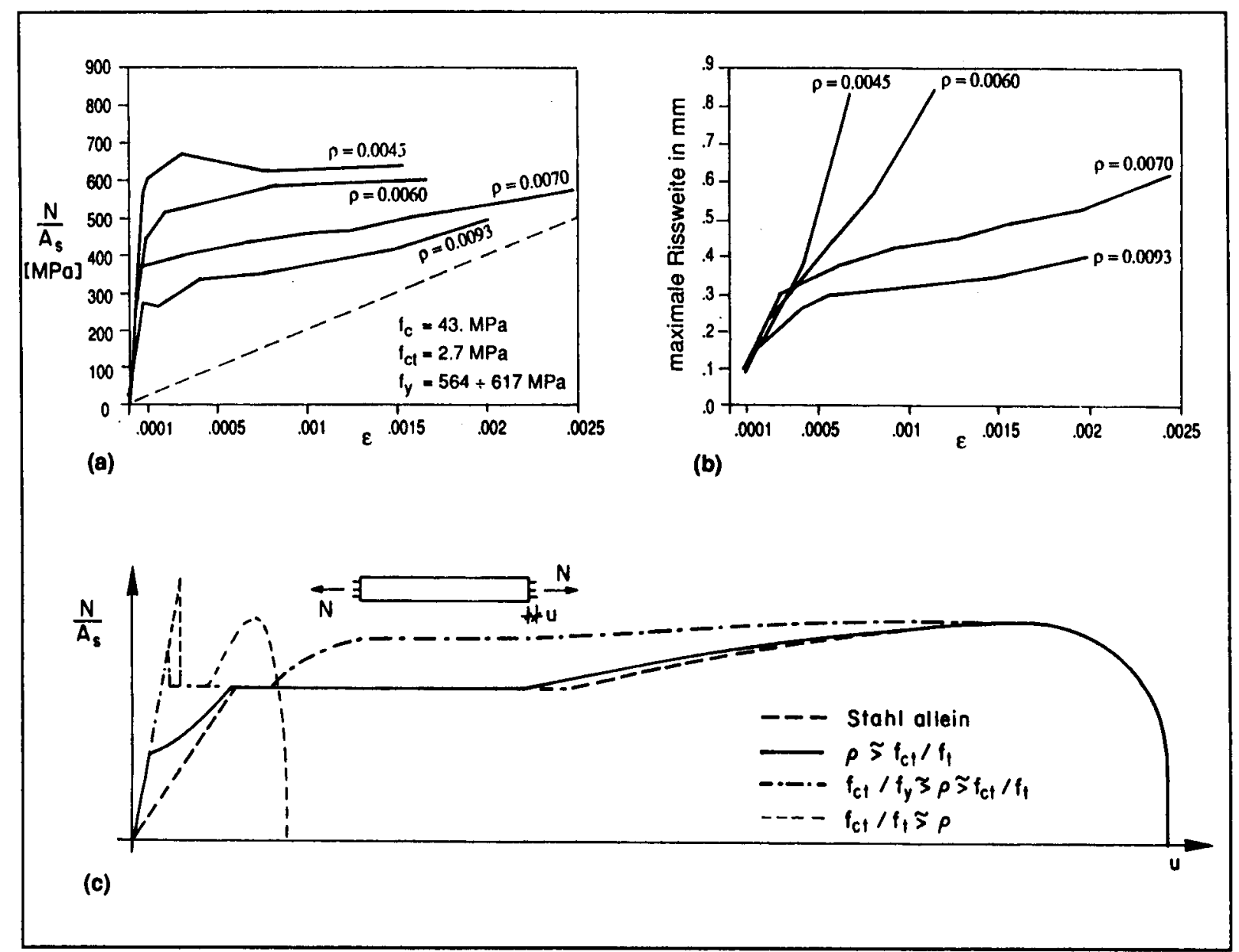

Bild 4.2: Last-Verformungs-Charakteristik als Funktion des Bewehrungsgehaltes; (a) und (b) experimentell ermitteltes Verhalten (Jaccoud, Francou und Camara (1984); (c) qualitatives Verhalten bei Lokalisienung der Verformung. 


\subsection{Druck}

Das Verhalten unbewehrter Druckglieder mit einachsiger oder dreiachsiger Druckbeanspruchung wurde im Abschnitt 3.2 erlăutert. Die üblichenweise vorhandenen Quer- und Lăngsbewehrungen beeinflusssen das Tragverhalten wie folgt:

\section{- Querbewehrung:}

Sie wirkt als Umschnürung. Der daraus resultierende dreiachsige Spannungszustand erhöht die Festigkeit und die Duktilităt des Betons. Sie wirkt zudem als Sicherung gegen das Knicken der Lăngsbewehrung.

\section{- Långsbewehrung:}

Sie nimmt direkt einen Teil der Druckkraft auf und kann die konzentrierten Umlenkkrăfte der Umschnürbewehrung auf den Beton verteilen.

Bewehrte Stützen sind somit durch die dreiachsige Betonbeanspruchung infolge Umschnürung und die direkte Druckkraftaufnahme der Lăngsbewehrung gekennzeichnet. Die beiden Effekte werden getrennt behandelt.

Die Zugbeanspruchung in der Umschnürungsbewehrung wird durch die Querdehnung des druckbeanspruchten Betons aktiviert. Bild 4.3a zeigt einen Ausschnitt der im Bild 3.14c dargestellten Spannungs-Dehnungs-Beziehungen von dreiachsig beanspruchtem Beton sowie die Stahlspannungen, die mit den zugehörigen Qeurdehnungen aktiviert werden. Die Fliessgrenze der Umschnürungsbewehrung wird erreicht, wenn sich der Beton bereits in der Entfestigungsphase befindet; für gewöhnliche Bewehrungsstăhle mit $f_{y} \simeq 500 \mathrm{MPa}$ ist die Abnahme der Festigkeit jedoch nicht gross. Dies gilt jedoch nur für den umschnürten Bereich, da sich die Betonüberdeckung schon abgelöst hat. Stăhle mit hőherer Festigkeit wie z.B. Spannstăhle mit $f_{y} \simeq 1600 \mathrm{MPa}$ müssen hingegen vorgespannt werden, damit sie ohne allzu grosse Reduktion der Betonfestigkeit voll aktiviert werden.

Bei kreisrunden Druckgliedern erfolgt die Umlenkung der Bewehrungskraft konstant verteilt über den Umfang. Zwischen den Bügeln bleibt aber der Zylindermantel unbeansprucht, so dass nicht die ganze umschnürte Zone dreiachsig beansprucht ist. Dies ist aus dem von Kanellopoulos (1986) vorgeschlagenen Spannungsfeld klar ersichtlich (Bild 4.3b). Gemäss diesem Modell ist die massgebende dreiachsig beanspruchte Zone nur der innere Zylinder mit dem Durchmesser $d-s$ ( $s=$ Bügelabstand). Die dort vorhandene Querpressung betrăgt

$$
\sigma_{1}=-\frac{\omega_{t} \cdot f}{1-\frac{\pi}{4} \cdot \frac{s}{d}} \quad \text { mit } a_{t}=\frac{2 \cdot A_{s} \cdot f y}{s \cdot d}
$$

Mit der Zunahme der Festigkeit (siehe Abschnitt 3.2)

$$
\Delta f_{c 3}=-4 \cdot \sigma_{1}
$$


ergibt sich folgende mittlere Festigkeit in dimensionsloser Form:

$$
\frac{f_{c 3}}{f_{c}}=1+4 \cdot q_{t} \cdot \frac{\left(1-\frac{s}{d}\right)^{2}}{1-\frac{\pi}{4} \cdot \frac{s}{d}}
$$

Diese Gleichung ist für verschiedene Werte von s/d im Bild 4.3c ausgewertet. Die Uebereinstimmung mit den eingetragenen Versuchsresultaten von Richart, Brandtzaeg und Brown (1929) mit s/d = 0.1 ist gut. Die Versuchsdaten sind im Anhang A2 zusammengestellt. 


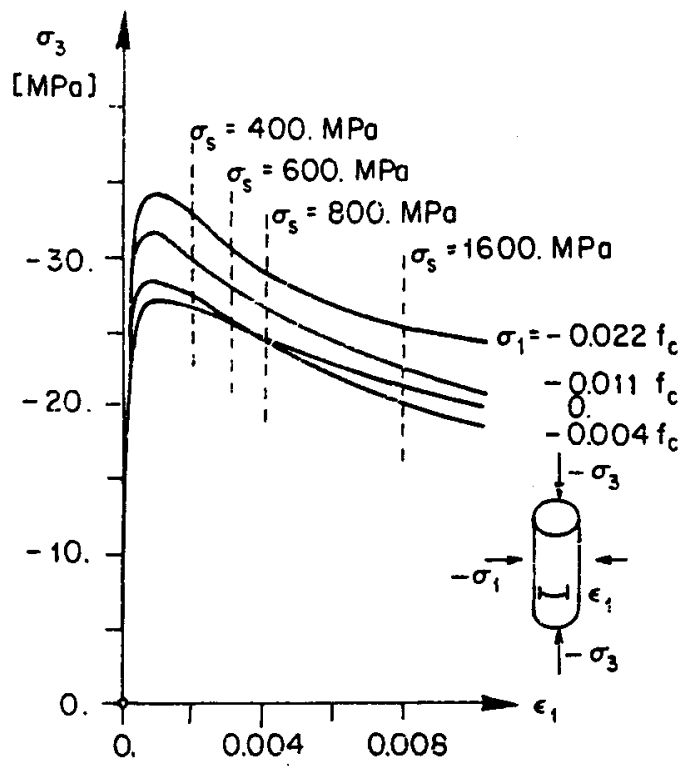

(a)
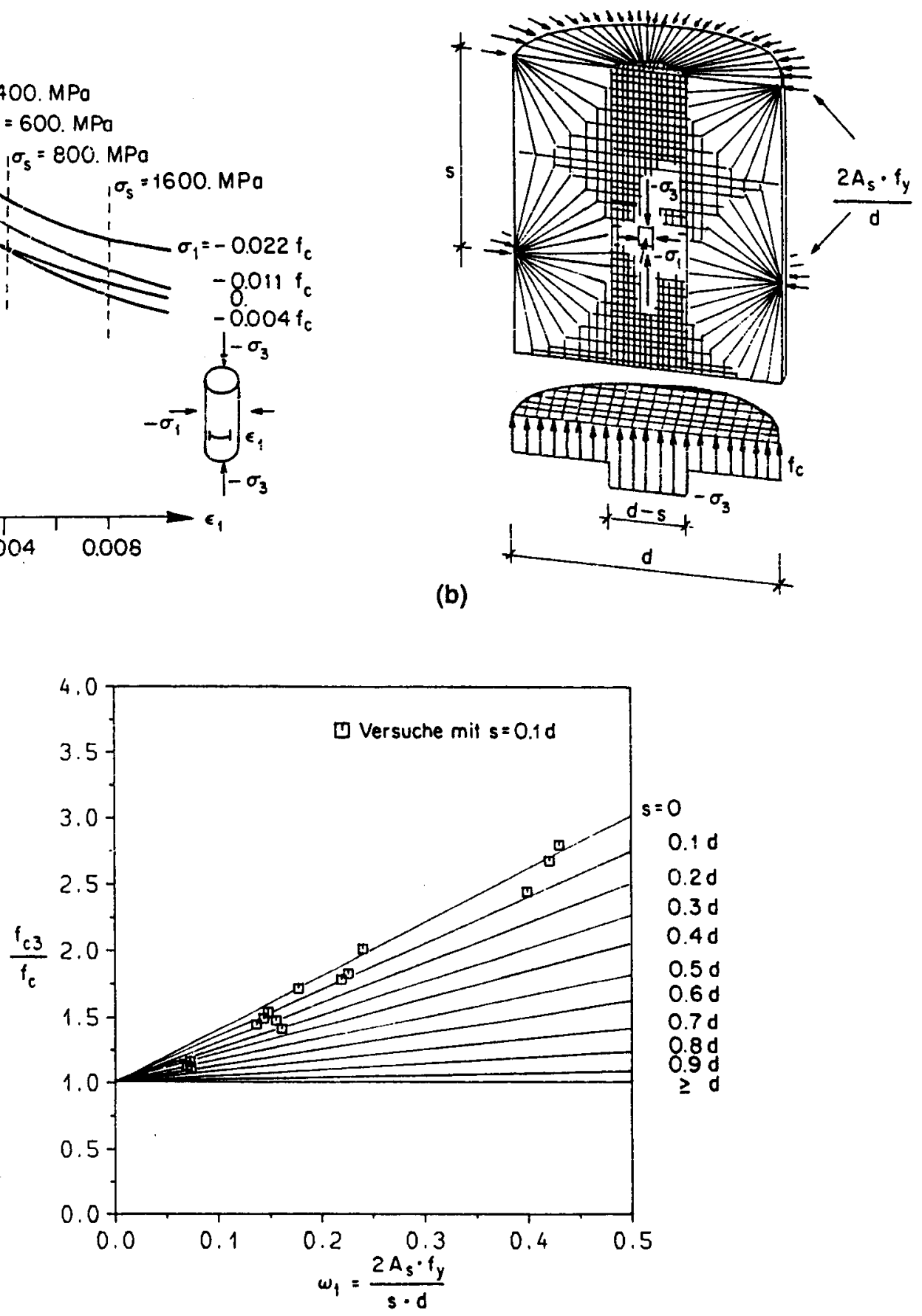

(c)

Bild 4.3: (a) Druckspannung als Funktion der Querdehnung (s. auch Bild 3.14c) und aktivierte Spannung in der Querbewehrung von kreisnunden Stützen; (b) Spannungsfeld nach Kanellopoulos (1986) für die Beschreibung der Wirkung der Umschnürbewehrung; (c) mittlere Festigkeit als Funktion des mechanischen Querbewehrungsgehaltes für $s / d=0,0.1$ bis 1 , und Versuchsresultate von Richart, Brandtzaeg, Brown (1929). 


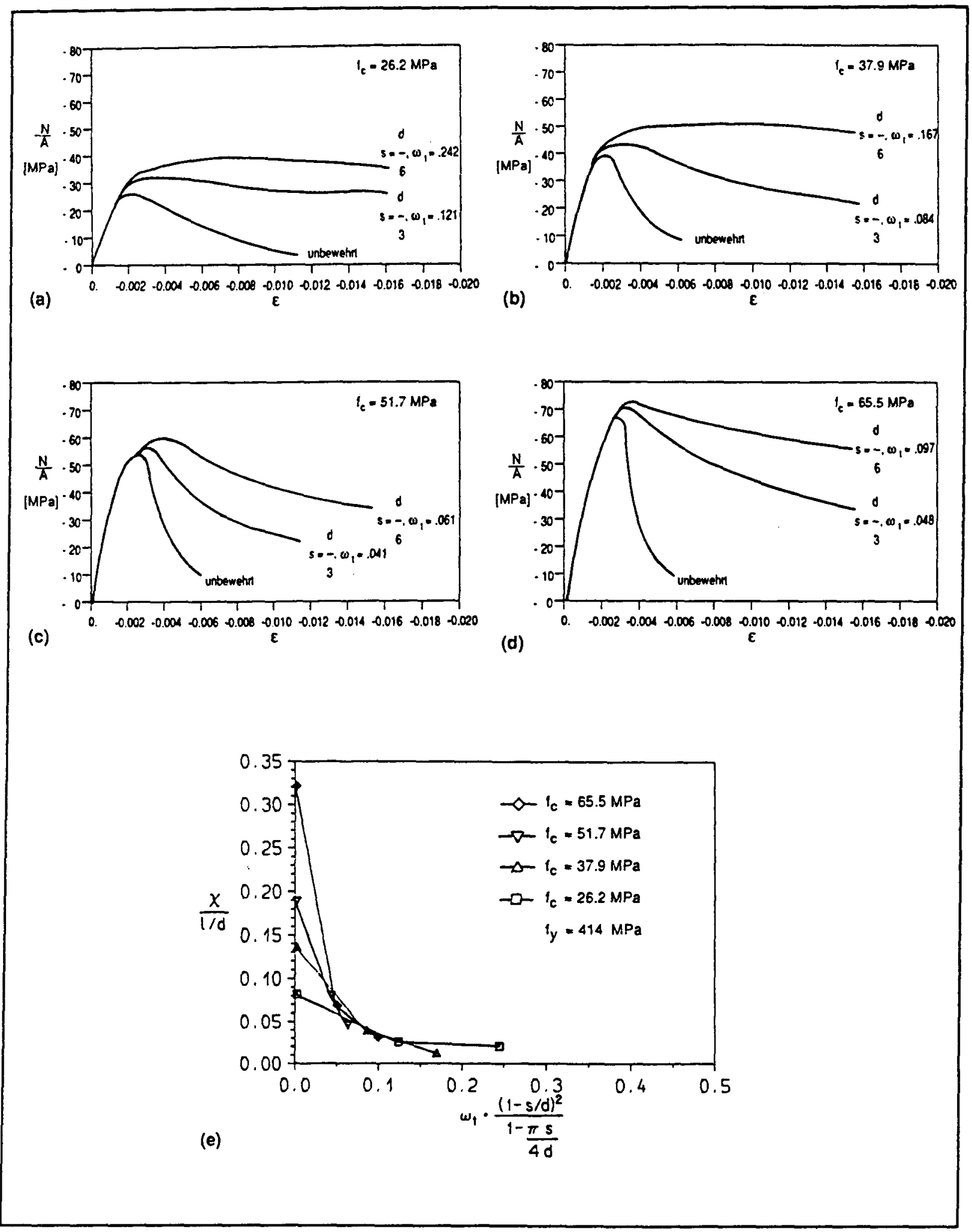

Bild 4.4: Versuche an zylindrischen umschnūrten Probekörpern von Ahmad und Shah (1982): (a) - (d) Spannungs-Dehnungs-Beziehungen; (e) Entfestigungsstabilitătsfaktor. 
Die Făhigkeit der Lăngsbewehrung, die Umlenkkrăfte der Umschnürungsbewehrung auf den Beton zu verteilen, wird oft überschătzt. Diese Wirkung wird erst für grosse Stabdurchmesser und kleine Bügelabstănde wichtig.

Die Bilder 4.4a bis $4.4 \mathrm{~d}$ illustrieren die Spannungs-Dehnungs-Beziehungen (mittlere Dehnung im Verformungsbereich $\ell=2 \cdot d)$ der von Ahmad und Shah (1982) durchgeführten Versuche an umschnürten zylindrischen Probekörpern. Es ist ersichtlich, dass die Umschnürungsbewehrung die Duktilität betrăchtlich erhöht. Dies ist im Bild 4.4e, in welchem der maximale Entfestigungsstabilitätsfaktor als Funktion des effektiven Querbewehrungsgehaltes für verschiedene Betonfestigkeiten dargestellt ist, zusammenfassend ausgewertet. Für eine Festigkeit von $65.5 \mathrm{MPa}$ kann zum Beispiel der Faktor $\chi \cdot \mathrm{d} / \ell=0.32$ (Entfestigungsinstabilităt für $\ell / \mathrm{d}>3.0$ ) mit einer relativ kleinen Querbewehrung ( $q$ $=0.097$ ) auf 0.03 (instabil für $l / d>33$ ) reduziert werden. Eine ähnliche Feststellung wurde bereits aufgrund der Bilder $3.14 \mathrm{e}$ und $3.14 \mathrm{f}$ (dreiachsige Druckbeanspruchung) gemacht.

Bei quadratischen Druckgliedern mit einfachen Bügeln oder quadratischen Spiralen als Umschnürungsbewehrung enfolgt die Umlenkung der Bewehrungskraft nur in den Eckbereichen. Diese bewirkt eine Verkleinerung der dreiachsig beanspruchten Zone. Die aus der Arbeit von Kanellopoulos (1986) stammenden Bilder 4.5a und 4.5b zeigen die Spannungsfelder der umschnürten Zone im Fall einer diskreten Krafteinleitung (a) und einer verteilten Umlenkung (b), die nur bei einem sehr engen Bügelabstand erfolgt. Die Kraftausbreitung in vertikaler Richtung wird erst für Bügelabstănde $s \geq d$ massgebend. Die Grösse der dreiachsig beanspruchten Zone ist somit für $s \leq d$ vom Bügelabstand unabhăngig. Die Grösse dieser Zone kann aus der Auswertung von Versuchsergebnissen empirisch ermittelt werden. Bild 4.5c zeigt die Resultate von Somes (1970). Eine gute Uebereinstimmung mit diesen Werten bringt für $\mathrm{s} / \mathrm{d} \leq 1$ die Beziehung

$$
\frac{f_{c 3}}{f_{c}}=1+2 \cdot q \quad \text { mit } \quad q=\frac{2 \cdot A_{s} \cdot f y}{s \cdot d}
$$

Dies bedeutet, dass die effektiv umschnürte Zone nur die Hălfte der Gesamtflăche ausmacht.

Die Querbewehrung bewirkt auch bei quadratischen Druckgliedern eine Erhőhung der Duktilität. Dies zeigen die Last-Verformungs-Kunen von Bild 4.5d und der zugehörige Entfestigungsstabilitätsfaktor, der im Bild $4.5 e$ ausgewertet ist. 


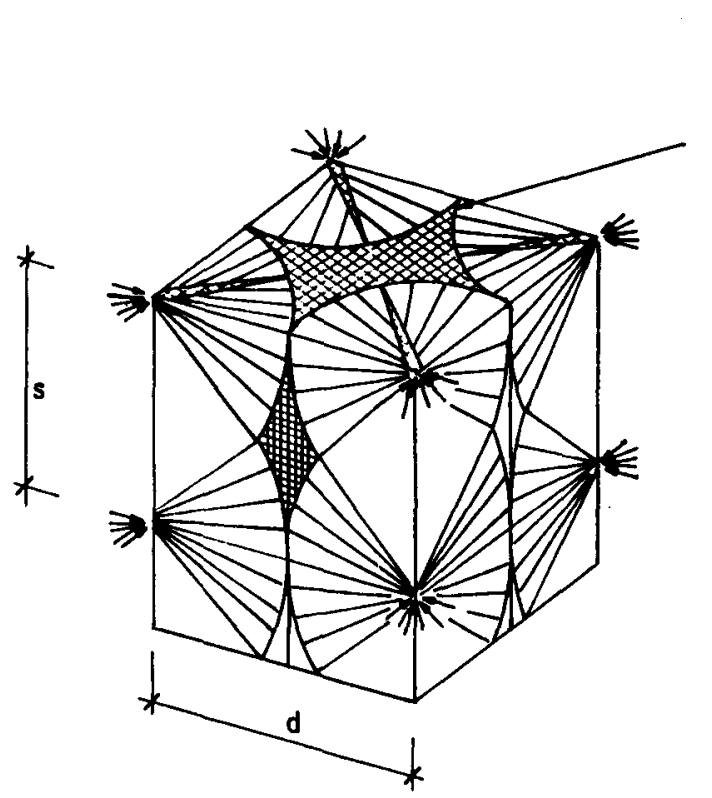

(a)

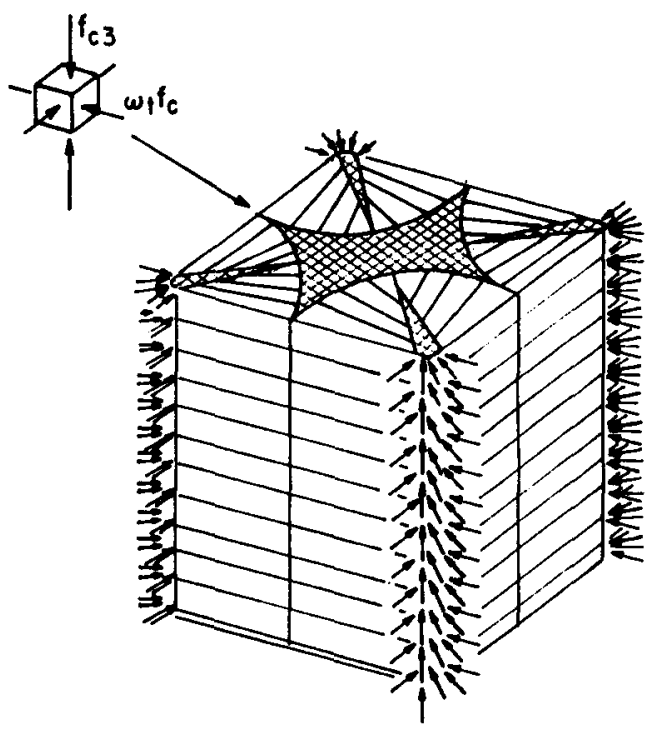

(b)
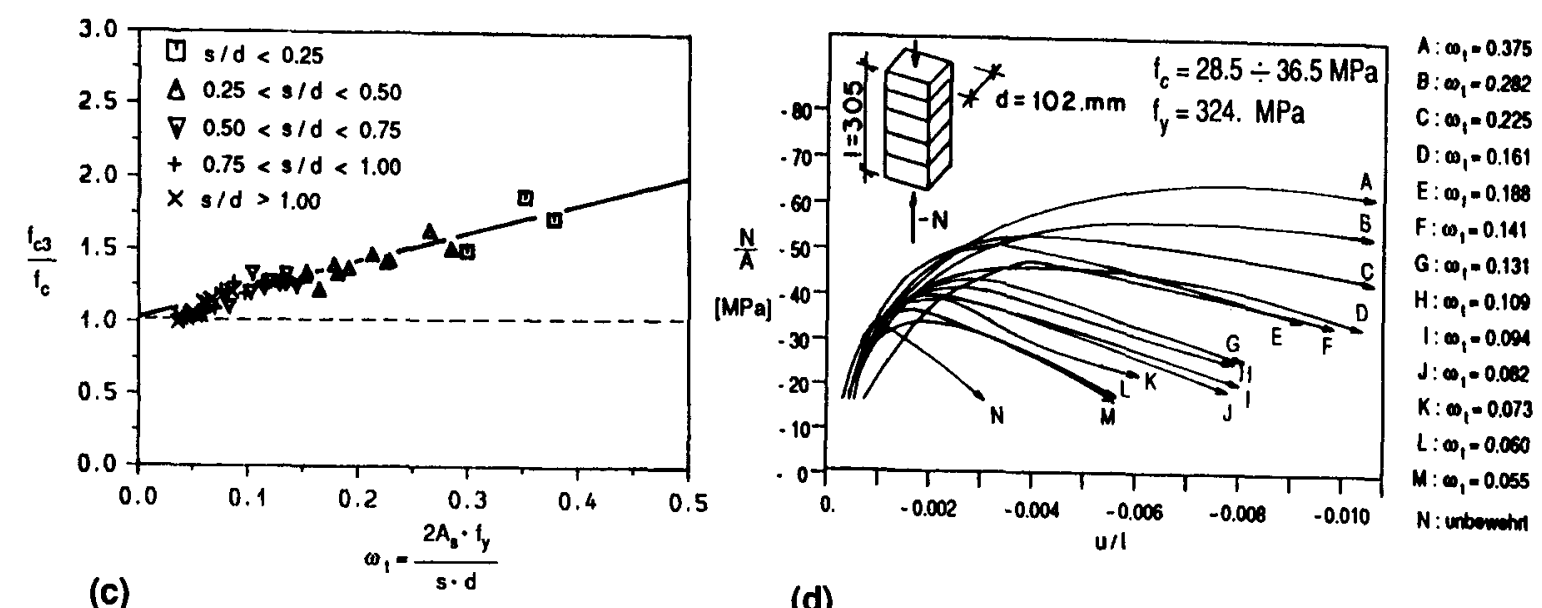

(d)

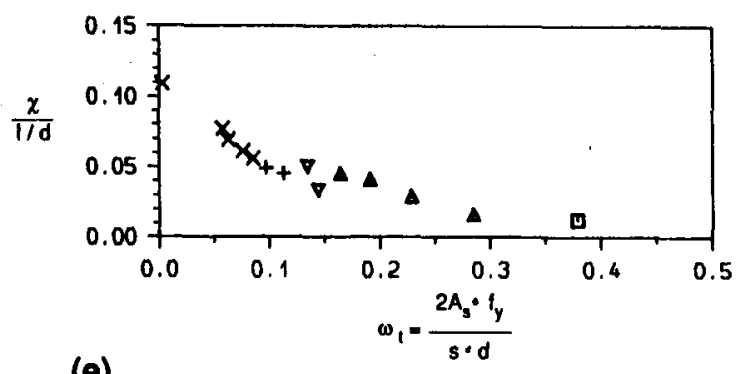

(e)

Bild 4.5: Quadratische Stützen mit einfacher Bügelbewehrung: Spannungsfelder der umschnürten Zone bei mittlerem (a) und bei sehr engem (b) Bügelabstand; (c) mittlere Festigkeit als Funktion des Querbewehrungsgehaltes; (d) Last-Verformungskurven; (e) Entfestigungsstabilitătsfaktor (Versuchsresultate von Somes (1970)). 

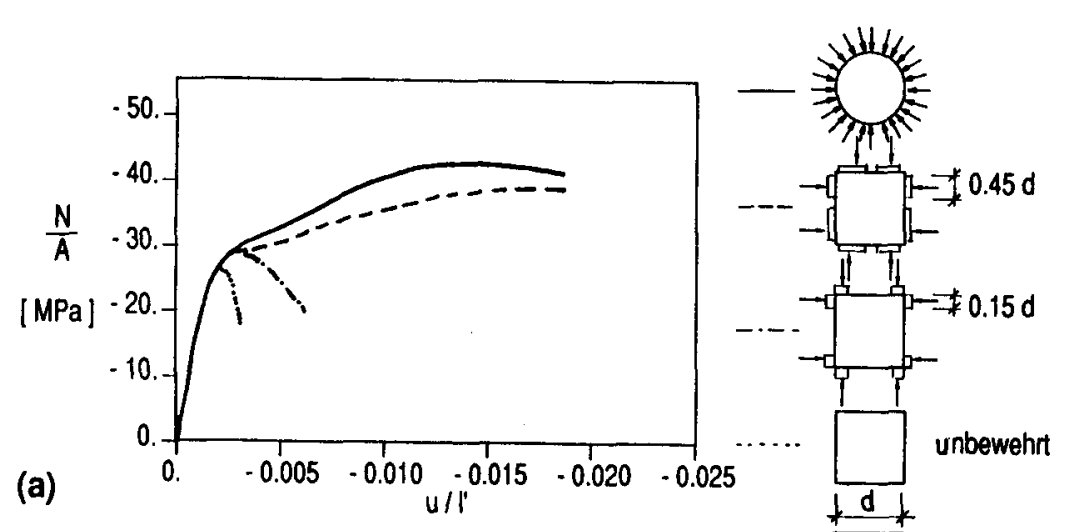

$\mathrm{f}_{\mathrm{c}}=30 . \mathrm{MPa}$

$f_{c}=24 . M P a$

$d=127 . \mathrm{mm}$

$f_{c}=28 \cdot M P a$

Messlänge :

$l^{\prime}=203 . \mathrm{mm}$

$f_{c}=18 . M P a$

$\omega_{1}=0.142$

$\omega_{1}=0.107$

$d=305 . \mathrm{mm}$
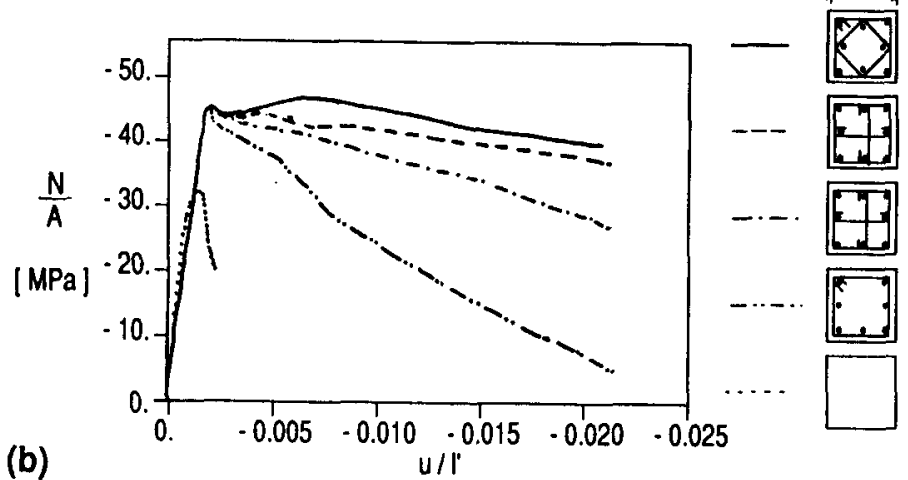

Messlänge

$\omega_{1}=0.107$

$l^{\prime}=381 . \mathrm{mm}$

$\omega_{1}=0.071$

$s / d=0.125$

unbewehrt

$f_{c} \simeq 38 \mathrm{MPa}$

(b)

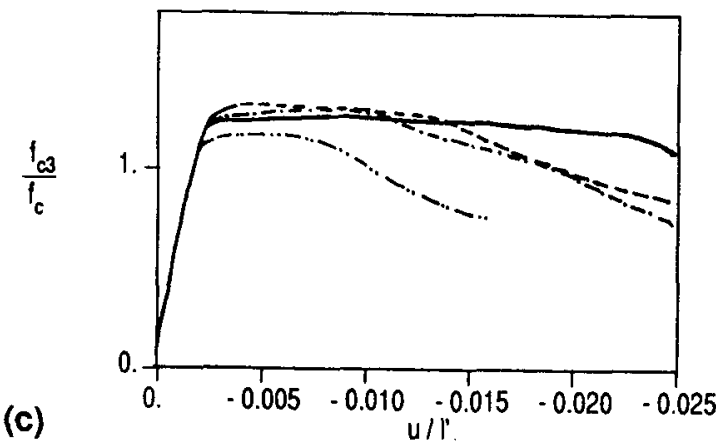

$\omega_{1}=0.071, f_{c}=41 . \mathrm{MPa}, \mathrm{s} / \mathrm{d}=0.083$

एव $\omega_{1}=0.121, \mathrm{l}_{\mathrm{c}}=36 . \mathrm{MPa}, \mathrm{s} / \mathrm{d}=0.094$

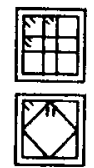

$\omega_{1}=0.096, f_{c}=35 . M P a, s / d=0.125$

$d=305 . \mathrm{mm}$

Messlänge :

$l=102 . \mathrm{mm}$

$\omega_{1}=0.084, f_{c}=41 . \mathrm{MPa}, s / d=0.094$

(c)

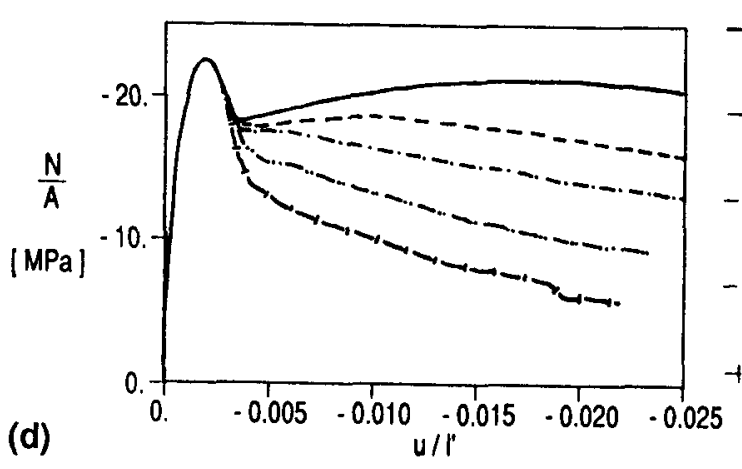

$\omega_{1}=0.220, f_{c}=28.5 \mathrm{MPa}$

弗 $\omega_{1}=0.157, f_{c}=28.5 \mathrm{MPa}$

$d=400 . \mathrm{mm}$

Messlănge :

册 $\omega_{1}=0.126, \mathrm{f}_{\mathrm{c}}=28.6 \mathrm{MPa}$

$l^{\prime}=600 . \mathrm{mm}$

$s / d=0.187$

$\omega_{1}=0.094, f_{c}=28.7 \mathrm{MPa}$

$\mathrm{f}_{\mathrm{y}}=390 \mathrm{MPa}$

$\omega_{t}=0.063, f_{c}=29.0 \mathrm{MPa}$

Bild 4.6: Einfluss der Bügelgeometrie auf das Last-Verformungs-Verhalten: (a) Versuche von Burdette und Hilsdorf (1971); (b) Versuche von Moehle und Cavanagh (1985); (c) Versuche von Sheik und Uzumeri (1980); (d) Versuche von Yashiro, Hanai und Takahashi (1986). 
Dass die Ausbreitung der Umlenkkraft in horizontaler Richtung für die Reduktion der dreiachsig beanspruchten Zone verantwortlich ist, zeigen auch die Versuche von Burdette und Hilsdorf (1971). Prismatische Prüfkörper wurden mit einer externen Bewehrung umschnürt. Bild 4.6a macht deutlich, dass die Grösse der Zone, wo die exteme Querbewehrung umgelenkt wird, die Festigkeit und die Last-Verformungs-Charakteristik stark beeinflusst. Zum Vergleich sind auch die Kurven eines unbewehrten Prüfkörpers und eines Zylinders mit verschmierter Umschnürung eingetragen.

Die Wirksamkeit einer inneren Querbewehnung nimmt zu, wenn die Oberfläche des umschnürten Betons möglichst gleichmăssig mit den Umlenkkråtten der Querbewehnung beansprucht wird. Solche konstruktive Lösungen mit den zugehörigen Last-Verformungs-Kurven sind in den Bildern 4.6b (Versuche von Moehle und Cavanagh, 1985) und 4.6c (Versuche von Sheik und Uzumeri, 1980) dargestellt. Wie Yashiro, Hanai und Takahashi $(1984,1986)$ gezeigt haben, bewirken auch nicht vollverankerte Stäbe eine Erhöhung der Festigkeit und der Duktilităt. Die Querpressung wird in diesem Fall über Verbundspannungen aufgebaut. Bild 4.6d zeigt die Last-Verformungs-Kurven für verschiedene Querbewehrungsgehalte. Der Festigkeitsabfall im Bereich $\varepsilon_{3}=-0.002$ bis -0.003 resultiert aus der Ablösung der Betonüberdeckung, die für diese Stützen relativ gross war.

Die Ablösung der Betonüberdeckung hat ebenfalls auf die Kraftaufnahmefähigkeit der Lăngsbewehrung allgemein einen grossen Einfluss. Nachdem diese Ablosung erfolgt ist, kann das Ausknicken der Lăngsstäbe nur noch von der Querbewehrung verhindert werden. Nimmt man an, die Querdehnung des Kernbetons beeinflusse das Ausknicken der Lăngsstăbe nicht, kann für die Druckbewehrung das LastVerformungs-Diagramm von Bild 3.5 verwendet werden (für naturharte Stăhle). Wie Bild $4.7 a$ zeigt, kann als Einspannlänge $\ell$ der Bügelabstand $s$ angenommen werden. Je nach Schlankheit $s / \Phi_{\ell}$ kann die Last-Verformungs-Charakteristik wie folgt beschrieben werden:

- Für $\mathbf{s} / \Phi_{l}>30$

knickt der Bewehrungsstab bevor die Fliessgrenze erreicht wird; anschliessend erfolgt eine rasche Entfestigung.

- Für $30>s / \Phi_{\ell} \geq 5$

erfolgt das Knicken nachdem Teile des Stabes bis zum Verfestigungsbeginn geflossen sind. Je kleiner die Schlankheit, desto geringer die Entfestigung.

- Für $s / \Phi_{\ell}<5$

kann der Bewehrungsstab bis in den Verfestigungsbereich über die ganze Lảnge gestaucht werden kann.

Die Last-Verformungs-Charakteristik der Stütze kann aus der Superposition der Charakteristiken des Betons und der Längsbewehrung gewonnen werden. Im allgemeinen führt das Ausknicken der Långsbewehrungsstăbe zur Entfestigung der Stütze mit einer Lokalisierung der Verformungen in diesem Bereich. Wenn die Bewehrung hingegen bis in den Verfestigungsbereich gestaucht werden kann, wird mit einem genügend grossen Längsbewehrungsgehalt die Entfestigung des Betons kompensiert. Die Lokalisierung der Verformungen wird damit verhindert, und das Druckglied verhält sich duktil. 
Damit die im Bild 4.7a dargestelte Knickfigur massgebend wird, müssen die Bügel eine ausreichende Steifigkeit autweisen. Bild $4.7 \mathrm{~b}$ zeigt eine Knickfigur, bei der ein Bügel gedehnt wird. Nach Bresler, Gilbert (1961) muss die Bügelsteifigkeit

$$
K \geq \frac{3 \cdot \pi^{4} \cdot E_{l} \cdot l_{\ell}}{4 \cdot s^{3}}=\frac{3 \cdot \pi^{5} \cdot E_{l} \cdot \Phi_{l}^{4}}{256 \cdot s^{3}}
$$

betragen, damit diese Instabilităt nicht eintritt und die Knicklănge weiterhin $\ell_{\mathrm{cr}}=\mathrm{s} / 2$ beträgt. Die Bügelsteifigkeit $K$ ist von der Geometrie abhăngig und für einige typische konstruktive Lösungen in den Bildern $4.7 \mathrm{~d}$ bis $4.7 \mathrm{~g}$ angegeben. Bügel, die vor allem auf Biegung beansprucht werden (Fall Bild 4.7g), können erst mit grossen Stabdurchmessern voll wirksam sein:

$$
\frac{\Phi_{t}}{\Phi_{l}} \geq \frac{\pi}{4} \cdot \sqrt{\frac{E_{l}}{E_{t}} \cdot\left(\frac{d^{\prime}}{s}\right)^{3}}
$$

Für gewöhnliche Verhältnisse $\Phi_{t} / \Phi_{l}$ genügen demnach solche Bügel als Stabilisierungselemente für die Längsstăbe nicht; eine der beiden Lösungen der Bilder 4.7e oder $4.7 f$ ist daher notwendig.

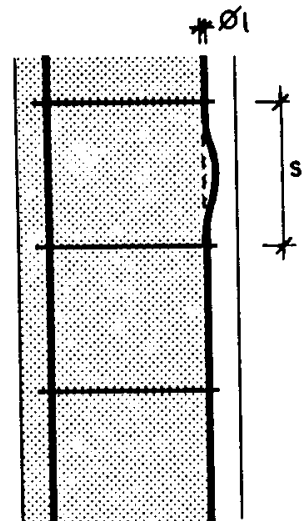

$$
t_{c r}=\frac{s}{2}
$$

(a)

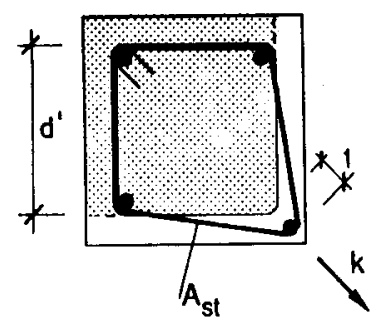

(d)

$K=\frac{A_{s t} \cdot E_{t}}{d^{\prime}}$

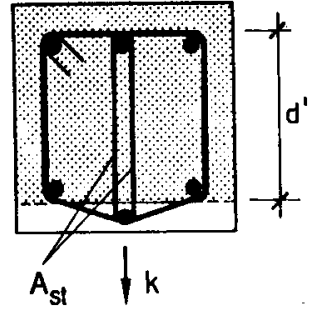

(e)

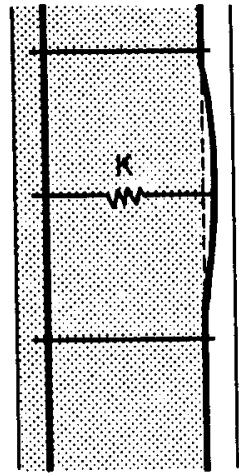

$\frac{s}{2} \leq I_{c r} \leq s$

(b)

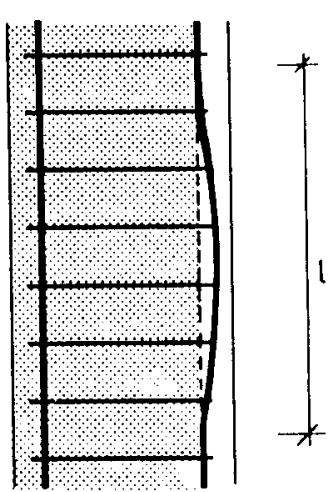

(c)

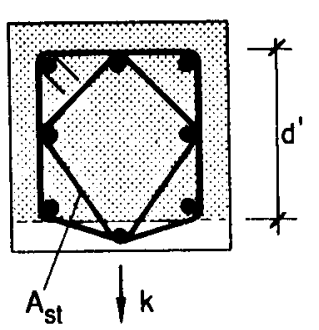

(f)

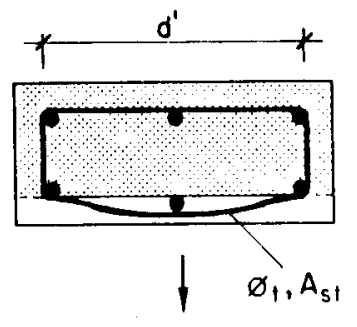

(g)

Bild 4.7: Mögliche Knickfiguren der Lăngsbewehrung und Steifigkeit der Querbewehrung 
Bei eng verbügelten Druckgliedem können beim Ausknicken mehrere Querstäbe aktiviert werden (Bild 4.7c), weshalb die Wirkung der Bügel als konstant verteilt angenommen werden darf. Aus der Lösung der Differentialgleichung

$$
w^{\prime \prime \prime \prime}-N \cdot w^{\prime \prime}+\frac{K}{s} \cdot w=0
$$

für die Auslenkung $w$ mit der Normalkraft des Bewehrungsstabes $N$ und der Bügelsteifigkeit $\mathrm{K} / \mathrm{s}$ resultieren für die dargestellte Knickfigur die kritische Normalkraft

$$
\mathrm{N}_{\mathrm{cr}}=-2 \cdot \sqrt{\frac{7}{3} \cdot \frac{K}{\mathrm{~s}} \cdot \mathrm{E}_{l} \cdot 1_{l}}
$$

und die kritische Spannung

$$
\sigma_{c r}=-\sqrt{\frac{7}{3 \cdot \pi} \cdot \frac{K}{s} \cdot E_{l}}
$$

Es ist zu bemerken, dass diese Spannung vom Stabdurchmesser $\Phi_{\ell}$ unabhăngig ist.

Für die Ermittlung der Bügel- und Lăngsbewehrungssteifigkeiten ist der Tangentenmodul einzusetzen. Beide Bewehrungen können entweder im elastischen, im plastischen oder im Verfestigungsbereich mit den zugehörigen Moduli liegen. Diesen Zusammenhang zeigt Bild 4.8, in dem die Querdehnung $\varepsilon_{t}$ des dreiachsig beanspruchten Betons als Funktion der Längsstauchung $\varepsilon_{\ell}$ und der Querpressung $\sigma_{1}$ dargestellt ist. Im gleichen Bild sind auch die Grenzen zwischen dem elastischen, dem plastischen und dem Verfestigungsbereich für die Lăngs- und die Querbewehrung eingetragen.

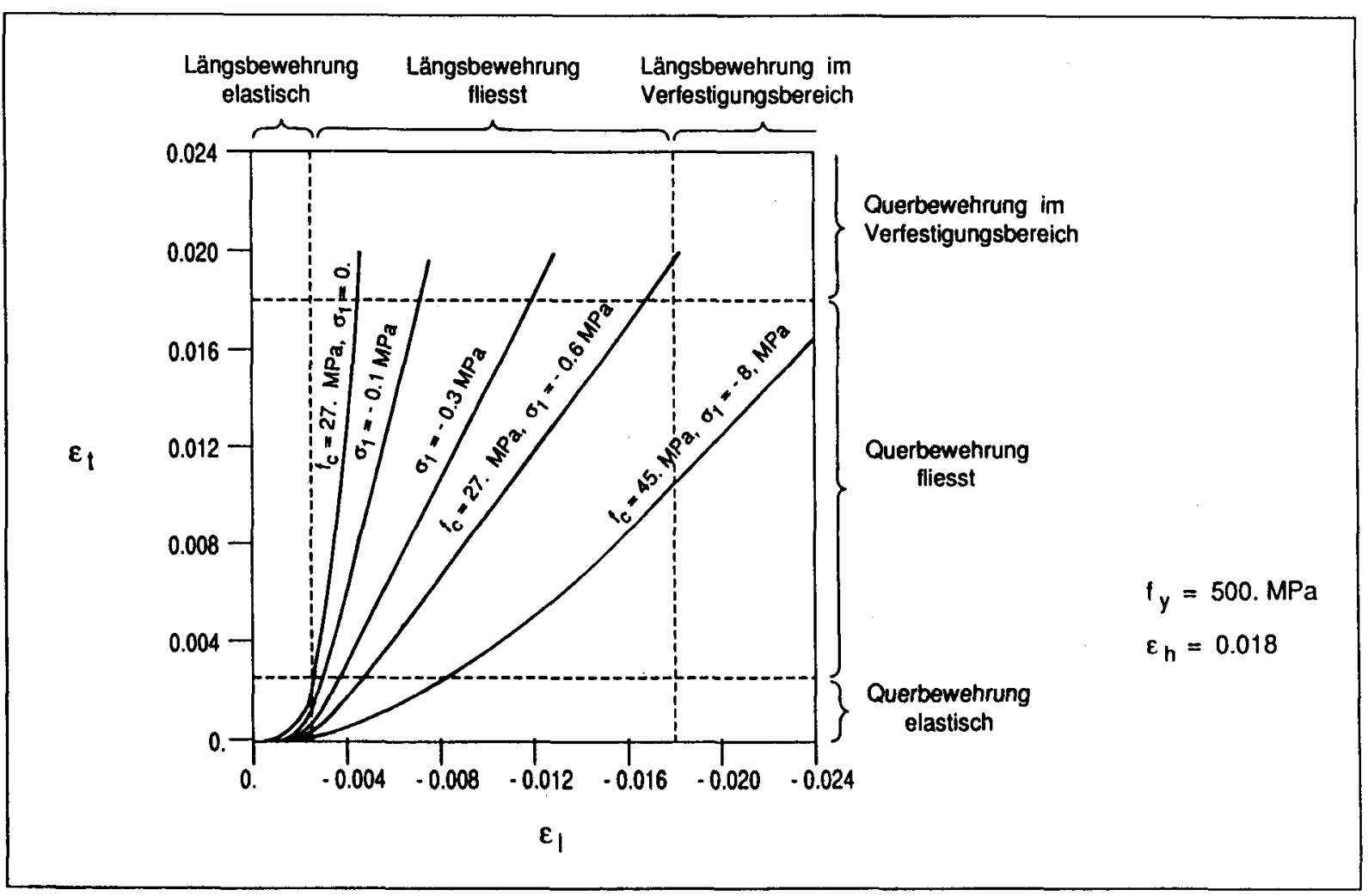

Bild 4.8: Beziehung zwischen Lăngsstauchung und Querdehnung des dreiachsig beanspruchten Betons (Versuche von Kosaka, Tanigawa, Hatanaka (1985) und van Mier (1984)) 


\subsection{Blegung}

Analog den zugbeanspruchten Stahlbetonscheiben (Abschnitt 4.1) erfolgt eine Verformungslokalisierung, wenn die bei der Rissbildung vorhandene Beanspruchung von der Bewehrung nicht aufgenommen werden kann. Den zur Vermeidung dieser Verformungslokalisierung notwendigen minimalen Bewehrungsgehalt $\omega_{\min }$ erhält man aus der Gleichsetzung der Widerstănde im ungerissenen und im gerissenen Zustand.

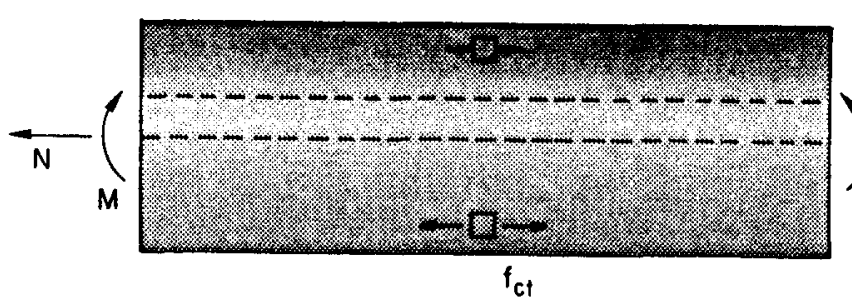

(a) ungerissener Zustand

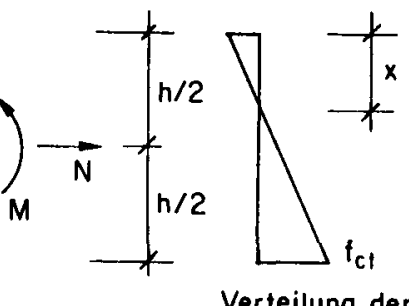

Verteilung der Längsspannung

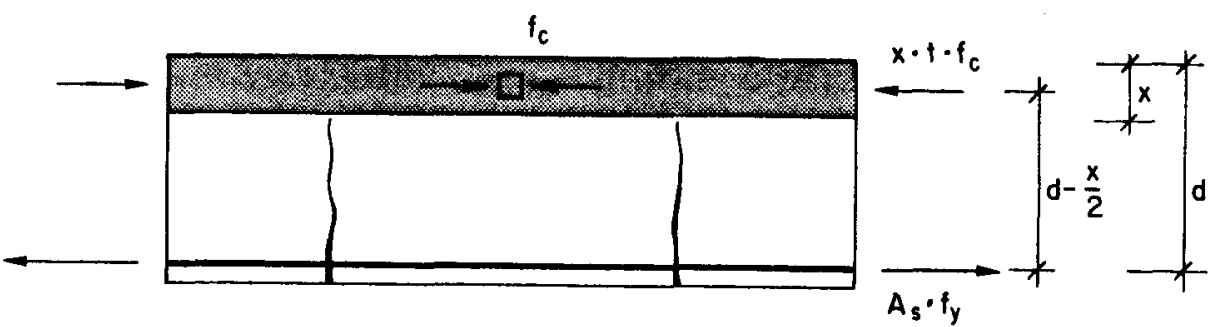

(b) gerissener Zustand

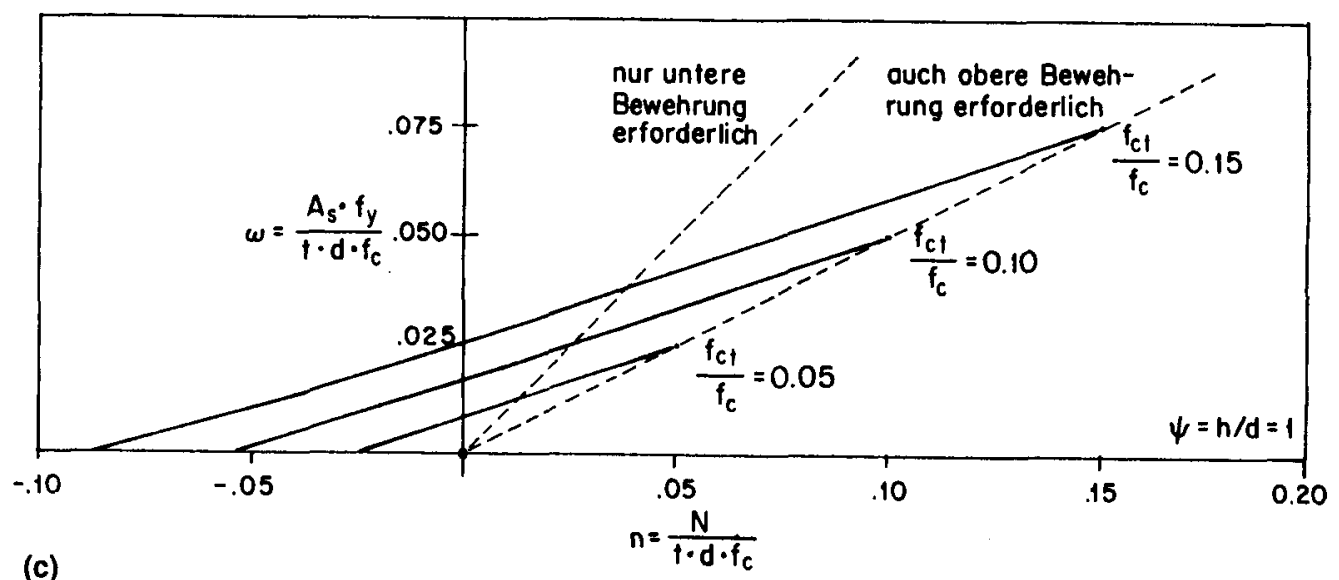

Bild 4.9: Spannungsfeld für die Biegebeanspruchung (a) im ungerissenen und (b) im gerissenen Zustand; (c) minimaler Bewehrungsgehalt zur Verhinderung von Verformungslokalisierungen. 
Die Beanspruchung gemäss Bild 4.9a, die zum Reissen des Betons führt, ergibt sich für einen Rechteckquerschnitt zu

$$
\frac{M}{T} \cdot \frac{h}{2}+\frac{N}{A}=\frac{6 \cdot M}{t \cdot h^{2}}+\frac{N}{t \cdot h}=f c t
$$

und mit den Normienungsgrössen $m=M /\left(t \cdot d^{2} \cdot f_{c}\right), n=N /\left(t \cdot d \cdot f_{c}\right)$ und $\psi=h / d$ :

$$
\frac{6 \cdot m}{\psi^{2}}+\frac{n}{\psi}=\frac{f c t}{c}
$$

Verglichen mit der mit einem Zugversuch an einem Probekörper ermittelten Festigkeit ist der einzusetzende Wert für $\mathrm{f}_{\mathrm{ct}}$ im allgemeinen durch Mikrorisse und Eigenspannungen reduziert.

Nach der Rissbildung ergibt sich das plastische Moment gemäss Bild $4.9 \mathrm{~b}$ zu

$$
m=\omega \cdot\left(1-\frac{\omega}{2}+n\right)-\frac{n}{2} \cdot(\psi+n)
$$

Der minimale Bewehrungsgehalt betrăgt somit

$$
\omega_{\min }=1+n-\sqrt{1+2 \cdot n \cdot\left(1-\frac{\psi}{3}\right)-\frac{f_{c t}}{f_{c}} \cdot \frac{\psi}{3}} \quad \text { für } n \leq \omega
$$

und

$$
\omega_{\min }=\frac{f_{c t} \cdot \frac{\psi^{2}}{6}+n \cdot\left(1-\frac{2 \cdot \psi}{3}\right)}{2-\psi} \quad \text { für } n \geq \omega
$$

Bild 4.2 macht deutlich, dass zur Vermeidung von Verformungslokalisierungen im Gebrauchszustand (breite Risse) mit der Streckgrenze $f_{y}$ als Festigkeitswert gerechnet werden muss, so dass

$$
\omega=\frac{A_{S} \cdot f}{t \cdot d \cdot f_{c}} .
$$

gilt. Um im Bruchzustand die Verformungslokalisierung (Reduktion der Duktilităt, Bild 4.2c) zu vermeiden, kann die Zugfestigkeit $f_{t}$ eingesetzt werden, womit der mechanische Bewehrungsgehalt

$$
\omega=\frac{A_{s} \cdot f \cdot t}{t \cdot d \cdot f_{c}}
$$

resultiert.

Der minimale Bewehrungsgehalt $\omega_{\min }$ ist im Bild $4.9 \mathrm{c}$ graphisch als Funktion von $n$ für verschiedene Betonzugfestigkeiten $f_{c t}$ ausgewertet. Aus dem Bild geht hervor, dass bei grossen Druckkrăften die Risse auch ohne Bewehrung verteilt werden. Für Stützen und vorgespannte Trăger ist dieser Einfluss sehr wichtig. Bild 4.10a zeigt die Rissbilder von vorgespannten Trăgern im Bruchzustand, die von Ivanyi, Buschmeyer (1981) geprüft worden sind. Diese Trăger waren ohne Verbund vorgespannt und enthielten keine schlaffe Bewehrung. Die Rissverteilung erfolgte nur dank der aus Vorspannung resultierenden Druckkraft. 


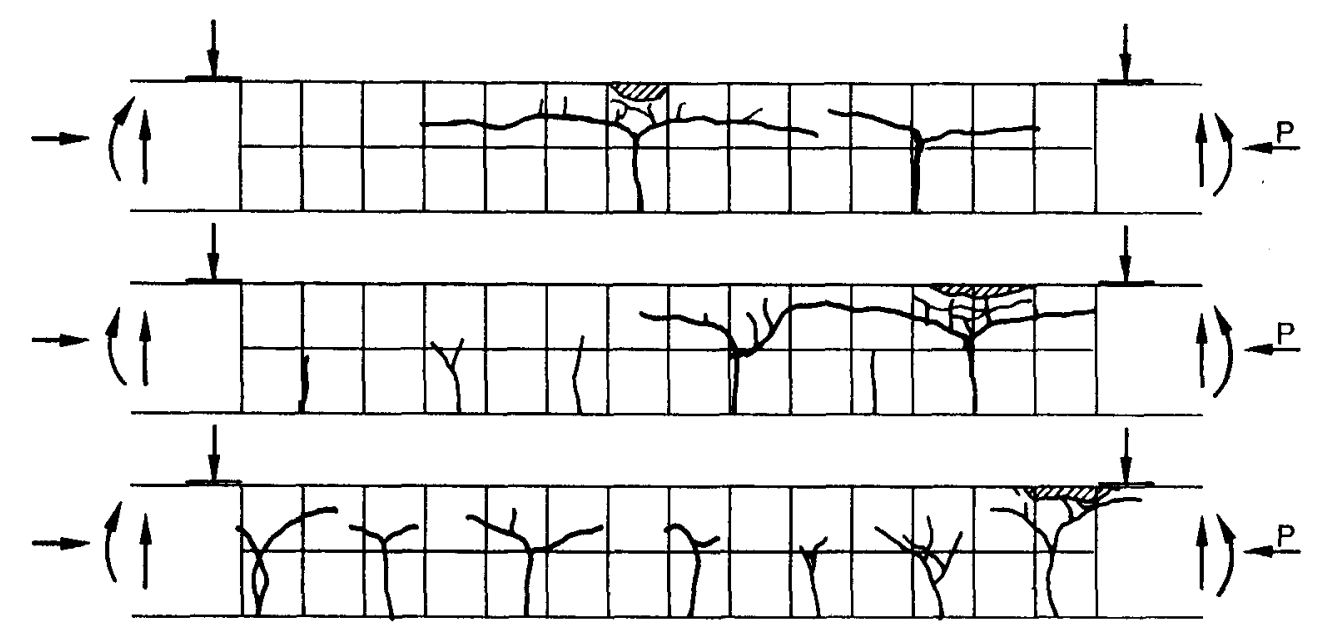

Versuch PS 4

$\frac{P}{t \cdot h}=2 . M P a$

$\mathrm{n}=-0.08$

Versuch PS 5

$\frac{P}{t \cdot h}=3.25 \mathrm{MPa}$

$n=-0.09$

Versuch PS 6

$\frac{P}{t \cdot h}=4.5 \mathrm{MPa}$

$n=-0.12$

(a)

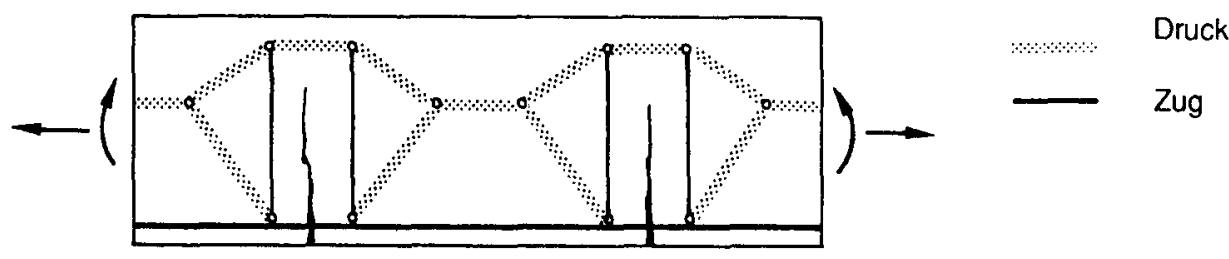

(b)

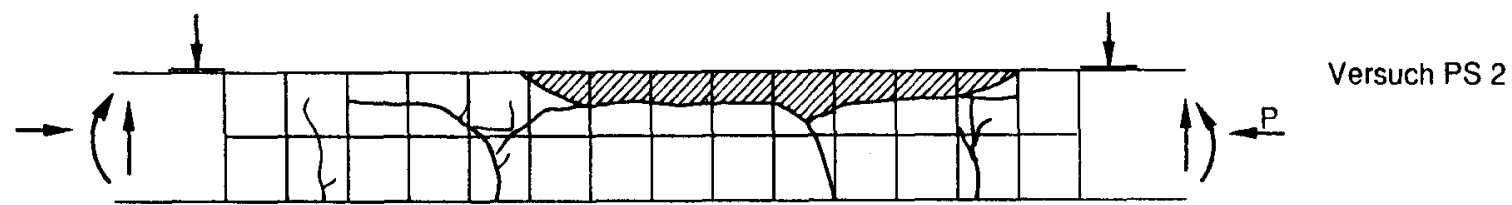

(c)

Der beim Bruch ausgesprengte Beton ist durch

Schraffur gekennzeichnet

Bild 4.10: (a) Rissbilder von ohne Verbund vorgespannten Balken im Bruchzustand (Versuche von Ivanyi, Buschmayer (1981) ohne schlaffe Bewehrung) (b) effektiv vorhandene Tragwirkung bei grossen Rissabstănden; (c) Abplatzung der Druckzone. 
Das im Bild 4.9b dargestellte Spannungsfeld ist ein statisch zulăssiger Zustand gemăss Plastizitătstheorie. Insbesondere in der ganzen Druckzone ist die Betonfestigkeit ${ }_{c} c$ ausgenützt. Wie Kanellopoulos (1986) gezeigt hat, ist die Uebereinstimmung zwischen experimentell und theoretisch ermittelten Biegewiderstănden gut. Das im Bild dargestellte Spannungsfeld weicht vor allem im Bereich zwischen den Rissen vom tatsăchlich sich einstellenden Spannungszustand ab. Bei grossen Rissabstânden bleibt zwischen den Rissen das Spannungsfeld des ungerissenen Zustandes mit einer tieferen Resultierenden der Druckzone erhalten.

Im Bild 4.10b sind die Druck- und Zugresultierenden in der Druckzone eines gerissenen Trägers schematisch dargestellt. Für die Umlenkung der Druckkraft sind Druck- und Zugkräfte quer zur Lăngsachse erforderlich. Nach Buschmeyer (1983) erreicht die Querzugspannung in der Năhe der Risswurzel fast den halben Wert der Lăngsdruckspannung, so dass sich die Risse in horizontaler Richtung fortpflanzen kőnnen, wie dies im Bild 4.10a abgebildet ist. Solche Risse entstehen vor allem, wenn die vertikalen Risse einen grossen Abstand zueinander haben. Ein âhnliches Rissbild zeigen auch die Versuche von Ritz, Marti, Thürlimann (1975) mit vorgespannten Trăgern ohne Verbund und die von Phipps (1976) durchgeführten Druckversuche mit Exzentrizităt. Wenn keine Querbewehrung vorhanden ist, kőnnen diese Lăngsrisse die Druckzone schwächen und damit zu einem vorzeitigen spröden Bruch führen. Bild 4.10c zeigt einen von Ivanyi, Buschmeyer (1981) geprüften Trăger mit Vorspannung ohne Verbund, bei dem die Druckzone abgesprengt ist.

Damit diese spröde Bruchart vermieden werden kann, muss entweder eine Querbewehrung angeordnet werden, oder der Rissabstand muss durch geeignete Wahl von Druckkraft und schlaffer Bewehrung derart klein gehalten werden, dass die Querzugbeanspruchung abnimmt und sich die Risse nicht in Lăngsrichtung fortpflanzen kơnnen. In diesem Fall bricht die Betondruckzone gleich wie beim Zylinderversuch, d.h., es entstehen feine Lăngsrisse, und die Druckzone wird in Lamellen aufgespalten. Ein typisches Bruchbild enthâlt Bild 4.11a (siehe auch Bild 3.8). Bild 4.11b zeigt den von Kanellopoulos (1986) vorgeschlagenen Verzerrungszustand des Verformungsbereichs. Dieser ist gekennzeichnet durch einen Bereich der Druckzone mit konstanter Lăngsstauchung und Querdehnung. Diese Verzerrungen sind vom Rotationswinkel $\varphi$ und von der Neigung $\alpha$ der Diskontinuitătslinien zwischen dem Verformungsbereich und der übrigen Druckzone abhängig:

$$
\begin{aligned}
& \varepsilon_{3}=-\frac{\varphi}{2} \cdot \tan \alpha \\
& \varepsilon_{1}=\frac{\varphi}{2} \cdot \cot \alpha
\end{aligned}
$$

Bei einer plastischen Verformung ist das Fliessgesetz (Normalităt zwischen Fliessfigur und Verzernungsvektor) ertültt für

$$
\frac{\varepsilon_{1}}{\varepsilon_{3}}=-\frac{\sigma_{3}}{\sigma_{1}} \quad=-4, \text { d.h. mit } \tan \alpha=\frac{1}{2}
$$


(a)
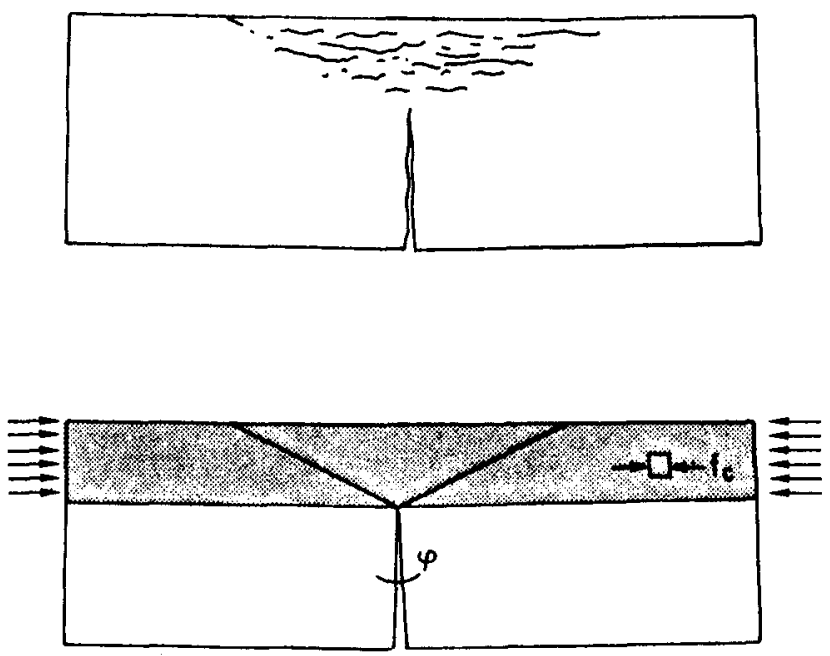

(b)

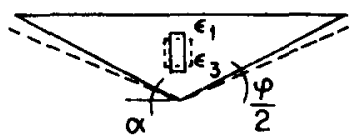

Bild 4.11: (a) Rissbild der Biegedruckzone; (b) Verformungszustand nach Kanellopoulos (1986).

Dass die Verzerrungen im dreieckförmigen Verformungsbereich etwa konstant sind, wurde auch von lqbal, Hatcher (1975) experimentell bestätigt. Bei grossen Rotationen $\varphi$ entfestigt der dreieckförmige Verformungsbereich. Da mit zunehmender Verformung die Bewehrungskraft und somit auch die Betondruckkraft nicht abnehmen können, werden weitere Bereiche für die Aufnahme der Druckkraft aktiviert (Bild 4.12). Der neu aktivierte Bereich ist zweiachsig oder dreiachsig beansprucht, so dass die Festigkeit höher als ${ }_{c}{ }_{c}$ sein kann. Der innere Hebelarm hat aber in diesem Bereich abgenommen, so dass eine Abminderung der Biegebeanspruchung resultiert. Im Bild $4.12 \mathrm{c}$ ist das aufnehmbare Moment in Funktion der Betonspannung im entfestigenden Bereich der Druckzone für verschiedene mechanische Bewehrungsgehalte $\omega$ graphisch ausgewertet. Die mögliche Zunahme der Festigkeit im mehrachsig beanspruchten Teil der Druckzone wird vernachlässigt; dort wird jedoch ein entfestigungsfreies Verhalten angenommen. Aus dem Bild 4.12 geht klar hervor, dass der Einfluss der Betonentfestigung auf das Moment mit dem Bewehrungsgehalt stark zunimmt. Die im Bild 4.13 dargestellten Last-Vertormungs-Kurven der von Base, Read (1965) und lqbal, Hatcher (1975) durchgeführten Biegeversuche bestätigen diese Abhängigkeit. Die stark bewehrten Träger weisen eine rasche Entfestigung auf. 
(a)
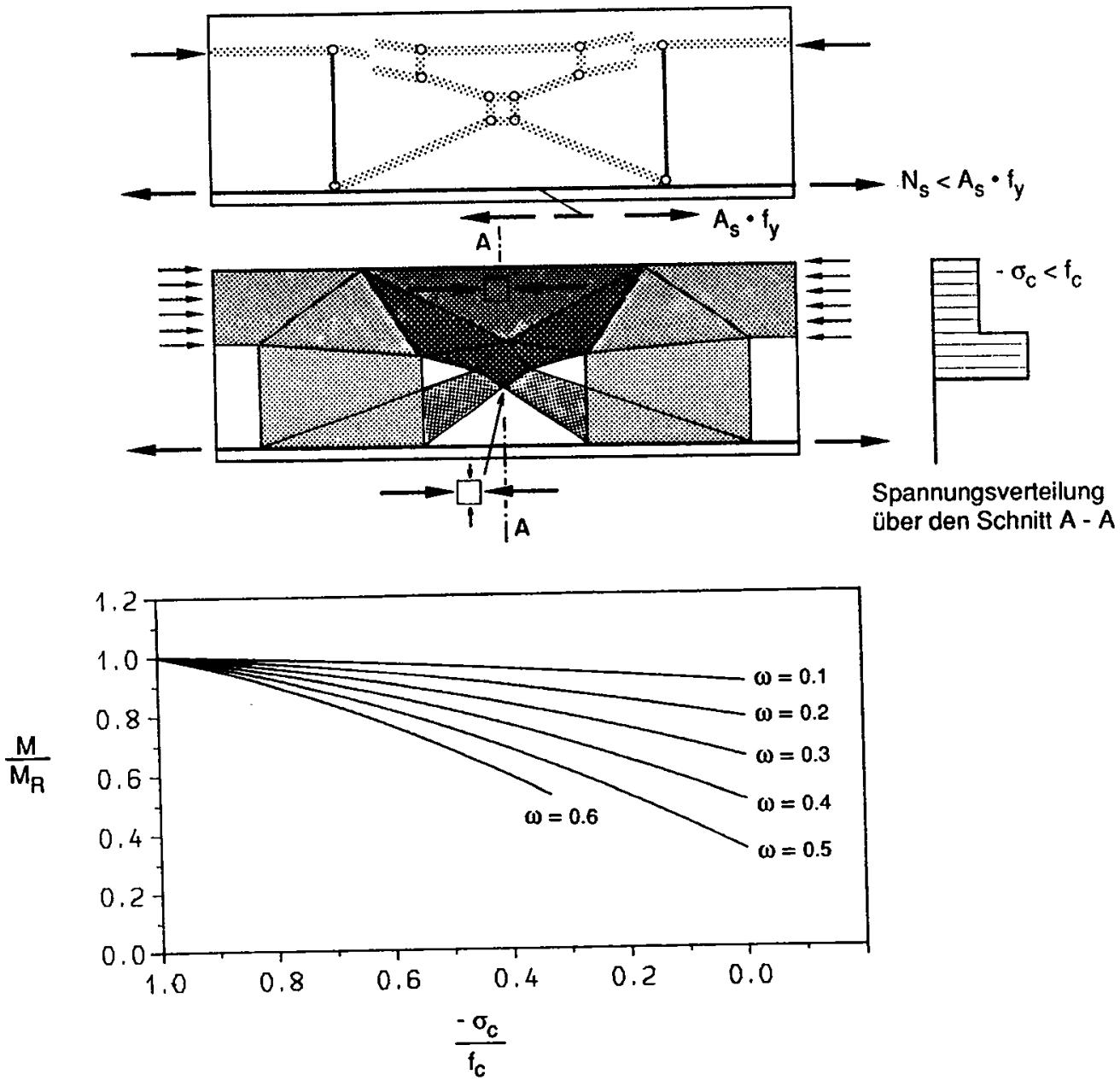

(c)

Bild 4.12: Tragwirkung im Verformungsbereich eines Biegeträgers nach der Entfestigung der Druckzone: (a) Darstellung mit den Resultierenden; (b) Darstellung mit einem Spannungsfeld (das im Sinn der Plastizitătstheorie entwickelte Spannungsfeld gibt nur Auskunft über den qualitativen Verlauf der inneren Kräfte); (c) aufnehmbares Moment als Funktion der Betonspannung im entfestigenden Bereich. 


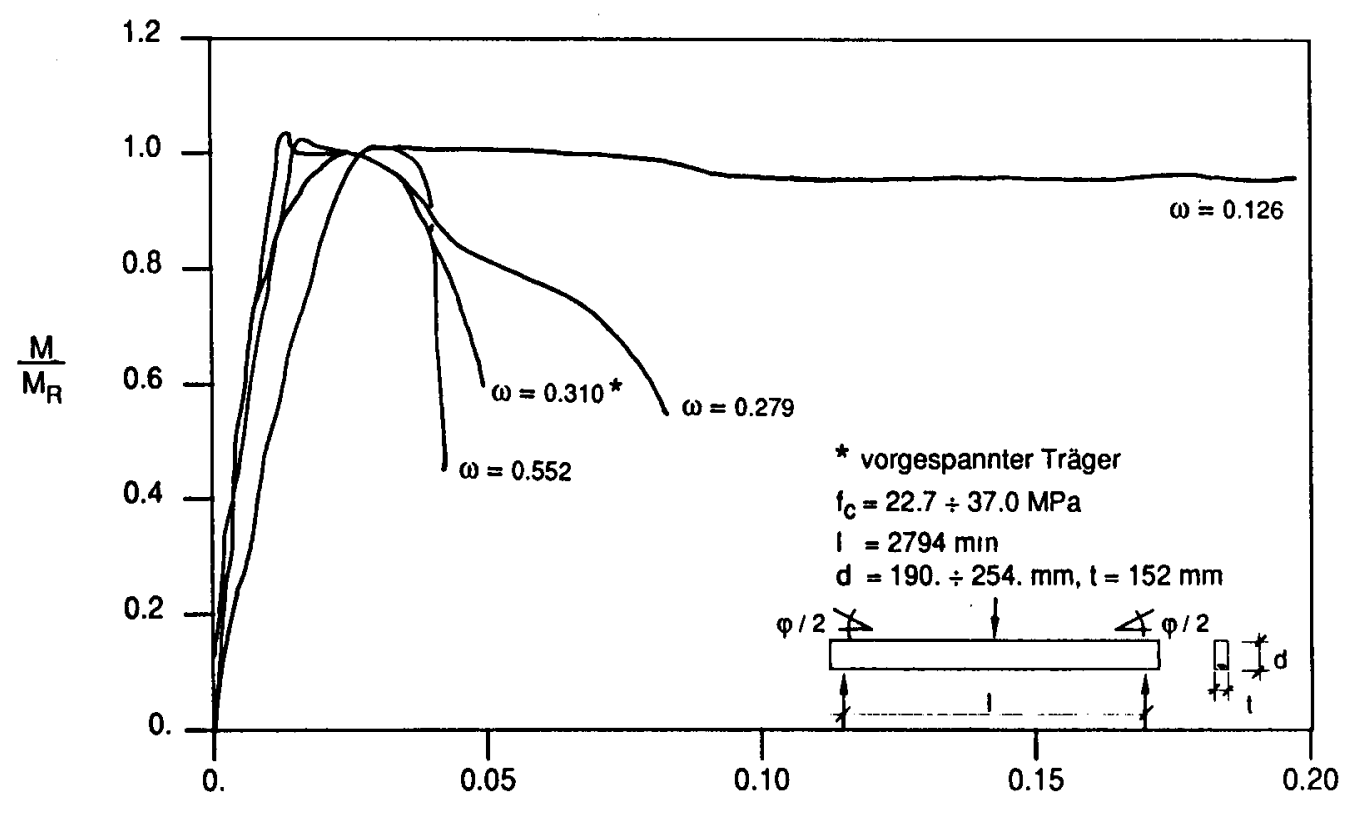

(a)

$\varphi$ [ rad]

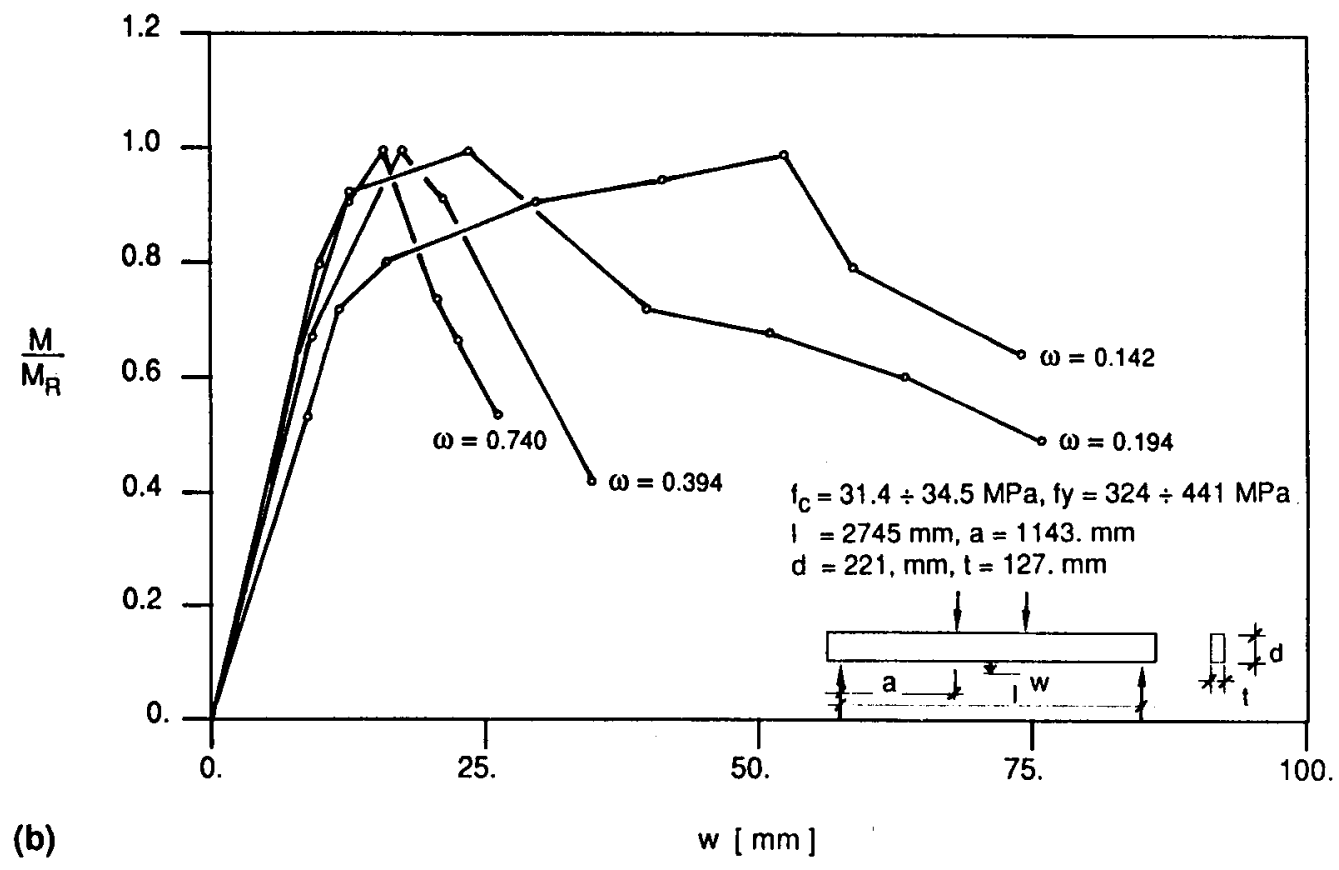

Bild 4.13: Last-Verformungs-Beziehung von Biegeversuchen mit variablem Långsbewehrungsgehalt: (a) Versuche von Base, Read (1965) mit sehr kleinem Bügelbewehrungsgehalt; (b) Versuche von Iqbal, Hatcher (1975) ohne Bügelbewehrung. 
(a)

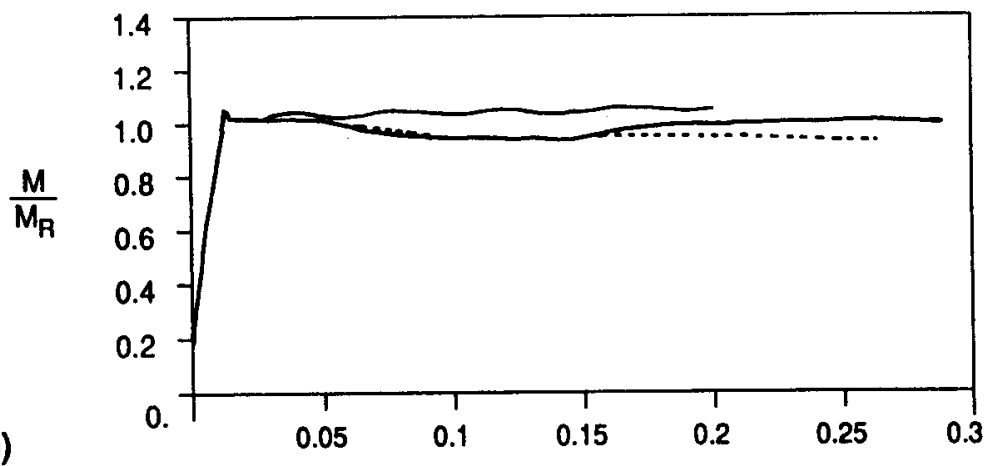

$\omega=0.100 \div 0.126$

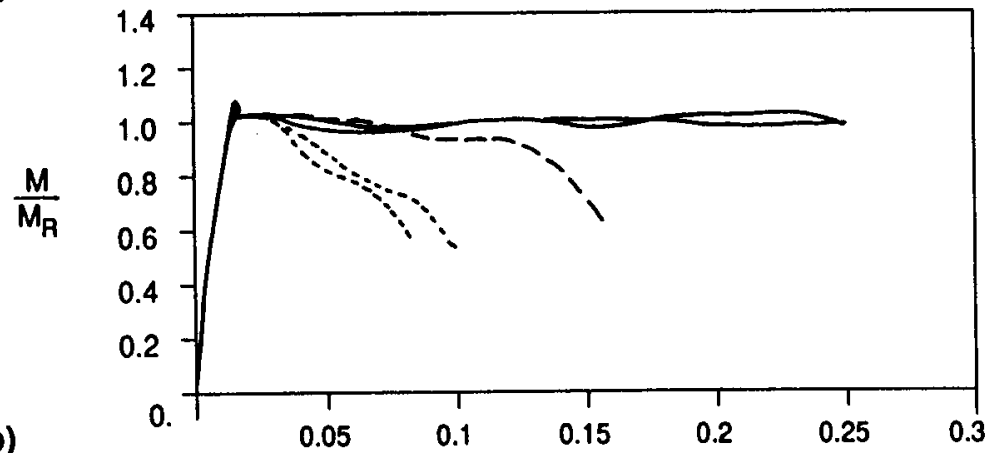

$\omega=0.200 \div 0.285$

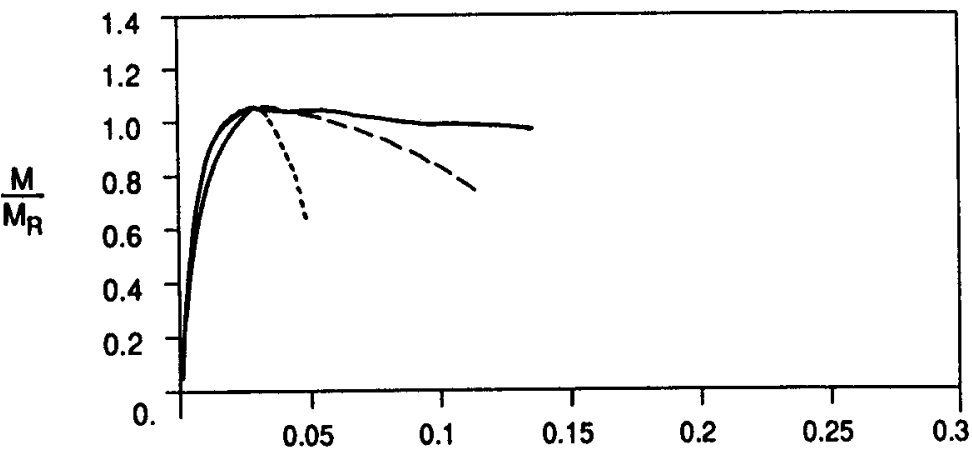

$\omega=0.239 \div 0.310$

(vorgespannt)

(c)

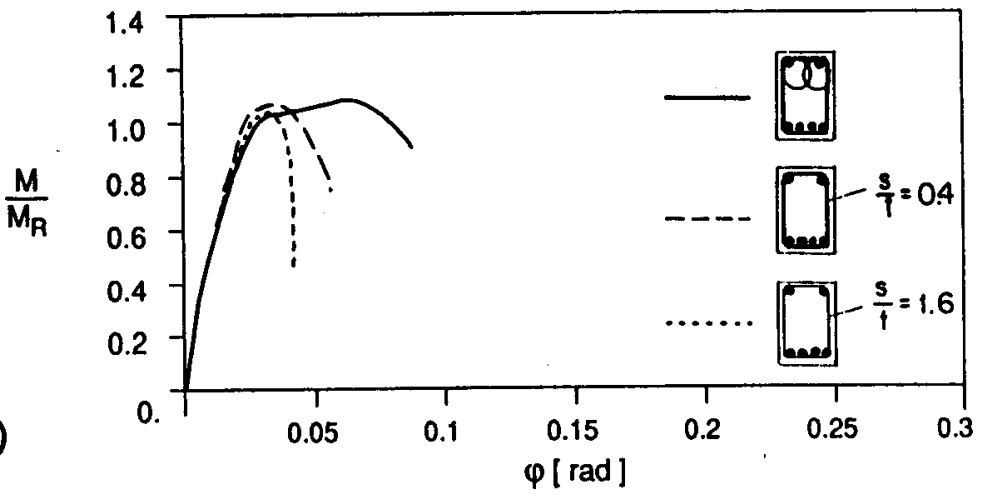

$\omega=0.520 \div 0.676$

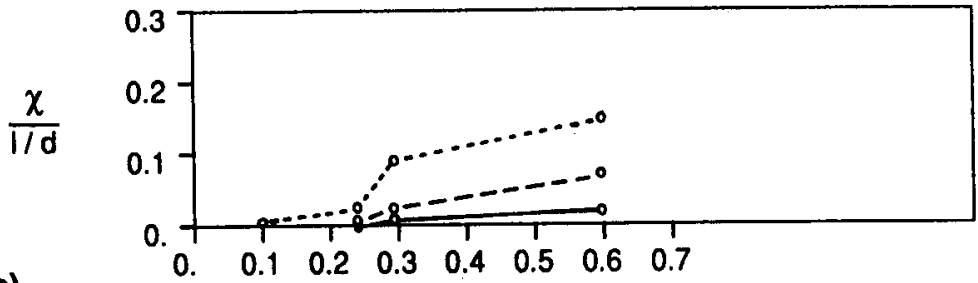

(e)

Bild 4.14: Einfluss der Querbewehrung auf die Last-Verformungs-Beziehung der Biegeversuche von Base, Read (1965): (a) bis (d) Momenten-Rotations-Beziehung; (e) Entfestigungsstabilitătsfaktor. 
(a)
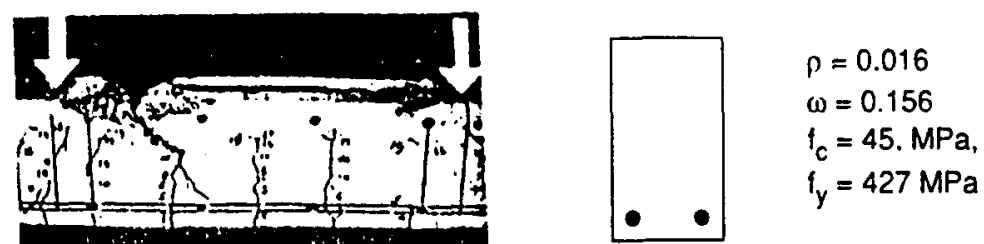

(b)
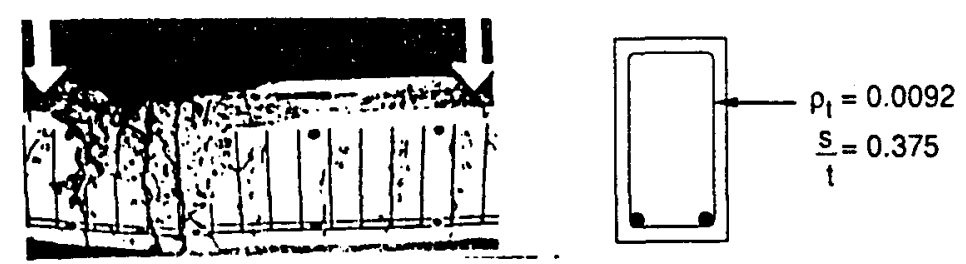

(c)
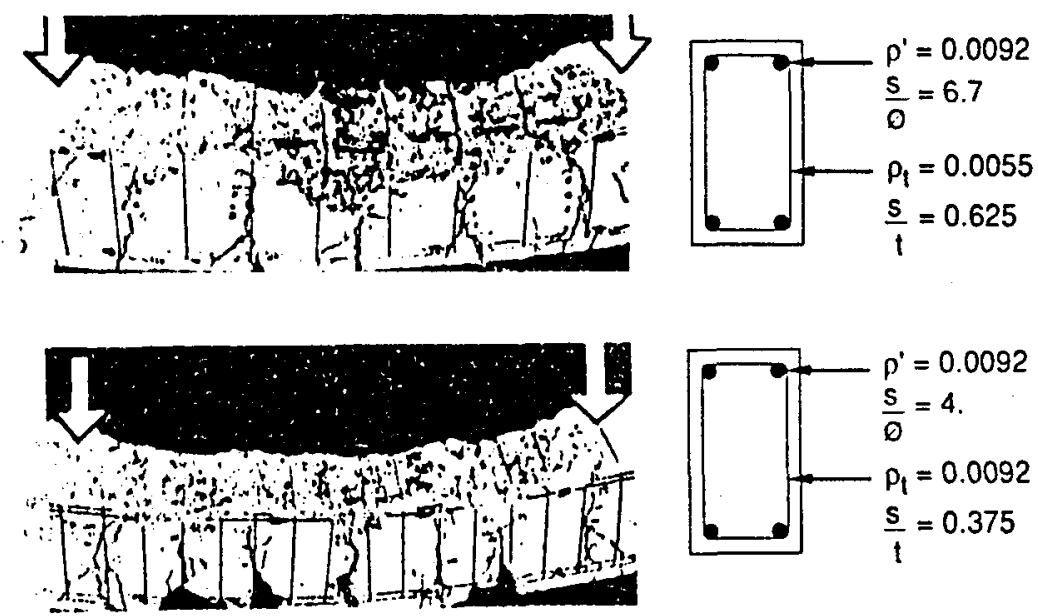

(d)

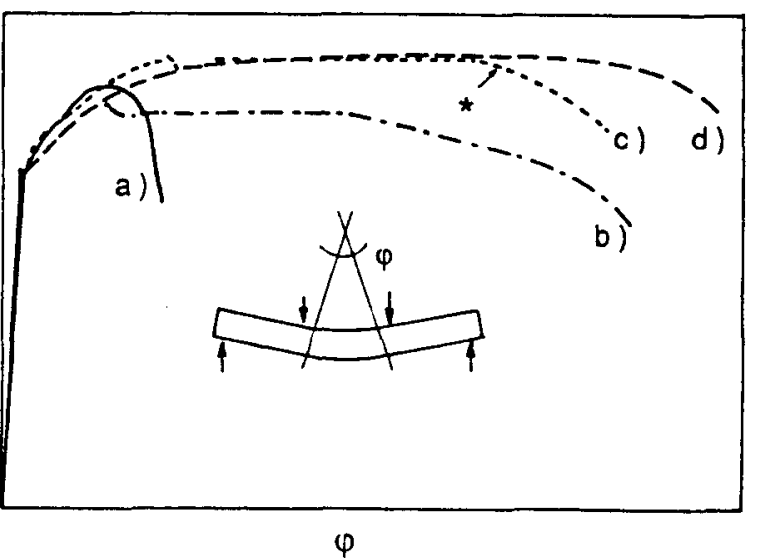

* Knicken der Druckbewehrung

M

(e)

Bild 4.15: Einfluss der Querbewehrung und der Druckbewehrung auf die Lokalisienung der Verformungen und auf die Momenten-Rotations-Beziehung in den Versuchen von Bertero, Felippa (1964). 
Für die Duktilităt der Druckzone und somit der Biegetrăger gelten die gleichen Ueberlegungen wie für die im Abschnitt 4.2 behandelten Druckglieder. Die Querbewehrung (Bügel) und die Lăngsbewehrung in der Druckzone erhőhen auch hier die Duktilităt stark.

Der Einfluss der Querbewehrung ist anhand der im Bild 4.14 dargestelten Momenten-RotationsBeziehungen der von Base, Read (1965) durchgeführten Versuche klar ersichtlich. Balken mit vier unterschiedlichen Lăngsbewehrungsgehalten wurden mit Bügeln mit grossem $A b s t a n d ~(s / t=1.6)$, mit Bügeln mit kleinem Abstand $(s / t=0.4)$ oder mit einer Spirale in der Druckzone $(s / t=0.2,0.4)$ bewehrt.

Besonders effizient ist die spiralförmige Bewehrung in der Druckzone. Bei Balken mit $q_{\mathfrak{L}} \simeq 0.5$ konnte damit der Entfestigungsstabilitătsfaktor von $0.15 \mathrm{zu} 0.07$ und 0.01 (für grossen resp. engen Bügelabstand) reduziert werden.

Eine starke Druckbewehrung verbessert das Verhalten, weil die Druckzone entlastet wird. Besonders günstig ist der Einfluss der Druckbewehrung, wenn sie bis zur Verfestigung gestaucht werden kann. Wie die Versuche von Bertero, Felippa (1964) zeigen (Bild 4.15), kann in diesem Fall eine Verformungslokalisierung verhindert werden (die Stahlvertestigung kompensiert die Betonentfestigung), so dass das Verhalten deutlich verbessert wird. Aehnlich wie bei den Druckgliedern können grosse Stauchungen der Druckbewehrung nur erreicht werden, wenn diese mit der Querbewehrung genügend stabilisiert wird.

\subsection{Schub}

In diesem Abschnitt werden auf Schub beanspruchte Elemente betrachtet, deren Bewehrung so bemessen ist, dass die Risse verteilt werden. Aehnliche Elemente, jedoch ohne Schubbewehrung, werden im Kapitel 6 behandelt.

Als erstes Beispiel wird das Last-Verformungs-Verhalten eines auf Schub beanspruchten Scheibenelementes gemåss Bild 4.16a entwickelt. Dabei werden folgende Annahmen getroffen:

- Der Beton befindet sich in gerissenem Zustand. Die Risse öffnen sich nur senkrecht zur Rissrichtung (keine Schiebung der Risse), so dass der Beton einachsig auf Druck beansprucht ist.

- Die Reduktion der Betonfestigkeit infolge Querdehnung wird berücksichtigt, indem eine effektive Festigkeit $f_{c e}$ gemåss Abschnitt 3.2 (Bild 3.13c) eingesetzt wird. Die im Bild 3.10 für den Beton angegebene Spannungs-Dehnungs-Beziehung wird übernommen, wobei die Spannung im Verhältnis $\mathrm{f}_{c e}{ }^{f} c$ reduziert wird.

- Die Hauptrichtung des Dehnungstensors stimmt mit derjenigen des Spannungstensors überein (die Querdehnung ist senkrecht zur Betondruckspannung, frühere Neigungen des Druckfeldes werden nicht berücksichtigt). 
- Die Bewehrung nimmt nur Zugkrăfte auf. Der Stababstand ist gegenüber den Abmessungen der Scheibe derart klein, dass die Wirkung der Bewehrung auf dem Beton als konstant verteilt betrachtet werden kann.

- Der Beton nimmt auch zwischen den Rissen keine Zugkraft auf, so dass für die Bewehrung die Spannungs-Dehnungs-Beziehung des Stahles gilt.

Die Schubbeanspruchung des Scheibenelementes $\tau_{x y} \cdot t$ ist ăquivalent der Summe der Betonbeanspruchung und der Beanspruchungen

$$
\bar{\sigma}_{x}=\sigma_{s x} \cdot \rho_{x} \quad \text { und } \quad \bar{\sigma}_{y}=\sigma_{s y} \cdot \rho_{y}
$$

in den verschmiert gedachten Bewehrungen in $x$ - und y-Richtung (Bild 4.16b).

Die Gleichgewichtsbedingungen können aus der Mohrschen Darstellung des Spannungszustandes (Bild 4.16c) gewonnen werden:

$$
\begin{aligned}
\tan ^{2} \vartheta & =\frac{\bar{\sigma}_{y}}{\bar{\sigma}_{x}} \\
\tau_{x y} & =\sqrt{\bar{\sigma}_{x} \cdot \bar{\sigma}_{y}} \\
\sigma_{c} & =-\left(\bar{\sigma}_{x}+\bar{\sigma}_{y}\right)
\end{aligned}
$$

Bild 4.16d zeigt die Mohrsche Darstellung des Verzerrungszustandes. Daraus lassen sich folgende kinematische Relationen ermitteln:

$$
\begin{aligned}
\tan ^{2} \vartheta & =\frac{\varepsilon_{x}-\varepsilon_{3}}{\varepsilon_{y}-\varepsilon_{3}} \\
\frac{\gamma_{x y}}{2} & =\sqrt{\left(\varepsilon_{x}-\varepsilon_{3}\right) \cdot\left(\varepsilon_{y}-\varepsilon_{3}\right)} \\
\varepsilon_{1} & =\varepsilon_{x}+\varepsilon_{y}-\varepsilon_{3}
\end{aligned}
$$

Das Last-Verformungs-Verhalten des Scheibenelementes erhält man aus der Lösung des Gleichungssystems, bestehend aus den Gleichgewichtsbedingungen, den Verträglichkeitsbedingungen und den im Bild 4.16d graphisch dargestellten Stoffgesetzen. 


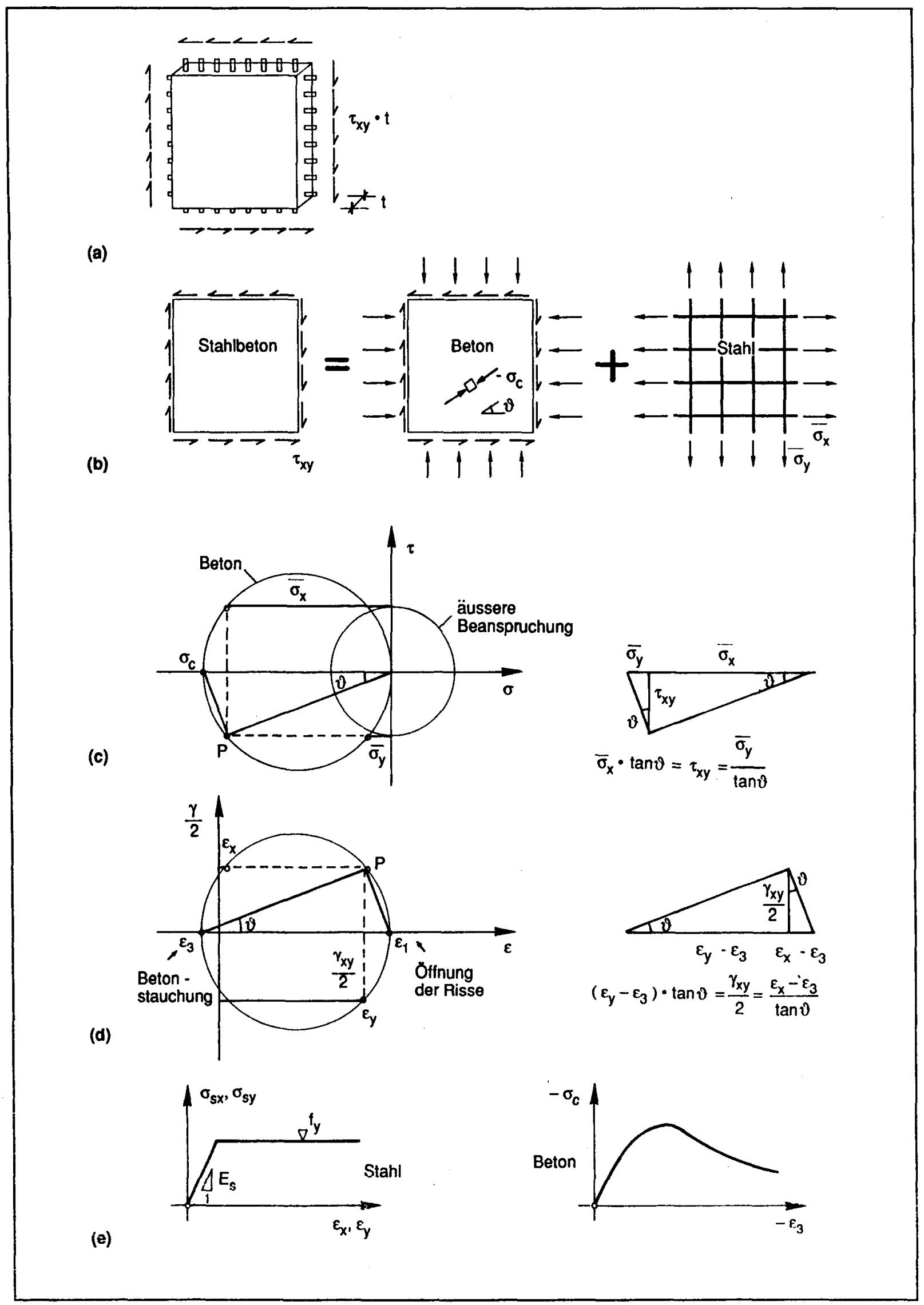

Bild 4.16: (a) Schubbeanspruchtes Scheibenelement; (b) Scheibenbeanspruchung als Superposition der Beton- und Stahlbeanspruchung; (c) Mohrsche Darstellung des Spannungszustandes; (d) Mohrsche Darstellung des Verzerrungszustandes; (e) Stoffgesetze. 


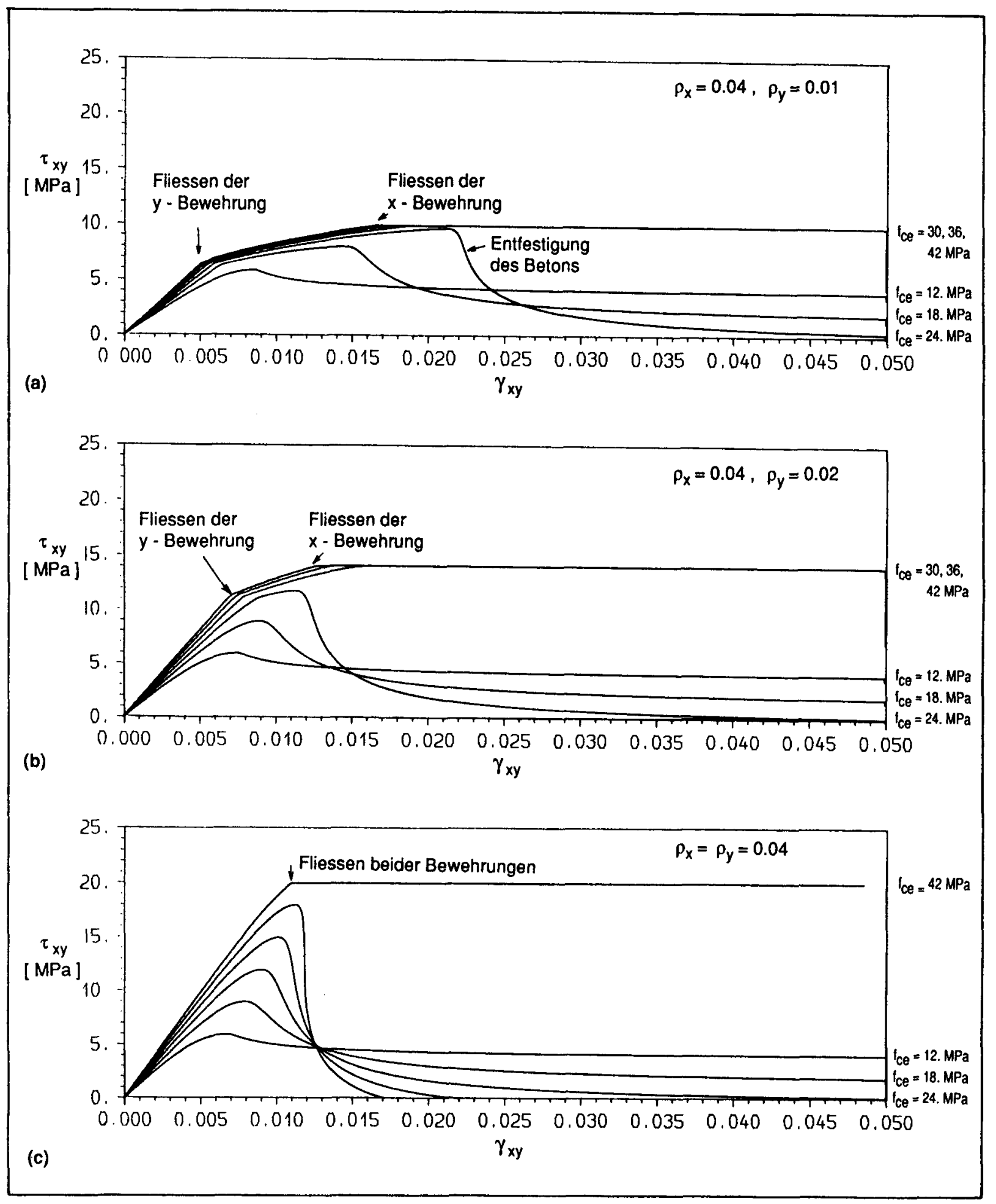

Bild 4.17: Last-Verformungs-Verhalten eines schubbeanspruchten Scheibenelementes (Verformungslokalisierungsbereich); $f_{c e}=12,18,24,30,36,42 \mathrm{MPa} ; f_{y}=500 \mathrm{MPa}$. 
Bild 4.17 zeigt die iterativ ermittelten Last-Verformungs-Kurven für drei Bewehrungskombinationen und verschiedene Werte von $f_{c e}$. Im Bild $4.17 a$ sind die verschiedenen Regimes für die stark anisotrope Bewehrung $\left(\rho_{x} / \rho_{y}=4\right)$ klar ersichtlich. Bei kleinen Beanspruchungen verhalten sich beide Bewehrungsscharen elastisch (Regime B). Wenn die vertikale Bewehrung (in diesem Fall die schwächere Bewehrung) die Fliessgrenze erreicht, findet eine Umlagerung der inneren Krăfte statt. Bei einer weiteren Erhöhung der Last nimmt die Neigung des Druckfeldes ab, so dass die Spannung in der horizontalen Bewehrung überproportional zunimmt, wăhrend die vertikale Bewehrung fliesst (Regime BS). Die Traglast wird erreicht, wenn beide Bewehrungsscharen fliessen (Regime S). Wird in einem der ersten beiden Regimes die effektive Festigkeit im Beton erreicht, kann die Last nicht weiter erhöht werden. Die Entfestigung des Betons verursacht eine Entfestigung der Scheibe und eine Verformungslokalisierung gemăss Bild 4.18.

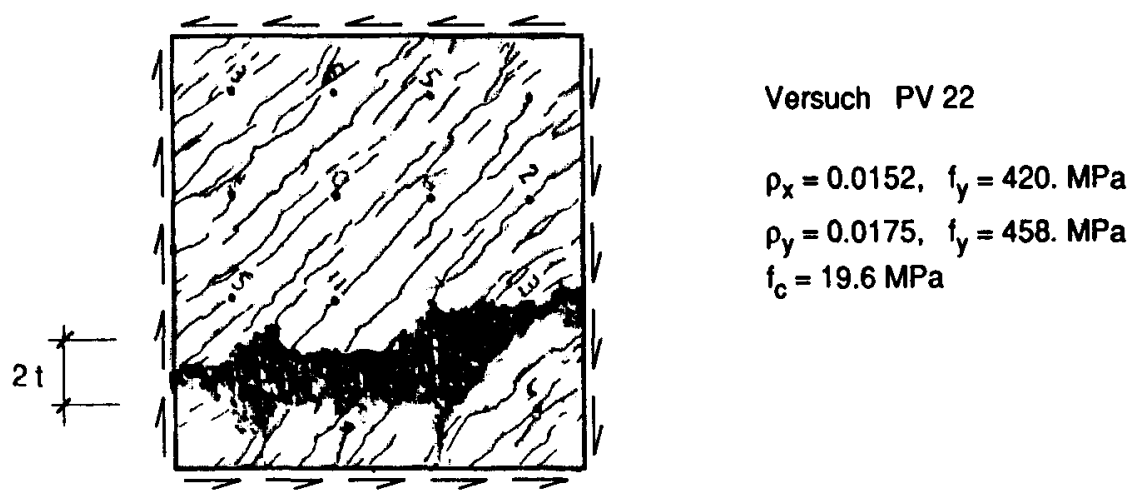

Bild 4.18: Bruchbild mit Verformungslokalisierung aus einem Schubversuch von Vecchio und Collins (1982)

Die absteigenden Aeste der im Bild 4.17 dargestelten Last-Verformungs-Kunen beziehen sich auf den Bereich der Verformungslokalisienung. Für die Charakteristik der Scheibe muss berücksichtigt werden, dass im restlichen Bereich eine Entlastung enfolgt. Nach einem Betonbruch resultiert somit für die Scheibe praktisch immer eine Entfestigungsinstabilităt.

Es ist zu bemerken, dass die Traglast der Scheibe für fast alle Regimes mit der Plastizitătstheorie ermittelt werden kann. Der im Bild 4.16 dargestellte Spannungszustand entspricht der Anwendung des unteren Grenzwertsatzes (statischer Grenzwertsatz). Werden die Stahl- und Betonfestigkeiten in den Gleichgewichtsbedingungen eingesetzt, kann die Traglast sofort bestimmt werden. Folgende bereits erwähnte Regimes können unterschieden werden: 
- Regime S (Stahlfliessen)

- die horizontale Bewehrung fliesst: $\quad \bar{\sigma}_{x}=f_{y} \cdot \rho_{x}$

- die vertikale Bewehrung fliesst: $\quad \bar{\sigma}_{y}=f_{y} \cdot \rho_{y}$

Traglast: $\quad \tau_{x y R}=\sqrt{\bar{\sigma}_{x} \cdot \bar{\sigma}_{y}}=f_{y} \cdot \sqrt{\rho_{x} \cdot \rho_{y}}=f_{c} \cdot \sqrt{\omega_{x} \cdot \omega_{y}}$

Druckfeldneigung: $\quad \tan \vartheta=\sqrt{\bar{\sigma}_{y} / \bar{\sigma}_{x}}=\sqrt{\rho_{y} / \rho_{x}}=\sqrt{\omega_{y} / \omega_{x}}$

Betonspannung: $\quad \sigma_{c}=-\left(\bar{\sigma}_{x}+\bar{\sigma}_{y}\right)=-f_{y} \cdot\left(\rho_{x}+\rho_{y}\right)=-f_{c} \cdot\left(\omega_{x}+\omega_{y}\right)$

- Regime BS (Betonbruch wăhrend Stahlfliessen)

- die vertikale Bewehrung fliesst: $\quad \bar{\sigma}_{y}=f_{y} \cdot \rho_{y}$

- der Beton bricht: $\quad \sigma_{c}=-\left(\bar{\sigma}_{x}+\bar{\sigma}_{y}\right)=-f_{c e}$

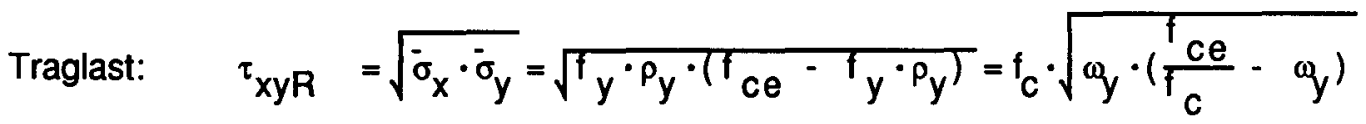

Druckfeldneigung: $\quad \tan \vartheta \quad=\sqrt{\sigma_{y} / \sigma_{x}}=\sqrt{\frac{f}{f_{c e} \rho^{-} \rho_{y}}}=\sqrt{\frac{\omega_{y}}{c e^{\prime f} c^{-} \omega_{y}}}$

oder:

- die horizontale Bewehrung fliesst: $\quad \bar{\sigma}_{x}=f_{y} \cdot \rho_{x}$

- der Beton bricht: $\quad \sigma_{c} \quad=-\left(\bar{\sigma}_{x}+\bar{\sigma}_{y}\right)=-f_{c e}$

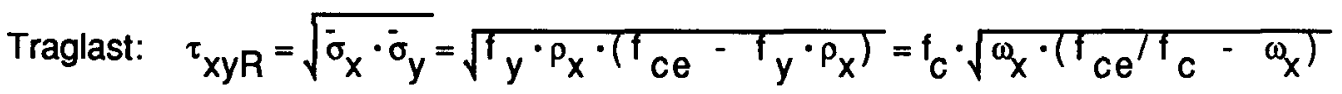

Druckfeldneigung: $\tan \vartheta=\sqrt{\bar{\sigma}_{y} / \bar{\sigma}_{x}}=\sqrt{\frac{f_{c e}-f_{y} \cdot \rho_{x}}{f_{y} \cdot \rho_{x}}}=\sqrt{\frac{f c e^{/ f} c-\omega_{x}}{\omega_{x}}}$

- Regime B (Betonbruch vor dem Stahlfliessen)

- der Beton bricht: $\quad \sigma_{c}=-\frac{\tau x y R}{\operatorname{sin\vartheta } \cos \vartheta}=-f_{c e}$

Traglast: $\quad \tau_{x y R}=f_{c e} \cdot \sin \vartheta \cdot \cos \vartheta$

Die höchste Traglast (unterer Grenzwert) wird für $\vartheta=45^{\circ}$ erreicht:

$$
\tau_{x y R}=\frac{f \text { ce }}{2}
$$




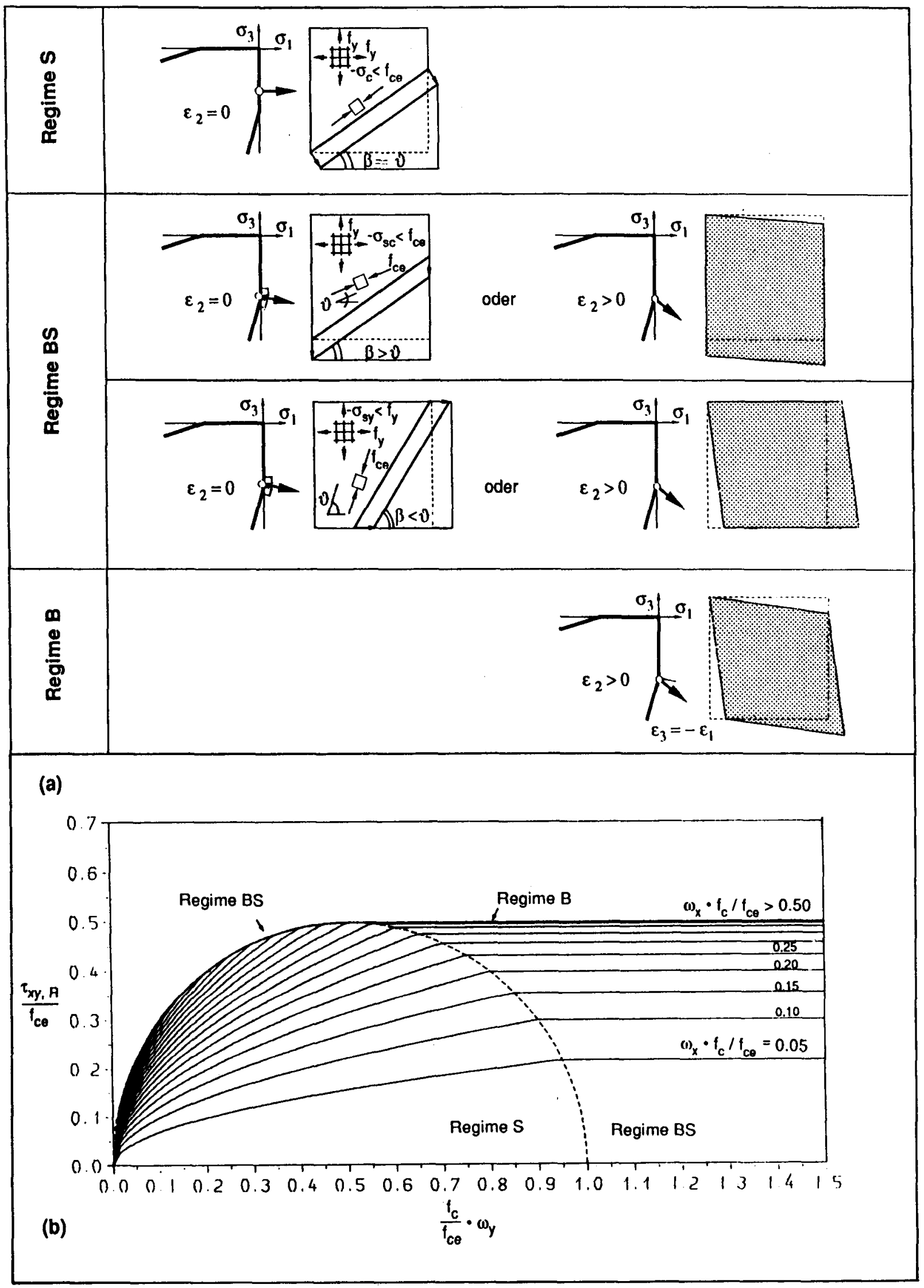

Bild 4.19: (a) Spannungsfelder und Mechanismen der drei Regimes; (b) Traglast als Funktion der mechanischen Bewehrungsgehalte. 
Die angegebenen Lösungen sind vollständige Lơsungen gemäss der Plastizitătstheorie. Bild 4.19a zeigt die entsprechenden Mechanismen. Die plastische Verformung wird entweder in einer infinitesimalen Zone konzentriert (sogenannte Diskontinuitătslinie des Verschiebungsfeldes) oder über das ganze Scheibenelement homogen verteilt. Im Bild $4.19 \mathrm{~b}$ ist die Traglast als Funktion der mechanischen Bewehrungsgehalte graphisch ausgewertet. Weitere Angaben zu dieser Betrachtungsweise können den Arbeiten von Lampert (1970), Thürlimann, Grob, Lüchinger (1975), Müller (1978), Marti (1980) und Nielsen (1984) entnommen werden.

Ein nicht ideal plastisches Verhalten des Betons beeinflusst die Traglast nur im Regime B. Falls die effektiv vorhandene Neigung des Betondruckfeldes beim Erreichen der Betondruckfestigkeit kleiner oder grösser als $45^{\circ}$ ist, bricht das Scheibenelement unter einer Schubbeanspruchung, die kleiner ist als die gemäss Plastizitätstheorie ermittelte Festigkeit. Im Regime BS ist nach Erreichen der Fliessgrenze der schwächeren Bewehrung das Problem innerlich statisch bestimmt geworden, so dass die Druckfeldneigung und damit auch die Traglast von den Steifigkeitsverhăltnissen und von den Zwăngungen unabhăngig sind. Ist die Betonfestigkeit bekannt, kann somit die Traglast mit der Plastizitătstheorie bestimmt werden. Beim Regime $S$ liefert die Plastizitătstheorie ebenfalls korrekte Resultate, weil der Beton nicht bricht.

Bei schubbeanspruchten Balkenstegen können die innere Tragwirkung, das Last-Verformungs-Verhalten und die Traglast analog dem untersuchten Scheibenelement entwickelt werden. Bild 4.20 zeigt ein Beispiel mit dem zugehörigen Spannungsfeld für die Beschreibung der inneren Tragwirkung. Die inneren Beanspruchungen können aus dem Gleichgewicht der auf den im Bild 4.20c dargestellten Schnitt wirkenden Krăfte ermittelt werden:

- Spannung in der Bügelbewehrung:

$$
\begin{aligned}
& \sigma_{s y} \cdot \rho_{y} \cdot t \cdot z \cdot \cot \vartheta=V \\
& \sigma_{s y} \cdot \rho_{y}=\frac{\sigma_{s y}}{f y} \cdot f_{c} \cdot \omega_{y}=\frac{V}{t \cdot z} \cdot \tan \vartheta=\tau_{x y} \cdot \tan \vartheta
\end{aligned}
$$

- Kraft im Zuggurt:

$$
\begin{aligned}
& N_{\text {sup }} \cdot z-V \cdot \frac{Z}{2} \cdot \cot \vartheta=V \cdot \Delta x=-M \\
& N_{\text {sup }}=-\frac{M}{z}+\frac{V}{2} \cdot \cot \vartheta
\end{aligned}
$$


(a)

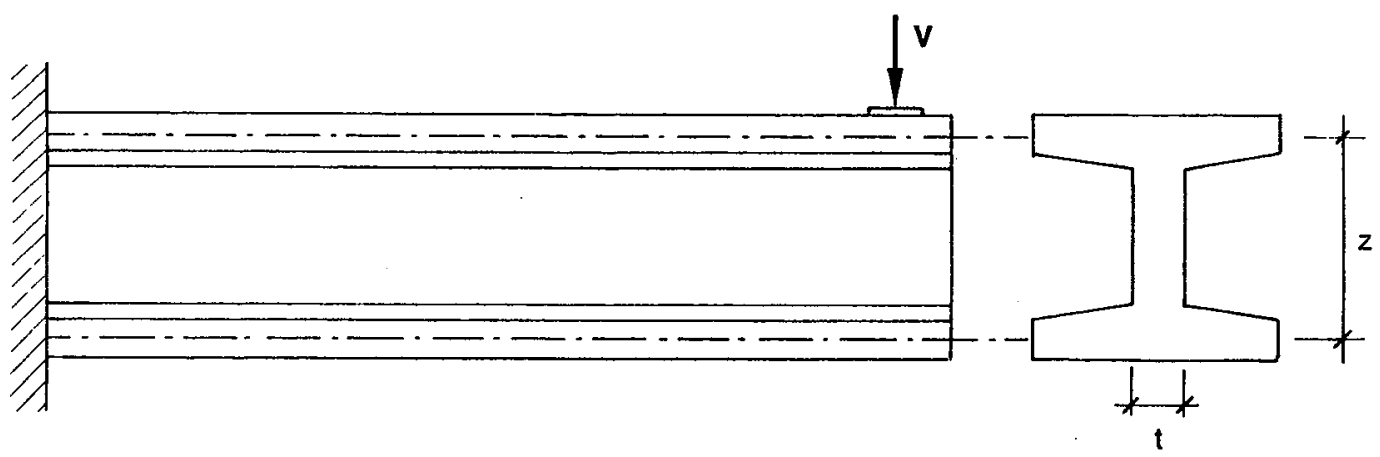

(b)
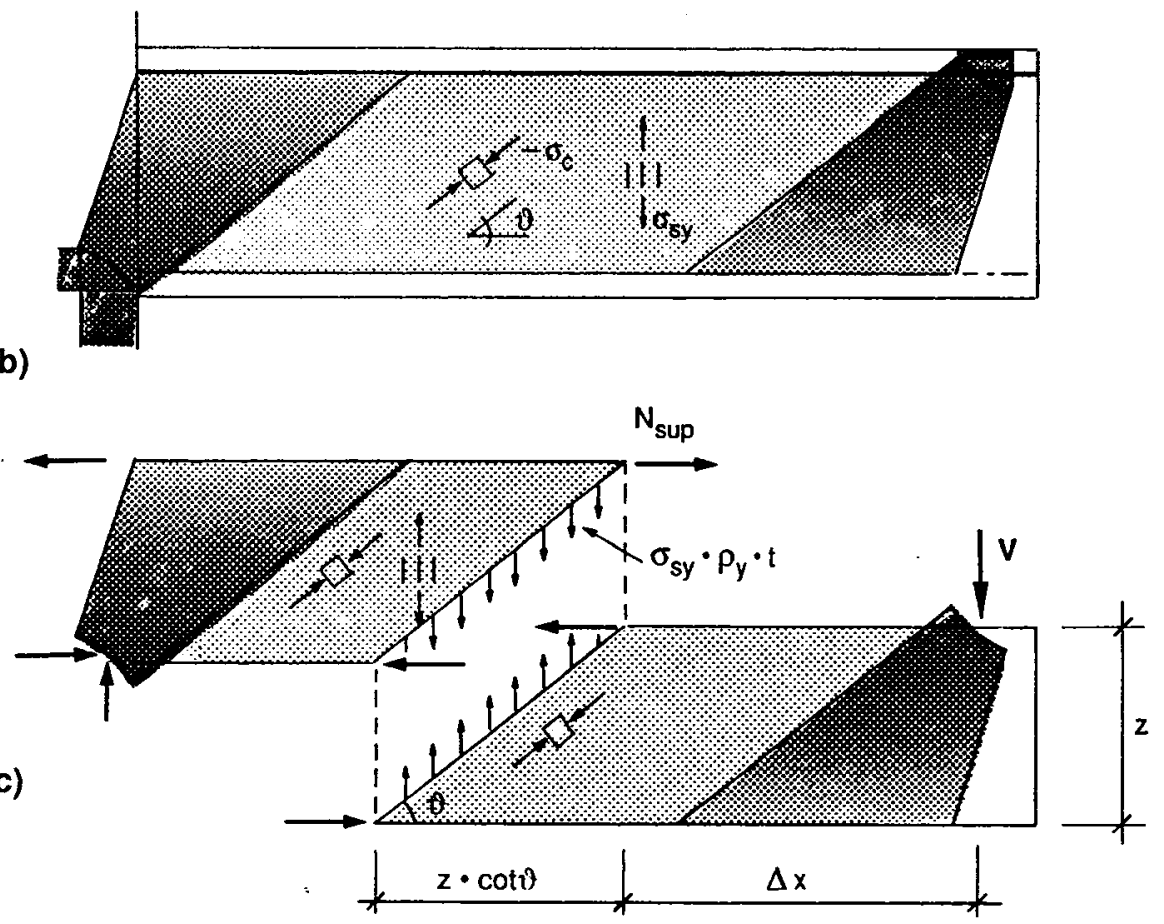

Bild 4.20: (a) Schubbeanspruchter Stahlbetonbalken; (b) Spannungsfeld für den Steg; (c) Schnitt für die Ermittlung der inneren Beanspruchungen.

Die Betonbeanspruchung im Steg beträgt (siehe Scheibenelement):

$$
\sigma_{C}=-\frac{\tau_{x y}}{\sin \vartheta \cdot \cos \vartheta} \quad \text { mit der Bezugsgrösse } \tau_{x y}=\frac{V}{t \cdot z}
$$

Bei stark bewehrten Trägern wird die effektive Festigkeit im Steg erreicht, bevor die Längs- und die Schubbewehrung geflossen sind. Dies erfolgt gemäss Plastizitätstheorie mit

$$
V_{R}=\frac{t \cdot z \cdot f}{2} c e \quad\left(\vartheta=45^{\circ}\right)
$$

Der zugehörige Mechanismus gemäss Plastizitätstheorie ist im Bild 4.21a dargestellt. 
(a)

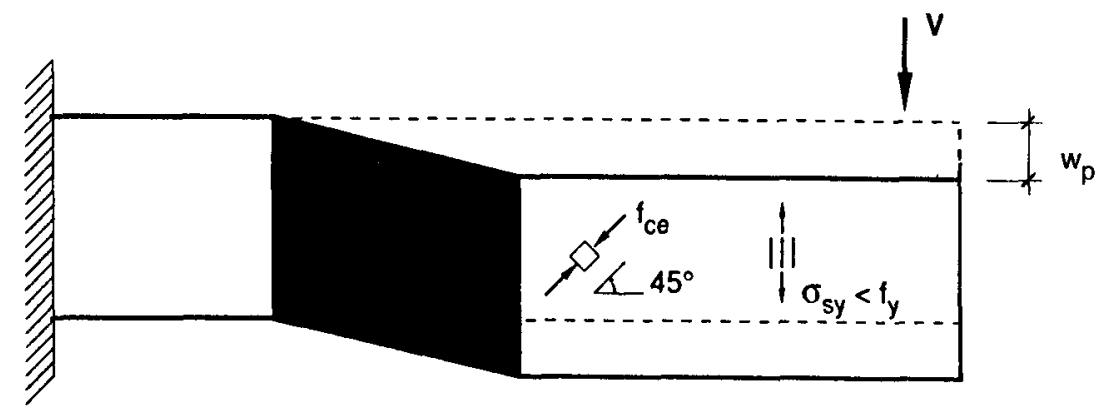

(b)

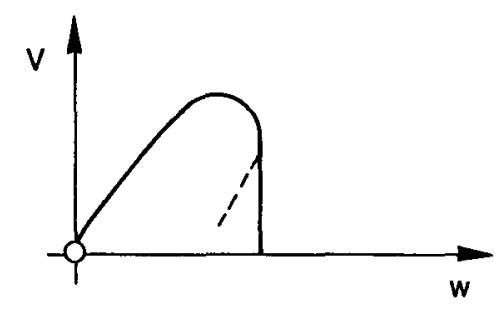

(c)

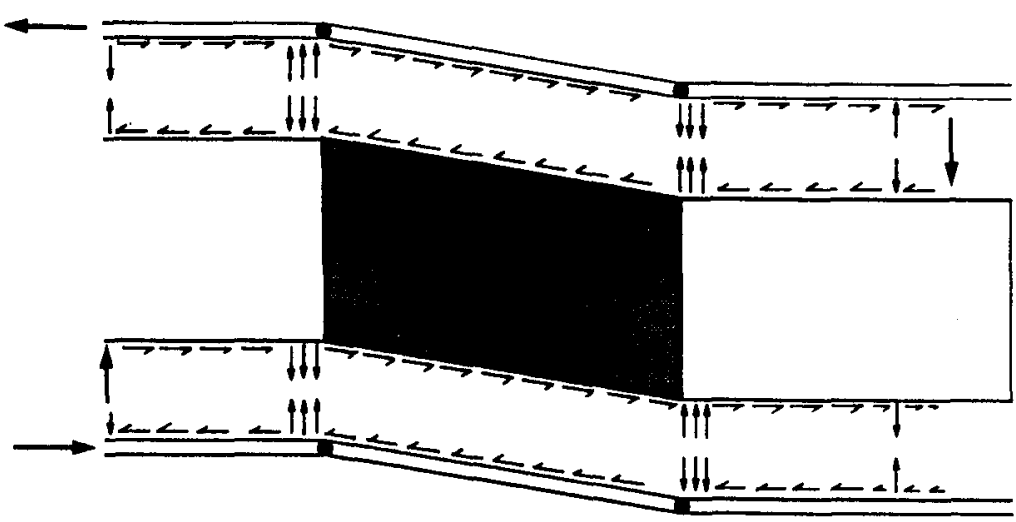

(d)

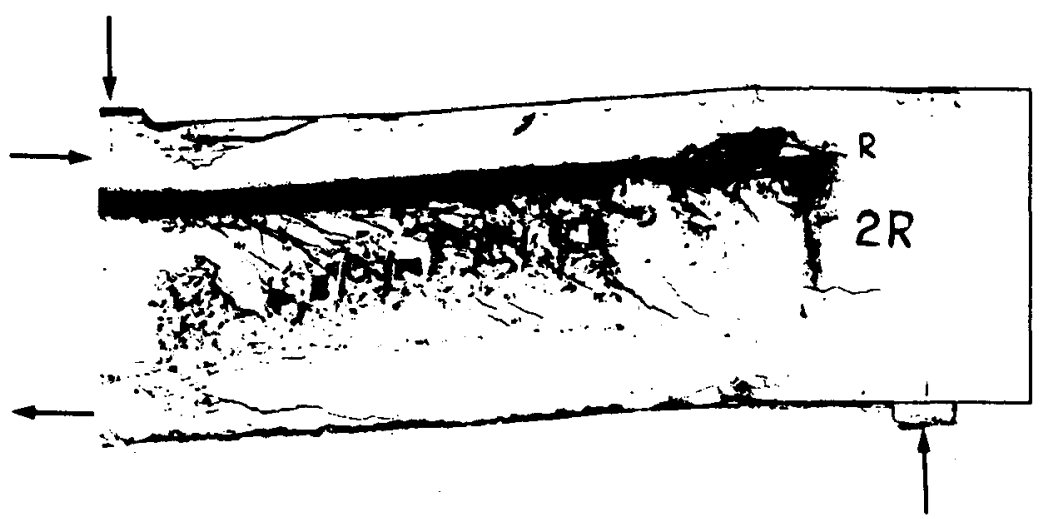

Bild 4.21: (a) Mechanismus nach Plastizitätstheorie für das Regime B (Betonbruch von Stahlfliessen); (b) qualitatives Last-Verformungs-Verhalten; (c) Mobilisierung Biegewiderstände der Gurten; (d) Bruchbild eines von Bennet und Balasooriya (1971) durchgeführten Versuchs (einfacher Balken). 
Wegen der Entfestigung des Betons nach dem Erreichen von ${ }^{\dagger} \mathrm{ce}$, findet wie beim Scheibenelement eine Verformungslokalisienung statt. Die dort dissipierbare Energie ist viel kleiner als die Energie, die im Balken gespeichert ist, so dass eine Entfestigungsinstabilităt gemăss Bild 4.21b erfolgt.

Wie aus Bild 4.21c ersichtlich, entsteht wegen der Schiebungsdifferenz zwischen Verformungsbereich und Entlastungsbereich ein Knick im Druck- und im Zuggurt. Dort wird demnach der Biegewiderstand aktiviert, so dass ein Teil der Querkraft im Verformungsbereich von den Gurten aufgenommen werden kann. Wenn der Biegewiderstand der Gurten genügend gross ist, kann eine Verformungslokalisierung verhindert werden. Das im Bild 4.21d dargestelte Bruchbild (Schubversuche von Bennet und Balasooriya (1971) zeigt einen solchen Fall. Es ist zu bemerken, dass der Widerstand der Guten bei der Ermittlung der Schubfestigkeit des Balkens im allgemeinen nicht berücksichtigt werden kann, weil dieser erst nach Erreichen der Stegfestigkeit aktiviert wird.

Bei kleinen Bewehrungsgehalten fliesst die Schubbewehrung, bevor der Beton bricht. Die Druckfeldneigung $\diamond$ kann aus der Gleichgewichtsbedingung

$$
\sigma_{s y} \cdot \rho_{y}=\tau_{x y} \cdot \tan \vartheta=\frac{V}{t \cdot z} \cdot \tan \vartheta \quad \text { mit } \sigma_{s y}=f y
$$

bestimmt werden:

$$
\tan \vartheta=\frac{f y \cdot \rho_{y}}{\tau_{x y}}=\frac{f_{y} \cdot \rho_{y} \cdot t \cdot z}{V}
$$

Bei Erhöhung der Beanspruchung $\tau_{x y}$ nimmt die Neigung $\vartheta$ ab, so dass die Betondruckspannung überproportional zunimmt:

$$
\sigma_{c}=-\frac{\tau_{x y}}{\sin \vartheta \cdot \cos \vartheta}=-\tau_{x y} \cdot(\tan \vartheta+\cot \vartheta)=-\left(t_{y} \cdot p_{y}+\frac{\tau_{x y}^{2}}{y \cdot \rho_{y}}\right)=-f_{c} \cdot\left[q y+\left(\frac{\tau_{y y}}{f_{c}}\right)^{2} \cdot \frac{1}{q_{y}}\right]
$$

Erreicht die Betondruckspannung die effektive Festigkeit $f_{c e}$ ' bricht der Beton (Regime BS). Wie beim Scheibenelement betrăgt die Traglast

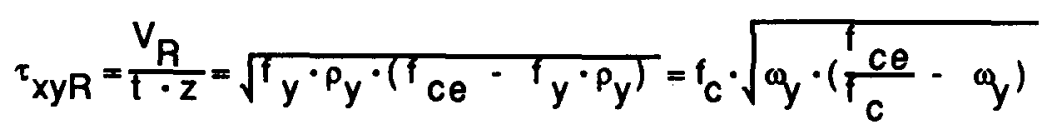

Der Mechanismus nach Plastizitătstheorie ist im Bild $4.22 \mathrm{a}$ dargestellt. Bild $4.22 \mathrm{~b}$ zeigt einen qualitativen Verlauf der Last-Vertormungs-Charakteristik. Typisch ist die Entfestigungsinstabilităt, die nach dem Betonbruch eintritt. Die Traglast ist im Bild 4.22c als Funktion des Bügelbewehrungsgehaltes graphisch ausgewertet. Im gleichen Bild sind auch die Resultate der im Anhang A1 ausgewerteten Balkenversuche eingetragen. Die Uebereinstimmung der Versuche mit den theoretischen Ueberlegungen ist befriedigend. Da die effektive Festigkeit konservativ festgelegt wurde (Bild 3.13c), ergeben fast alle Versuche eine höhere Traglast als die Theorie. 
(a)
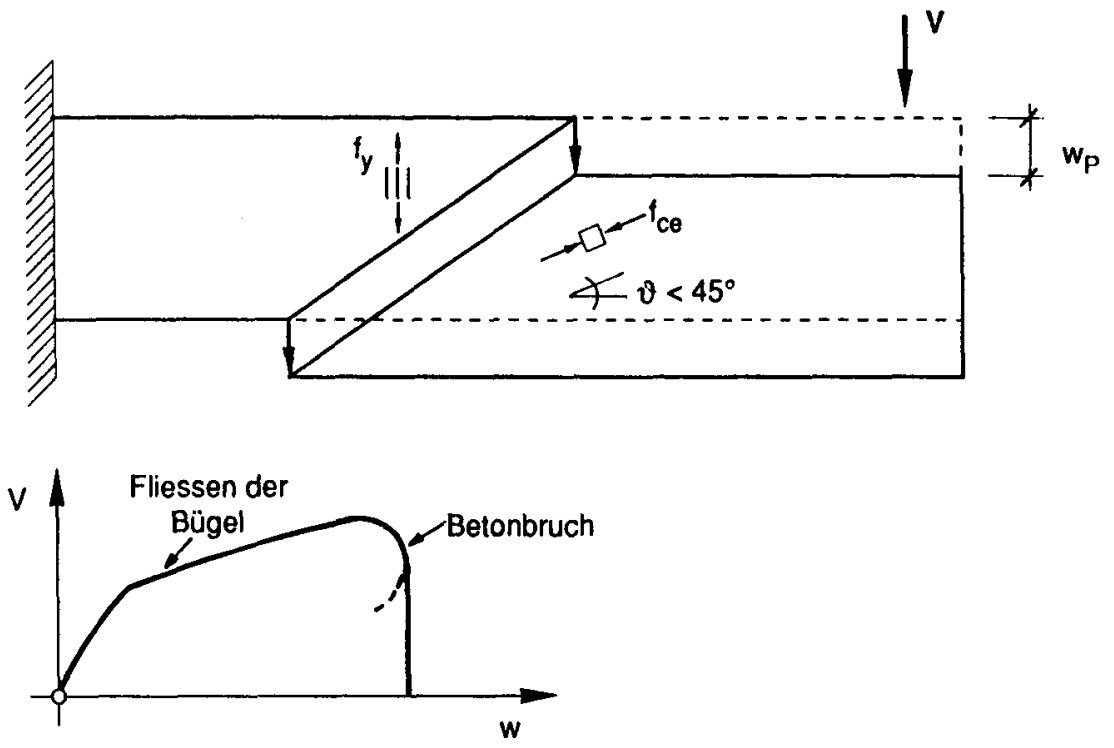

(b)

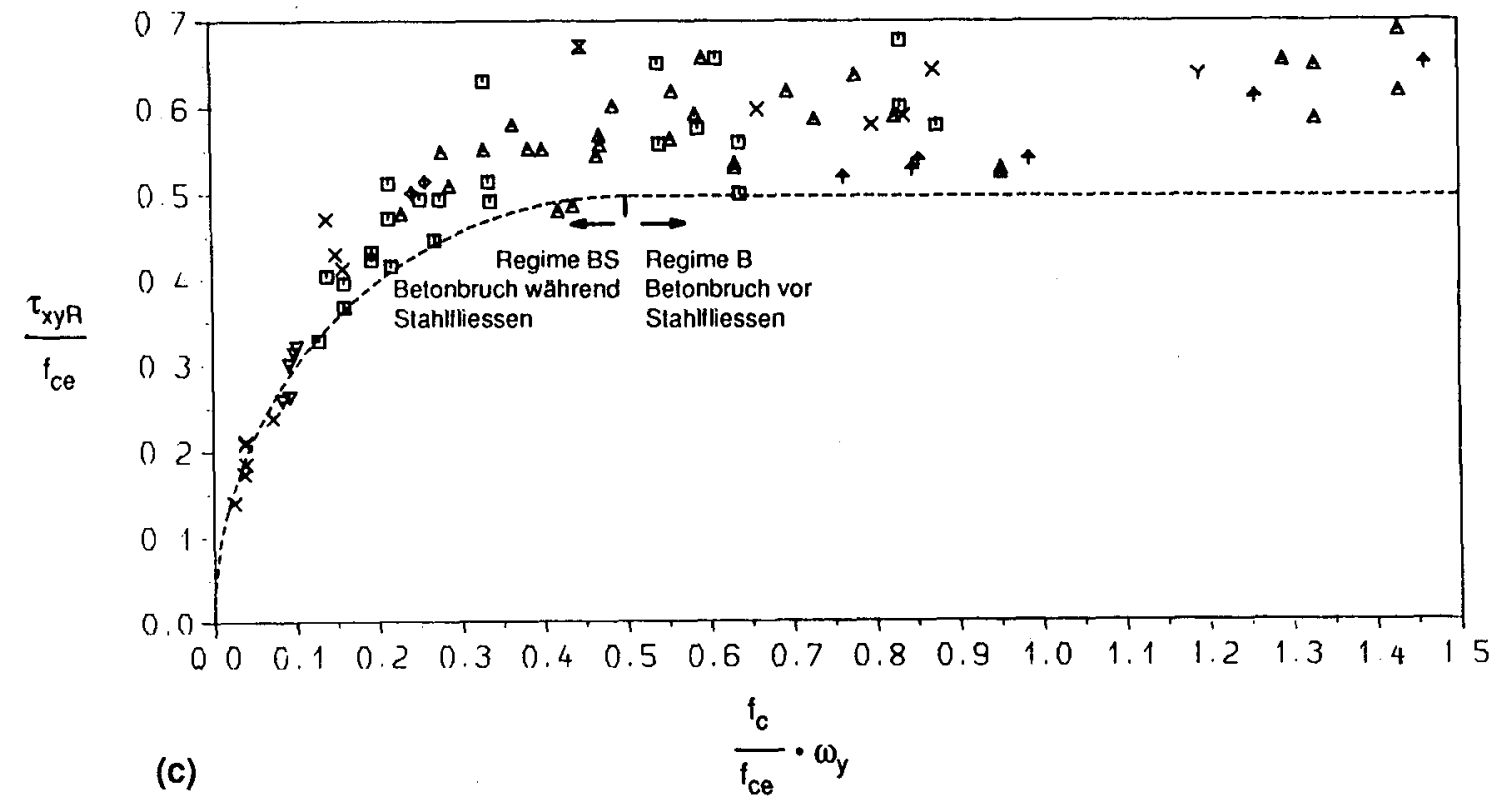

Bild 4.22: (a) Mechanismus nach Plastizitätstheorie für das Regime BS (Betonbruch wăhrend Stahlfliessen); (b) qualitatives Last-Verformungs-Verhalten; (c) experimentell ermittelte Traglast verglichen mit der theoretisch ermittelten (Regimes B und BS).

Der Fall mit Fliessen der horizontalen Bewehrung (Lảngsbewehrung) im schubbeanspruchten Bereich und Betonbruch sind nur möglich, wenn die Bewehrungsfliesskraft in diesem Bereich variiert. Bild 4.23a zeigt einen Fall mit abgestufter Bewehrung. Die Lăngsbewehrungsskraft setzt sich aus einem Anteil infolge von Biegung (gestrichelte Linie) und einem Anteil infolge von Querkraft zusammen. Da dieser vom Neigungswinkel des Druckfeldes abhăngig ist $\left(N_{\text {sup }}(V)=V / 2 \cdot \cot \vartheta\right)$, kann nach Erreichen der Fliesskraft im massgebenden Punkt die Last weiter erhöht werden, weil sich eine steilere Druckfeldneigung einstellen kann. Bild $4.23 \mathrm{~b}$ zeigt die Variation des Verlaufs von $\mathrm{V} / 2 \cdot \cot \vartheta$ wăhrend dieser Umlagerung. 
(a)

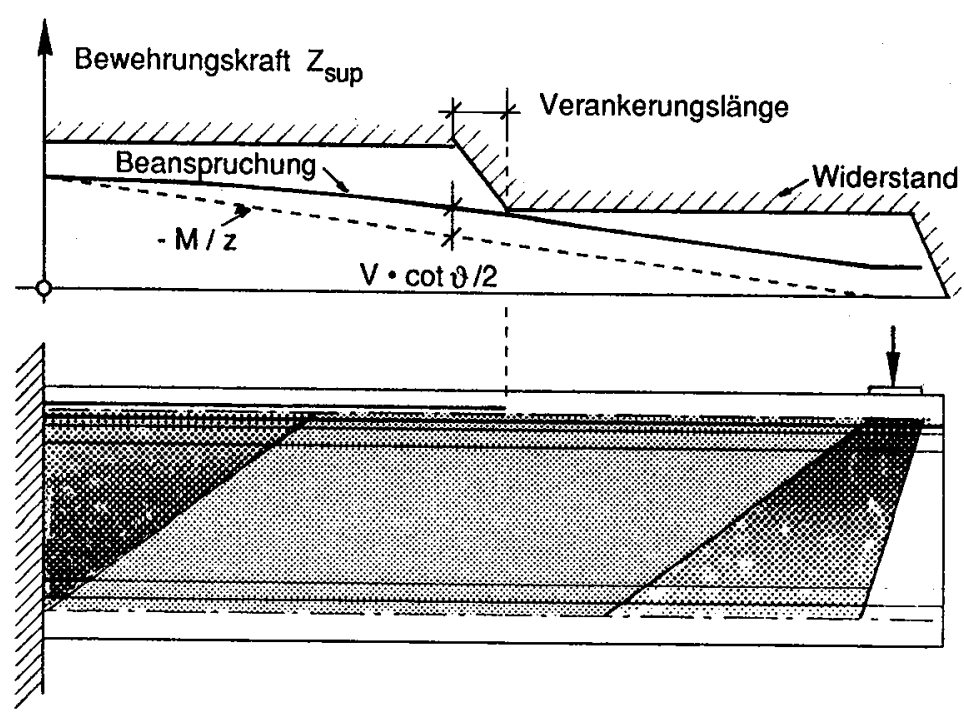

(b)

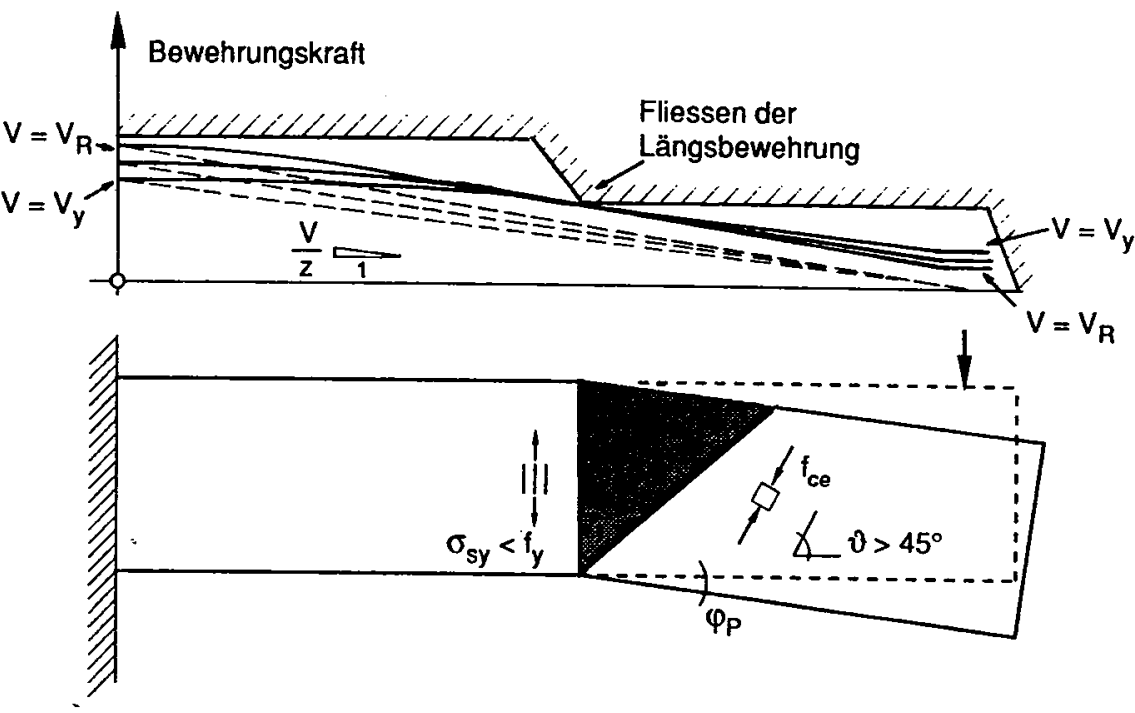

(d)

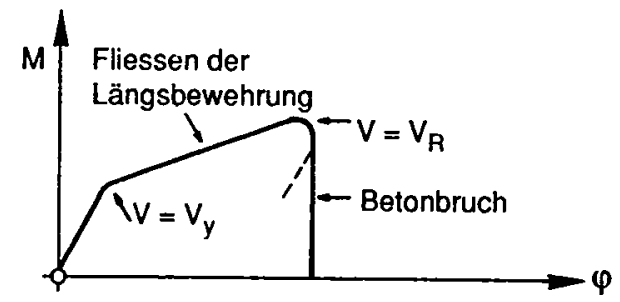

Bild 4.23: (a) Verlauf der Längsbewehrungskraft und Bewehrungswiderstand; (b) Umlagerung der inneren Kräfte; (c) Mechanismus nach Plastizitătstheorie für das Regime BS (Betonbruch während Fliessen der Längsbewehrung); (d) qualitatives Last-Verformungs-Verhalten. 
Wird während dieses Vorgangs die Betonfestigkeit im Steg erreicht, kann die Last nicht weiter gesteigert werden (Mechanismus nach Plastizitătstheorie im Bild 4.23c). Auch beim Regime BS verursacht im allgemeinen die Betonentfestigung im Steg eine Entfestigungsinstabilităt gemäss Bild 4.23d. Ist hingegen die Betonbeanspruchung gering, nimmt die Druckfeldneigung zu, bis auch die Bügelbewehrung fliesst (Regime S). Ein solcher Zustand, d.h. Fliessen beider Bewehrungen, kann auch erreicht werden, nachdem die Bügelbewehrung zuerst geflossen ist. In diesem Fall nimmt nach dem Erreichen der Streckgrenze die Druckfeldneigung in den Bügeln ab, bis auch die Längsbewehrung fliesst. Bild 4.24 zeigt den zugehörigen Mechanismus und zwei mögliche qualitative LastVerformungs-Verhalten (für nicht zu grosse Längsbewehrungsgehalte). Anhand des Vergleichs dieser Last-Verformungs-Beziehungen mit denjenigen der anderen Regimes ist klar ersichtlich, dass bei der Bemessung die letztere Bruchart anzustreben ist. Dies wird vor allem durch eine geeignete Wahl der Stegdicke erzielt.

(a)

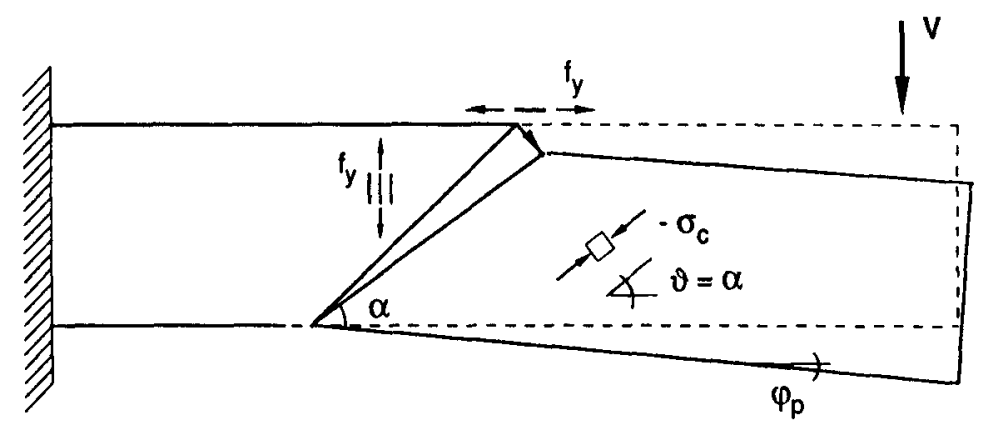

(b)
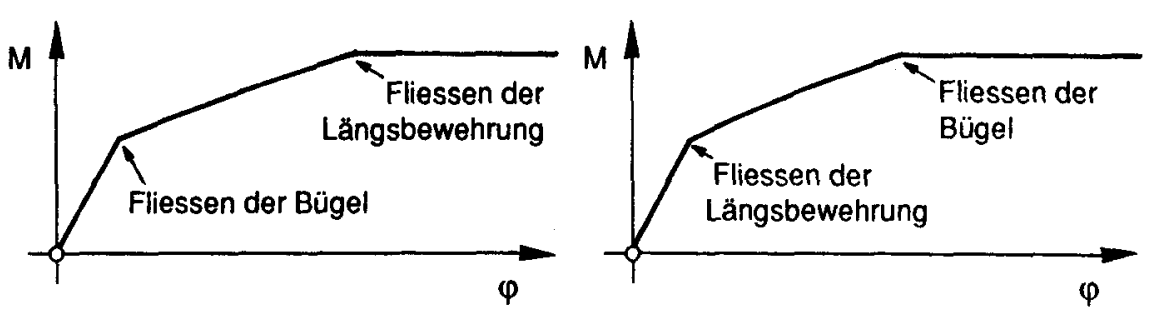

Bild 4.24: (a) Mechanismus nach Plastizitătstheorie für das Regime S; (b) mögliche Momenten-Rotations-Beziehungen. 


\section{Statisch unbestimmte Systeme mit Entfestigung}

Die Traglast von Systemen, deren sămtliche Elemente ein ideal plastisches Verhalten aufweisen, kann mit den Methoden der Plastizitătstheorie ermittelt werden. Diese Methoden basieren auf den im Abschnitt 2.2 enläuterten zwei Grenzwertsătze. Aus der Untersuchung des Verhaltens von Stahlbeton (Kapitel 3 und 4) geht hervor, dass die Last-Verformungs-Charakteristik vor allem dann entfestigend ist, wenn ein Betonversagen erfolgt. Diese Abweichung vom ideal plastischen Verhalten kann bei statisch unbestimmten Tragwerken zu einer Reduktion der Traglast führen. Dieser Vorgang wird zuerst anhand eines einfachen Modelis erläutert. Für einige übliche Tragwerksarten wird dann die Traglastreduktion infolge der Entfestigung quantifiziert und das Systemverhalten untersucht. Dabei werden die in den Kapiteln 3 und 4 angegebenen Last-Verformungs-Diagramme für Stahl, Beton und Stahlbeton durch bilineare Verläufe vereinfacht.

\subsection{Elnführendes Belsplel}

Das zu untersuchende Modell bestehend aus zwei parallel gekoppelten Stäben ist im Bild 5.1a dargestellt. Beide Stăbe verhalten sich bis zum Erreichen der Festigkeit linear elastisch. Danach lokalisiert sich die Verformung und die Festigkeit nimmt mit der Zunahme der Stabverlăngerung ab. Diese Phase endet mit dem totalen Verlust der Festigkeit. Im Abschnitt 2.3 wurden die Last-Verformungs-Diagramme eingehend diskutiert. Die Beziehungen lauten:

$$
\begin{array}{ll}
\text { im elastischen Bereich } & N_{i}=\frac{E A_{i}}{l} \cdot\left(u-u_{z, i}\right) ; \quad i=1,2 \\
\text { im plastischen Bereich } \quad N_{i}=\frac{N_{R, i}-D A_{i} \cdot\left(u-u_{z 1}\right)}{1-x_{i, i}} \\
\text { mit dem Entfestigungsstabilitătsfaktor } \quad x_{i, i}=\ell \cdot \frac{D A_{i}}{E A_{i}}
\end{array}
$$

Der erste Indizes des Entfestigungsstabilitătsfaktors besagt, in welchem Stab die Energiedissipation erfolgt, der zweite Indizes zeigt an, wo die elastische Energie gespeichert ist.

Im System ist schon vor der Belastung eine Zwängung vorhanden, die mit $u_{z 1}$ und $u_{z 2}$ beschrieben ist. Da in diesem Zustand $(\mathrm{N}=0)$ auch die Verlăngerung des Systems null ist, gilt:

$$
u_{z 1} \cdot E A_{1}=-u_{z 2} \cdot E A_{2}
$$

Bild 5.1b zeigt die Last-Verformungs-Kurven für beide Stäbe und die gleichen Kurven für das System. Bild 5.1c zeigt die Last-Verformungs-Kurven des Systems für verschiedene Werte von DA und $\mathrm{DA}_{2}$. Die Kurven kőnnen aus der einfachen Superposition der Stabcharakteristiken gewonnen werden. Wenn sich beide Stâbe elastisch verhalten, gilt

$$
N=u \cdot \frac{E A_{1}+E A_{2}}{\ell}
$$


(a)
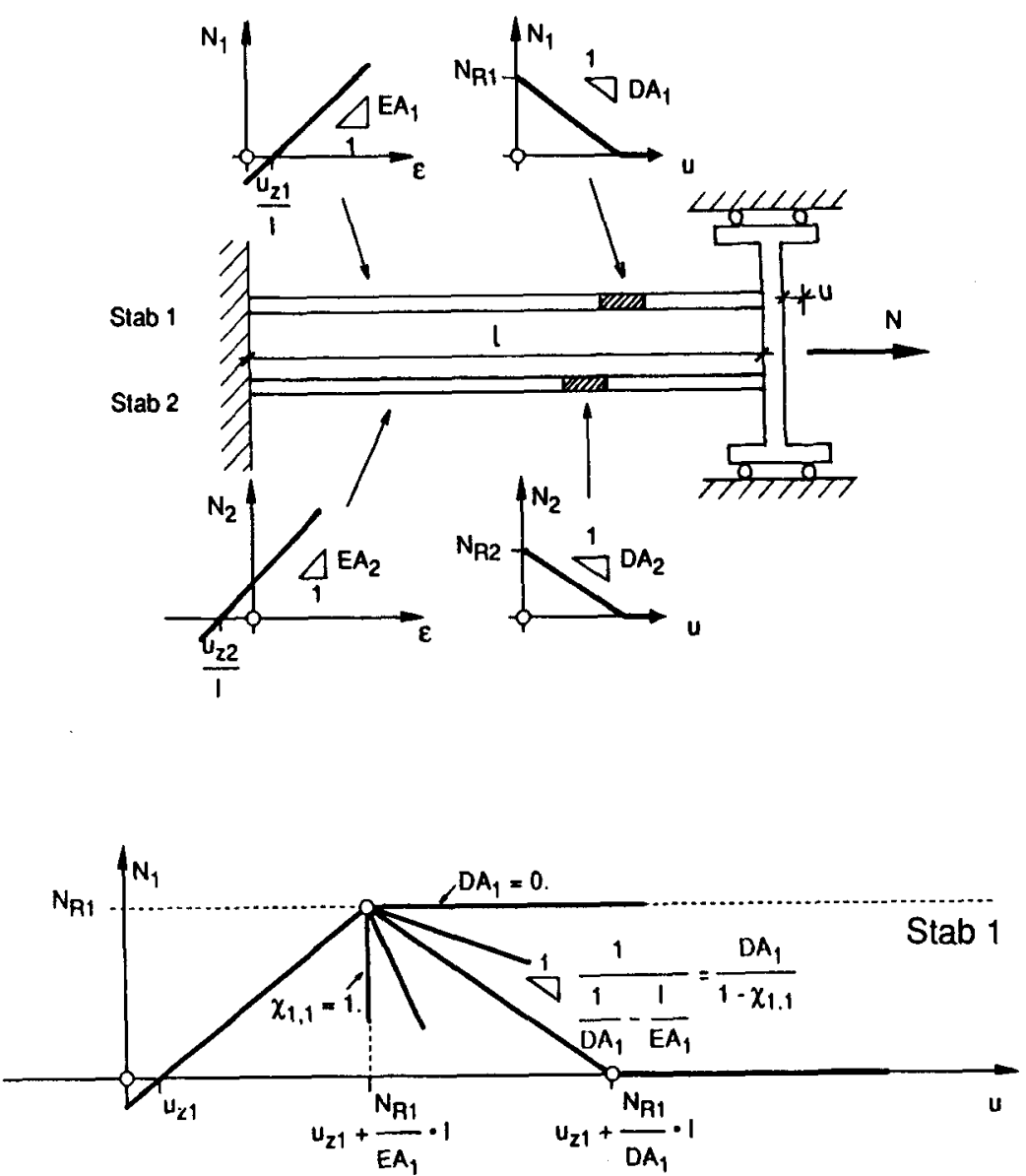

(b)

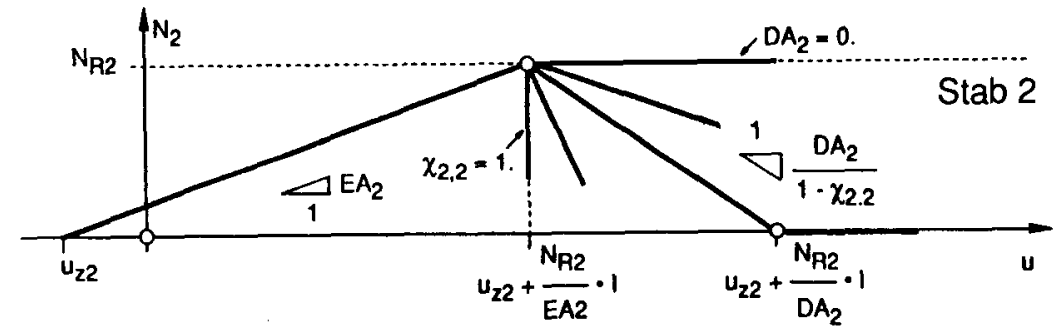

(c)

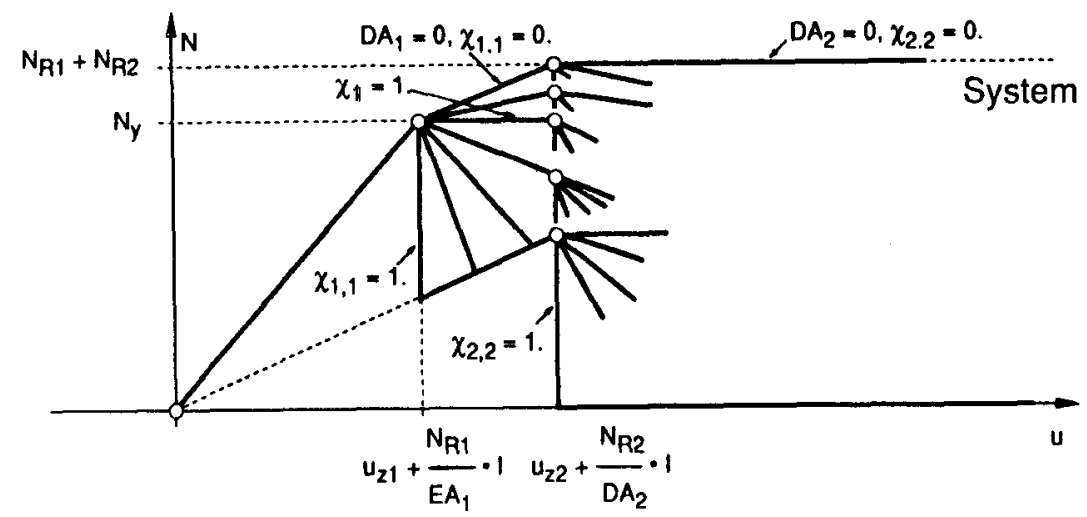

Bild 5.1: Einfach statisch unbestimmtes System; Last-Verformungs-Diagramm der Stäbe und des Systems. 
Nimmt man an, dass der Stab 1 zuerst fliesst, so tritt dies ein für

$$
u=u_{z 1}+\frac{N_{R 1}}{E A_{1}} \cdot l
$$

mit der Belastung

$$
N=N_{y}=\left(\frac{u_{21}}{l} \cdot E A_{1}+N_{R 1}\right) \cdot\left(1+\frac{E A_{2}}{E A_{1}}\right)
$$

Danach folgt die elastisch-plastische Phase:

$$
N=u \cdot\left(-\frac{D A_{1}}{1-x_{1,1}}+\frac{E A_{2}}{l}\right)+\frac{N_{R 1}}{1-x_{1,1}}+\frac{u_{z 1}}{l} \cdot \frac{E A_{1}}{1-x_{1,1}}
$$

Diese Phase endet entweder mit der Plastifizierung des zweiten Stabes bei der Verlăngerung

$$
u=u_{z 2}+\frac{N_{R 2}}{E A_{2}} \cdot 1
$$

oder mit der Erschöpfung des Widerstandes des ersten Stabes bei der Verlăngerung

$$
u=u_{z 1}+\frac{N_{R 1}}{D A_{1}}
$$

Im zweiten Fall kann die Last wiederum erhőht werden bis zum Erreichen der Festigkeit des zweiten Stabes.

Die Systemsteifigkeit in der elastisch-plastischen Phase

$$
\frac{d N}{d u}=-\frac{D A_{1}}{1 \cdot x_{1,1}}+\frac{E A_{2}}{l}
$$

liegt zwischen - $\infty$ (Entfestigungsinstabilität im Stab 1 mit $x_{1,1}=1$ ) und $E A_{2} / \ell$ (ideal plastisches Verhalten des Stabes 1 mit $\mathrm{DA}_{1}=0$ ). Die Systemsteifigkeit wird gleich null für

$$
\frac{\mathrm{DA}_{1}}{1-x_{1,1}} \cdot \frac{l}{E A_{2}}=1
$$

In diesem Fall kompensiert die Zunahme der Beanspruchung im Stab 2 (elastisch) gerade die Abnahme der Festigkeit infolge Entfestigung im Stab 1. Eine solche Umlagerung der innneren Kräfte erfolgt mit einer Zunahme der Verlăngerung und mit einer konstanten Belastung, so dass wăhrend dieses Vorgangs Energie dissipiert wird. Ein ăhnlicher Vorgang könnte jedoch auch ohne Last erfolgen, d.h. ohne Energiedissipation. Dies ist beispielsweise der Fall, wenn in einem Stab die Festigkeit infolge innerer Zwăngung und ohne ăussere Last erreicht wird. Mit

$$
\frac{\mathrm{DA}_{1}}{1-x_{1,1}} \cdot \frac{l}{E A_{2}}=1 \text { oder } \frac{\mathrm{DA}_{2}}{1-x_{2,2}} \cdot \frac{l}{E A_{1}}=1
$$


erfolgt eine Entfestigungsinstabilität, bei der die im System (Stäbe 1 und 2) elastisch gespeicherte Energie voll im Verformungsbereich eines Stabes dissipiert wird.

Folgende Entfestigungsstabilitătsfaktoren können eingeführt werden:

$$
x_{1}=\frac{\mathrm{DA}_{1}}{1-x_{1.1}} \cdot \frac{l}{E A_{2}}=1 \quad \text { und } \quad x_{2}=\frac{\mathrm{DA}_{2}}{1-x_{2.2}} \cdot \frac{l}{E A_{1}}=1
$$

Diese Entfestigungsstabilitătsfaktoren können mit einem Belastungssystem wie im Bild 5.2a dargestellt berechnet werden und beziehen sich auf das ganze System. Sie sind nicht zu verwechseln mit $x_{1,1}$ und $x_{2,2}$, die sich auf die isolierten Stäbe beziehen.

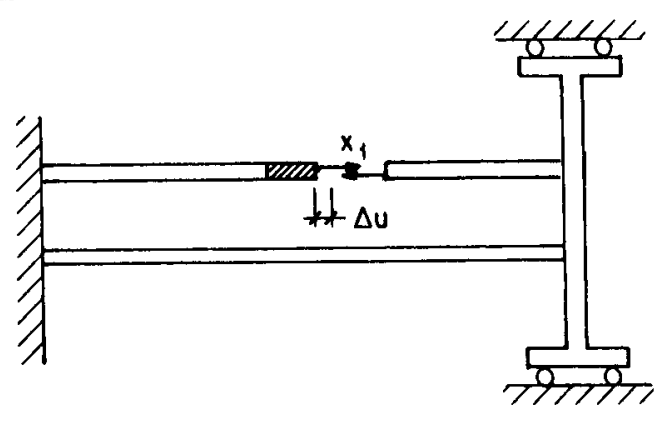

(a)

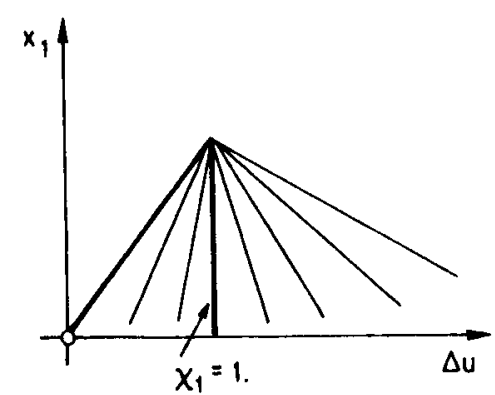

(b)

Bild 5.2: Entfestigungsinstabilităt eines statisch unbestimmten Tragwerks

Die Untersuchung des Entfestigungstabilitătsfaktors für das ganze System ist vor allem bei der Analyse von mehrfach statisch unbestimmten Systemen interessant. Zur Bestimmung der Traglast kann die Bildung plastischer Gelenke schrittweise verfolgt werden solange der Entfestigungsstabilitätsfaktor kleiner als 1 ist. Uebersteigt dieser den Wert 1, wird die Last-Verformungs-Charakteristik des Systems entfestigend, d.h., die Traglast ist bereits erreicht worden. Der Entfestigungsstabilitätsfaktor lăsst sich ermitteln, indem bei jeder Bildung plastischer Gelenke dort eine Bindung gelöst und eine Belastung (Kraft oder Moment) eingeführt wird. Dabei ist zu berücksichtigen, dass sich bereits ausgebildete plastische Gelenke nicht mehr elastisch, sondern plastisch oder entfestigend verhalten.

Eine ăhnliche Methode ist von Darvall (1983) für Durchlauftrăger und Rahmen vorgeschlagen worden. 


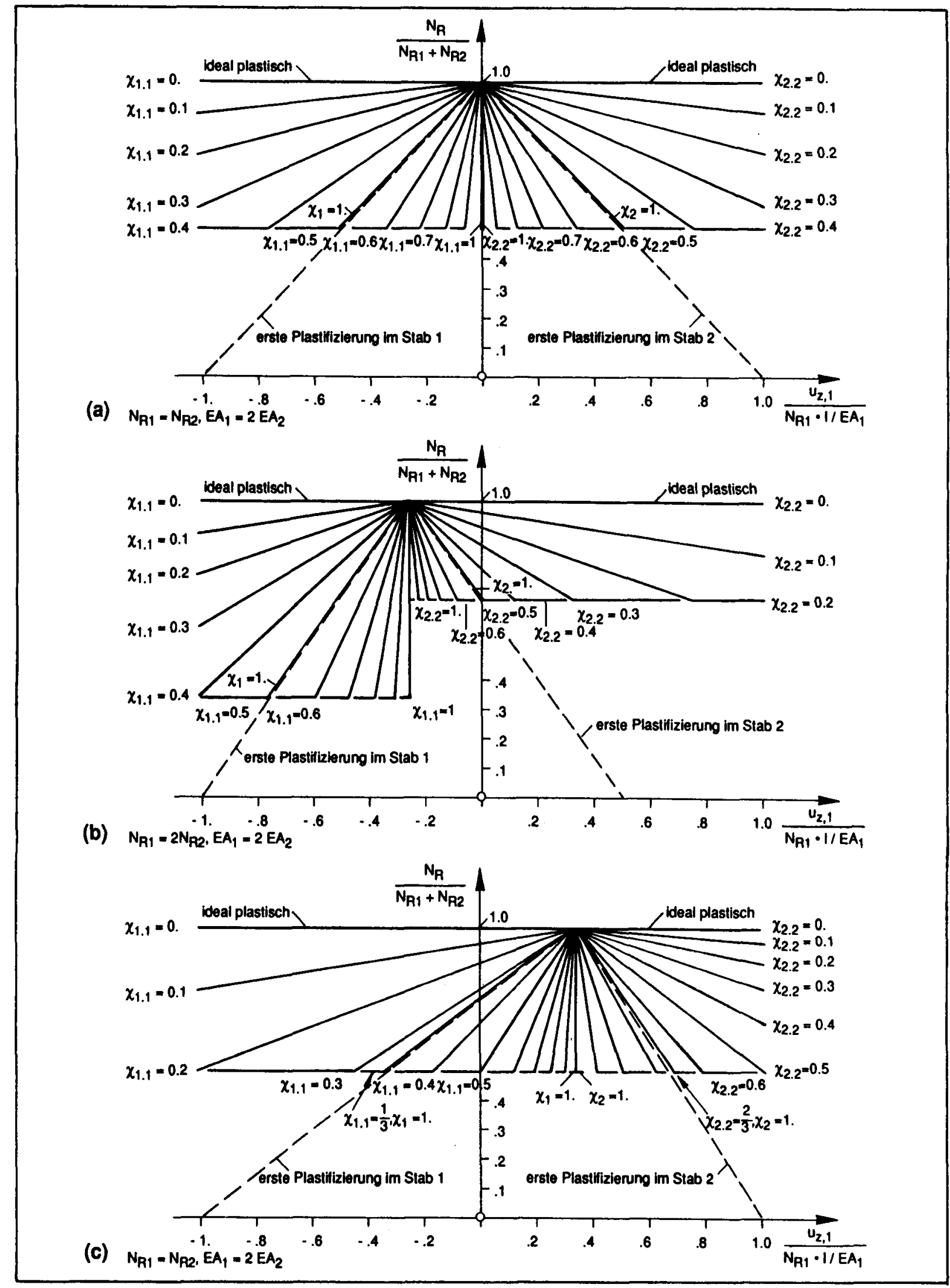

Bild 5.3: Traglast als Funktion der Zwăngung für verschiedene Entfestigungsstabilitătsfaktoren und verschiedene Parameterkombinationen 
Im Bild 5.3 ist der Einfluss der Zwăngung und des Entfestigungsstabilitătsfaktors auf die Last, bei welcher die erste Plastifizierung (gestrichelte Linie) und auf die Last, bei welcher die zweite Plastifizierung (ausgezogene Linie) erfolgt, für verschiedene Parameterkombinationen graphisch dargestellt. Auch aus diesen Diagrammen geht hervor, dass für $\chi_{1} \geq 1$ und $\chi_{2} \geq 1$ die Traglast bereits bei der ersten Plastifizierung erreicht wird.

Weiter ist zu bemerken, dass die Traglast nur von der Entfestigungs-Charakteristik des zuerst plastifizierten Stabes beeinflusst wird. Damit in einem statisch unbestimmten System die Widerstände einzelner Elemente im Bruchzustand möglichst ausgenützt werden, ist das System so zu entwerfen, dass die duktilen Elemente möglichst frühzeitig plastifiziert und die spröden Elemente möglichst spät aktiviert werden. Sind mehrere spröde Elemente vorhanden, dann ist eine möglichst gleichzeitige Aktivierung der Widerstände dieser Elemente vorteilhaft. Die Traglast derart bemessener Tragwerke kann trotz Entfestigung mit der Plastizitătstheorie ermittelt werden. Diese Zusammenhănge zeigt Bild 5.4.
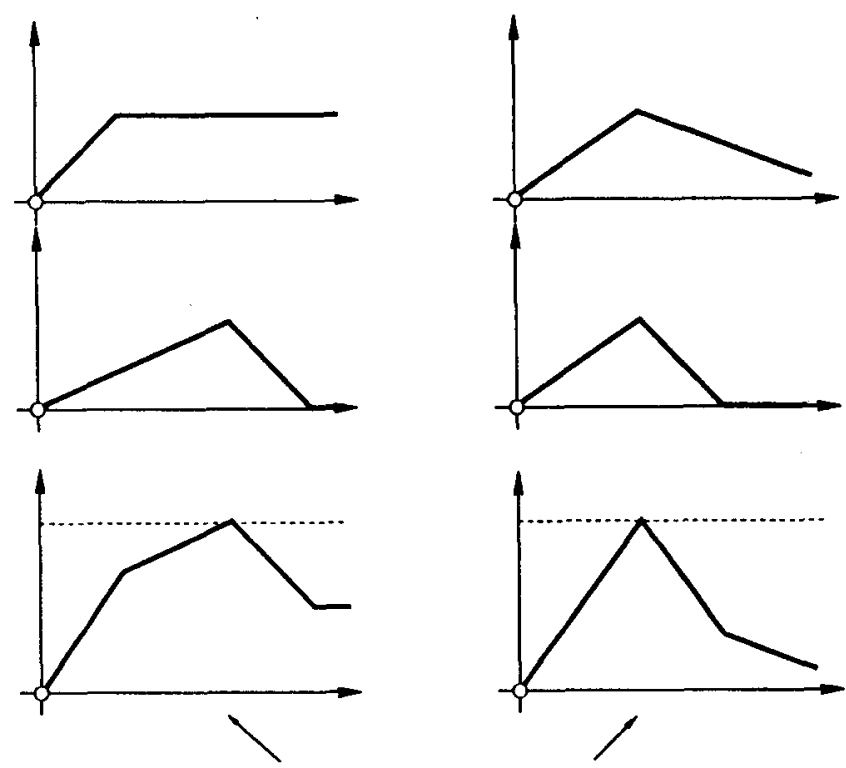

Mit der Plastizitătstheorie wird die Traglast richtig abgeschätzt
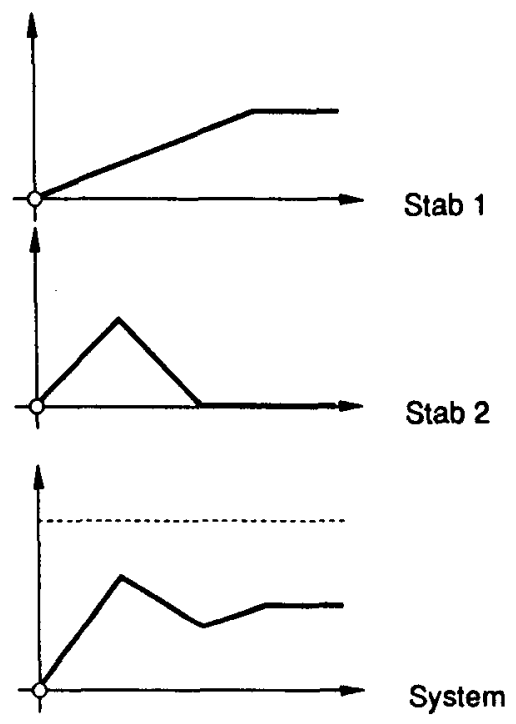

Die Anwendung der Plastizitătstheorie führt zu einer Ueberschätzung der Traglast

Bild 5.4: Einfluss der Entfestigung auf die Traglast 


\subsection{Systeme mit plastischen Verformungen in den Blegegelenken}

Als Beispiel wird der im Bild 5.5 dargestellte einfach statisch unbestimmte Trăger untersucht.

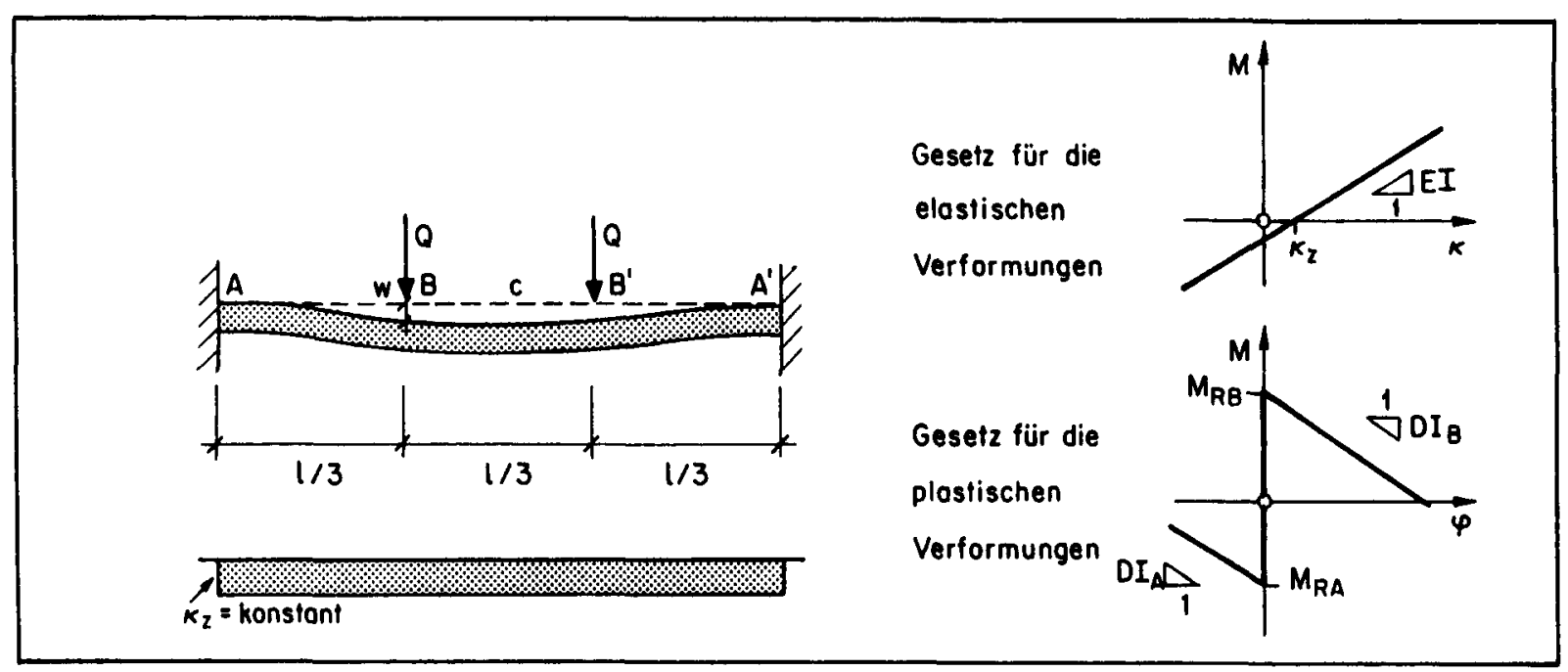

Bild 5.5: System und Materialverhalten

Ein ähnliches System mit dem gleichen Materialverhalten ist von Maier (1968) behandelt worden. Folgende dimensionslosen Normierungsgrössen werden eingeführt:

- Für die Last: $\quad \eta=\frac{Q}{Q_{R, p l}}$ wobei $Q_{R, p l}=$ Traglast eines ideal plastischen Systems $\quad Q_{R, p l}=\frac{3}{l} \cdot\left(-M_{R, A}+M_{R, B}\right)$

- Für die Durchbiegung: $\quad \zeta=\frac{w}{w_{R, \text { el }}}$ wobei $w_{R, \text { el }}=$ elastisch berechnete Durchbiegung infolge $Q_{R, p l}: w_{R, e l}=\frac{Q_{R, p l} \cdot l^{3}}{162 \cdot E l}$

- Für die Zwängung: $\quad \psi=\frac{\kappa_{z} \cdot E I}{-M_{R A}+M_{R B}}$

$\kappa_{z}$ ist eine über die Balkenlänge konstante Krümmung, die vorhanden ist, wenn der Trăger unbelastet ist. Sie wird hier stellvertretend als Zwångung berücksichtigt.

- Für die Momente:

$$
\mu_{A}=\frac{M_{A}}{-M_{R A}+M_{R B}} ; \mu_{B}=\frac{M_{B}}{-M_{R A}+M_{R B}}
$$

- Für die Widerstănde: $\quad \mu_{\mathrm{RA}}=\frac{M_{\mathrm{RA}}}{-M_{\mathrm{RA}}+M_{\mathrm{RB}}}{ }^{\mathrm{RB}}=\frac{M_{\mathrm{RB}}}{-M_{\mathrm{RA}}+M_{\mathrm{RB}}} ;-\mu_{\mathrm{RA}}+\mu_{\mathrm{RB}}=1$

Da das System nicht wie im vorangehenden Beispiel in zwei unabhăngige Subsysteme aufgeteilt werden kann, ist es nicht möglich, die Systemcharakteristik aus einer einfachen Superposition der Eigenschaften der Subsysteme zu erhalten. Dieses Problem kann mit den üblichen Methoden der Baustatik gelöst werden. 
Im elastischen Zustand gilt:

$$
W=\frac{Q \cdot l^{3}}{162 \cdot E T} ; \quad M_{A}=-\frac{2}{9} \cdot Q \cdot l-\kappa_{Z} \cdot E I ; \quad M_{B}=\frac{1}{9} \cdot Q \cdot l-\kappa_{Z} \cdot E I
$$

und mit den dimensionslosen Grössen:

$$
\eta=\zeta ; \quad \mu_{A}=-\frac{2}{3} \cdot \eta-\psi ; \quad \mu_{B}=\frac{1}{3} \cdot \eta-\psi
$$

Je nach dem Verhältnis der Widerstănde $-\mu_{R A} / \mu_{R B}$ und der Zwängung $\psi$ erfolgt die Plastifizierung zuerst entweder in einer der Einspannstellen ( $A$ oder $A^{\prime}$ ) oder im mittleren Bereich, d.h. zwischen $B$ und $B^{\prime}$.

Der erste Fall findet für $\mu_{A}=\mu_{R A}$, d.h. $\eta=\zeta=\frac{3}{2} \cdot\left(-\mu_{R A}-\psi\right)$, statt. Wie Bild 5.6 zeigt, verursacht eine plastische Rotation $\delta \varphi_{A}$ in $A$ eine Momentenzunahme $\delta M_{A}$ infolge Entfestigung $\left(M_{A}\right.$ ist negativ), die nur mit einer Beanspruchungszunahme in $A^{\prime}$ vertrăglich sein kann. Dies ist jedoch nicht zulăssig, da auch in $A^{\prime}$ die Festigkeit erreicht wurde. Das bedeutet, dass auch in $A^{\prime}$ eine plastische Verformung erfolgen muss, damit das System noch symmetrisch bleibt.

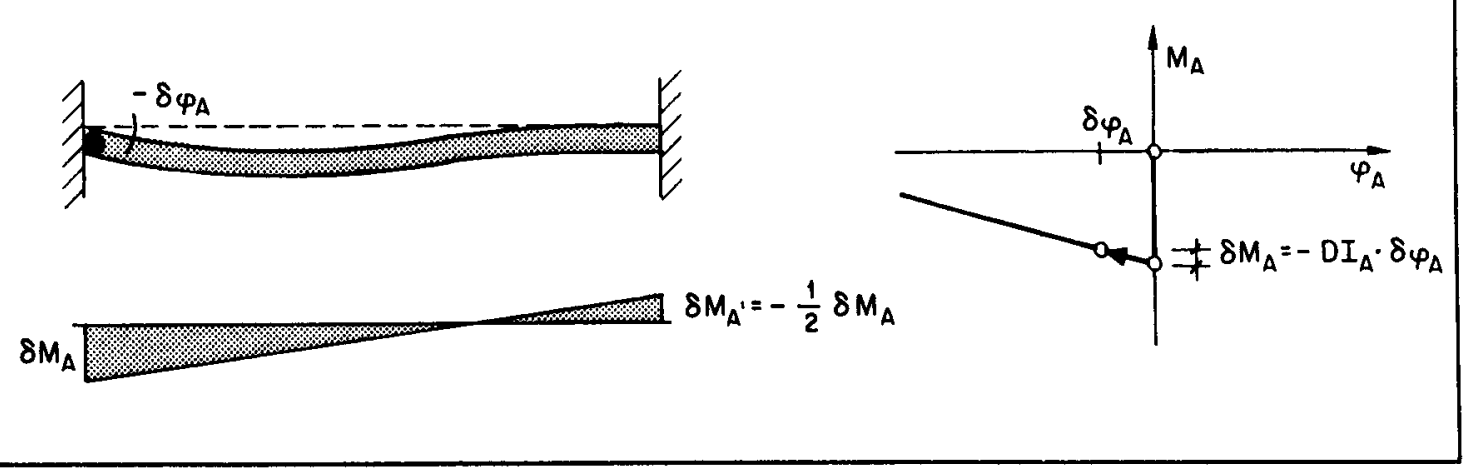

Bild 5.6: Beanspruchungsinkrement infolge plastischer Verformung in $\mathrm{A}$

Der Entfestigungsstabilitătsfaktor lässt sich ermitteln, indem Gelenke mit den zugehörigen Beanspruchungen in $A$ und in $A^{\prime}$ eingeführt werden (Bild 5.7a). In jedem Gelenk wird die elastisch gespeicherte Energie der zugehörigen Balkenhălfte dissipiert, so dass der Entfestigungsstabilitătsfaktor

$$
x_{A}=\frac{\ell}{2} \cdot \frac{D I}{E T}
$$

ist.

In diesem elastisch-plastischen Zustand gilt:

$$
\begin{aligned}
& \eta=\frac{1-x_{A}}{5-x_{A}} \cdot \zeta \cdot 6 \cdot \frac{\mu_{R A}+\psi}{5-x_{A}} \\
& \mu_{A}=\frac{\mu_{R A}+x_{A} \cdot \psi+\frac{2}{3} \cdot x_{A} \cdot \eta}{1-x_{A}} ; \mu_{B}=\frac{\mu_{R A}+x_{A} \cdot \psi+\left(1-\frac{1}{3} \cdot x_{A}\right) \cdot \eta}{1-x_{A}}
\end{aligned}
$$



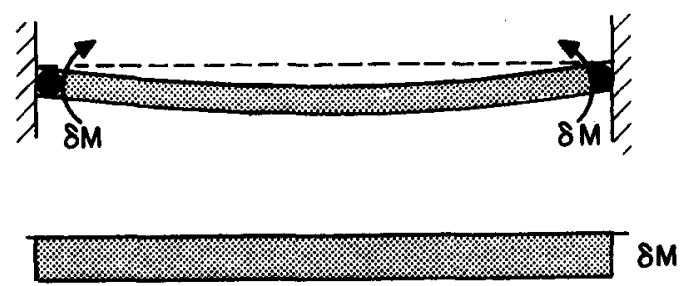

(a) Plostische Gelenke in den Einsponnstellen
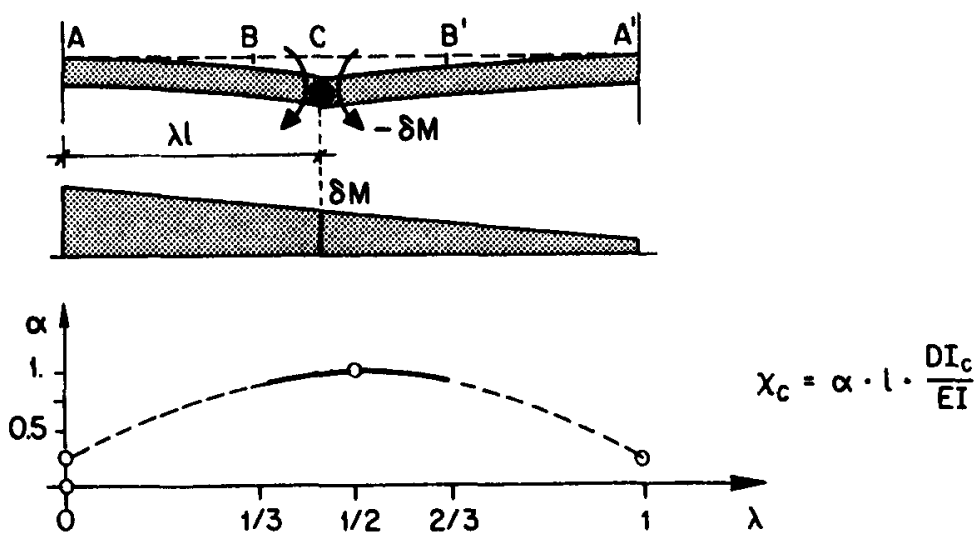

(b) Plastisches Gelenk im mittleren Bereich

Bild 5.7: Beanspruchungsänderung infolge von Entfestigung

Die Grenze dieses Regimes wird erreicht, wenn ein drittes plastisches Gelenk zwischen B und B' entsteht. Mit

$$
\mu_{B}=\mu_{R B}
$$

gilt

$$
\eta=\frac{1-x_{A} \cdot\left(\mu_{\mathrm{RB}}+\psi\right)}{1-\frac{1}{3} \cdot x_{\mathrm{A}}}
$$

Bei grossen Widerstandsverhăltnissen $-\mu_{\mathrm{RA}} / \mu_{\mathrm{RB}}$ und grossen negativen Zwăngungen tritt die erste Plastifizierung im mittleren Bereich ein. Dies erfolgt für

$$
\mu_{B}=\mu_{R B} \text {, das heisst } \quad \eta=\zeta=3 \cdot\left(\mu_{\mathrm{RB}}+\psi\right)
$$

Obwohl die Beanspruchung im Bereich B-B' konstant ist, erfolgt in einem kleinen Bereich wegen der Entfestigung eine Lokalisierung der Verformungen. Die Entfestigung in diesem Bereich verursacht im ganzen Bereich B-B' eine Abnahme der Beanspruchung, so dass eine derartige Verformung zulässig ist. Bild 5.7b zeigt die Ermittlung des Entfestigungstabilitătsfaktors. Dieser ist von der Lage des plastischen Gelenks abhăngig: 


$$
x_{C}=\alpha \cdot 1 \cdot \frac{D I C}{E T} \text { mit } \quad \alpha=1-\frac{3}{4} \cdot \frac{(1-2 \cdot \lambda)^{2}}{1-3 \cdot \lambda+3 \cdot \lambda^{2}}
$$

Die Last-Verformungs-Beziehung für diesen elastisch-plastischen Zustand lautet:

$$
\eta=\frac{1-x_{C}}{1+\alpha-x_{C}} \cdot \zeta+3 \cdot \alpha \cdot \frac{\mu_{\mathrm{RB}}+\psi}{1+\alpha-x_{\mathrm{C}}}
$$

Für $\lambda \neq 1 / 2$ ist das System unsymmetrisch geworden, und wie Bild $5.7 \mathrm{~b}$ zeigt, ist für $\lambda<1 / 2$ die Beanspruchung in $A$ grösser als in $A^{\prime}$, so dass die Festigkeit zuerst in $A$ erreicht wird. Nach der Bildung des zweiten Gelenkes in A muss die Verformung noch vergrössert werden, bis ein labiler Zustand mit einem dritten Gelenk in $A^{\prime}$ erreicht wird. Vereinfachend wird angenommen, dass sich die Verformungen des Gelenkes $C$ in Balkenmitte lokalisieren. Die Gleichungen für den Entfestigungsstabilitätsfaktor, die Last-Verformungs-Beziehung und die Beanspruchungen lauten:

$$
\begin{aligned}
& x_{C}=\ell \cdot \frac{D I C}{E T} \\
& \eta=\frac{1-x_{C}}{2-x_{C}} \cdot \zeta+3 \cdot \frac{\mu_{R B}+\psi}{2-x_{C}} \\
& \mu_{A}=\frac{\mu_{R B}+x_{C} \cdot \psi+\left(\frac{2}{3} \cdot x_{C}-1\right) \cdot \eta}{1-x_{C}} ; \mu_{B}=\frac{\mu_{R B}+x_{C} \cdot \psi-\frac{1}{3} \cdot x_{C} \cdot \eta}{1-x_{C}}
\end{aligned}
$$

Die Grenze des Regimes wird erreicht für

$$
\mu_{A}=\mu_{R A} \text {, das heisst für } \eta=\frac{1+x_{C} \cdot\left(\mu_{R A}+\psi\right)}{1-\frac{2}{3} \cdot x_{C}}
$$

Wird die Durchbiegung weiter vergrössert, entstehen plastische Gelenke auch bei den Einspannstellen. Die Last-Verformungs-Charakteristik und die Beanspruchungen in diesem Zustand sind mit folgenden Beziehungen beschrieben:

$$
\begin{aligned}
& \eta=\frac{x_{A}+x_{C}-x_{A} \cdot x_{C}}{2 \cdot x_{A}+5 \cdot x_{C}-x_{A} \cdot x_{C}-9 \cdot \zeta \cdot 3 \cdot \frac{\left(2 \cdot x_{C}-x_{A}\right) \cdot\left(\mu_{R A}+\psi\right)+3-x_{A}}{2 \cdot x_{A}+5 \cdot x_{C}-x_{A} \cdot x_{C}-9}} \\
& \mu_{A}=\frac{\mu_{R A} \cdot\left(x_{A}+x_{C}\right)+x_{A}+x_{A} \cdot x_{C} \cdot \psi+x_{A} \cdot\left(\frac{2}{3} \cdot x_{C}-1\right) \cdot \eta}{x_{A}+x_{C}-x_{A} \cdot x_{C}} \\
& \mu_{B}=\frac{\mu_{R A} \cdot\left(x_{A}+x_{C}\right)+x_{A}+x_{A} \cdot x_{C} \cdot \psi+x_{C} \cdot\left(1-\frac{1}{3} \cdot x_{A}\right) \cdot \eta}{x_{A}+x_{C}-x_{A} \cdot x_{C}}
\end{aligned}
$$

Die Last-Verformungs-Beziehungen sămtlicher Regimes sind im Bild 5.8 graphisch ausgewertet. Das Verhältnis der Biegewiderstände im Einspannbereich und in Feldmitte beträgt für alle Diagramme $M_{R A} / M_{R B}=-2$. Variiert wurden die Zwăngung und die Entfestigungsstabilitătsfaktoren. Bild 5.8a zeigt den zwängungsfreien Fall. 
Mit dem gewăhlten Verhăltnis der Biegewiderstănde erfolgt die Plastifizierung der Bewehrung im Einspannbereich und in Feldmitte gleichzeitig, so dass trotz Entfestigung die Traglast, die mit der Plastizitătstheorie berechnet werden kann, erreicht wird ( $=$ Traglast eines Trăgers mit ideal plastischer Charakteristik). Sind hingegen Zwăngungen vorhanden (Bilder 5.8b und 5.8c), entstehen die plastischen Gelenke nicht gleichzeitig, und infolge von Entfestigung in den Gelenken wird die Traglast nach Plastizitătstheorie nicht erreicht.
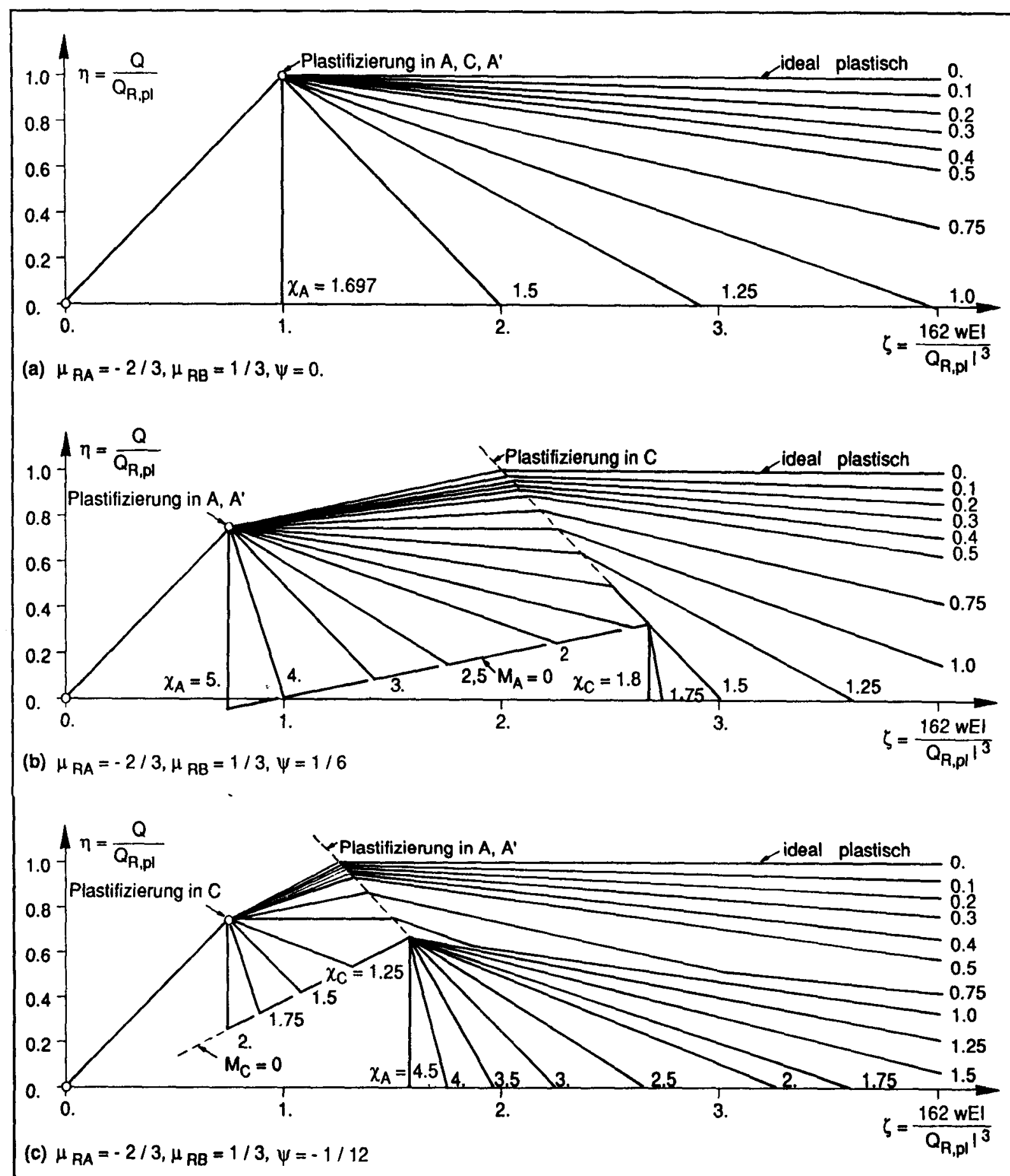

Bild 5.8: Last-Verformungs-Charakteristiken mit verschiedenen Parametern 


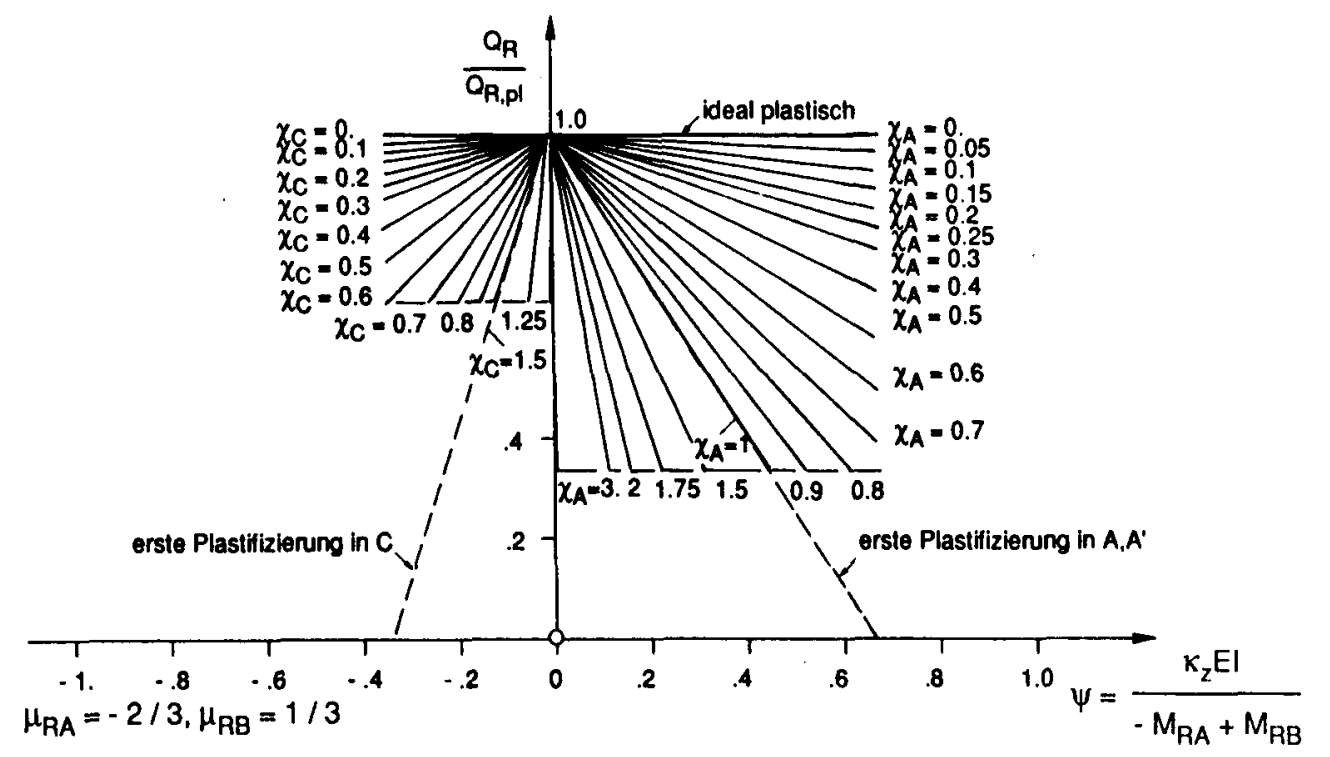

(a) $\mu_{\mathrm{RA}}=-2 / 3, \mu_{\mathrm{RB}}=1 / 3$

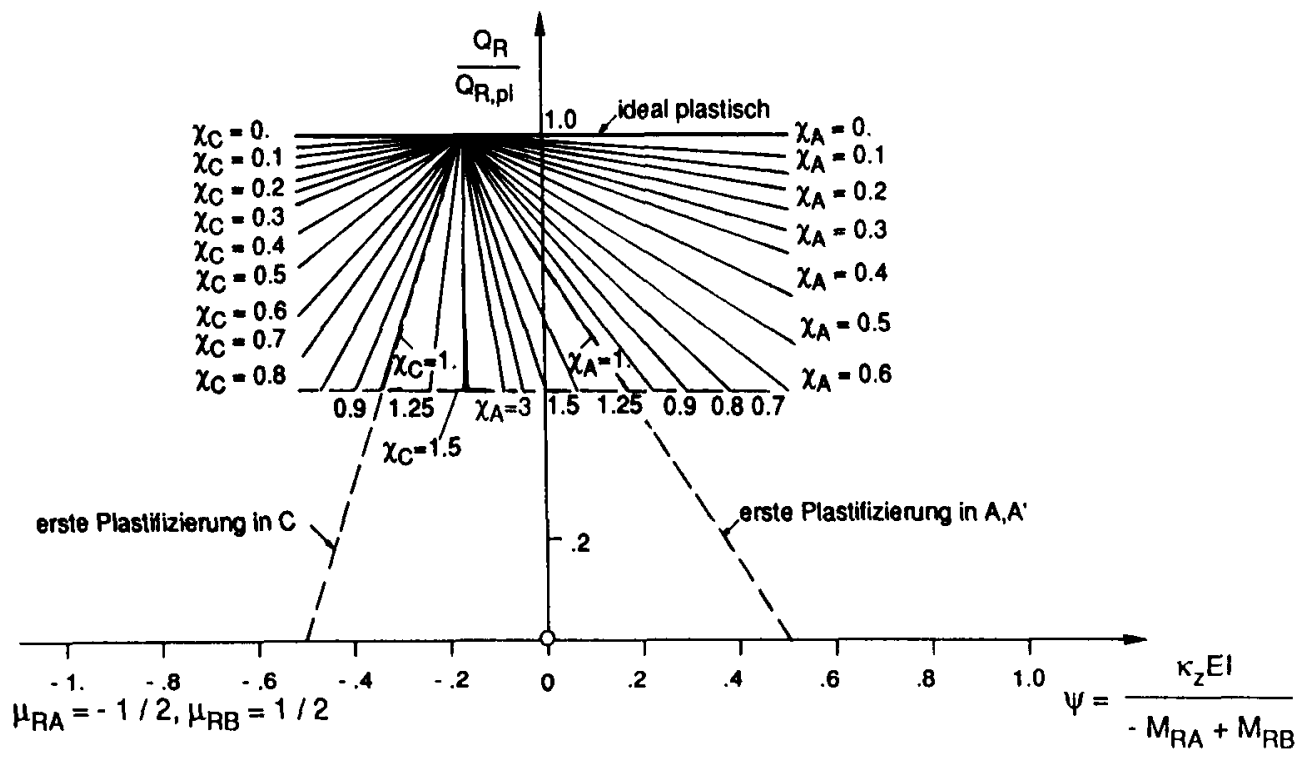

Bild 5.9: Traglast als Funktion des Zwängungsparameters $\psi$

Im Bild 5.9 ist die Traglast als Funktion des Zwăngungsparameters $\psi$ und der Entfestigungsstabilitätsfaktoren graphisch zusammengesteltt. Bild $5.9 \mathrm{~b}$ zeigt den Fall mit dem Verhältnis der Biegewiderstände $\mu_{R A} / \mu_{R B}=M_{R A} M_{R B}=-1 / 1$ (gleiche Bewehrung oben und unten). Bei diesem Widerstandsverhăltnis und im zwăngungsfreien Zustand entstehen die ersten Gelenke zuerst im Einspannbereich, so dass eine Entfestigung in diesen Gelenken eine Abminderung der Traglast verursacht. Es ist zu bemerken, dass die Vereinfachung des Momenten-Krümmungs-Diagrammes den Einfluss der Rissbildung und des Bewehrungsgehaltes nicht berücksichtigt. Im Kapitel 7 wird gezeigt, dass diese Effekte die Fliesslast (Last bei der Bildung des ersten Gelenkes), die Reihenfolge der Gelenkbildung und somit auch die Abminderung der Traglast infolge Entfestigung beeinflussen. 


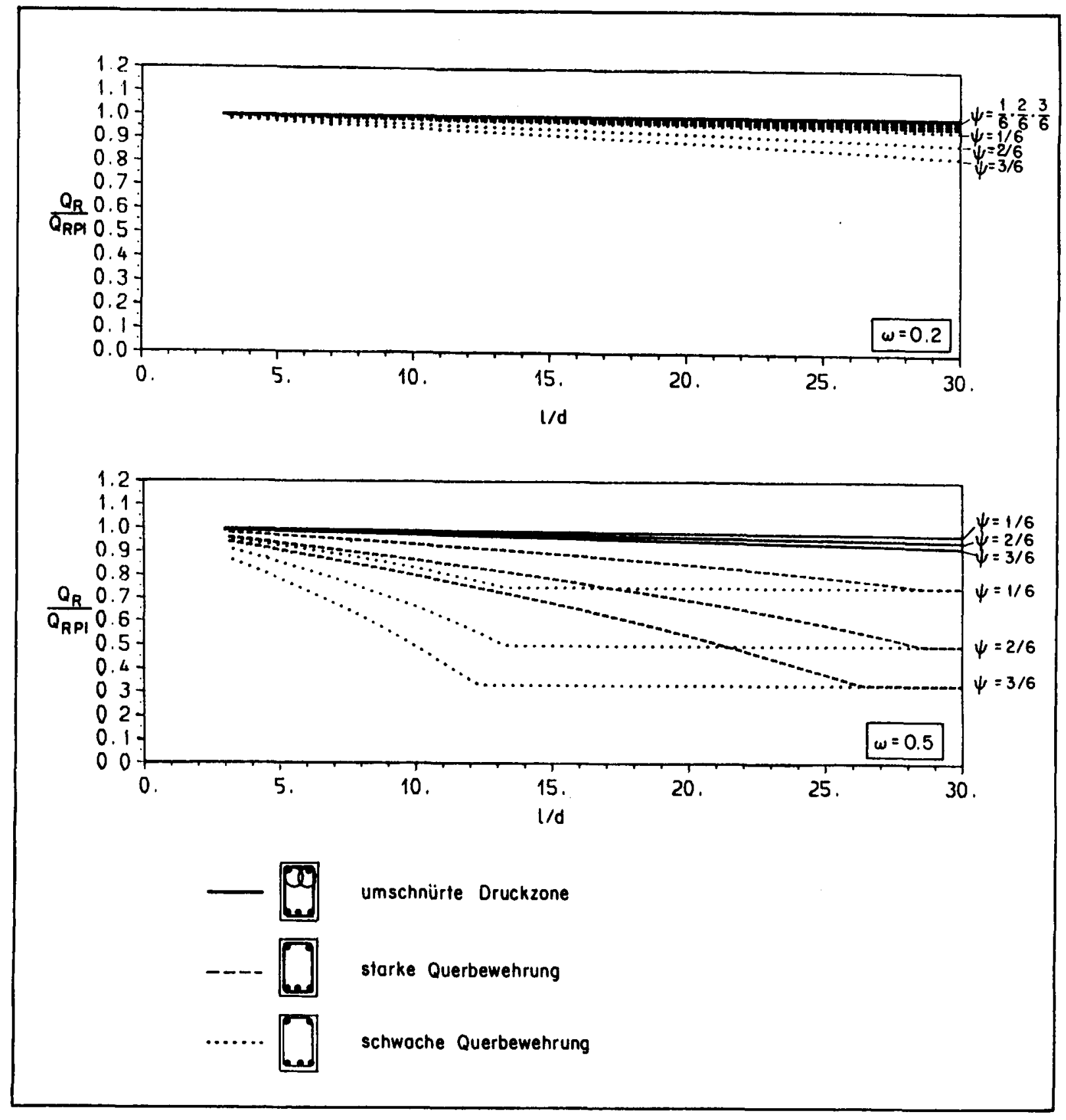

Bild 5.10: Traglast als Funktion der Schlankheit, der Zwăngung, des Lăngsbewehrungsgehaltes und der Querbewehrung $\left(\mu_{R A}=-2 / 3, \mu_{R B}=1 / 3\right)$

Im Bild 5.10 ist die Traglast als Funktion der Schlankheit, der Zwăngung und der Lăngs- und Querbewehrungsgehalte graphisch ausgewertet.

Zur Ermittlung des Entfestigungsstabilitătsfaktors wurden die im Bild 4.14 angegebenen Werte von $\frac{x}{1 / d}$, die aus der Auswertung der Versuche von Base, Read (1965) stammen, eingesetzt. Bild 5.10a zeigt, dass bei Trăgern mit nicht zu grossen Lăngsbewehrungsgehalten $(\omega=0.2)$ die Entfestigung keine zu grosse Abminderung der Traglast verursacht. Nur im Fall einer schwachen Querbewehrung (zu grosser Bügelabstand) und für grosse Zwängungen und Schlankheiten übersteigt diese Abminderung $10 \%$ der Traglast. Bei Trăgern mit starker Lăngsbewehrung ist hingegen der Einfluss der Entfestigung deutlicher. 
Wie Bild 5.10b $(\omega=0.5)$ zeigt, wird im Fall eines Trăgers mit mittlerer Schlankheit $(\ell / \mathrm{d}=15)$ und schwacher Querbewehrung mit $\psi=1 / 6$ die Traglast um $25 \%$ reduziert (mit $\psi=1 / 6$ ist die Beanspruchung infolge Zwăngung gerade $1 / 4$ des Widerstandes im Einspannbereich). Klein ist hingegen die Traglastreduktion, wenn die Druckzone mit einer Umschnürungsbewehrung verstärkt wird.

\subsection{Systeme mit plastischen Verformungen in den Druckgliedern}

Das Last-Verformungs-Verhalten gedrungener Druckglieder ist im Abschnitt 4.2 beschrieben. Oft sind aber die Druckglieder derart schlank, dass deren Verhalten von den Effekten 2. Ordnung stark beeinflusst wird. Dies ist bereits im Abschnitt 2.5 anhand eines einfachen Stab-Feder-Modells beschrieben worden. Als Ergänzung dazu wird das im Bild 5.11 dargestelte System behandelt.
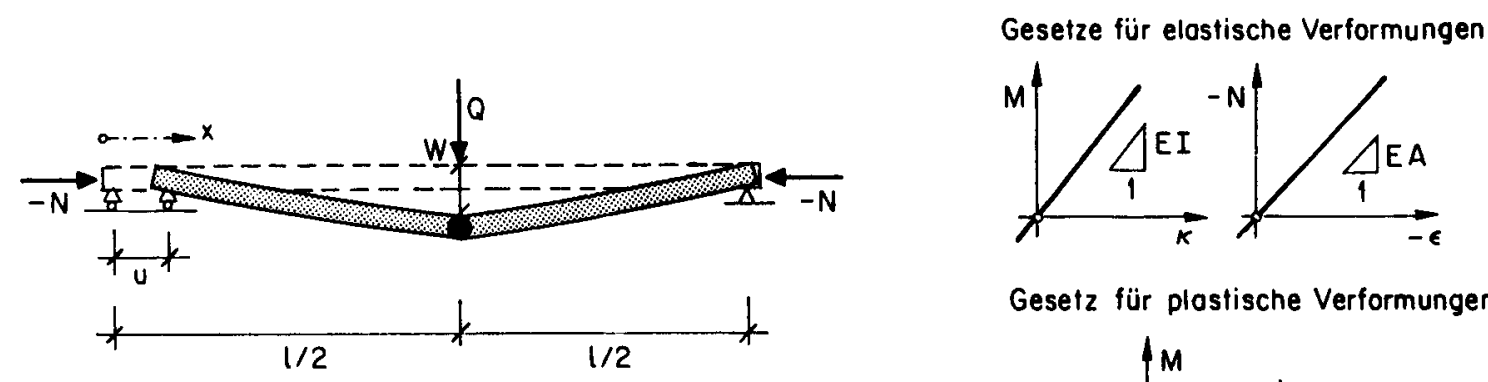

Gesetz für plostische Verformungen

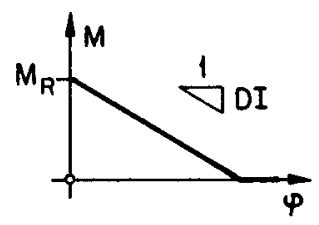

Bild 5.11: System und Materialverhalten

Da nur die wichtigsten Merkmale des Last-Verformungs-Verhaltens aufgezeigt werden sollen, wird das Materialverhalten stark vereinfacht. Insbesondere wird auf die Berücksichtigung der Einflüsse des Biegemomentes auf die Stabverlängerung, der Normalkraft auf die Krümmung und der Normalkraft auf den Momentenwiderstand verzichtet. Folgende dimensionslose Normierungsgrössen werden eingeführt:

- Für die Querlast: $\quad \quad \quad \quad=Q / Q_{R o}$,

wobei $Q_{R o}=$ Traglast des Systems ohne Normalkraft: $Q_{R o}=4 \cdot M_{R} / \ell$

- Für die Normalkraft: $\quad v=N / N_{c r}$,

wobei $N_{c r}=$ elastische Knicklast nach Euler: $\quad N_{c r}=-\pi^{2} \cdot E l / l^{2}$

- Für die Durchbiegung: $\quad \zeta=w \cdot \frac{48 \cdot E I}{Q_{\mathrm{RO}_{0}} \cdot l^{3}}$

- Für die Verkürzung: $\quad \xi=u \cdot \frac{E A}{-N_{c r} \cdot l}$ 
- Für die Schlankheit: $\quad \lambda=\ell \cdot \sqrt{E A T E T}$

- Für den Momentenwiderstand: $\quad \mu=\frac{M_{R} \cdot \ell}{E T}=\frac{M_{R}}{\sqrt{E A \cdot E T}}$

Der Entfestigungsstabilitătsfaktor des Systems ohne Normalkraft betrăgt

$$
x_{0} \quad=\frac{\ell \cdot \mathrm{DI}}{3 \cdot \mathrm{EI}}
$$

Die Differentialgleichung für die Ermittlung der Biegelinie resultiert aus der Gleichgewichtsbedingung:

$$
w^{\prime \prime} \cdot E I-N \cdot w+\frac{Q}{2} \cdot x=0
$$

und lautet in dimensionsloser Form

$$
\zeta^{\prime \prime}\left(\frac{x}{l}\right)+v \cdot \pi^{2} \cdot \zeta\left(\frac{x}{l}\right)+24 \cdot \eta \cdot \frac{x}{l}=0
$$

Die Lơsung dieser Differentialgleichung ergibt sich zu

$$
\zeta\left(\frac{x}{l}\right)=12 \cdot \alpha \cdot \sin \sqrt{v} \cdot \pi \cdot \frac{x}{l}+12 \cdot \beta \cdot \cos \sqrt{v} \cdot \pi \cdot \frac{x}{l}-\frac{24 \cdot \eta}{\pi^{2} \cdot v} \cdot \frac{x}{l}
$$

Die Konstanten $\alpha$ und $\beta$ können aus den Randbedingungen ermittelt werden:

$$
\zeta(0)=0 \quad \rightarrow \quad \beta=0
$$

Im elastischen Zustand gilt mit $\zeta^{\prime}\left(\frac{1}{2}\right)=0$ :

$$
\alpha=\frac{2 \cdot \pi}{(\sqrt{v} \cdot \pi)^{3} \cdot \cos \cdot \sqrt{v} \cdot \frac{\pi}{2}}
$$

und nach der Plastifizierung in Balkenmitte:

$$
\alpha=\frac{1+\frac{12 \cdot \eta \cdot x_{0}}{v \cdot \pi^{2}}}{v \cdot \pi^{2} \cdot \sin \sqrt{v} \cdot \frac{\pi}{2}+6 \cdot \sqrt{v} \cdot \pi \cdot x_{0} \cdot \cos \sqrt{v} \cdot \frac{\pi}{2}}
$$

Zwei Last-Verformungs-Beziehungen sind von Interesse. Die erste beschreibt die Querlast als Funktion der Durchbiegung und lautet mit $\zeta=\zeta\left(\frac{1}{2}\right)$ :

$$
\zeta=12 \cdot \alpha \cdot \sin \sqrt{v} \cdot \frac{\pi}{2} \cdot \frac{12 \cdot \eta}{\pi^{2} \cdot v}
$$


Die zweite Beziehung beschreibt die Normalkraft als Funktion der Verkürzung und wird aus folgender Beziehung hergeleitet:

$$
u \simeq-\frac{N \cdot l}{E A}+\int_{0}^{l} \sqrt{1+w^{\prime 2}} \cdot d x-l \simeq-\frac{N \cdot l}{E A}+\frac{1}{2} \cdot \int_{0}^{l} w^{2} \cdot d x
$$

Sie lautet:

$$
\xi=v+\lambda^{2} \cdot \mu^{2} \cdot\left[\frac{\alpha^{2} \cdot v}{4} \cdot\left(1+\frac{\sin \sqrt{v} \cdot \pi}{\sqrt{v} \cdot \pi}\right)-4 \cdot \eta \cdot \alpha \cdot \frac{\sin \sqrt{v \cdot \frac{\pi}{2}}}{v \cdot \pi^{4}}+\frac{2 \cdot \eta^{2}}{v^{2} \cdot \pi^{6}}\right]
$$

Diese Beziehungen sind in den Bildern 5.12 und 5.13 graphisch ausgewertet. Im Bild 5.12 ist die Querlast $(\eta)$ als Funktion der Durchbiegung $(\zeta)$ im Fall einer konstant wirkenden Normalkraft $(v)$ dargestellt. Mit zunehmendere Normalkraft nehmen die Steifigkeit des Systems im elastischen Bereich und die Querlast $\left(\eta_{R}\right)$ beim Erreichen des Widerstandes in Balkenmitte ab (sog. P- $\Delta$-Effekt, Bild 5.12d). Im plastischen Bereich hat die Normalkraft einen ähnlichen Einfluss wie eine lineare Entfestigung. Bild 5.12e zeigt den Einfluss der Normalkraft $(v)$ und der Duktilităt (Entfestigungsstabilitătsfaktor $\chi_{0}$ des Stabes ohne Normalkraft) auf den Entfestigungsstabilitătsfaktor. Bei der Berechnung dieses Faktors muss in der Energiebilanz auch die von der Normalkraft geleistete Arbeit (Normalkraft mal Verkürzung) berücksichtigt werden.

Der Fall mit konstanter Querlast ist im Bild 5.13 behandelt; die Normalkraft $(v)$ ist als Funktion der Verkürzung $(\xi)$ mit der Querlast $(\eta)$ als Parameter dargestellt. Da der Einfluss der Schlankheit schon im Abschnitt 2.5 diskutiert wurde, wird hier nur mit der Schlankheit $\lambda=100$ gerechnet. In den vier dargestellten Diagrammen werden der Entfestigungsstabilitătsfaktor $\chi_{0}$ und der normierte Momentenwiderstand $(\mu)$ variiert. Für kleine Momentenwiderstănde (Bilder 5.13a und b) erfolgt eine Entfestigungsinstabilităt; die gespeicherte Energie (Stauchung des Stabes) und die Arbeit der Querlast sind grősser als die Energie, die im Biegegelenk dissipiert werden kann. Bei konstanter Verkürzung $(\xi)$ nimmt die Auslenkung $(\zeta)$ zu und die Normalkraft nimmt ab. Erst bei grossen Auslenkungen kann wieder ein Gleichgewichtszustand gefunden werden; die Normalkraft hat aber (bei kleinen $\mu$ und grossen $\chi_{0}$ ) schon stark abgenommen. 


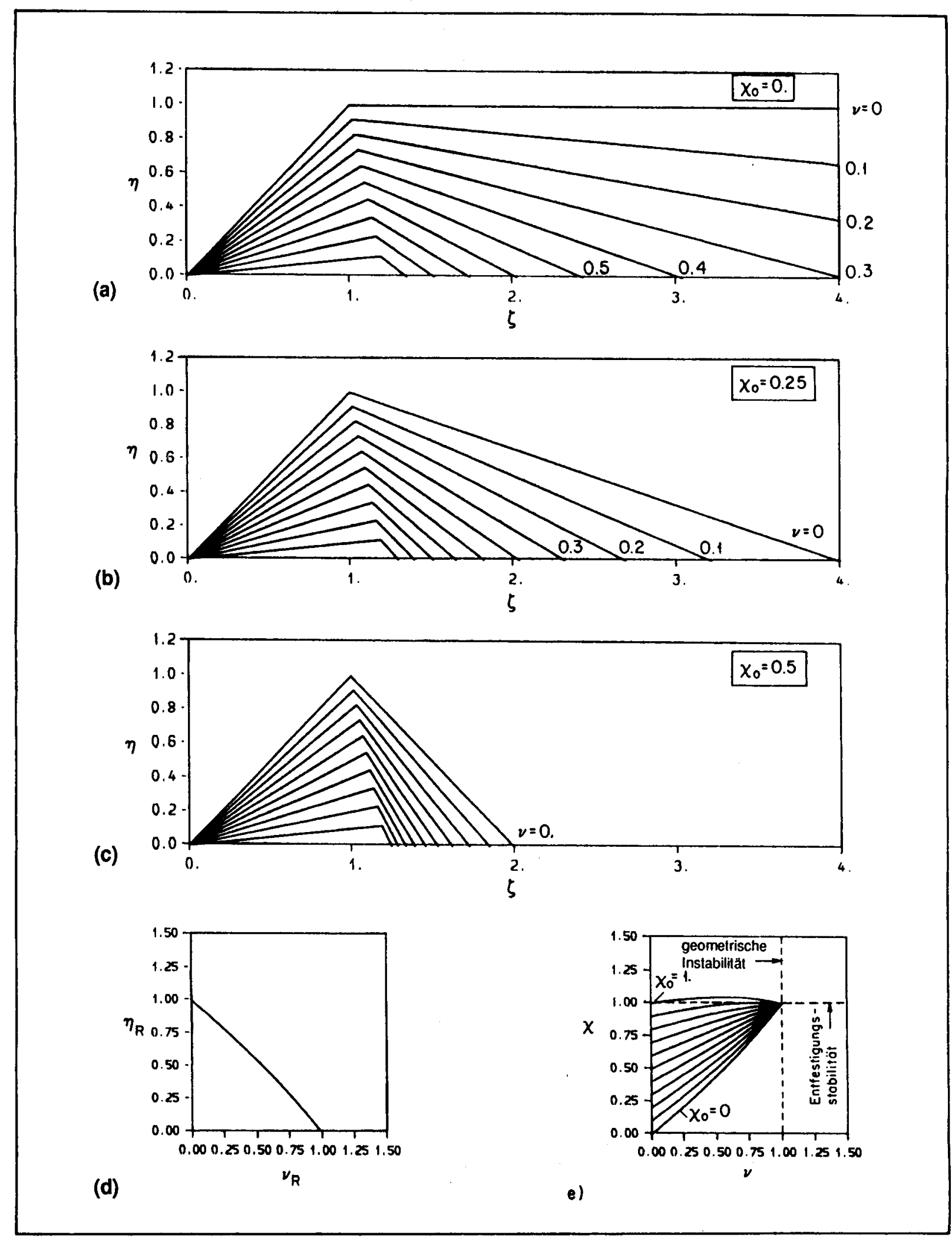

Bild 5.12: (a) bis (c) Querlast ( $\eta$ ) als Funktion der Durchbiegung ( $\zeta$ ) im Fall einer konstanten Normalkraft $(v)$ mit verschiedenen Entfestigungsstabiitătsfaktoren $x_{0}$; (d) Traglast $\left(\eta_{R}\right)$ als Funktion der Normalkraft $\left(v_{R}\right)$; (e) Entfestigungsstabilitătsfaktor $\chi$ als Funktion der Normalkraft. 


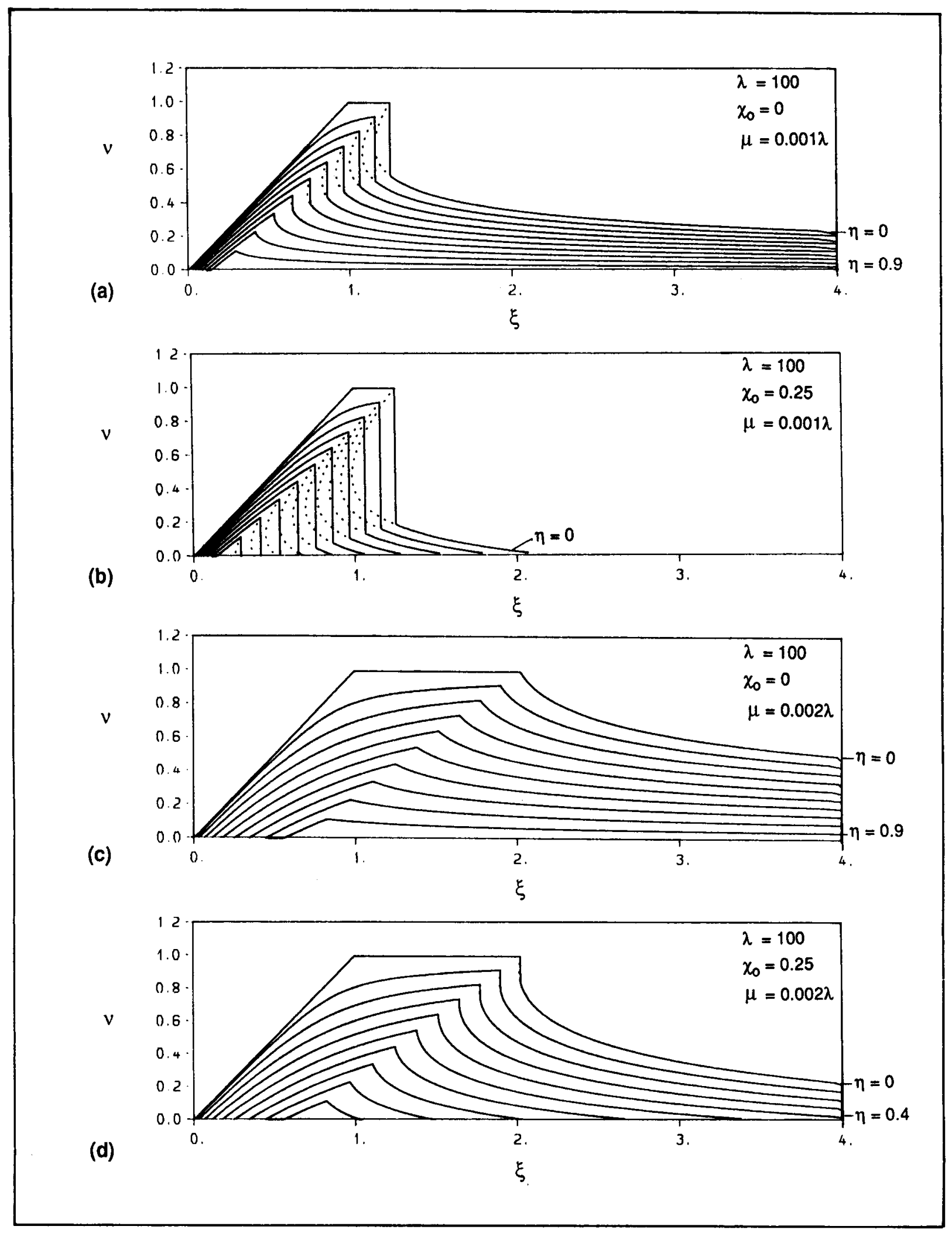

Bild 5.13: Normalkraft $(v)$ als Funktion der Verkürzung $(\zeta)$ im Fall einer konstanten Querlast ( $\eta$ ) 


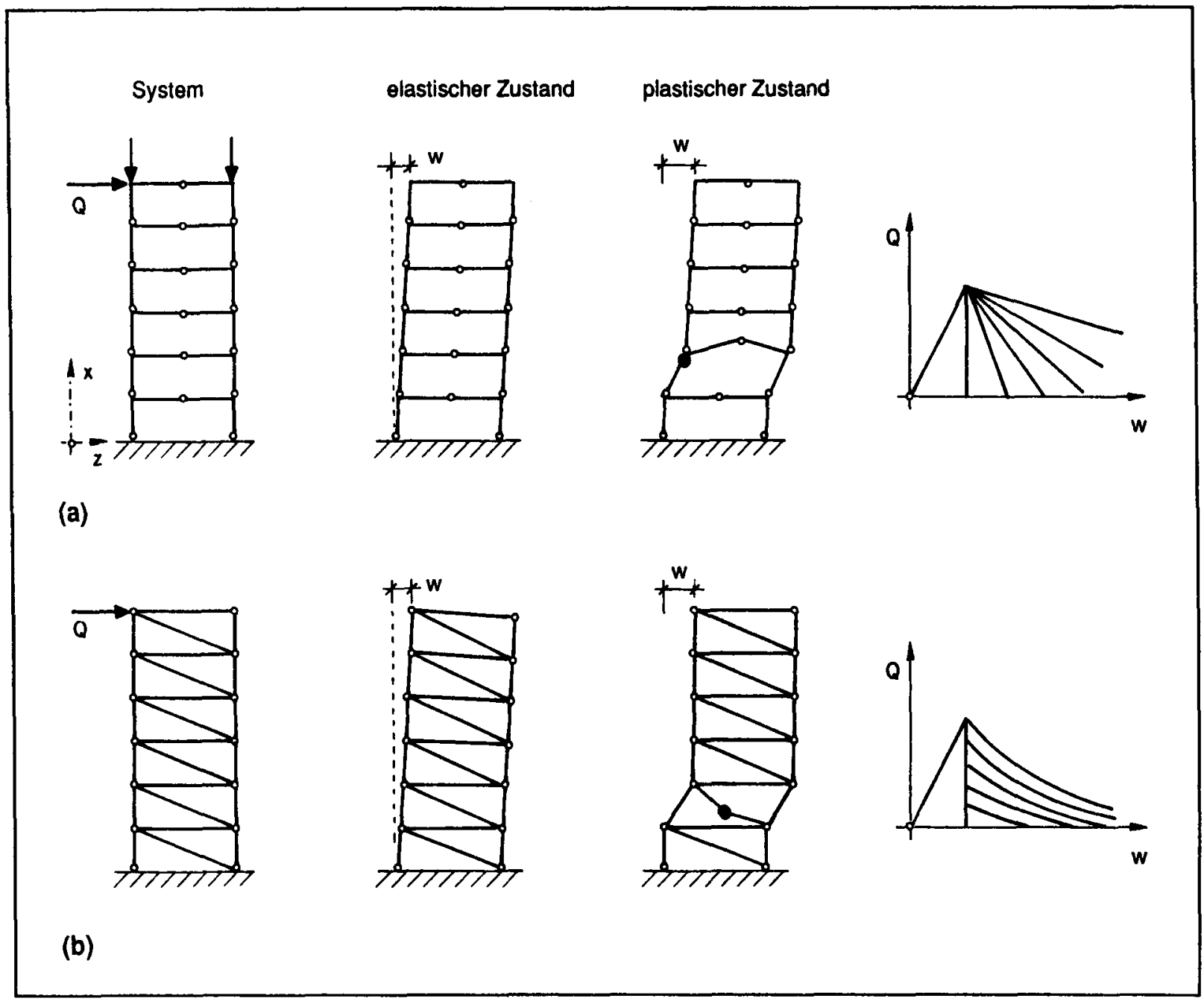

Bild 5.14: Statisch unbestimmte Systeme mit Verformungslokalisierung nach plastischem Knicken eines Druckgliedes

Bevor Fälle untersucht werden, bei welchen Druckglieder als Elemente statisch unbestimmter Systeme wirksam sind, muss noch erwăhnt werden, dass in statisch bestimmten Systemen mit mehreren Elementen in Serie das Versagen eines Druckgliedes zu einer Verformungslokalisierung mit rascher Entfestigung des Systems führt. Bild 5.14 zeigt dies am Beispiel zweier mehrstöckiger Rahmen. Wăhrend in einem Stockwerk eine Entfestigung stattfindet, wird bei der Entlastung der weiteren Stockwerke die elastisch gespeicherte Energie freigegeben. Im Fall a) von Bild 5.14 entspricht die Last-Verformungs-Charakteristik des Systems derjenigen eines Druckstabes, bei dem die Querlast als Funktion der Auslenkung beschrieben wird. Es resultien somit auch in diesem Fall ein bilineares Verhalten mit dem Entfestigungsstabilitătsfaktor als Funktion der Systemsteifigkeit, des Entfestigungsmoduls im plastischen Gelenk sowie der Normalkraft (P- $\Delta$-Effekt, siehe Bild 5.12). Im Fall b) hingegen erfolgt die plastische Verformung in einem nur mit Normalkraft beanspruchten Druckstab, so dass die Last-Verformungs-Charakteristik des Systems derjenigen des im Bild 5.13 dargestelten Falles entspricht (einfaches plastisches Knicken eins Druckgliedes). Sie ist gekennzeichnet durch eine Instabilităt (für übliche Schlankheiten und Momentenwiderstănde), nach welcher eine Restfestigkeit aktiviert werden kann. Der Entfestigungsstabilitătsfaktor, und somit auch die Gefahr eines Sprödbruchs, kann vermindert werden, wenn ein zweites parallel gekoppeltes System angeordnet wird. Wie aus Bild 5.15 ersichtlich, nimmt dieses System denjenigen Beanspruchungsanteil auf, der nach der Bildung des ersten plastischen Gelenkes vom Primärsystem nicht mehr aufgenommen werden 
kann (Entfestigung). Diese Beanspruchung wird in die benachbarten Stockwerke eingeleitet, so dass auch dort plastische Gelenke entstehen.

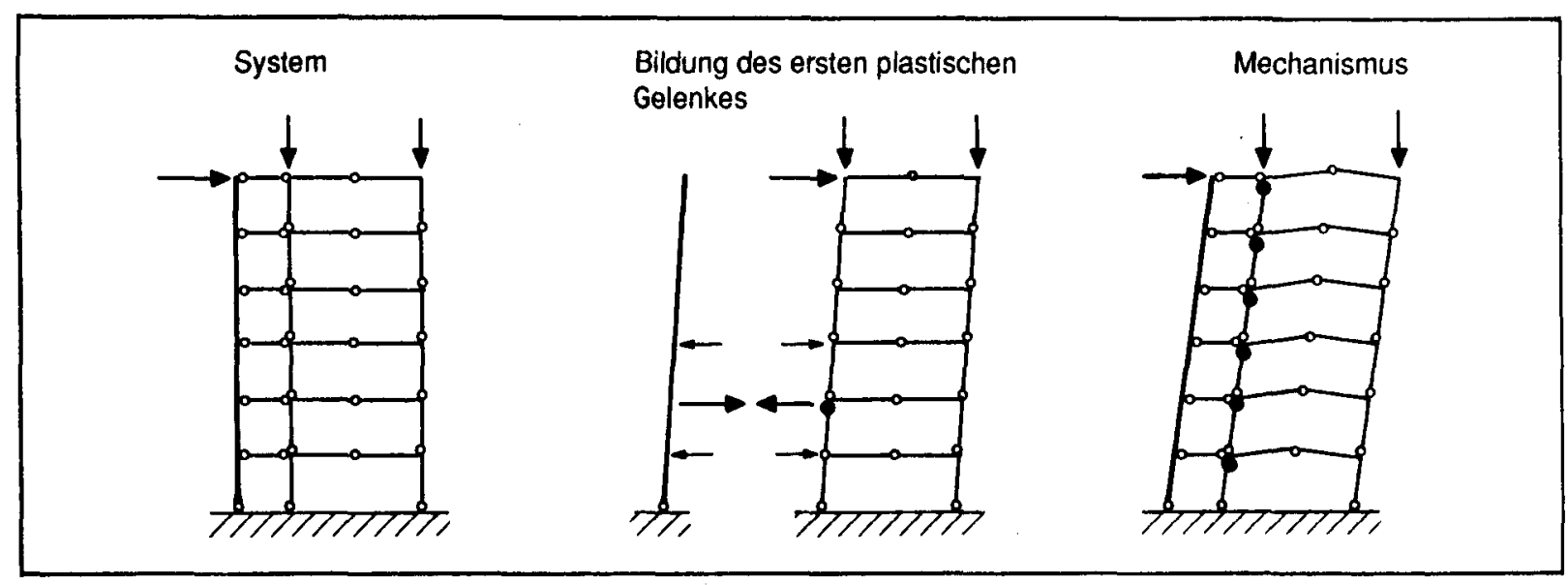

Bild 5.15: Verhinderung der Verformungslokalisierung durch parallel gekoppeltes System

Die plastifizierte Zone pflanzt sich fort, bis ein Mechanismus entstanden ist. Da mit mehr plastischen Gelenken auch mehr Energie dissipiert werden kann, nimmt der Entfestigungsstabilitătsfaktor ab. Konstruktiv wird dies erreicht, wenn ein Kern mit genügendem Widerstand angeordnet wird, oder wenn in den Stützen keine konstruktiven Gelenke vorhanden sind und der Stützenwiderstand so gross ist, dass dort keine plastischen Gelenke entstehen. Ein solches System zeigt eine grosse Analogie mit dem im Abschnitt 4.4 behandelten schubbeanspruchten Balken, bei welchem die Verformungslokalisierung infolge Stegentfestigung von den Gurten verhindert wird.

Als erstes Beispiel wird der von Cranston (1965) experimentell untersuchte Stahlbetonrahmen (Versuch P7) behandelt. Das System und die Abmessungen sind im Bild 5.16a dargestellt. Die Bilder $5.16 \mathrm{c}$ und $5.16 \mathrm{~d}$ zeigen die experimentell ermittelten Momenten-Rotations-Diagramme für die zwei Zonen, in denen plastische Verformungen entstanden sind ( $C$ und $\left.B^{\prime}\right)$. Daraus lassen sich die Biegesteifigkeit El, die Momentenwiderstände und die Entfestigungsmodule DI ermitteln. Es wurde mit einer reduzierten Messlănge $l '$ gerechnet, da das Biegemoment im Messbereich nicht konstant ist. Wie aus den Bildern ersichtlich ist, weicht das angenommene bilineare Verhalten (gestrichelte Linie) vom effektiven Verhalten sehr wenig $a b$. Die beiden Vertikallasten $Q$ und die Horizontallast $H$ wirken immer im gleichen Verhăltnis; beim Versuch $P 7$ betrug es $H / Q=0.182$.

Das System ist einfach statisch unbestimmt und kann mit den üblichen Methoden der Baustatik untersucht werden. Für die Rahmenstützen lauten die Gleichgewichtsbedingungen:

$$
\begin{array}{ll}
\text { Stütze A-B: } & M(z)=\left(Q-\frac{H \cdot h}{l}\right) \cdot u+\left(\frac{H}{2}-R\right) \cdot x=-u^{\prime \prime} \cdot E l \\
\text { Stütze A'-B': } & M\left(z_{1}\right)=\left(Q+\frac{H \cdot h}{l}\right) \cdot u_{1}+\left(\frac{H}{2}+R\right) \cdot x=-u_{1}^{\prime \prime} \cdot E I
\end{array}
$$


und die Lösungen:

$$
\begin{aligned}
& u(z)=a \cdot \sin \omega x-\frac{H \cdot x}{2 \cdot\left(Q-\frac{H \cdot h}{l}\right)}+\frac{R \cdot x}{Q-\frac{H \cdot h}{l}} ; \quad \text { mit } \omega=\sqrt{\frac{Q-H \cdot h / l}{E T}} \\
& u_{1}(z)=a_{1} \cdot \sin \omega^{\prime} h-\frac{H \cdot x}{2 \cdot\left(Q+\frac{H \cdot h}{l}\right)}-\frac{R \cdot x}{Q+\frac{H \cdot h}{l}} ; \text { mit } \omega^{\prime}=\sqrt{\frac{Q+H \cdot h / l}{E T}}
\end{aligned}
$$

Im Riegel ist die Normalkraft so klein, dass die Effekte 2. Ordnung vemachlässigt werden können. Die Integrationskonstanten a und $a_{1}$ sowie die überzăhlige Grösse $R$ können aus den folgenden Bedingungen erhalten werden:

$u(h)=u_{1}(h) \quad$ (keine Zwăngung und keine Lăngenănderung des Riegels)

$u^{\prime}(h)=w^{\prime}(0)+\varphi_{B} ; \quad$ mit $\varphi_{B}=0, \quad$ oder $\varphi_{B}=\frac{M_{R B}-M_{B}}{D T_{B}}$

$u_{1}^{\prime}(h)=w^{\prime}(l)+\varphi_{B}^{\prime} ; \quad$ mit $\varphi_{B}^{\prime}=0, \quad$ oder $\varphi_{B}^{\prime}=\frac{M_{R B}-M_{B}^{\prime}}{D T_{B}}$
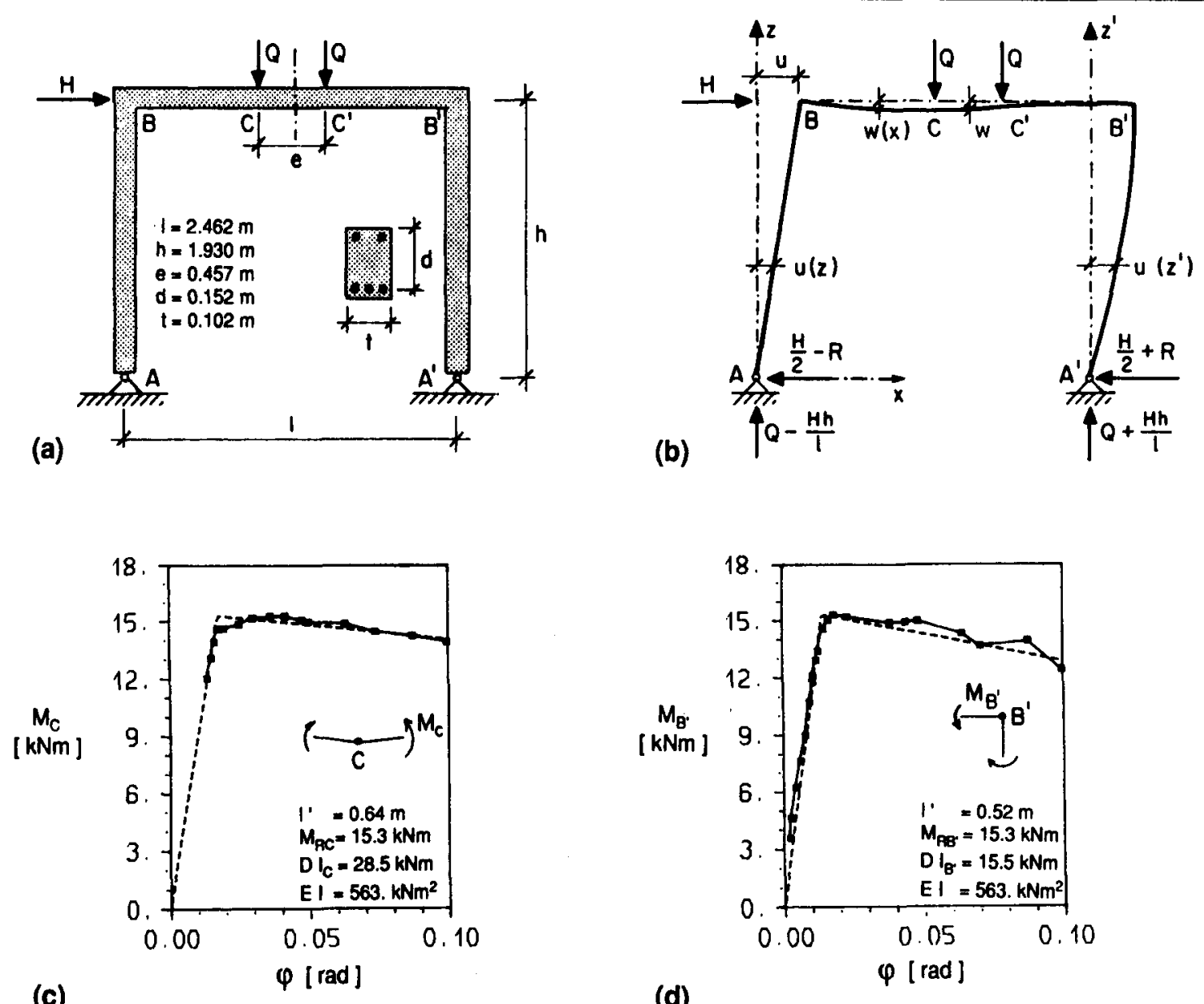

Bild 5.16: Von Cranston (1965) untersuchter Stahlbetonrahmen: (a) System und Abmessungen; (b) statisches System; (c) und (d) Materialverhalten. 


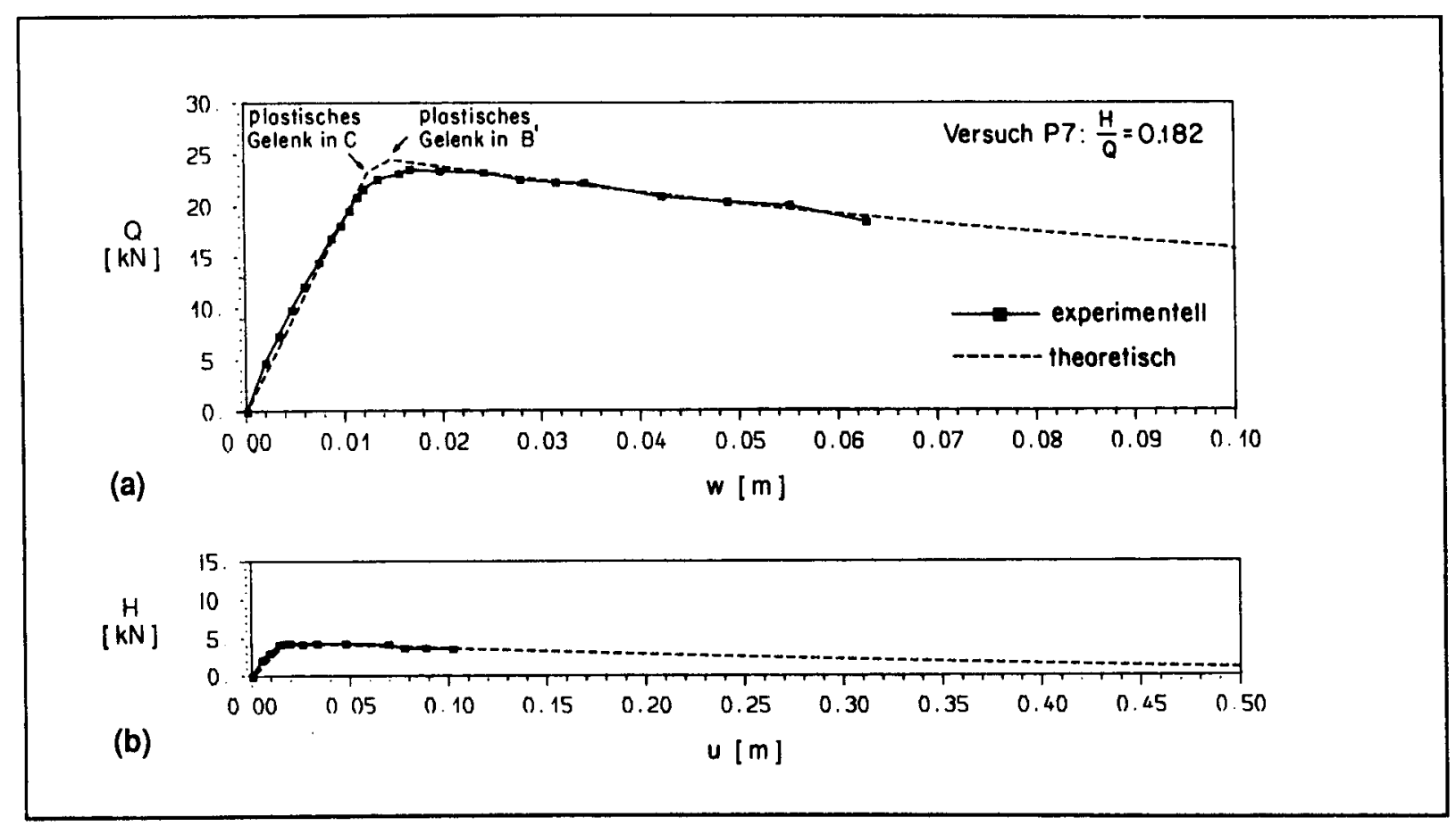

Bild 5.17: Experimentell ermittelter und theoretischer Verlauf der Last-Verformungs-Beziehungen

Auf eine algebraische Herleitung der Resultate wird hier verzichtet. Diese sind im Bild 5.17 für den experimentell untersuchten Fall mit $H / Q=0.182$ in Form von Last-Verformungs-Kurven graphisch ausgewertet. Aus dem Bild geht hervor, dass die Uebereinstimmung zwischen den experimentellen und den theoretischen Resultaten sehr gut ist. Die theoretisch ermittelten Last-Verformungs-Beziehungen zeigen, dass die zwei plastischen Gelenke, die zur Bildung eines Mechanismus erforderlich sind, nicht gleichzeitig entstehen. Zuerst bildet sich ein Gelenk in $C$, und nach einem kleinen Lastzuwachs entsteht auch in B' ein plastisches Gelenk. Die Reihenfolge der Gelenkbildung und die Umlagerung der inneren Kräfte bis zur Bildung des Mechanismus sind vom Verhältnis Horizontallast/Nertikallast und von den Zwängungen (die in dieser Untersuchung nicht berücksichtigt wurden) abhängig. Dies ist im Bild 5.18a, in dem die H-Q-Interaktionsdiagramme für die Entstehung des ersten und des zweiten Gelenkes dargestellt sind, erkennbar. Im gleichen Bild ist auch die mit der Plastizitätstheorie ermittelte Traglast eingetragen.

Die Traglast kann auf einfache Art mit der Mechanismusmethode ermittelt werden:

- Mechanismus mit Gelenken in B' und B:

innere Arbeit:

$$
-A_{i}=M_{R B} \cdot \varphi_{B}+M_{R B^{\prime}} \cdot \varphi_{B^{\prime}} ; M_{R B}=M_{R B^{\prime}}
$$

kinematische Vertrăglichkeit:

$$
\varphi_{\mathrm{B}}=\varphi_{\mathrm{B}^{\prime}}
$$

äussere Arbeit:

$$
A_{a}=H_{R} \cdot h \cdot \varphi_{B}
$$


Prinzip der virtuellen Arbeit: $\quad A_{a}+A_{i}=0 \quad \rightarrow \quad H_{R}=\frac{M_{R B}+M_{R B^{\prime}}}{h}$

- Mechanismus mit Gelenken in B' und C:

innere Arbeit:

$$
-A_{i}=M_{R B^{\prime}} \cdot \varphi_{B^{\prime}}+M_{R C} \cdot \varphi_{C}
$$

kinematische Vertrăglichkeit: $\quad \varphi_{B^{\prime}}=\varphi_{C}$

äussere Arbeit:

$$
A_{a}=Q_{R} \cdot \frac{l-e}{2 \cdot l} \cdot l \cdot \varphi_{C}+H_{R} \cdot h \cdot \frac{l+e}{2 \cdot l} \cdot \varphi_{C}
$$

Prinzip der virtuellen Arbeit:

$$
A_{a}+A_{i}=0 \rightarrow Q_{R} \cdot \frac{l-e}{2 \cdot l}+H_{R} \cdot \frac{l+e}{2 \cdot l} \cdot h=M_{R B^{\prime}}+M_{R C}
$$
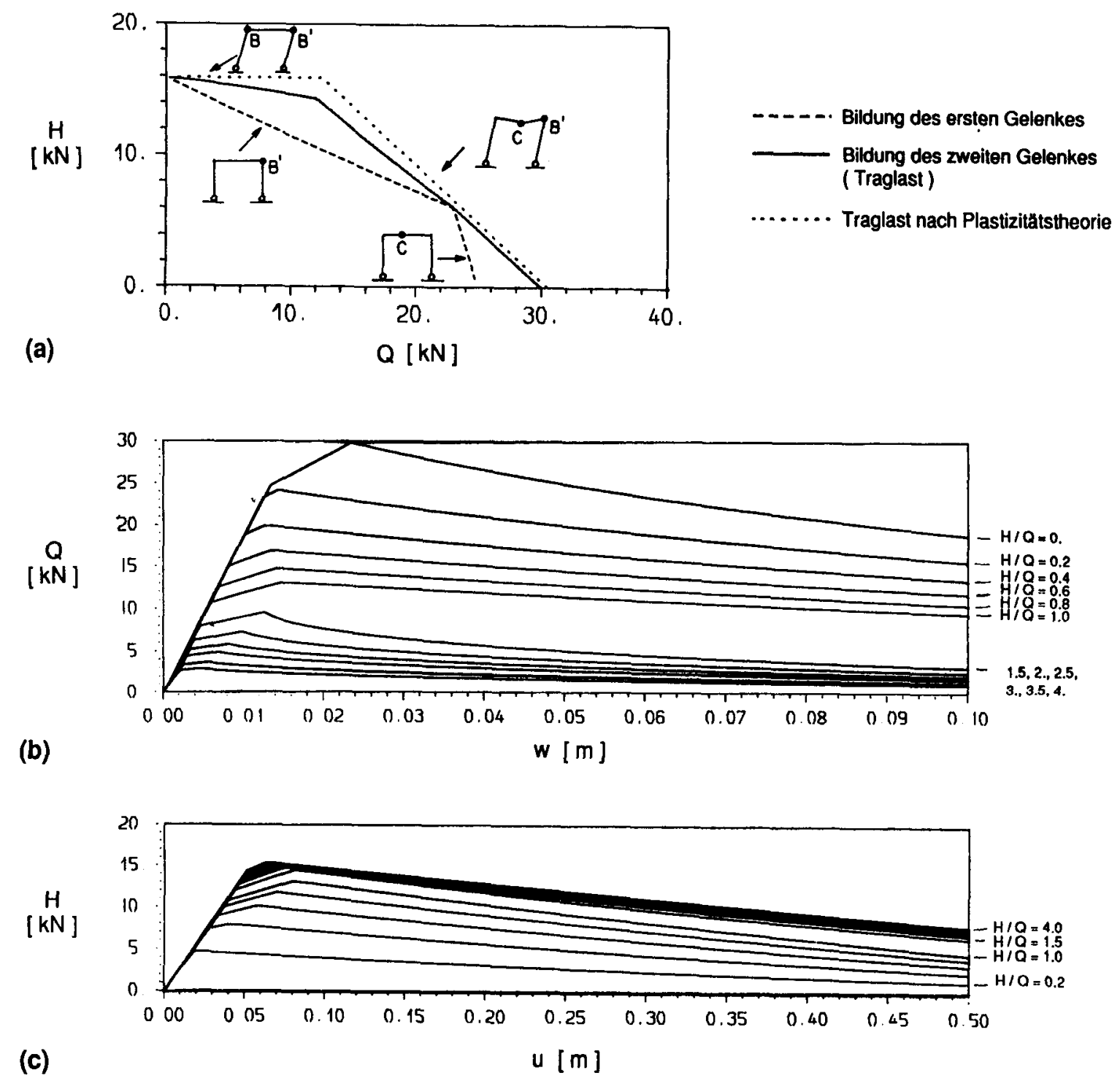

Bild 5.18: Interaktions-Diagramm Horizontallast-Vertikallast und Last-Verformungs-Kurven für verschiedene Lastverhăltnisse 
Wie Bild 5.18a zeigt, bewirkt die Vernachlässigung der Materialentfestigung und der Effekte 2. Ordnung (Plastizitătstheorie) in diesem Fall keine zu grosse Ueberschătzung der Traglast. Die Bilder 5.19 und 5.20 zeigen die Interaktions-Diagramme und die Last-Verformungs-Beziehungen im Fall verdreifachter Entfestigungsmodule bzw. verdoppelter Schlankheit (bei gleicher statischer Höhe wurden alle anderen Abmessungen verdoppelt). Die beiden Bilder zeigen, dass die Materialentfestigung und die Effekte 2. Ordnung ăhnliche Einflüsse auf die Traglast haben, und dass in diesen Fällen die Anwendung der Plastizitătstheorie zu einer zu grossen Ueberschătzung der Traglast führen kann. Es ist zu bemerken, dass der Entfestigungsmodul stark von der Verbügelung des Trägers und von der konstruktiven Ausbildung des Rahmenknotens abhăngig ist. Der Einfluss der Materialentfestigung kann somit klein gehalten werden, indem eine genügend starke Querbewehrung angeordnet wird und die Details sauber konstruiert werden. Der Einfluss 2. Ordnung ( $P$ - $\Delta$-Effekt) ist hingegen stark von der Schlankheit abhängig.

Die Last-Verformungs-Kurven (Bilder 5.18, 5.19, 5.20 b) und c)) zeigen, dass die Entfestigung stark vom Bruchmechanismus abhăngig ist. Dies ist besonders im Bild 5.19b gut ersichtlich. Mit $\mathrm{H} / \mathrm{Q}$ < 1.17 entsteht ein Mechanismus mit plastischen Gelenken in B' und C. In diesem Fall resultiert die Durchbiegung vor allem aus der Biegung des Riegels, so dass dieses Problem mit dem untersuchten Druckstab mit konstanter Normalkraft und zunehmender Querlast verwandt ist (Bild 5.12). Mit $H / Q>1.17$ entsteht ein Mechanismus mit plastischen Gelenken in B und B', so dass die Durchbiegung vor allem auf die Schrägstellung der Stützen zurückzuführen ist. Gleich wie beim Druckstab, bei dem die Normalkraft erhöht wird (Bild 5.13), resultiert auch in diesem Fall eine rasche Entfestigung des Systems. 


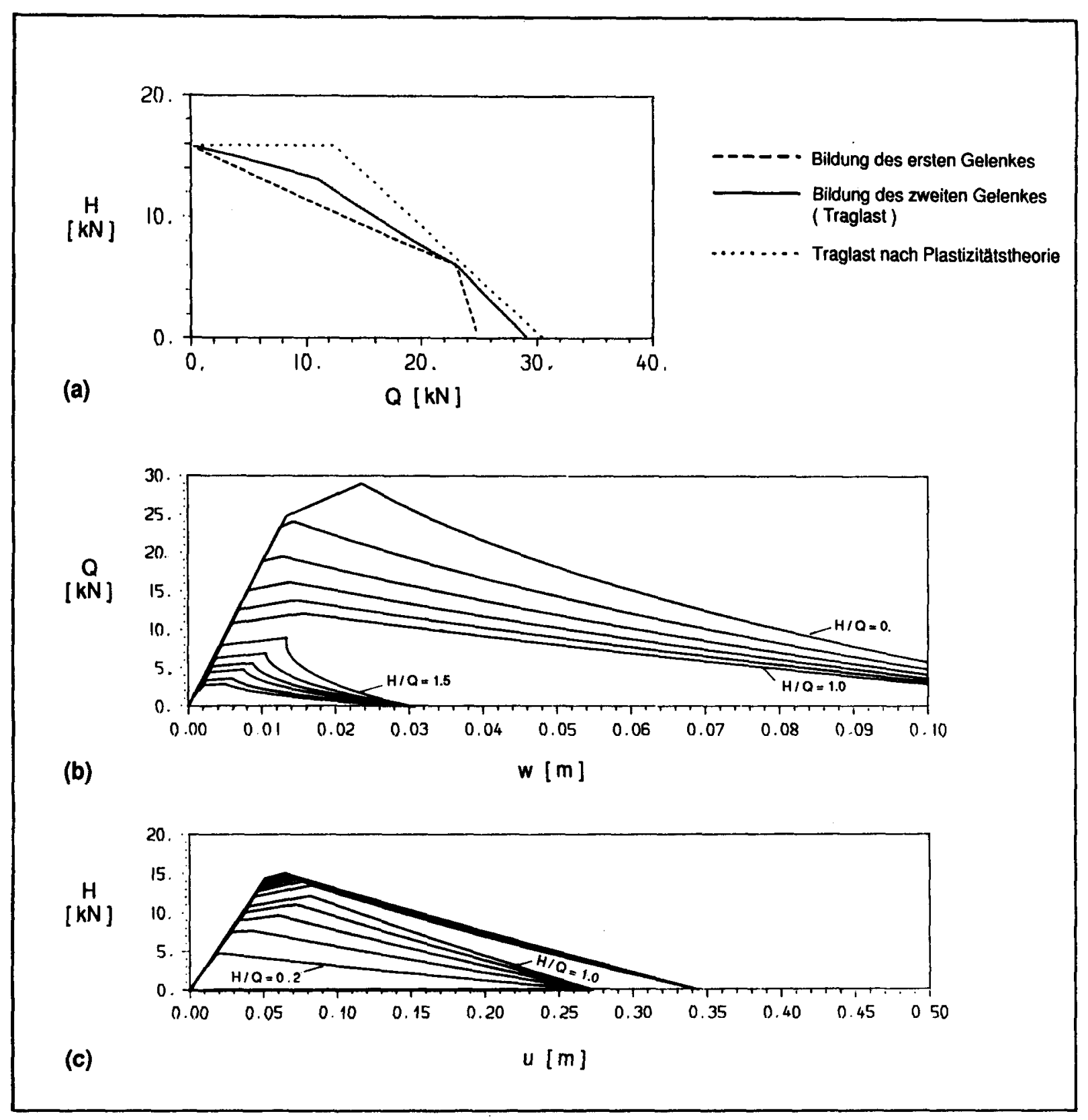

Bild 5.19: Interaktions-Diagramm und Last-Verformungs-Beziehungen im Fall verdreifachter Entfestigungsmodule 


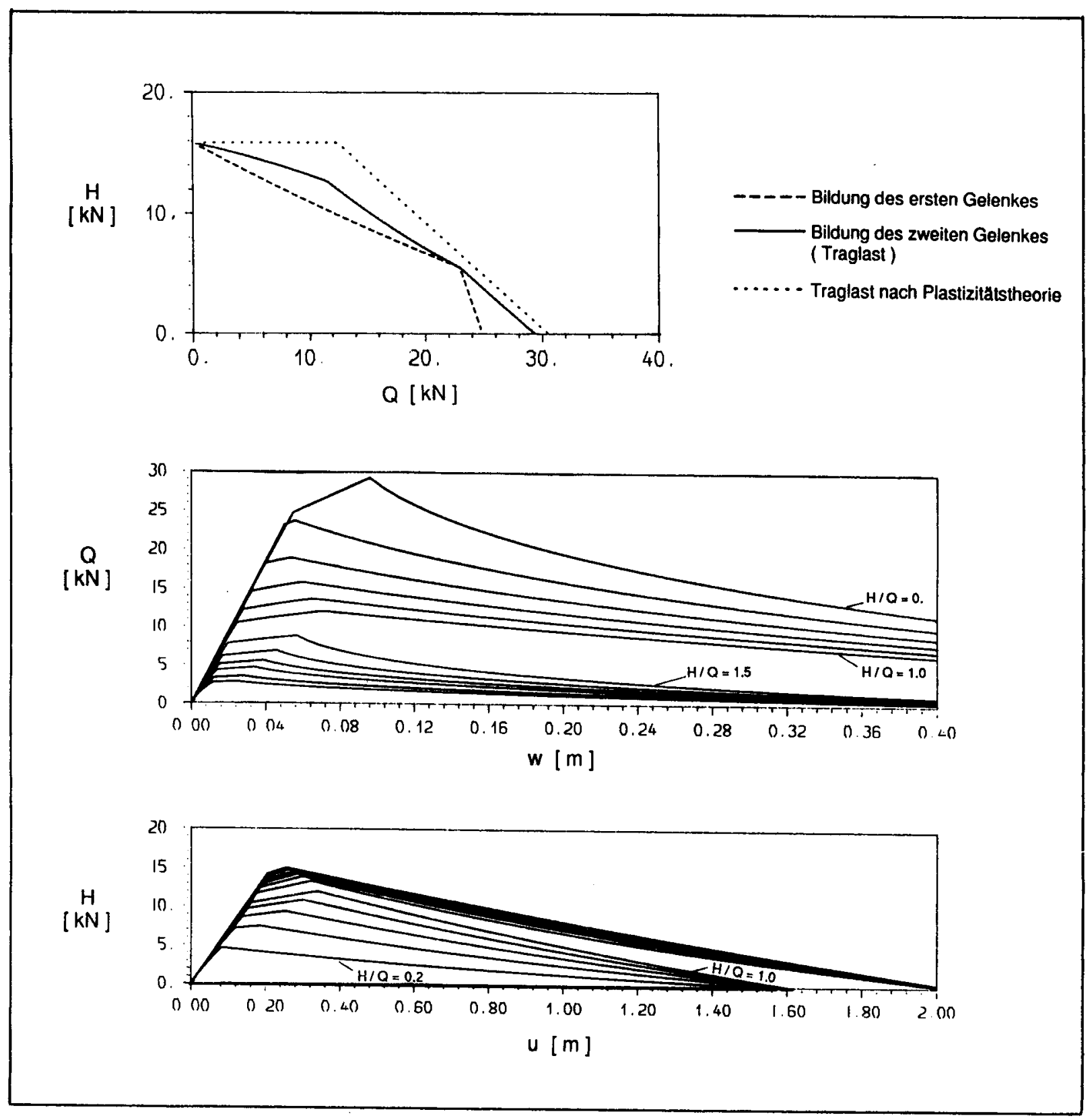

Bild 5.20: Interaktions-Diagramm und Last-Verformungs-Beziehungen im Fall einer verdoppelten Schlankheit

Als zweites Beispiel wird ein weiteres System untersucht, bei dem die charakteristische Verformung von der Verkürzung eines Druckgliedes stark beeinflusst wird. Bild 3.21 zeigt das System und die zwei möglichen Brucharten, bei denen Energie in der Stütze dissipiert wird. 


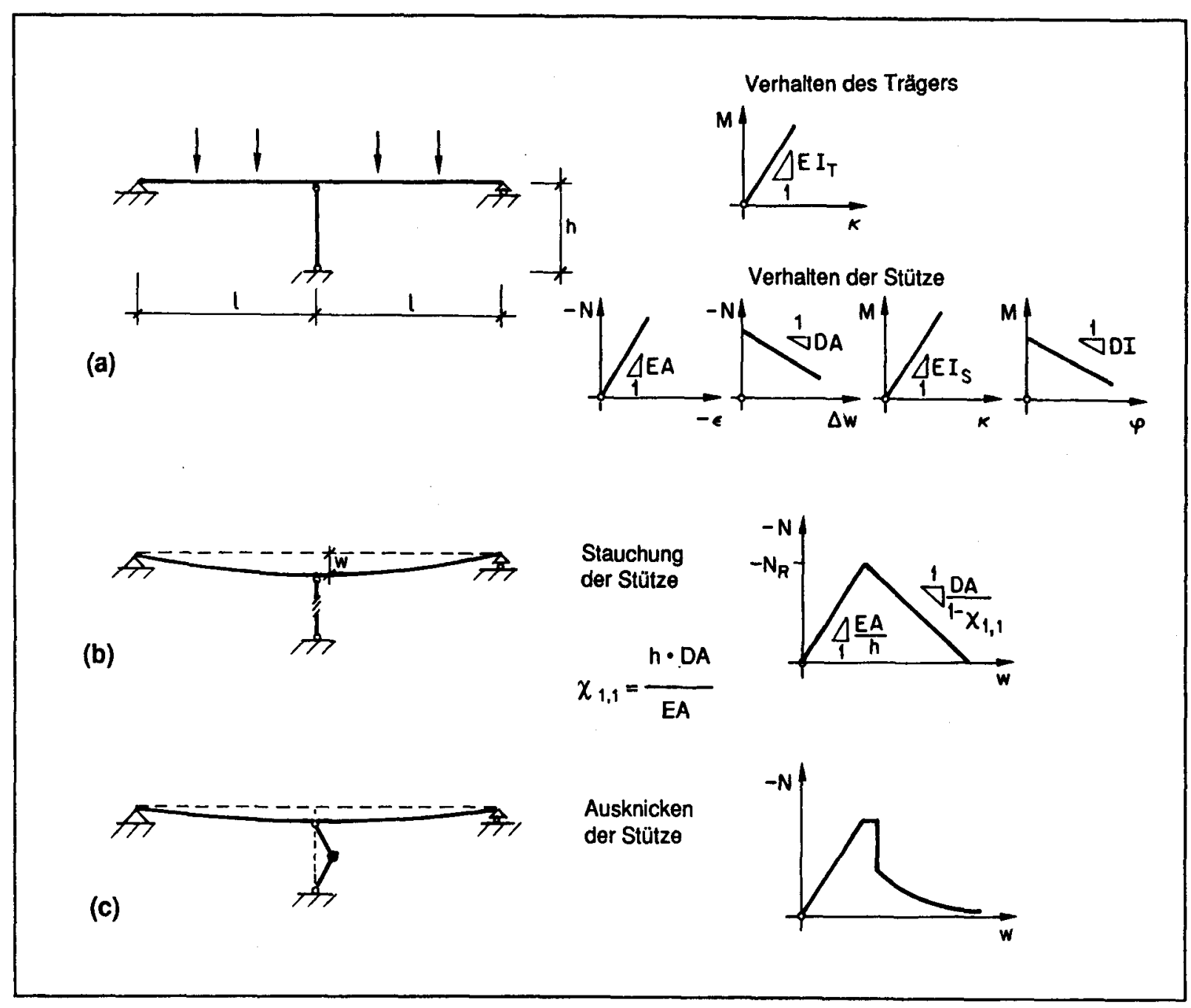

Bild 5.21: Statisch unbestimmtes System mit Dissipation im Druckglied: (a) System und Materialverhalten; (b) Stauchung der Stütze; (c) Ausknicken der Stütze.

Wird die Druckfestiggkeit der Stütze erreicht, dann erfolgt bei gewöhnlicher Lăngsbewehrung und üblichen Querbewehrungsgehalten eine Verformungslokalisierung. Wie im Abschnitt 3.1 dargelegt, verursacht eine derartige plastische Verformung eine lokale Reduktion der Biegesteifigkeit, was zum Ausknicken der Stütze führen kann. Wird angenommen, dass die Stütze nicht ausknicken kann, da sie gehalten ist, kőnnen die Entfestigungsstabilitătsfaktoren aus Abschnitt 3.2 (Bild 3.11d) für eine unbewehrte Săule und aus Abschnitt 4.2 (Bilder 4.4e und 4.5e) für querbewehrte Stützen entnommen werden. Aus diesen Diagrammen können folgende Grenzschlankheiten $h / d$ ( $h=H o ̈ h e, d=D i c k e$ der Stütze) für eine Entfestigungsinstabilităt ermittelt werden:

- unbewehrte Stütze

$$
\begin{array}{ll}
f_{c}=30.0 \mathrm{MPa}: & h / d \simeq 7 \\
f_{c}=60.0 \mathrm{MPa}: & h / d \simeq 4
\end{array}
$$


- runde Stütze mit Querbewehrung

$$
a_{\mathrm{q}}>0.1: \quad h / d \simeq 30
$$

- quadratische Stütze mit einfacher Querbewehrung

$$
a_{\mathrm{t}}>0.1: \quad h / d=20
$$

Zur Beantwortung der Frage, ob eine Stütze in einem statisch unbestimmten System mit Hilfe der Plastizitătstheorie bemessen werden kann, wird hier untersucht, ob nach dem Erreichen der Stützenfestigkeit die Last noch weiter erhöht werden kann oder, ob mit dem Bruch der Stütze die Traglast des Systems erreicht ist. Aufgrund der Ausführungen im Abschnitt 5.1 kann diese Untersuchung erfolgen, indem im System eine Bindung gelöst, eine überzăhlige Grösse eingeführt und deren Entfestigungsstabilitătsfaktor bestimmt wird (Bild 5.22).

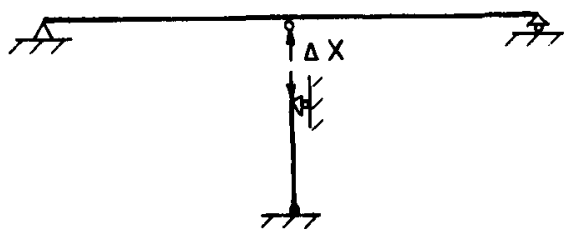

Bild 5.22: Ermittlung des Entfestigungsstabilitătsfaktors des Systems

Ein Inkrement der überzăhligen Grösse $\Delta X$ verursacht eine Durchbiegung des Trăgers

$$
\Delta W=\frac{\Delta X \cdot l^{3}}{6 \cdot E I T}
$$

und eine Stauchung der Stütze

$$
\Delta w=-\Delta x \cdot \frac{1-x_{1.1}}{D A}
$$

wobei $\chi_{1.1}=\frac{h D A}{E A}=$ Entfestigungsstabilitătsfaktor der axial beanspruchten Stütze

Der Entfestigungsstabilitătsfaktor des Systems ergibt sich zu

$$
x_{1}=\frac{\mathrm{DA} \cdot l^{3}}{6 \cdot \mathrm{ET} T \cdot\left(1-x_{1.1}\right)}=\frac{x_{1.1}}{1-x_{1.1}} \cdot \frac{l^{3}}{6 \cdot E T} \cdot \frac{E A}{h}=\frac{x_{1.1}}{1-x_{1.1}} \cdot \frac{w_{T}}{w_{S}}
$$

Die Grösse $w_{T}$ ist die Durchbiegung des Trägers infolge überzăhliger Grösse und $w_{S}$ ist die elastische Verkürzung der Stütze. Das Verhältnis $w_{T} / w_{S}$ ist im allgemeinen viel grösser als eins, so dass für gewöhnliche Stützenschlankheiten auch der Entfestigungsstabilitătsfaktor $\chi_{1}$ grösser als 1 ist. Dies bedeutet, dass mit dem Bruch der Stütze auch die Traglast des Systems erreicht wird. In einem 
solchen Fall wird die Traglast vom Eigenspannungszustand stark beeinflusst. Eine Bemessung der Stütze aufgrund der üblichen Methoden der Plastizitătstheorie ist somit problematisch.

Noch problematischer ist die Anwendung der üblichen Bemessungsmethoden, wenn die Stütze ausknickt (Bild 5.21c). Im allgemeinen erfolgt nach der Bildung eines plastischen Gelenkes die Entfestigungsstabilităt der Stütze (Bild 5.13).

Eine wesentliche Verbesserung des Tragverhaltens statisch unbestimmter Systeme, bei denen spröde und duktile Elemente wirken, kann erzielt werden, indem die spröden Elemente so bemessen werden, dass sie nie massgebend werden. Es stellt sich damit eine Bruchart ein, bei welcher die plastischen Verformungen in den duktilen Elementen stattfindet, so dass die Charakteristik des Gesamtsystems duktil wird. Im Abschnitt 5.5 wird die Untersuchung und Bemessung solcher Systeme mit der Plastizitătstheorie dargelegt.

\subsection{Systeme mit plastischen Verformungen in den Schubgelenken}

Wie im Abschnitt 4.4 beschrieben, erfolgt in einem Schubgelenk eine vertikale Verschiebung ohne Rotation (reines Schubgelenk) nur, wenn der Beton des Steges versagt. Wăhrend eines solchen Bruchvorgangs kann nur sehr wenig Energie dissipiert werden. Das bedeutet, dass bei statisch unbestimmten Systemen, z.B. einem System gemăss Bild 5.23, nach dem Erreichen der Schubfestigkeit (Betonfestigkeit im Steg) die Last abnehmen muss. Bei mehrach statisch unbestimmten Systemen kann eventuell die Last wieder erhöht werden, nachdem im Schubgelenk kein Widerstand mehr vorhanden ist (horizontales Plateau im Diagramm $V(w)$ mit $V=0$ ).
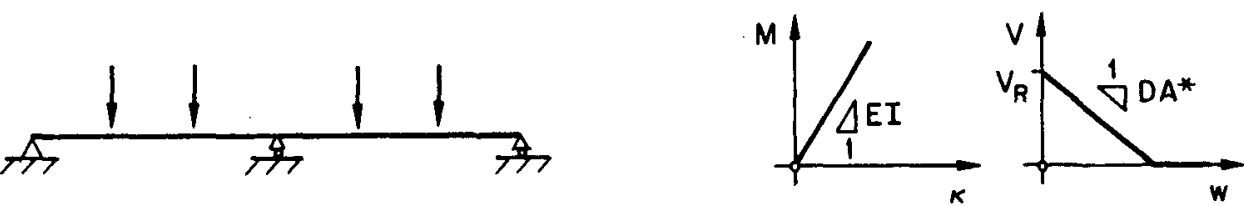

(a)

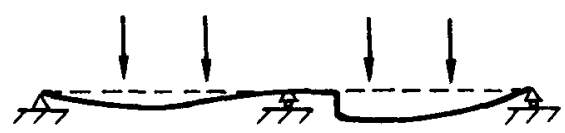

(b)

Bild 5.23: Statisch unbestimmtes System und plastische Verformung im Schubgelenk 


\subsection{Plastische Bemessung statisch unbestimmter Systeme mit sproden Elementen}

Duktile Elemente eines statisch unbestimmten Systems können mit der Plastizitătstheorie so bemessen werden, dass ein möglichst grosser Anteil der Lasten von den parallel gekoppelten Elementen aufgenommen wird. Die derart ermittelte Beanspruchung stellt eine untere Schranke dar. Als Beispiel wird der bereits untersuchte zweifeldrige Trăger betrachtet (Bild 5.24a). Wenn sich die Stütze duktil verhält, kann der erforderliche Stützenwiderstand mit den im Bild 5.24 dargestelten Mechanismen hergeleitet werden. Mit diesen Mechanismen wird berücksichtigt, dass ein möglichst grosser Lastanteil vom Trăger bis zu den Auflagern in A und $A^{\prime}$ abgetragen wird. Die so ermittelte kleinstmögliche Stützennormalkraft ist im Bild 5.24e als Funktion der Last $Q$ und des Trăgerwiderstandes $\mathrm{M}_{\mathrm{R}}^{+}$graphisch ausgewertet.

Im Abschnitt 5.3 wurde jedoch gezeigt, dass derartige Stützen im allgemeinen nicht genügend duktil sind, um eine Umlagerung der inneren Krăfte zwischen Druckglied und Trăger zu gewăhrleisten. Der Bruch der Stütze verursacht in diesem Fall den Kollaps des Systems. Um dies zu verhindem, muss die Stütze so bemessen werden, dass deren Beanspruchung nie den Widerstand erreicht. Dies wird üblicherweise erzielt, indem das System elastisch untersucht wird und indem die möglichen Zwângungen berücksichtigt werden. Diese Untersuchung ergibt im vorliegenden Fall:

$$
-N_{R}=\frac{8}{3} \cdot Q+3 \cdot k_{z} \cdot \frac{\text { ㅌl }}{l}
$$

wobei eine Vorkrümmung $\kappa_{z}$ des Trăgers als Zwăngung und die Stütze als starr betrachtet wird. Die Zwăngung ist jedoch eine Grösse, die von zahlreichen unbekannten Einflüssen abhängig ist, so dass eine solche Bemessungsmethode mit vielen Fragezeichen behaftet sein muss.

Ausser einer unteren Schranke der Stützennormalkraft existiert auch eine obere Schranke der Beanspruchung, die ebenfalls mit der Plastizitătstheorie bestimmt werden kann. Sie wird ermittelt, indem angenommen wird, die parallel gekoppelten Elemente wirkten gegen das zu bemessende spröde Element. Die Beanspruchung des spröden Elementes wird als äussere Last eingeführt, welche gegen das restliche System wirkt (Bilder 5.25 a,b,c). Die graphische Darstellung der zwei Schranken als Funktion der Last kann als Fliessfigur Last-Stützenbeanspruchung betrachtet werden. Die in den Bildem $5.25 \mathrm{~d}$ und e dargestellten Fliesstiguren zeigen, dass eine auf der oberen Schranke der Beanspruchung basierende Bemessung des Druckgliedes nicht zu einer zu grossen Ueberdimensionierung führt, wenn die Tragreserven des Trägers möglichst voll ausgenützt sind (obere Ecke der Fliessfigur). Wie schon enwăhnt, ermöglicht eine derartige Bemessung eine wesentliche Verbesserung des Systemverhaltens. Der Kollaps entspricht einem Mechanismus mit Biegegelenken.

Bild 5.26 zeigt die Anwendung dieser Methode bei der Ermittlung der Grenzwerte der Querkraft des im Abschnitt 5.4 beschriebenen Systems. Da diese Querkraft der Hălfte der Stützenkraft des vorangehenden Beispiels entspricht, sind auch die beiden Fliessfiguren vergleichbar.

Aehnliche Ueberlegungen werden heute schon in der Bodenmechanik bei der Bemessung von Stützmauern angestellt. Wie Bild 5.27 zeigt, können die untere und die obere Schranke der Belastung auf die Stützmauer (aktiver und passiver Erddruck) mit der Plastizitătstheorie ermittelt werden. Analog wie bei den untersuchten Systemen wird die untere Schranke mit einem Mechanismus, bei dem die Stützmauer versagt, bestimmt. Bei der oberen Schranke wird hingegen ein Mechanismus untersucht, bei dem die Stützmauer so gegen das Bodenmaterial wirkt, dass dort Versagen verursacht wird. 


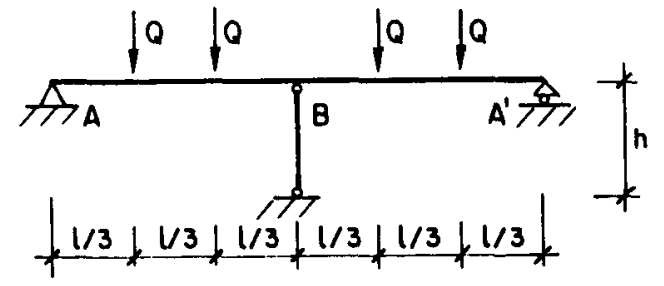

(a)

(b) Mechanismus 1 hी

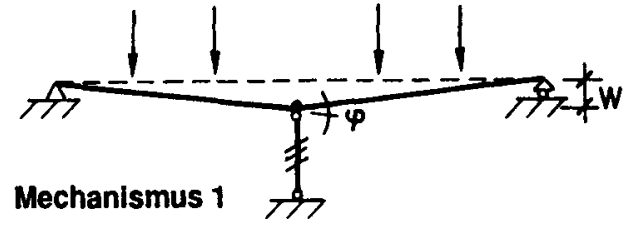

(c) Mechanismus 2 ,

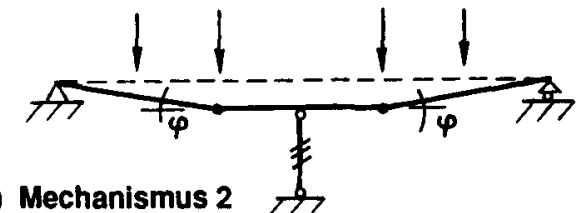

(d) Mechanismus 3 韦
Verhalten des Trägers

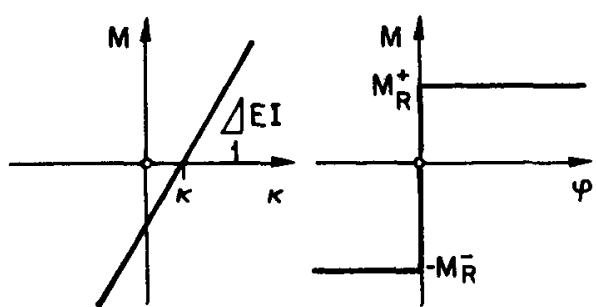

Verhalten der Stütze

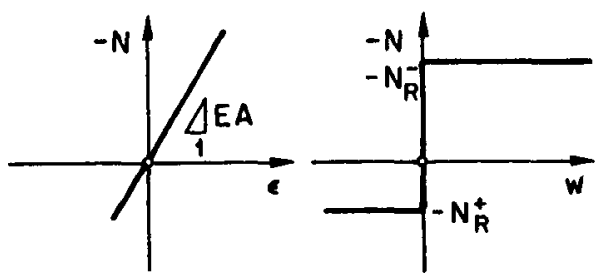

innere Arbeit : $A_{i}=-M_{R}^{+} \cdot \varphi+N_{R} \cdot w$

kin. Verträglichkeit : $\quad w=\frac{\varphi}{2} \cdot 1$

äussere Arbeit : $A_{a}=2 Q \cdot w$

erforderlicher $N_{A}:-N_{R} \geq 2 Q-\frac{2 M_{B}^{+}}{1}$

innere Arbeit : $A_{1}=-2 M_{A}^{+} \cdot \varphi+N_{A} \cdot W$

kin. Verträglichkeit : $\quad w=\frac{2}{3} \varphi \cdot 1$

äussere Arbeit : $A_{a}=3 Q \cdot W$

erforderlicher $N_{R}:-N_{R} \geq 3 Q-\frac{3 M_{A}^{+}}{I}$

innere Arbeit : $A_{i}=-2 M_{B}^{+} \cdot \varphi+N_{R} \cdot W$

kin. Verträglichkeit : $\quad w=\frac{1}{3} \varphi \cdot 1$

äussere Arbeit : $A_{a}=4 Q \cdot w$

erforderlicher $N_{R}:-N_{R} \geq 4 Q-\frac{6 M_{A}^{+}}{1}$

(e)

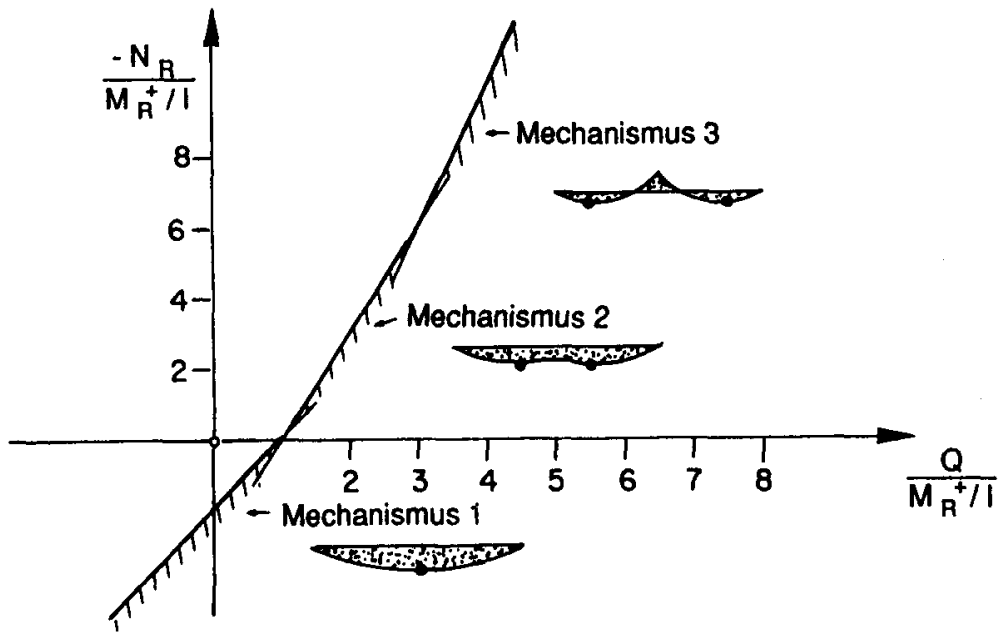

Bild 5.24: (a) System und Materialverhalten; (b) bis (d) Mechanismen für die Ermittlung der kleinstmöglichen Stützenbeanspruchung; $(e)$ unterer Grenzwert der Stützenbeanspruchung. 


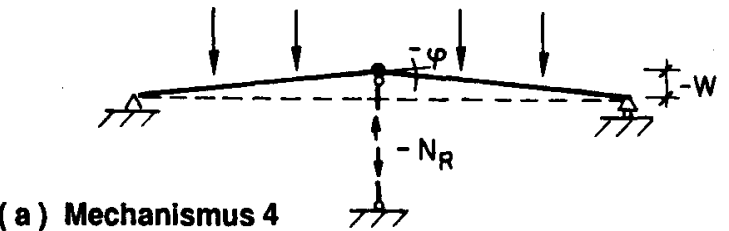

(a) Mechanismus 4

(b) Mechanismus 5

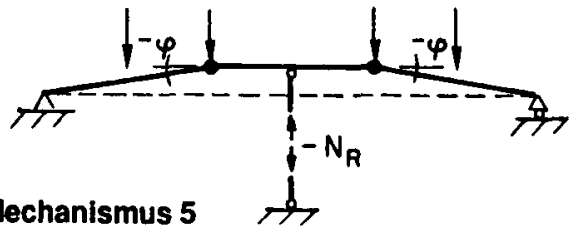

(c) Mechanismus 6

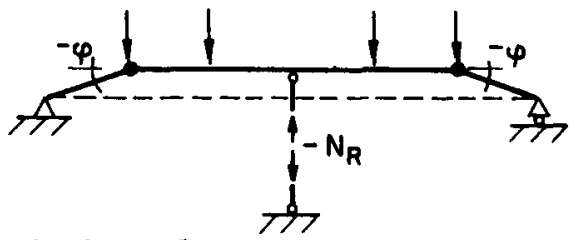

innere Arbeit $\quad: \quad A_{i}=-M_{\bar{A}} \cdot \varphi$

kin. Verträglichkeit : $\quad w=\frac{\varphi}{2} \cdot 1$

äussere Arbeit : $\quad A_{a}=2 Q \cdot w+N_{R} \cdot w$ maximaler $N_{R}:-N_{R} \leq 2 Q-\frac{2 M_{R}}{I}$

innere Arbeit : $\quad A_{i}=-2 M_{\bar{A}} \cdot \varphi$

kin. Verträglichkeit : $\quad w=\frac{2}{3} \varphi \cdot 1$

äussere Arbeit : $\quad A_{a}=3 Q \cdot w+N_{R} \cdot w$ maximaler $N_{R}:-N_{R} \leq 3 Q-\frac{3 M_{H}^{-}}{1}$

innere Arbeit : $A_{i}=-2 M_{\bar{B}}^{-} \cdot \varphi$

kin. Verträglichkeit : $\quad w=\frac{1}{3} \varphi \cdot 1$

äussere Arbeit : $\quad A_{a}=4 Q \cdot W+N_{R} \cdot W$ maximaler $N_{R}:-N_{R} \leq 4 Q-6 M_{R}^{-}$

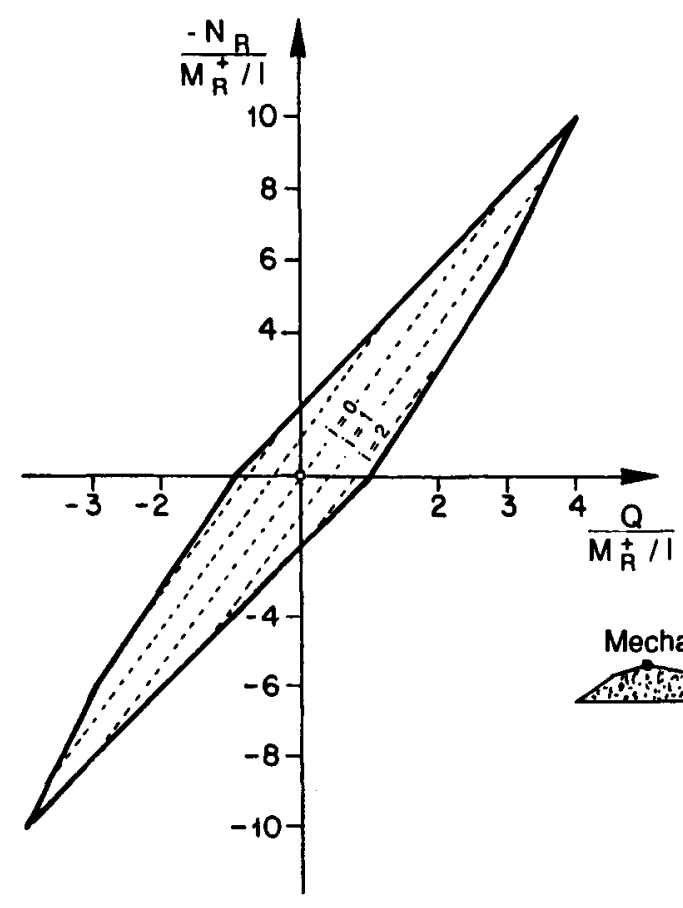

(d) $M_{A}^{-}=-M_{A}^{+}$ $\kappa_{z}=\frac{i \cdot M_{\mathrm{P}^{+}}}{6 \mathrm{EI}}$

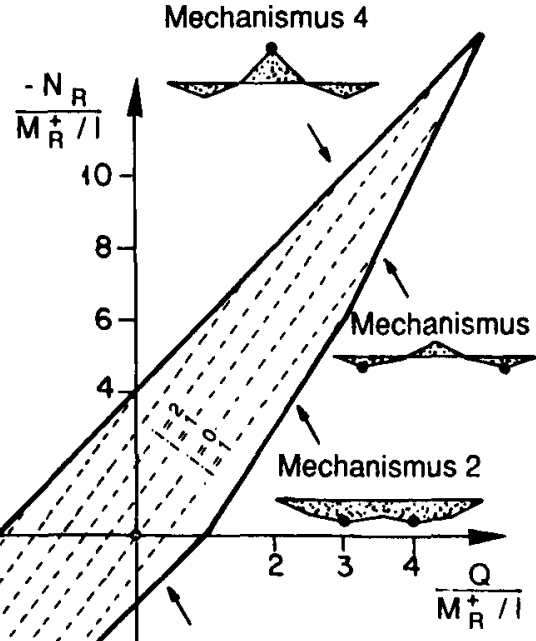

Mechanismus 1

risision

Bild 5.25: (a) bis c) Mechanismen für die Ermittlung der grösstmöglichen Stützenbeanspruchung; (d) und (e) Fliessfigur Last-Stützenbeanspruchung. 

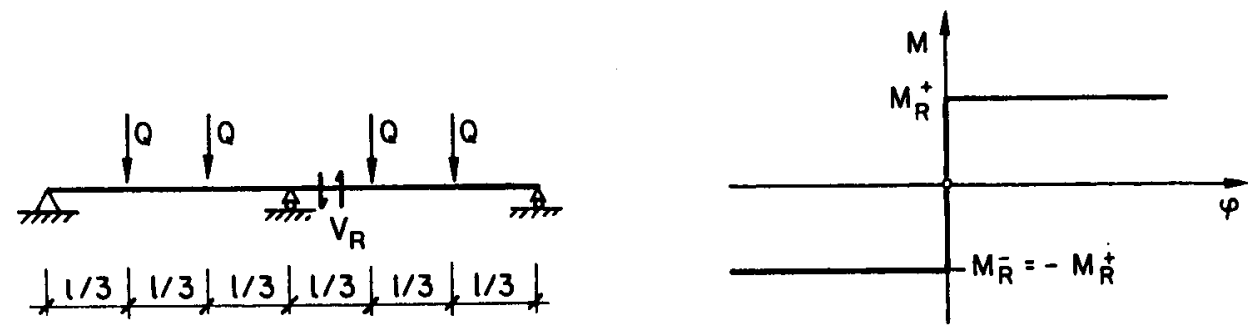

(a)

(b)

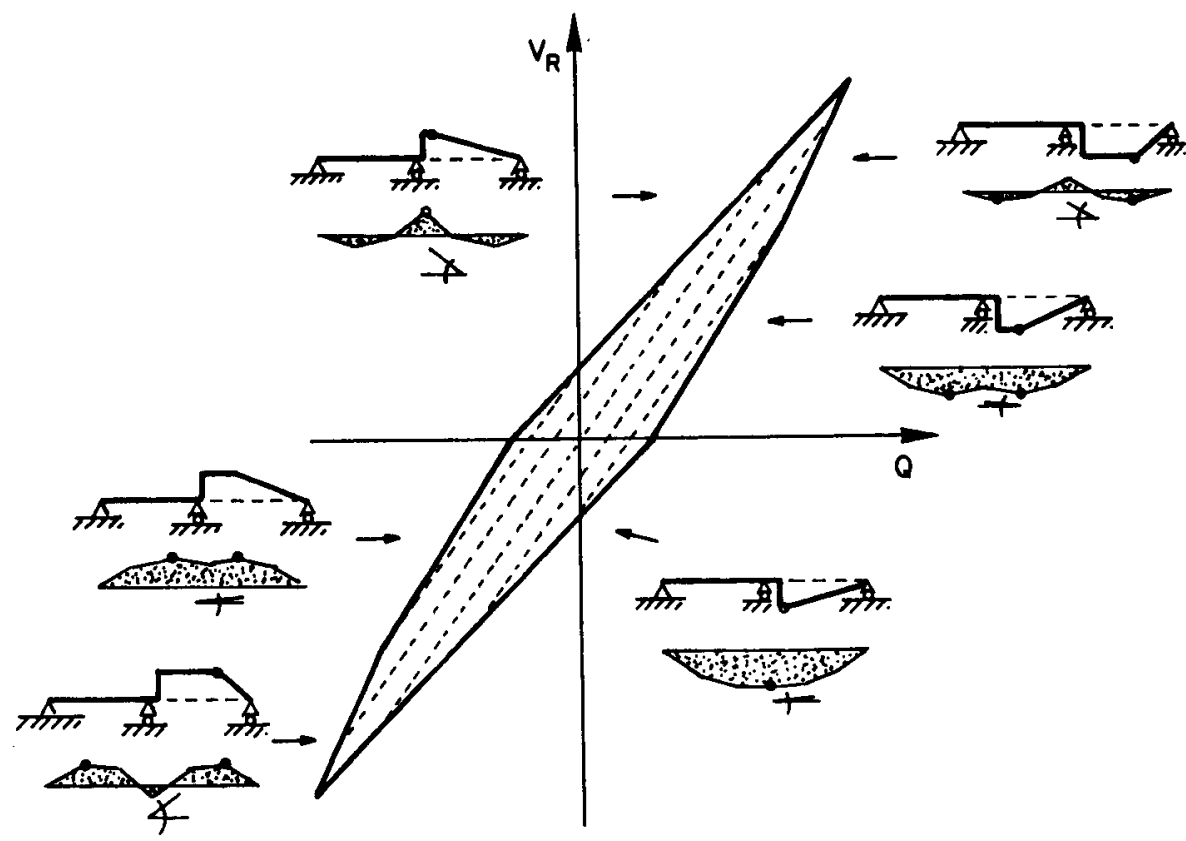

Bild 5.26: Grenzwerte der Schubbeanspruchung eines statisch unbestimmten Systems

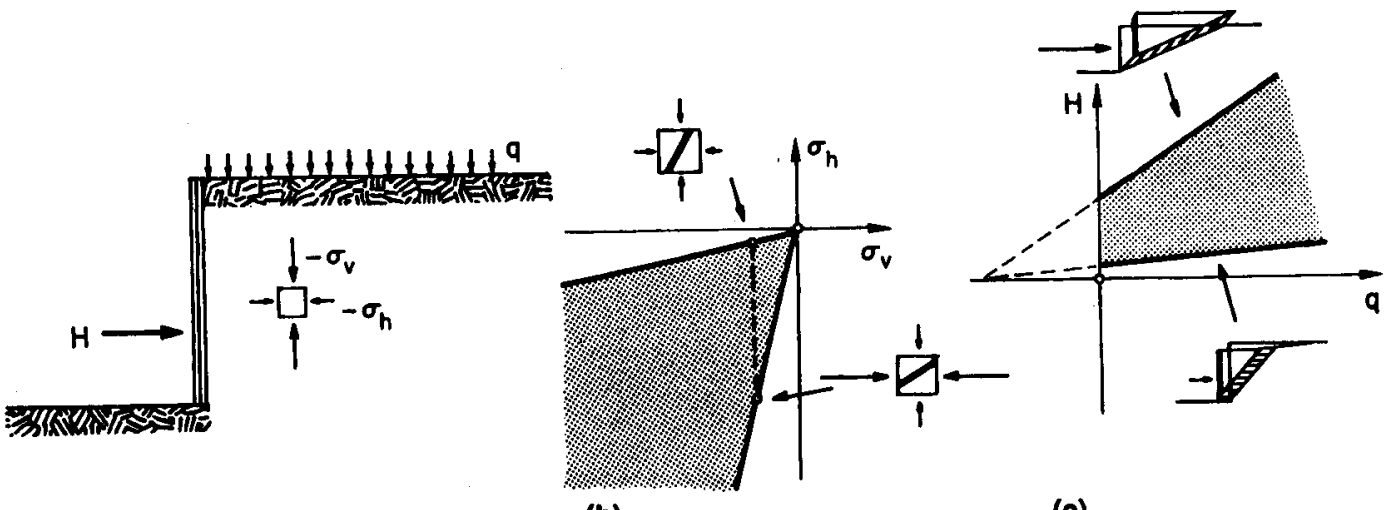

(a)

(b)

(c)

Bild 5.27: (a) Erddruck auf eine Stützmauer; (b) und c) Fliessfigur für ein Bodenelement und für die Stützmauer. 


\section{Einschränkung der Umlagerung durch breite Risse}

\subsection{Elnführung}

Im Abschnitt 4.2 wurde gezeigt, dass die Fortpflanzung von Lăngsrissen in die unbewehrte Druckzone eines Biegebalkens die Festigkeit und die Duktilităt beeintrăchtigen können. In solchen Fällen kann die nach der Plastizitätstheorie ermittelte Traglast in Wirklichkeit nicht erreicht werden. Der Grund dafür ist die durch ungenügende Verzahnung der Rissufer verhinderte Kraftübertragung (siehe Abschnitt 3.2, Bild 3.15). Im vorliegenden Abschnitt werden weitere Beispiele behandelt, bei denen sich die Tragwirkung, aus welcher nach Plastizitătstheorie die Traglast bestimmt wird, nicht einstellen kann. Es handelt sich dabei um schubbeanspruchte Balken, Stützen und Platten ohne Schubbewehrung. Aehnliche Tragwerke, jedoch mit Schubbewehrung, wurden im Abschnitt 4.4 behandelt. Dabei wurde gezeigt, dass bei diesen Tragwerken mit der Anordnung einer ausreichenden Bewehrung die Traglast nach Plastizitătstheorie richtig bestimmt werden kann, und dass sich die zugehörigen Spannungsfelder (Tragwirkungen) effektiv einstellen können.

\subsection{Schubbeanspruchte Balken ohne Baggelbewehrung}

Als Beispiel wird zuerst der im Bild 6.1 dargestelte einfache Balken mit konstanter Trăgerhöhe behandelt. Die Bewehrung besteht aus Lăngsbewehrungsstăben, die hinter den Auflagem voll verankert sind.

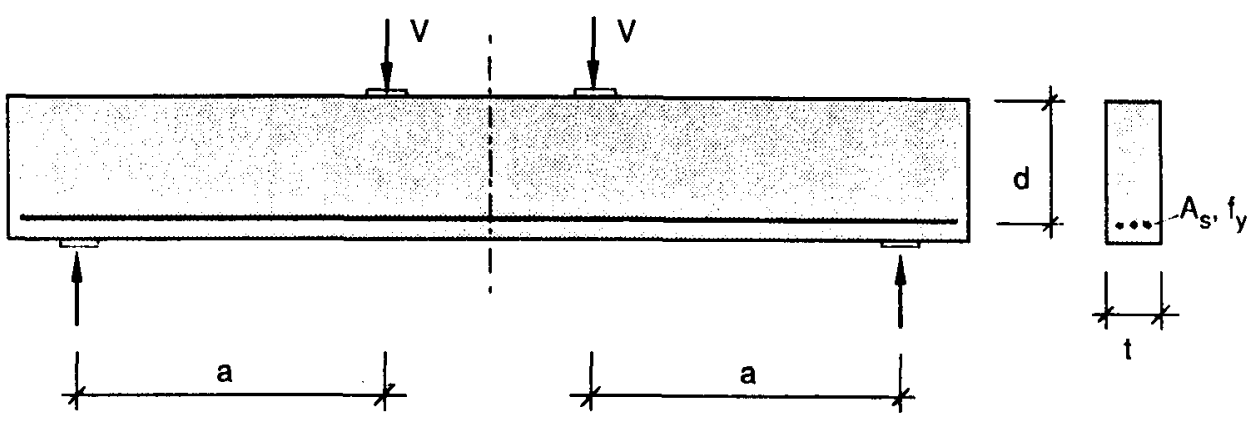

Bild 6.1: Einfacher Balken ohne Bügelbewehrung

Zuerst wird die Lösung nach der Plastizitătstheorie entwickelt. Ohne Ausnützung der Betonzugfestigkeit und ohne Schubbewehnung ist die sogenannte direkte Abstützung die einzige mögliche Tragwirkung. Die Last stützt sich direkt gegen das Auflager ab. Zwischen der Last und der Auflagerkraft bildet sich somit eine auf Druck beanspruchte Betonstrebe aus. Bild 6.2a zeigt das von Drucker (1961) vorgeschlagene Spannungsfeld für die Beschreibung dieser Tragwirkung. Die Gleichgewichtsbedingungen sind überall erfült, und die Betonfestigkeit sowie die Stahlfestigkeit werden nirgends überschritten. Das gezeigte Spannungsfeld ist somit eine Lösung nach dem unteren Grenzwertsatz der Plastizitătstheorie. Im Bild $6.2 \mathrm{~b}$ sind die Wirkungslinien der Spannungsresultierenden dargestellt. 


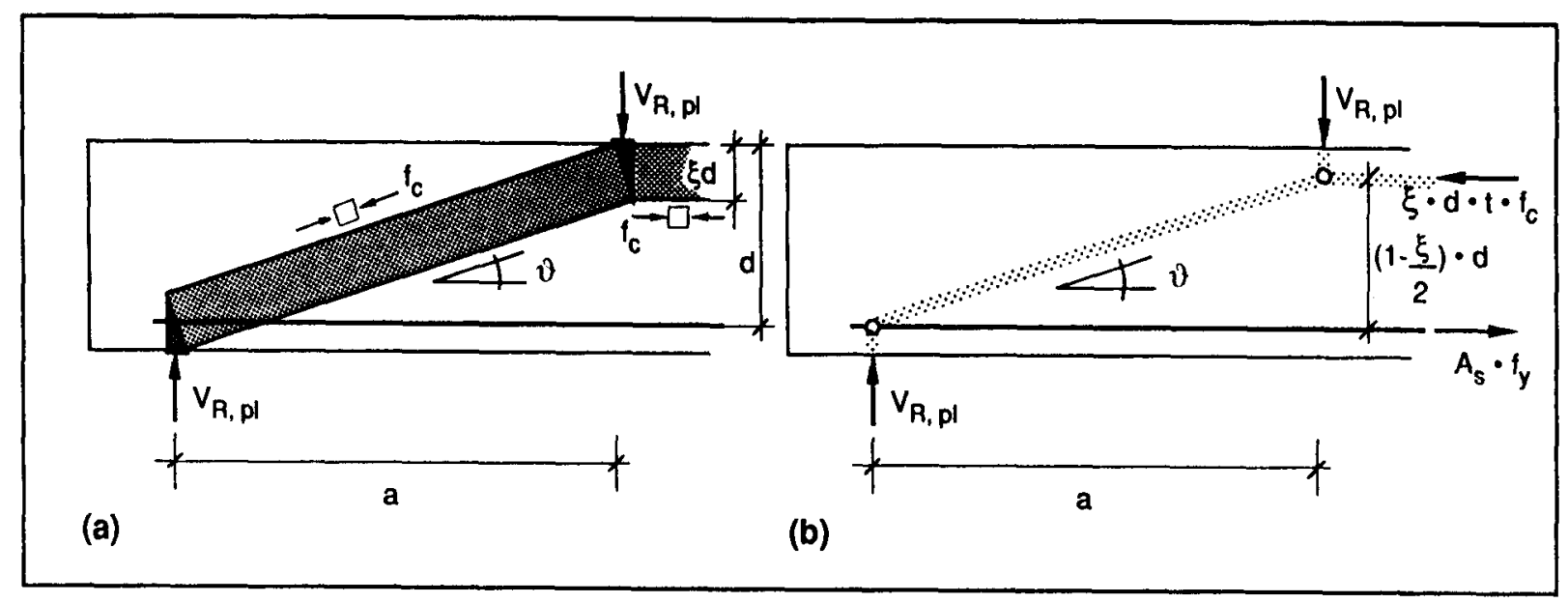

Bild 6.2: Lősung aufgrund des unteren Grenzwertsatzes der Plastizitătstheorie: (a) Spannungsfeld; (b) Wirkungslinien der Spannungsresultierenden.

Daraus lassen sich die Druckstrebenneigung $\vartheta$ und die Last im Bruchzustand $\mathrm{V}_{\mathrm{R}, \mathrm{pl}}$ als Funktion der Balkenabmessungen und der Materialfestigkeiten ermittein:

$$
\begin{aligned}
& \operatorname{cot\vartheta }=\frac{a}{d \cdot\left(1-\frac{\xi}{2}\right)} \\
& \mathrm{V}_{\mathrm{R}, \mathrm{pl}}=\frac{\xi \cdot d \cdot t \cdot f \mathrm{c}}{\cot \vartheta}=\xi \cdot \frac{d^{2}}{\mathrm{a}} \cdot t \cdot t_{c} \cdot\left(1-\frac{\xi}{2}\right)
\end{aligned}
$$

mit $\quad \xi=\omega=\frac{A_{S} \cdot f y}{d \cdot t \cdot f}$

Die zweite Gleichung lässt sich auch aus dem Vergleich des Momentenwiderstandes mit der Momentenbeanspruchung im mittleren Bereich bestimmen. Die beschriebene Lösung nach dem unteren Grenzwertsatz ist gleichzeitig auch eine vollstândige Lơsung nach der Plastizitătstheorie. Marti (1980) und Jensen (1981) haben 'gezeigt, dass eine Lơsung nach dem oberen Grenzwertsatz der Plastizitătstheorie (Mechanismusmethode) die gleiche Traglast ergibt.

Das beschriebene Spannungsfeld kann aber unter Umständen von der sich effektiv einstellenden Tragwirkung betrăchtlich abweichen. Mit der Untersuchung dieser Abweichung kann erklărt werden, warum die plastizitătstheoretische Traglast in Wirklichkeit zum Teil nicht erreicht werden kann. Während einer monotonen Erhöhung der Last $V$ von null bis zum Erreichen der Bruchlast $V_{R}$ lassen sich mehrere Phasen mit den entsprechenden Tragwirkungen erkennen.

Im ungerissenen Zustand kann - abgesehen vom Eigenspannungszustand - das Spannungsfeld mit der Elastizitätstheorie ermittelt werden. Die Hauptspannungstrajektorien eines solchen Spannungsfeldes zeigt Bild 6.3. 


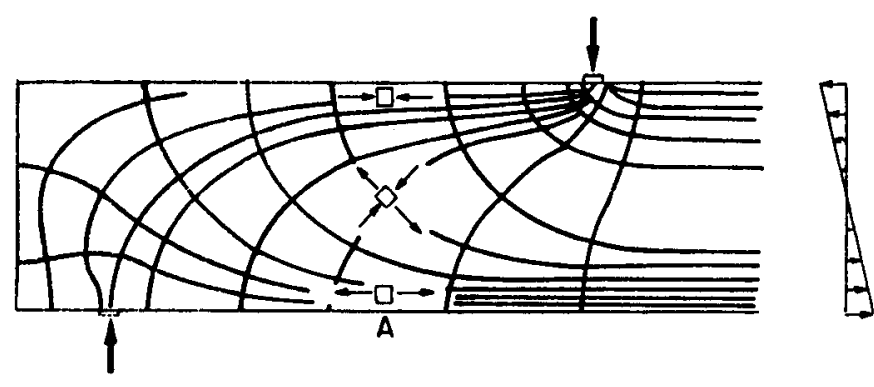

Bild 6.3: Spannungsfeld im ungerissenen Zustand nach der Elastizitătstheorie
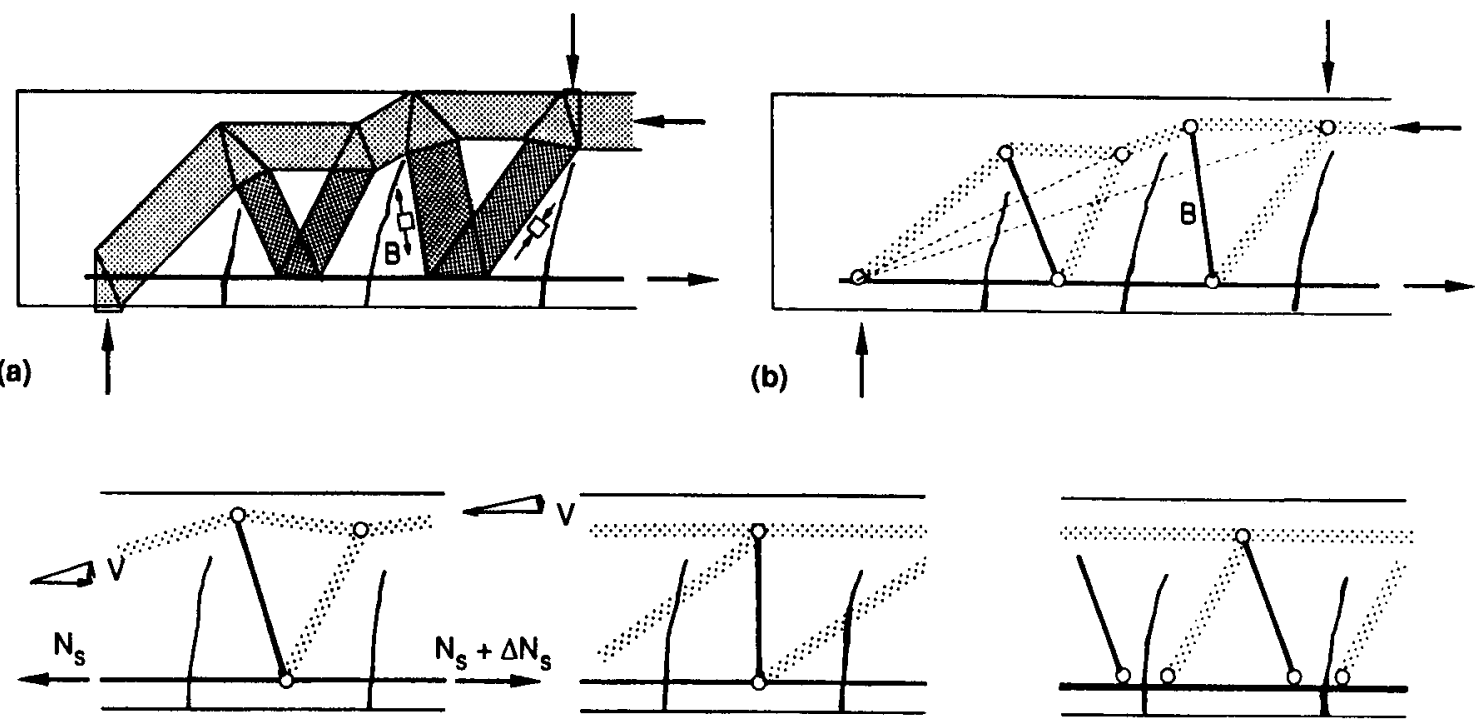

(c) "Konsolenwirkung "

(d) "Verzahnungswirkung"

(e) "Dübelwirkung "

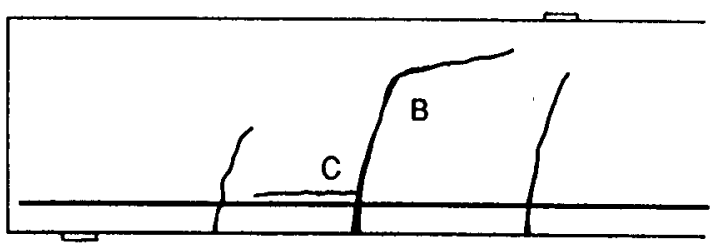

(f)

Bild 6.4: Tragwirkung ohne Kraftübertragung über die Biegerisse: (a) Darstellung mit einem Spannungsfeld; (b) und (c) mit den zugehörigen Resultierenden; (d) und (e) weitere Tragwirkungen in diesem Zustand; (f) Fortpflanzung der Biegerisse. 
Bekanntlich bilden sich die ersten Risse in der Zugzone, d.h. die Zugfestigkeit wird zuerst in A erreicht. Nachdem Biegerisse entstanden sind, ist die im Bild 6.3 beschriebene Tragwirkung nicht mehr wirksam. Nimmt man an, dass über diese Risse keine Kraftübertragung erfolgt, kann die neue innere Tragwirkung mit dem im Bild 6.4a dargestellten Spannungsfeld (Bild 6.4b zeigt die zugehörigen Resultierenden) beschrieben werden. Diese Tragwirkung ist schon von Kani (1955) erkannt und wie folgt beschrieben worden: "Durch die Querrisse wird die Zugzone in einzelne Betonkörper aufgeteilt, die man sich als oben in die Druckzone eingespannte Kragbalken vorstellen kann."

Diese Konsolen sind durch die Aenderung der Lăngsbewehrungskraft belastet (Bild 6.4c). Weitere mögliche Tragwirkungen sind in den Bildern 6.4d und 6.4e schematisch mit den Resultierenden dargestellt. Beim Tragmodell im Bild 6.4d werden Betondruckkräfte auch über die Risse übertragen. Das ist dank der Rauhigkeit der Rissufer (Verzahnung) möglich. Bild 6.4e zeigt eine Tragwirkung, bei welcher die Lăngsbewehrung auch Krăfte in Querrichtung übertrăgt (sogenannte Dübelwirkung). Das effektiv vorhandene Spannungsfeld resultiert aus der Kombination der drei erwăhnten Tragwirkungen wie ebenfalls Hamadi, Regan (1980) sowie auch Reineck (1982) und andere gezeigt haben.

Bei weiterer Erhöhung der Last $V$ wird die Zugfestigkeit im Bereich $B$ (Bild 6.4a) erreicht. Der ursprünglich fast vertikal verlaufende Biegeriss pflanzt sich in mehr oder weniger horizontaler Richtung fort. Ein typischer Rissverlauf ist im Bild $6.4 f$ angegeben. Die Fortpflanzung dieses Risses ist verbunden mit einem Kollaps der drei erwăhnten Tragwirkungen. Die "Konsolenwirkung" und die "Verzahnungswirkung" verlieren ihre Tragfăhigkeit, weil die Betonzugbänder bei B gebrochen sind. Auch die "Dübelwirkung" ist abgeklungen, weil sich ein Riss bei $C$ (Bild 6.4f) gebildet hat.

Die neue Tragwirkung, die sich einstellen könnte, ist die schon enwähnte Lösung nach der Plastizitătstheorie, d.h. die sogenannte Direktabstützung. Bild 6.5a zeigt das Rissbild eines am Institut für Baustatik und Konstruktion der ETH Zürich durchgeführten Schubversuchs knapp vor dem Bruch. In diesem Bild ist das Spannungsfeld zur Beschreibung der Direktabstützung eingetragen. Die Abmessung der Druckstrebe (Hőhe der Druckzone $x=\xi \cdot d$ ) wurde mit der folgenden Gleichung ermittelt:

$$
\xi=1-\sqrt{1-\frac{2 \cdot V_{R} \cdot a}{t \cdot d^{2} \cdot t} c}
$$

wobei $V_{R}$ die effektiv beobachtete Bruchlast und nicht die nach der Plastizitätstheorie ermittelte Traglast $V_{R, p l}$ ist. Da $V_{R}$ kleiner als $V_{R, p l}$ ist (die Bewehrung ist nicht geflossen), ist auch $\xi$ kleiner als $\omega$. Aus Bild 6.5a ist ersichtlich, dass der Hauptriss durch die theoretische Druckstrebe verlăuft. Die Móglichkeit einer Kraftübertragung über den Riss ist von den relativen Verschiebungen der Rissufer und von der Rauhigkeit der Rissoberflăche abhăngig (siehe Bild 3.15). Die wăhrend des Versuchs gemessenen relativen Verschiebungsvektoren sind für zwei Laststufen $V=0.87 \cdot V_{R}$ und $\left.V=0.95 \cdot V_{R}\right)$ im Bild $6.5 b$ dargestellt. 


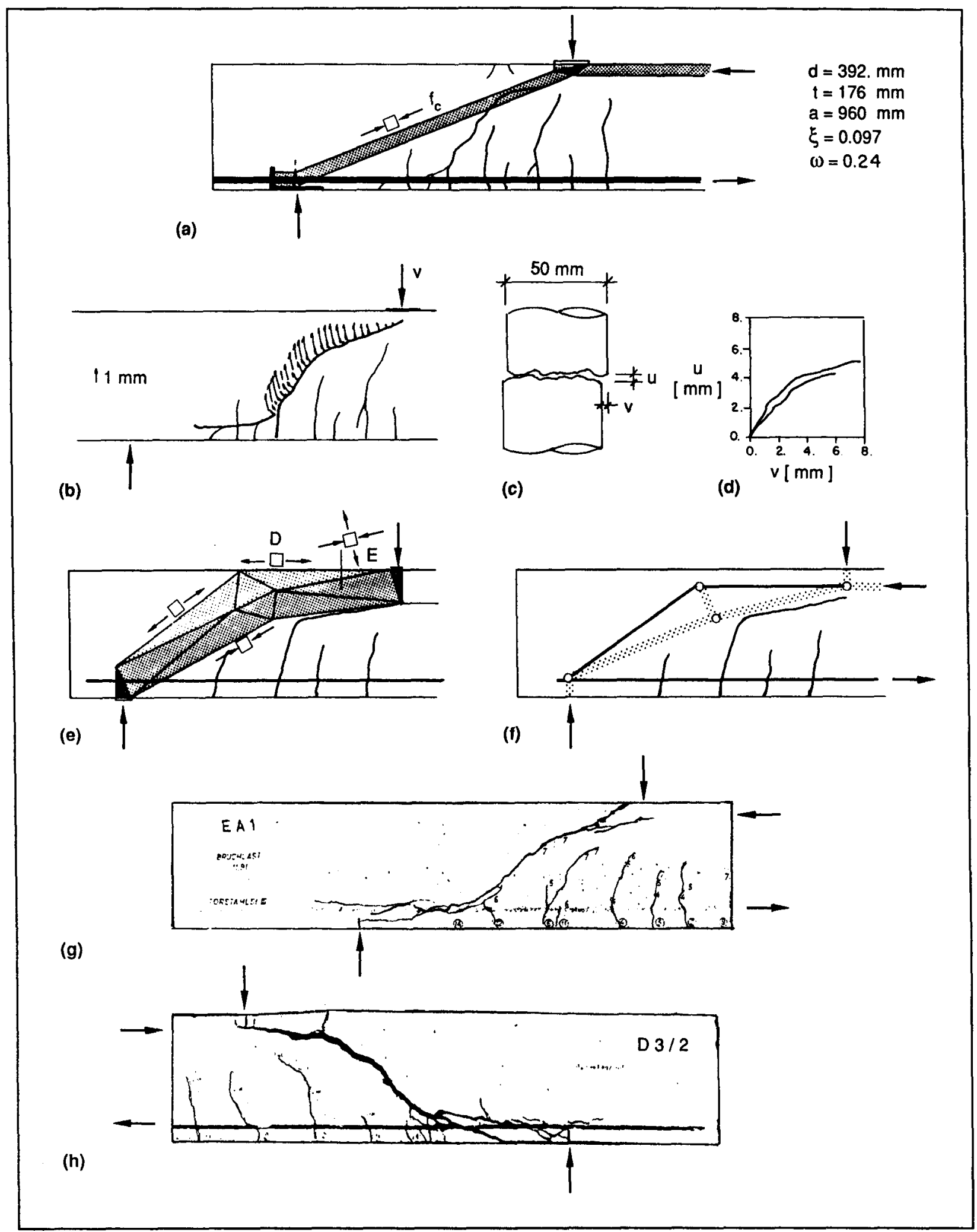

Bild 6.5: (a) Rissbild im Bruchzustand mit dem Spannungsfeld zur Beschreibung der Direktabstützung; (b) gemessene Relatiwerschiebung des Hauptrisses; (d) Beziehung zwischen Rissöffnung und Rissschiebung, bei der keine Kraftübertragung erfolgt, gemessen an einer aus einem Versuchsbalken entnommenen Probe; (e) Spannungsfeld und (f) Verlauf der Resultierenden zur Beschreibung der Tragwirkung, wenn keine Kraftübertragung im Hauptriss erfolgt; (g) und (h) zwei mögliche Brucharten. 


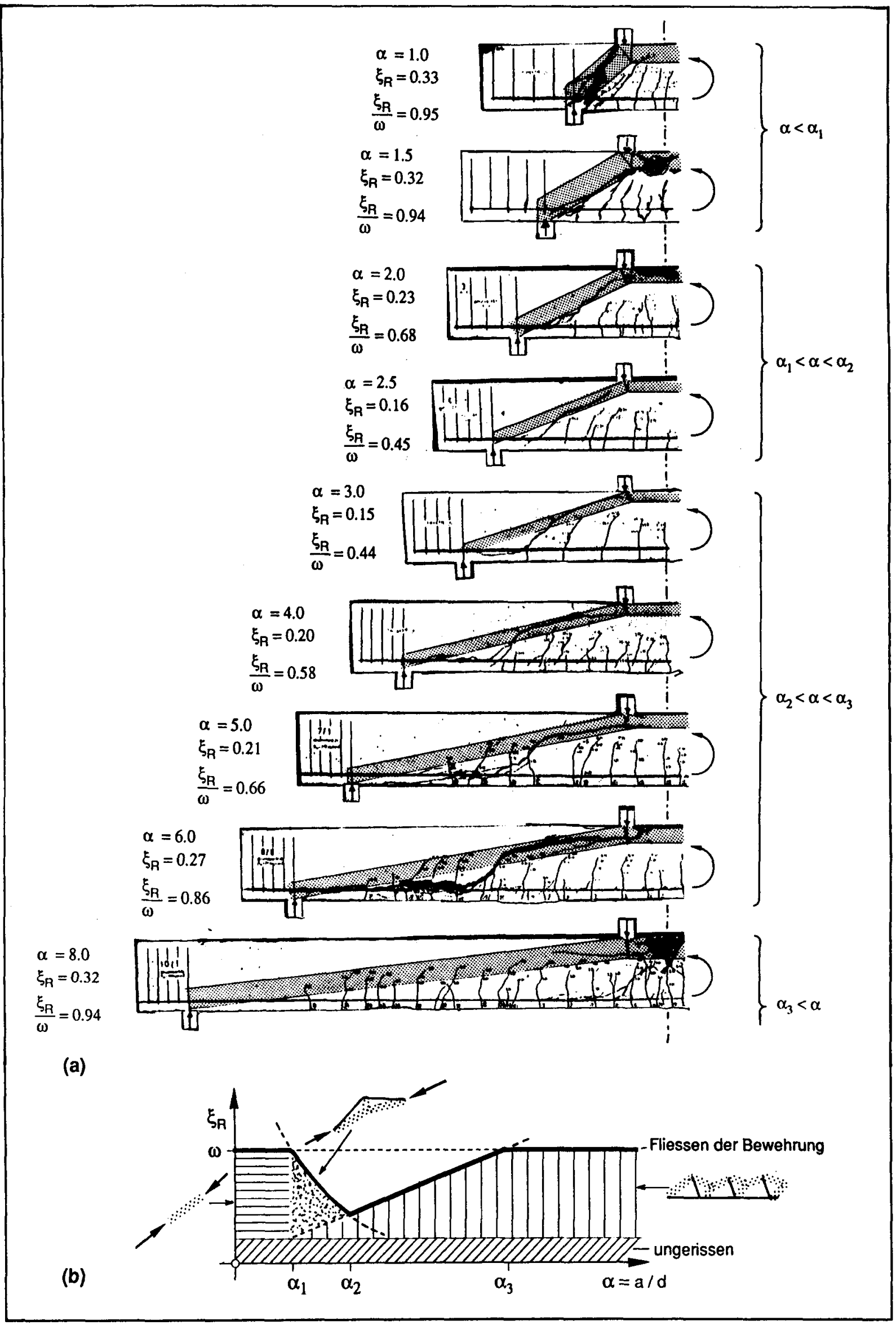

Bild 6.6: (a) Rissbild und direkte Druckstrebe von Balken verschiedener Schlankheit; (b) Einfluss auf die Traglast. 
Diese Verschiebungen sind mit den im Bild 3.15 angegebenen Beziehungen zwischen Rissőffnung u und Rissschiebung $v$ zu vergleichen. Diese Kurven zeigen die Verschiebungsvektoren, bei welchen kein Kontakt zwischen zwei Rissufern mehr besteht und somit auch keine Kraftübertragung erfolgt. Sie wurden an Proben gemessen (Bild 6.5c), die nach ihrer Entnahme aus dem Versuchsbalken in zwei Teile gebrochen worden waren. Dieselben Kurven sind im gleichen Massstab wie im Bild $6.5 \mathrm{~b}$ im Bild 6.5d angegeben. Ein Vergleich der beiden Bilder zeigt, dass im Riss des Versuchbalkens praktisch keine Kraftübertragung mehr stattgefunden hatte.

Ein Spannungsfeld mit einer zwischen Last und Auflagerkraft direkt verlaufenden Betonstrebe kann sich somit nicht einstellen. Eine solche Druckstrebe ist nur zulăssig, wenn sie im Mittelbereich umgelenkt wird. Bild 6.5e zeigt ein solches Spannungsfeld. Die Betondruckstrebe wirkt zusammen mit einem Betonzugband, das ebenfalls im Mittelbereich einen Knick aufweist, damit sich die Umlenkkrätte gegenseitig kompensieren können.

Der Bruch tritt schliesslich ein, weil das Zugfeld im Bereich D reisst, oder weil die Festigkeit in der auf Druck und Zug beanspruchten Zone $E$ erreicht wird. Für eine Ueberbeanspruchung dieser Zone kann auch der Riss, der sich bis zur Lasteinleitungszone fortpflanzt, verantwortlich sein. Die in den Bildern $6.5 \mathrm{~g}$ und $6.5 \mathrm{~h}$ dargestelten Versuche von Leonhardt, Walther (1962a) zeigen diese beiden möglichen Brucharten.

Es ist leicht verständlich, dass die Traglast des im Bild 6.5e dargestellten Spannungsfeldes (geknickte Druckstrebe) kleiner ist als die nach der Plastizitătstheorie ermittelte Traglast (gerade Druckstrebe). Die Bruchlast ist somit vom Rissbild stark abhăngig. Bild 6.6 zeigt den Einfluss der Balkenschlankheit $\alpha=a / d$ auf das Rissbild und damit auf die Bruchlast. In den Rissbildern der von Leonhardt, Walther (1962a) durchgeführten Versuche sind die direkten Druckstreben eingezeichnet (Bild 6.6a). Bild 6.6b zeigt den qualitativen Verlauf der Höhe der Druckzone $\xi$ im Bruchzustand als Funktion der Schlankheit $\alpha$. Bei der Berechnung von $\xi$ wurde angenommen, dass die Druckstreben konstant mit $f_{c}$ beansprucht sind. Diese Grösse beschreibt indirekt auch die Bruchlast. Das Bild erlaubt einen direkten Vergleich der Plastizitătstheorie mit der Wirklichkeit. Nach der Plastizitătstheorie ist $\xi=\omega$ für $\omega \leq 0.5$.

Im Bild 6.6 lassen sich folgende Bereiche unterscheiden:

$\alpha \leq \alpha_{1}$ : Bei sehr kleinen Schlankheiten wird ein Teil der Last von Anfang an direkt aufgenommen. Aus diesem Grund bilden sich die Risse derart, dass die direkte Druckstrebe nicht gestört wird. Die Traglast nach der Plastizitătstheorie kann somit erreicht werden.

$\alpha_{1} \leq \alpha \leq \alpha_{2}$ : Die Bruchart ist in den Bildern $6.5 \mathrm{~g}$ und $\mathrm{h}$ beschrieben. Da die Festigkeit der geknickten Druckstrebe kleiner ist als diejenige der direkten Druckstrebe, wird die plastizitätstheoretische Traglast nicht erreicht. Je kleiner die Schlankheit, desto kleiner ist die Störung durch den Hauptriss. 
$\alpha_{2} \leq \alpha \leq \alpha_{3}$ : Für mittlere Schlankheiten muss die Druckstrebe einen so grossen Knick aufweisen, dass die Traglast des Systems sogar kleiner ist als die zur Rissfortpflanzung erforderliche Last. Mit zunehmender Schlankheit und ungefăhr konstanter Schubbeanspruchung nimmt die Biegebeanspruchung und somit auch die Abmessung der Druckstrebe zu.

$\alpha \geq \alpha_{3}$ : Bei grossen Schlankheiten fliesst die Lăngsbewehrung, bevor die Rissfortpflanzungslast erreicht wird. Obwohl die Traglast nach der Plastizitätstheorie erreicht wird, ist die effektiv vorhandene Tragwirkung (Bilder 6.4c, d, e) nicht vergleichbar mit der Direktabstützung.

Einen ăhnlichen Verlauf wie derjenige im Bild 6.6b ermittelte Kani (1964) experimentell. Es ist zu bemerken, dass eine kleine Abweichung des Rissverlaufs die Tragwirkung und somit die Bruchlast relativ stark beeinflussen kann. Damit resultiert vor allem bei kleinen Trăgern eine grosse Streuung der experimentell ermittelten Bruchlasten. Da das Rissbild auch vom Eigenspannungszustand und von der Belastungsgeschichte abhăngig ist, wird die Bruchlast auch von diesen Faktoren beeinflusst. Aus diesen Gründen wird auch eine theoretische Ermittlung der Bruchlast immer mit grossen Schwierigkeiten und Unsicherheiten behaftet sein.

Die Korrelation zwischen Rissbild und Bruchlast zeigt den Weg zu einer Verbesserung des Tragverhaltens und einer Erhöhung der Traglast auf, indem das Rissbild durch konstruktive Massnahmen gūnstig beeinflusst wird. Die Zone, in der sich die direkte Druckstrebe einstellen soll, kann zum Beispiel rissfrei gehalten werden, indem der Trăger so vorgespannt wird, dass sich die Last von Anfang an direkt abstützt. Dies erklärt, wanum vorgespannte Träger einen grösseren "Schubwiderstand" autweisen als schlaff bewehrte Trăger. Diesen Einfluss zeigt Bild 6.7, in welchem das Verhăltnis $V N_{\text {flex }}\left(V_{\text {flex }}\right.$ ist die Biegetraglast, d.h. die Traglast nach der Plastizitătstheorie) als Funktion des Verhăltnisses PN (Neigung der Druckresultierenden) von fünf ăhnlichen Versuchen von Sozen, Zwoyer, Siess (1959) dargestellt ist (Auswertung siehe Anhang A4).

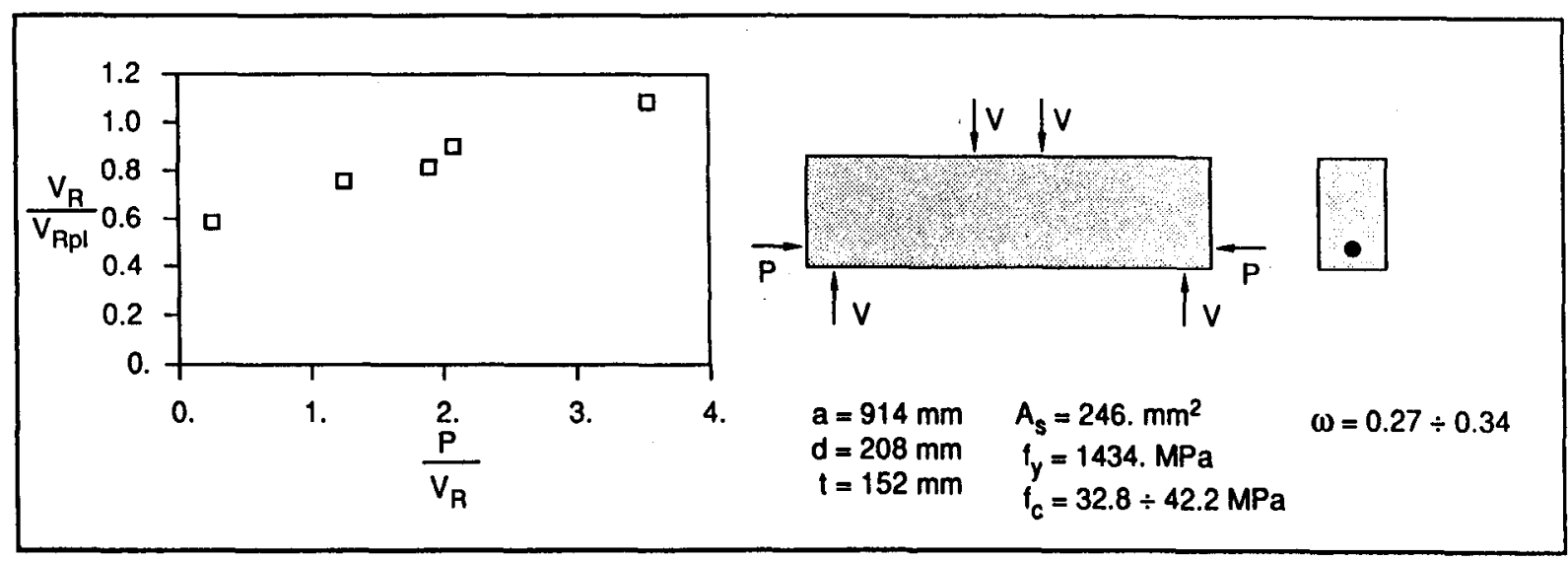

Bild 6.7: Verhăltnis Bruchlast/Traglast nach Plastizitătstheorie in Funktion des Verhăltnisses $\mathrm{P} N$ 
Eine ähnliche Verbessenung kann festgestellt werden, wenn die Bewehrung im schubbeanspruchten Bereich mit dem Beton nicht verbunden ist. Bild 6.8 zeigt die Rissbilder und die Spannungsfelder zweier Versuche, die sich nur in der Güte des Verbundes unterscheiden. Bei Trăgern mit Bewehrung ohne Verbund oder mit einer schlechten Verbundqualităt bilden sich Risse nur im mittleren Bereich. Wie im Bild 6.8b dargestellt, wird die geneigte Druckstrebe von diesen Rissen praktisch nicht beeinflusst, so dass eine viel höhere Bruchlast erreicht wird als bei Trăgern mit guten Verbundeigenschaften (Bild 6.8a).

(a)

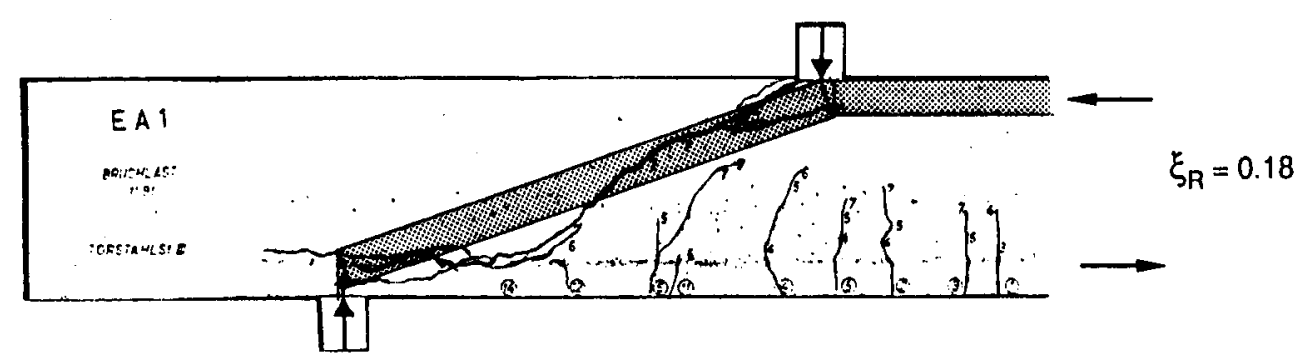

(b)

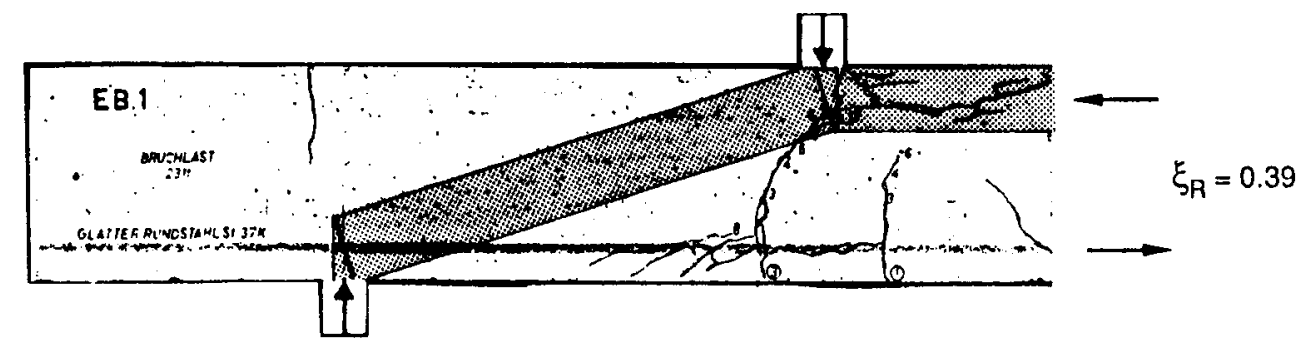

Bild 6.8: Rissbilder und Spannungsfelder (Direktabstützung) für ăhnliche Trăger mit unterschiedlichen Verbundeigenschatten: (a) guter Verbund; (b) schlechter Verbund; (Versuche von Leonhardt, Walther, 1962).

Auch die Balkengeometrie und der Verlauf der Bewehrung haben einen grossen Einfluss auf das Rissbild. Dies zeigt Bild 6.9, in welchem die von Mörsch (1929) beschriebenen Versuche dargestellt sind. Beim ersten Balken mit konstanter Trăgerhöhe wird die direkte Druckstrebe wegen den schlechten Verbundeigenschaften durch Risse praktisch nicht gestört. Da aber mit der Bildung der direkten Druckstrebe die volle Bewehrungskraft hinter dem Auflager verankert werden muss, tritt ein Verankerungsbruch ein, bevor die Fliessgrenze und die plastizitătstheoretische Traglast erreicht werden können. Beim zweiten Balken bewirkt die variable Trăgerhöhe die Entstehung eines breiten Risses im kritischen Bereich. Die Bruchlast ist somit kleiner als diejenige des Balkens mit konstanter Trägerhöhe. Beim dritten Balken kann sich ein ähnlicher Riss wie im zweiten Balken infolge der oberen Lăngsbewehrung nicht in die kritische Zone fortpflanzen. Die Bruchlast wird damit beträchtlich erhöht. Eine wesentliche Erhöhung der Bruchlast kann erzielt werden, indem die Druckstrebe im kritischen Bereich bewehrt wird. Bild 6.10 zeigt die Bewehrungsanordnung und das Rissbild im Bruchzustand eines von Muttoni, Thürlimann (1986) durchgeführten Versuchs. Breite Risse sind nur im unbewehrten Bereich entstanden, so dass sich die direkte Druckstrebe ausbilden konnte. Die Last konnte damit bis zum Fliessen der Lăngsbewehrung erhöht werden. Die Bruchlast war rund doppelt so gross wie diejenige des ăhnlichen Balkens ohne Druckstrebenbewehrung (Bild 6.5a). Mit der Anordnung einer üblichen Bügelbewehrung, die die ganze Trägerhöhe umfasst, kann sich die im Abschnitt 4.4 beschriebene indirekte Abstützung einstellen. 


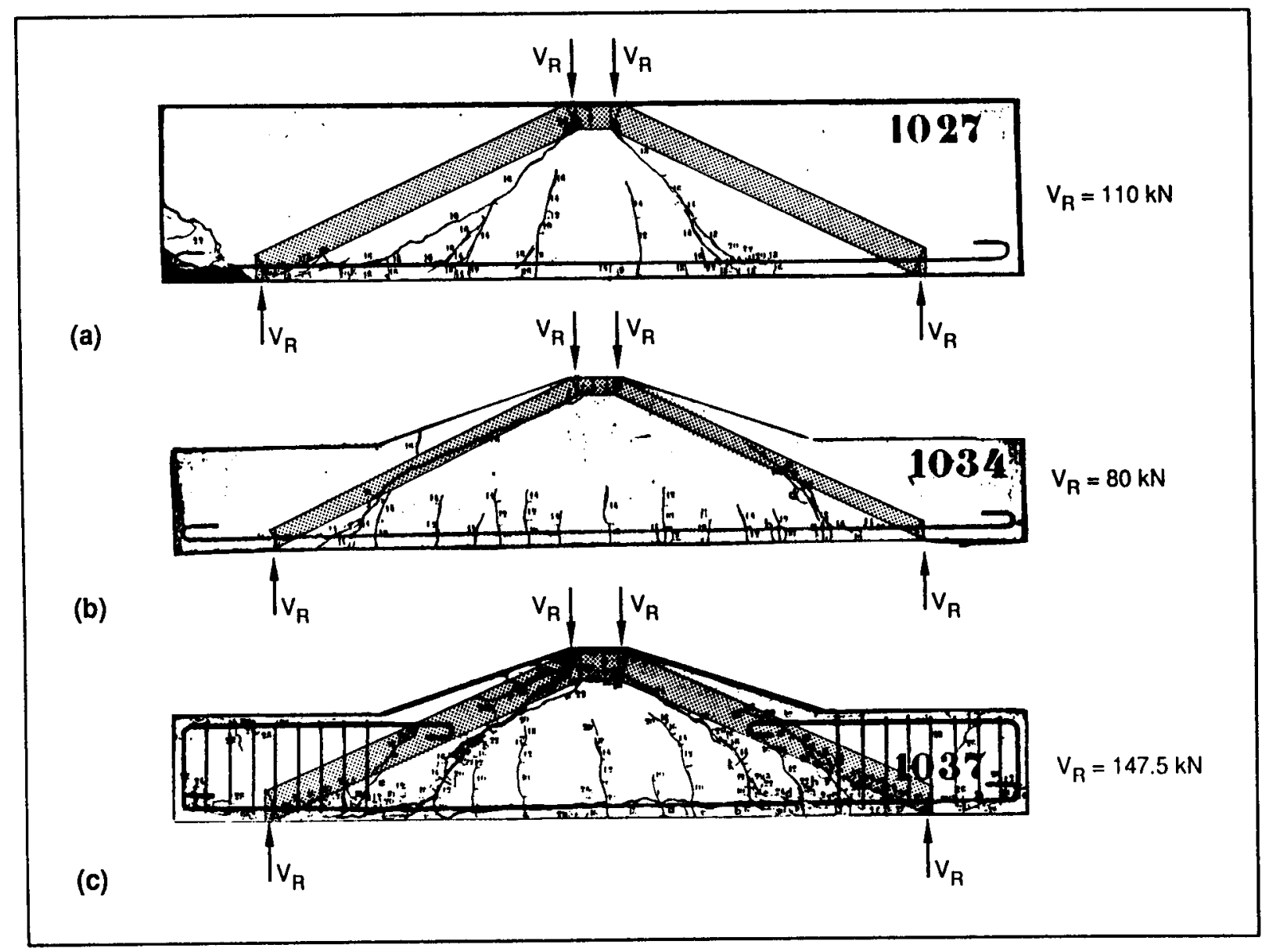

Bild 6.9: Einfluss der Balkengeometrie und der Bewehrung auf das Rissbild und auf die Bruchlast (von Mörsch beschriebene Versuche, 1929)

(a)

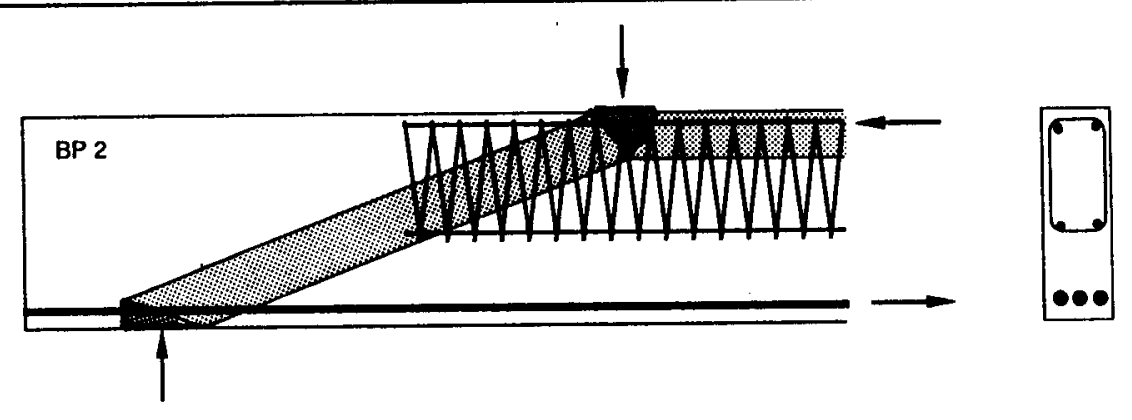

(b)

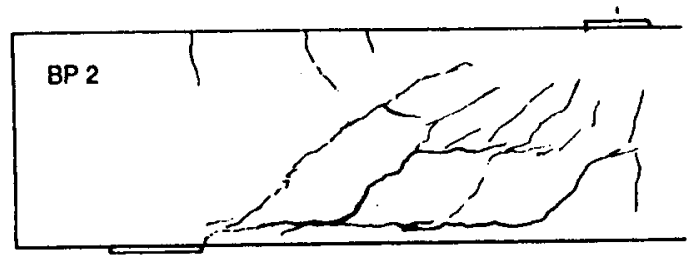

Bild 6.10: (a) Umschnürung der Druckstrebe; (b) Rissbild. 


\subsection{Schubbeanspruchte Druckglleder}

Die Entstehung kritischer Schubrisse in Druckgliedern wird wie bei den vorgespannten Trägern mit einer genügend grossen Normalkraft verhindert. Bild 6.11a zeigt das Spannungsfeld eines unbewehrten Druckgliedes (weder Lăngs- noch Bügelbewehrung).

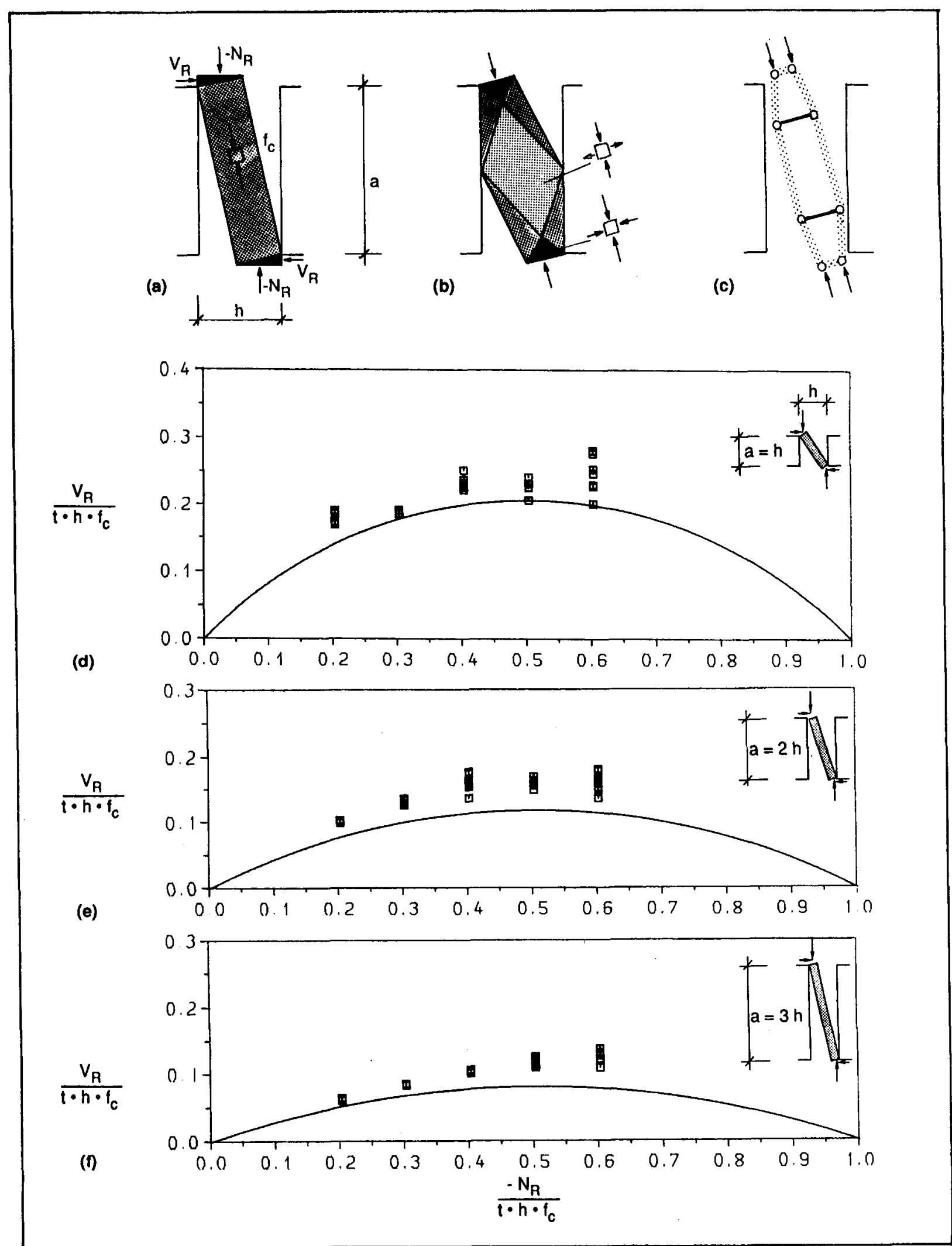

Bild 6.11: Direkte Abstützung: (a) ohne Kraftausbreitung; (b) und (c) mit Kraftausbreitung; (d) bis (f) theoretische Traglast (Direktabstützung ohne Kraftausbreitung) im Vergleich mit Versuchen. 
In diesem Fall kann sich problemlos eine direkte Druckstrebe einstellen. Dieses Tragverhalten wird durch die Bilder 6.11d, e und $f$ bestătigt, in denen die von Tomii, Sakino, Kiyohara (1981) experimentell ermittelte Festigkeit mit der Traglast nach der Plastizitătstheorie verglichen wird (s. Anhang A5). Bei sămtlichen Experimenten ist die theoretische Traglast sogar überschritten worden. Dies kann mit dem im Bild 6.11b dargesteltten Spannungsfeld erklärt werden (Bild 6.11c zeigt die zugehörige Resultierende). Die aus der Kraftausbreitung resultierende Querdruckspannung im Einspannbereich ermöglicht eine Erhöhung der Druckfestigkeit infolge dreiachsiger Wirkung. Die Querzugspannung im mittleren Bereich scheint nicht massgebend zu sein.

\subsection{Durchstanzen}

Randgestützte Platten weisen in der Regel eine derart grosse Schlankheit auf, dass beim Bruch die Lảngsbewehrung fliesst, bevor sich die Biegerisse im Schubbereich fortpflanzen (Bereich $\alpha>\alpha_{3}$ im Bild 6.6). Wenn jedoch eine Platte direkt auf Stützen gelagert ist, kann sich ein Schubbruch einstellen. Im folgenden wird der im Bild 6.12 dargestellte Plattenausschnitt behandelt.

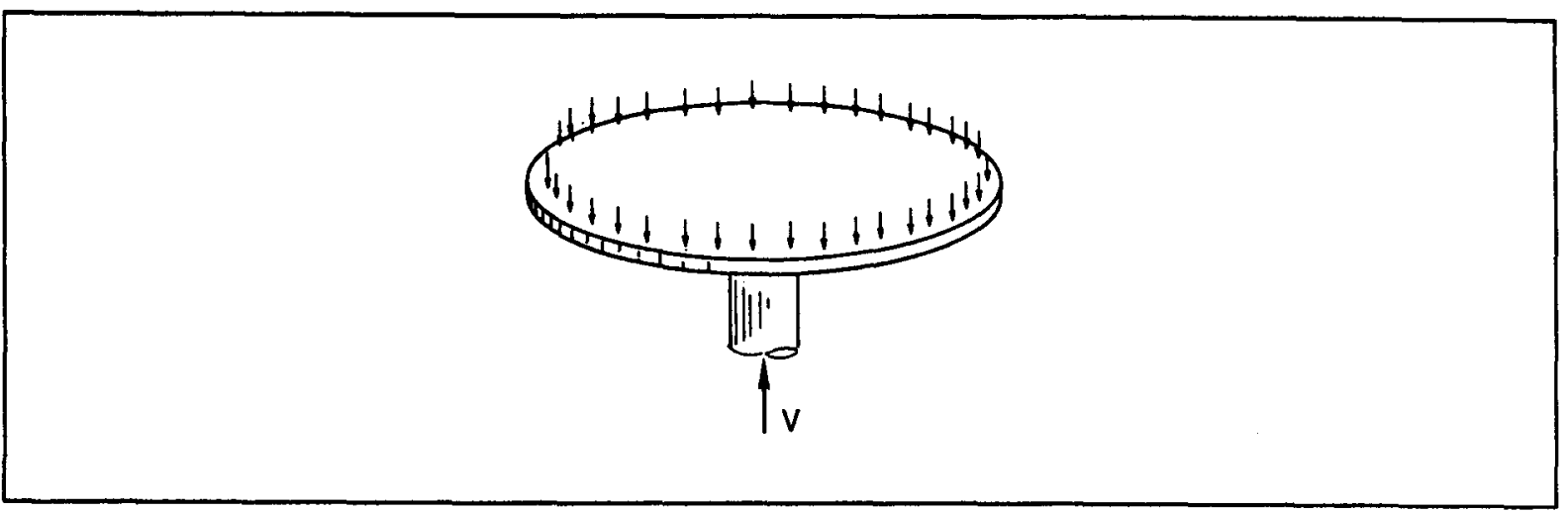

Bild 6.12: Plattenausschnitt im Stützenbereich

Mit kleinen Anpassungen können die Spannungsfelder zur Beschreibung der inneren Tragwirkungen im Balken auch für einen Plattenausschnitt übernommen werden. Bild 6.13a zeigt die Lage der Resultierenden der inneren Kräfte, nachdem sich bereits tangentiale Risse ausgebildet haben.

Wie zahlreiche Durchstanzversuche zeigten, konzentrieren sich die Krümmungen in radialer Richtung im Stützenbereich, so dass sich ringförmige Biegerisse nur in diesem Bereich bilden. In den übrigen Zonen jedoch sind nur gerade radiale Risse vorhanden. Da über radiale Schnitte keine Querkraftübertragung erfolgt, wird der Spannungszustand durch diese Risse nicht gestört. Im tangential gerissenen Bereich kann ein Teil der Querkraft auch über die Verzahnung der Rissufer und durch die Dübelwirkung der Bewehrungsstăbe (siehe Bild 6.4) aufgenommen werden. Aus Bild 6.13a geht hervor, dass die horizontalen Komponenten der Druck- und Zugdiagonalen von der Bewehrung in radialer Richtung, von der radialen Druckzone und auch durch Umlenkung der Bewehrungs- und Druckzonenkraft in tangentialer Richtung aufgenommen werden können. 


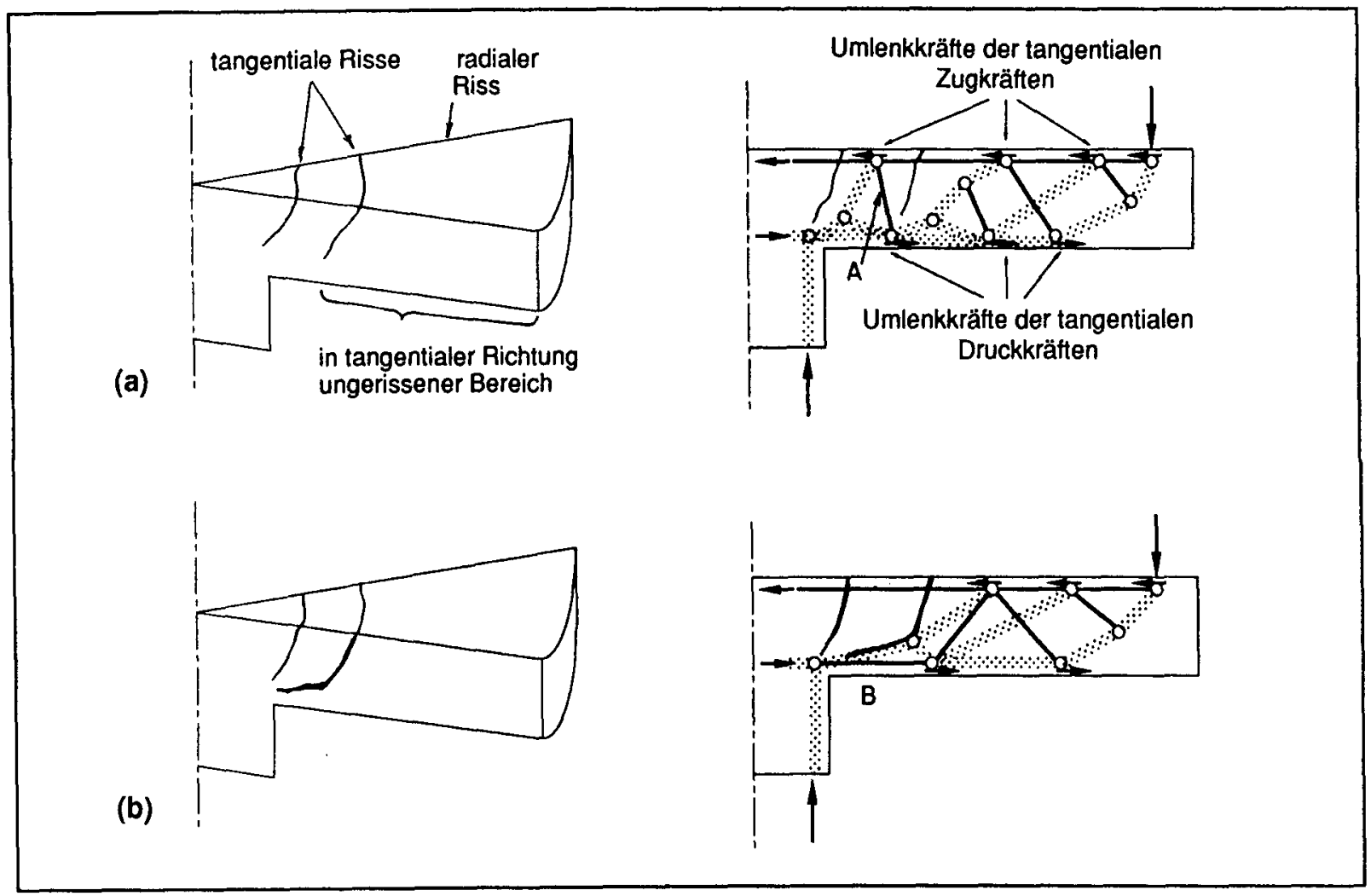

Bild 6.13: Resultierende der inneren Krăfte vor der Fortpflanzung der tangentialen Biegerisse (a) und darnach (b).

Bei einer Erhöhung der Last wird die Festigkeit des Betonzugbandes bei A (Bild 6.13a) erreicht, so dass sich der Biegeriss in horizontaler Richtung fortpflanzt. Die sich damit einstellende neue Tragwirkung gleicht derjenigen des Balkens. Da über den Riss keine Kraftübertragung erfolgt, bildet sich eine geknickte Druckstrebe mit einem ebenfalls geknickten Zugband aus (Bild 6.13b).

Oft wurde experimentell festgestellt, dass die Betonstauchung in radialer Richtung im Druckzonenbereich in der Năhe der Stütze (Punkt B im Bild 6.13b) bei monoton wachsender Last anfänglich zunimmt und nach Erreichen einer gewissen Last wiederum abnimmt, bis sie kurz vor dem Bruch zum Teil negative Werte annimmt (Bild 6.14). Diese Beobachtung steht mit dem vorgeschlagenen Tragmodell im Einklang. Die Stauchung nimmt ab, nachdem sich der Hauptbiegeriss fortgepflanzt hat, und inr negativer Wert (Dehnung) weist darauf hin, dass sich bei B (Bild 6.13b) ein Zugband ausgebildet hat. 


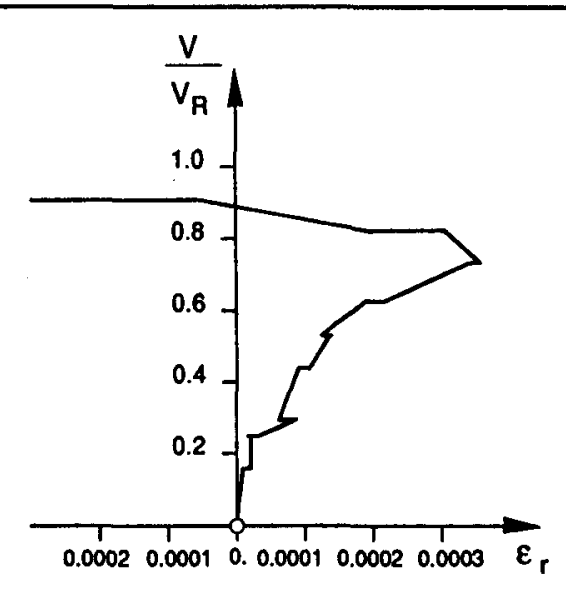

Bild 6.14: Last als Funktion der radialen Betonstauchung in der Druckzone (Versuch IB 15a-14 von Kinnunen, Nylander (1960))

Da die Tragwirkung und die Bruchmechanismen analog denjenigen des Balkens sind, kommen auch bei den Platten die gleichen konstruktiven Lơsungsmöglichkeiten in Frage.

Das Rissbild kann günstig beeinflusst werden, indem im Stützenbereich keine Bewehrung angeordnet wird. Aehnlich wie beim Balken mit Bewehrung ohne Verbund, wo sich die Risse nur in der Năhe der Lasteinleitung ausbilden kőnnen, entstehen bei einer solchen Platte tangentiale Risse nur oberhalb des Stützenrandes. Ein solches Verhalten zeigen die von Bollinger (1985) durchgeführten Versuche an Plattenausschnitten.

Die im Bild 6.15 dargestellten Versuche II, 9a und 11 waren mit konzentrischen Ringen bewehrt, die nur am Plattenrand angeordnet waren. Die Durchstanzlast dieser Versuche war systematisch höher als diejenige ähnlicher Versuche, die auch im Stützenbereich eine Bewehnung hatten. Bei den Versuchen 10a und 12 verursachte sogar die Erhöhung des Bewehrungsgehaltes durch einen zusătzlichen Bewehrungsring im kritischen Bereich eine betrăchtliche Abminderung der Durchstanzlast.

Analog zum Balken (Bild 6.10) kann die Druckstrebe mit einer Umschnürbewehrung verstärkt werden. Bild 6.16a zeigt einen Vorschlag für eine solche konstruktive Lơsung. Wenn diese Umschnürungsbewehrung die Druckzone und die Biegebewehrung (Bild 6.16b) umfasst, kann die Last wie in den Bildern 6.16c und $d$ dargestellt indirekt abgetragen werden. Bild 6.16e zeigt das Rissbild der Schnittfläche einer derartigen Platte, die von Müller, Muttoni, Thürlimann (1984) geprüft worden ist. Die Effizienz dieser Bewehnungsart ist aus der im Bild $6.16 f$ dargestellten Last-VerformungsKurve ersichtlich. Als Verformung ist die Zunahme der Plattendicke dargestellt. Die Phase der Rissfortpflanzung lässt sich daraus erkennen. 


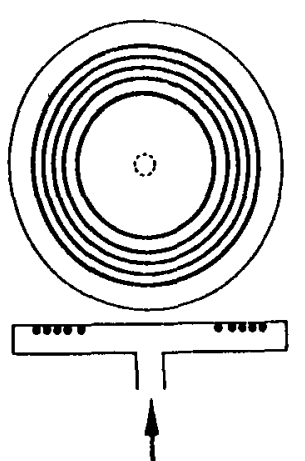

Versuch II

5 Bewehrungsringe $\varnothing 6$ Traglast $=62.5 \mathrm{kN}$

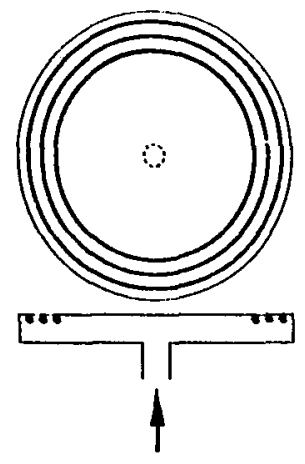

Versuch $9 a$

3 Bewehrungsringe $\varnothing 8$

Traglast $=56.7 \mathrm{kN}$

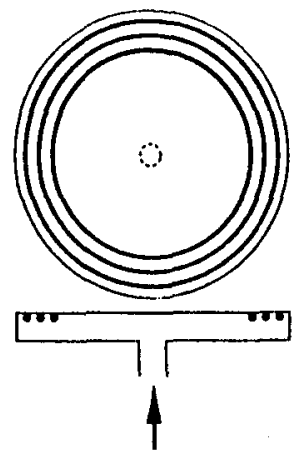

Versuch 11

3 Bewehrungsringe 010

Traglast $=76.1 \mathrm{kN}$

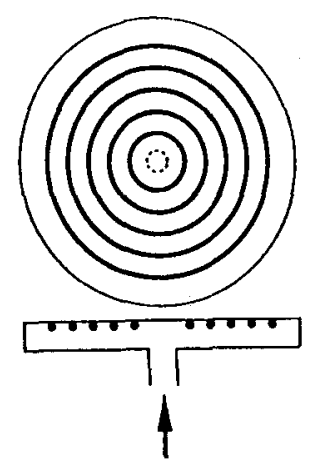

Versuch 1

5 Bewehrungsringe 66

Traglast $=34.2 \mathrm{kN}$

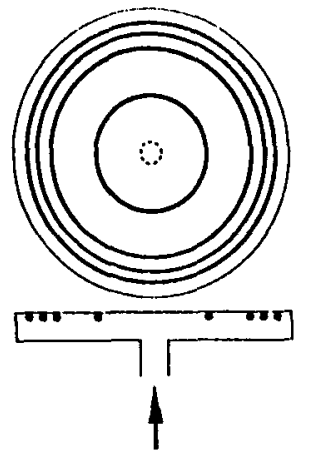

Versuch $10 \mathrm{a}$

3 ø $8+1$ ø 12

Traglast $=41.5 \mathrm{kN}$

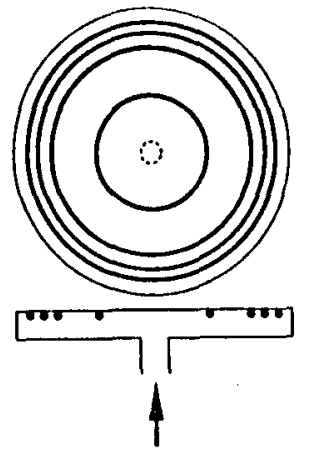

Versuch 12

3 ø $10+1$ ø 12

Traglast $=44.0 \mathrm{kN}$
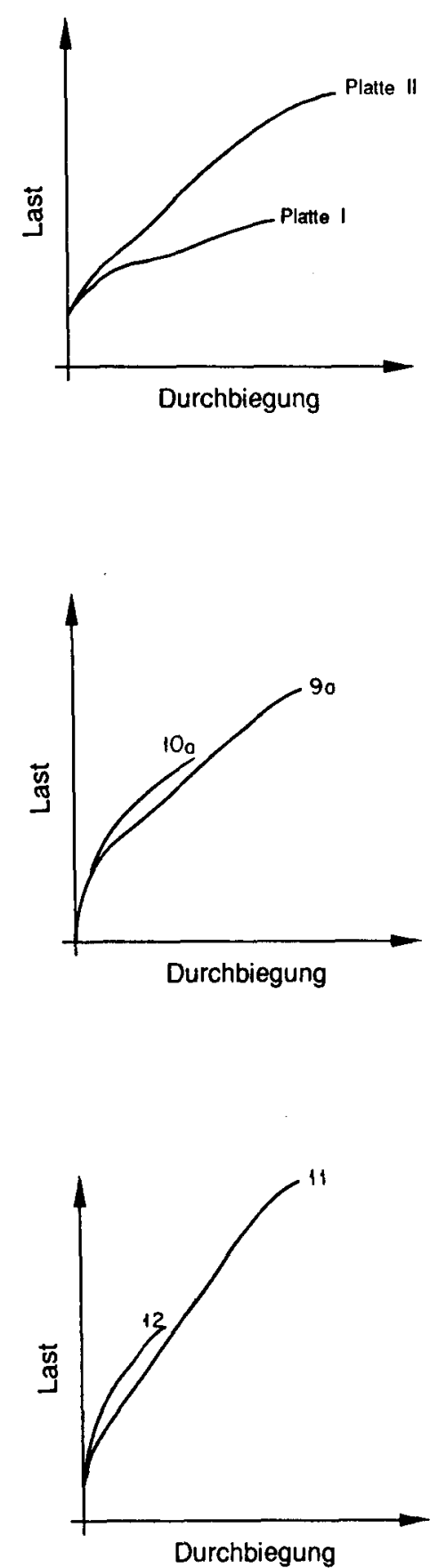

Bild 6.15: Einfluss der Bewehrungsverteilung auf die Bruchlast. Versuche von Bollinger (1985) 
(a)
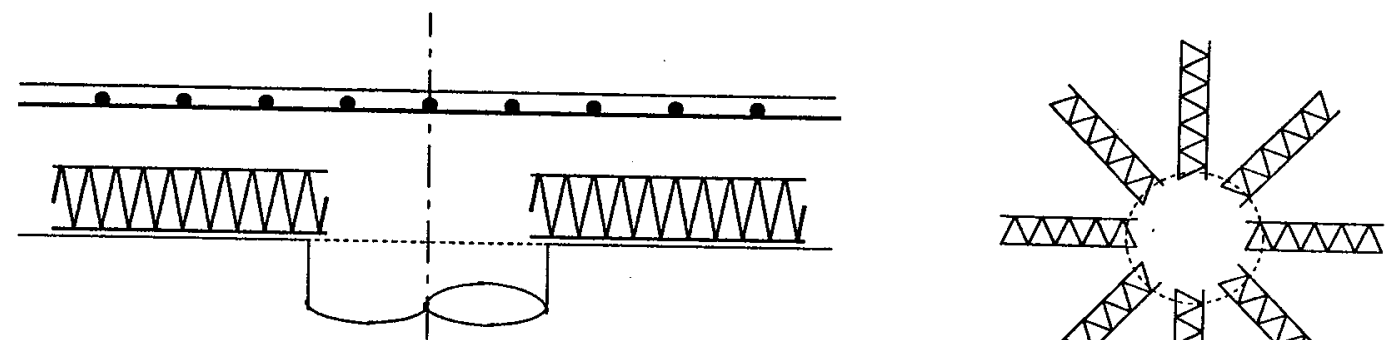

(b)
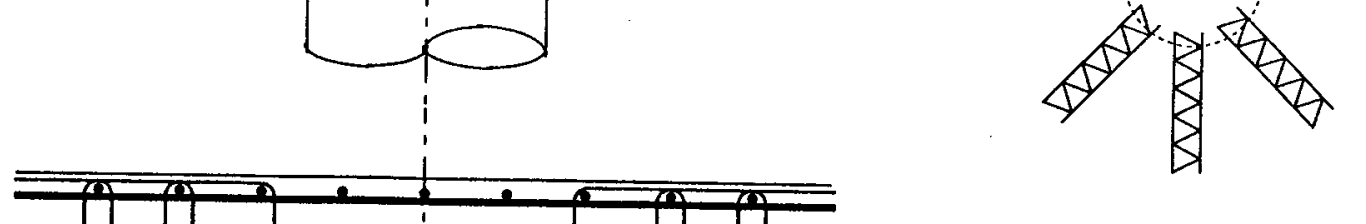

(c)
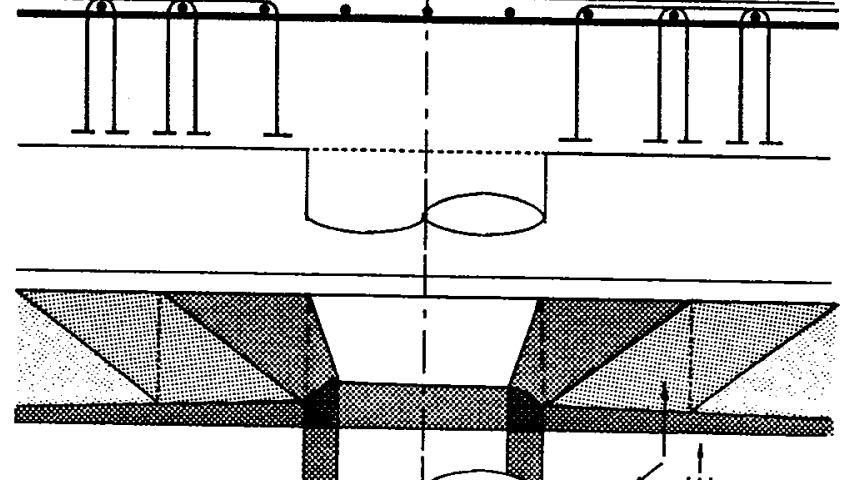

西

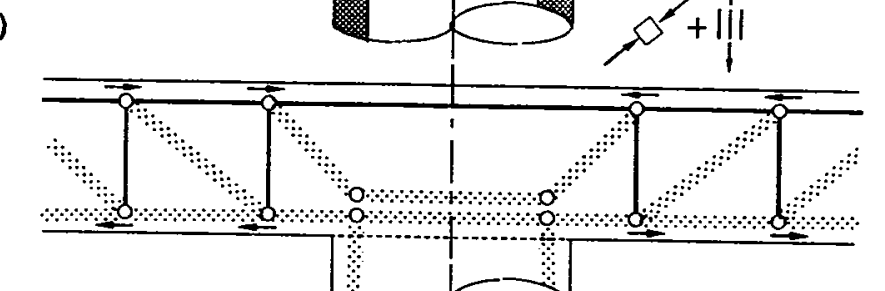

(d)

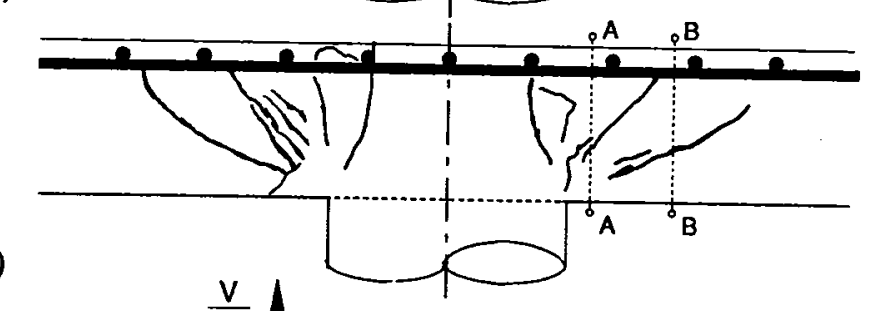

(e)

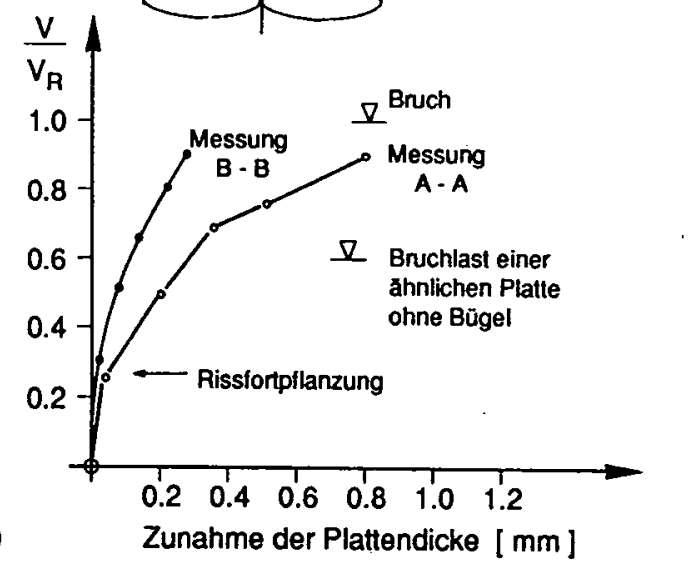

Bild 6.16: Mögliche Plattenverstärkungen: (a) Umschnürungsbewehrung; (b) Bügelbewehrung mit (c) Spannungsfeld und (d) Resultierenden; (e) Rissbild und (f) gemessene Zunahme der Plattendicke 


\section{Verhalten plastisch bemessener Tragwerke im Gebrauchszustand}

Nachdem die Anwendbarkeit der Plastizitătstheorie beim Nachweis der Tragfăhigkeit untersucht ist, wird in diesem Kapitel die Frage beantwortet, ob sich plastisch bemessene Tragwerke auch im Gebrauchszustand befriedigend verhalten. Als Einfühnung wird zuerst das Tragverhalten im Gebrauchszustand eines statisch unbestimmten Tragwerks untersucht.

\subsection{Verhalten eines statisch unbestimmten Tragwerks}

Als repräsentatives Beispiel wird der im Bild 7.1 dargestellte Balken untersucht. Der Träger ist beidseitig eingespannt, symmetrisch belastet und horizontal nur einseitig gehalten, so dass das System einfach statisch unbestimmt ist.

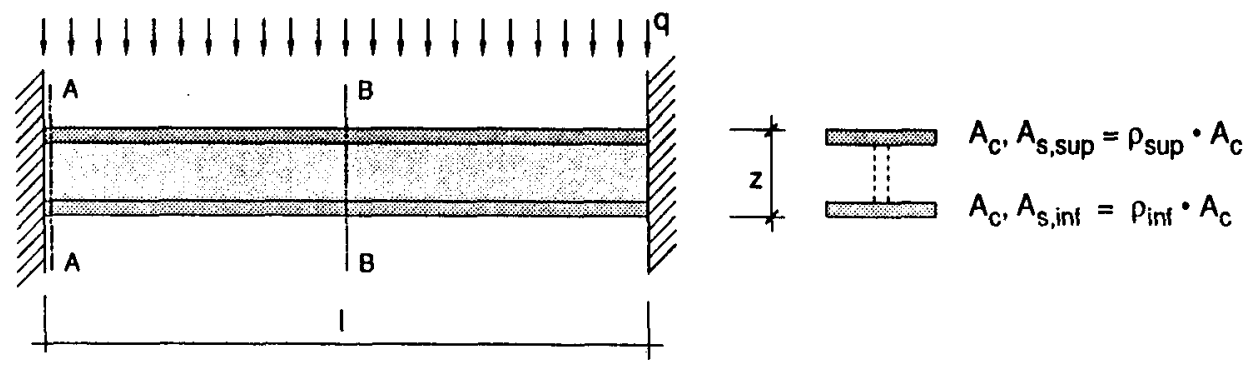

Bild 7.1: Einfach statisch unbestimmtes System

Bild 7.2a zeigt die Momenten-Krümmungs-Beziehung des gewăhlten Querschnittes. Zur Vereinfachung wird ein konstanter Hebelarm $z$ angenommen. Aus dem dargestellten Verlauf sind die bereits im Abschnitt 4.1 enwăhnten Bereiche erkennbar:

- Ungerissener Bereich:

Das Verhalten ist praktisch linear elastisch.

- Gerissener Bereich:

Zugkräfte werden über die Risse nur von der Bewehrung aufgenommen. Der zwischen den Rissen vom Beton aufgenommene Anteil Zugkräfte (tension stiffening) nimmt mit der Belastung ab, weil sich der Verbund zwischen dem Bewehrungsstab und dem Beton löst. Dieses Verhalten ist von zahlreichen Parametern wie Betonzugfestigkeit, Bewehrungsgehalt, Verbundgüte sowie Belastungsart abhängig und kann nur empirisch ermittelt werden. Für die quantitative Untersuchung wird mit den im Bild 4.1 dargestelten Beziehungen gerechnet. Die Vernachlässigung der Betonzugkräfte würde zu einem linearen Verlauf der Momenten-Krümmungs-Beziehung führen (gestrichelte Linie im Bild 7.2a).

- Plastischer Bereich:

Die Bewehrung fliesst, und wenn der Hebelarm nicht abnimmt (keine Entfestigung des Druckgurtes), erfolgt bei Zunahme der Krümmung keine Aenderung der Beanspruchung. 
(a)
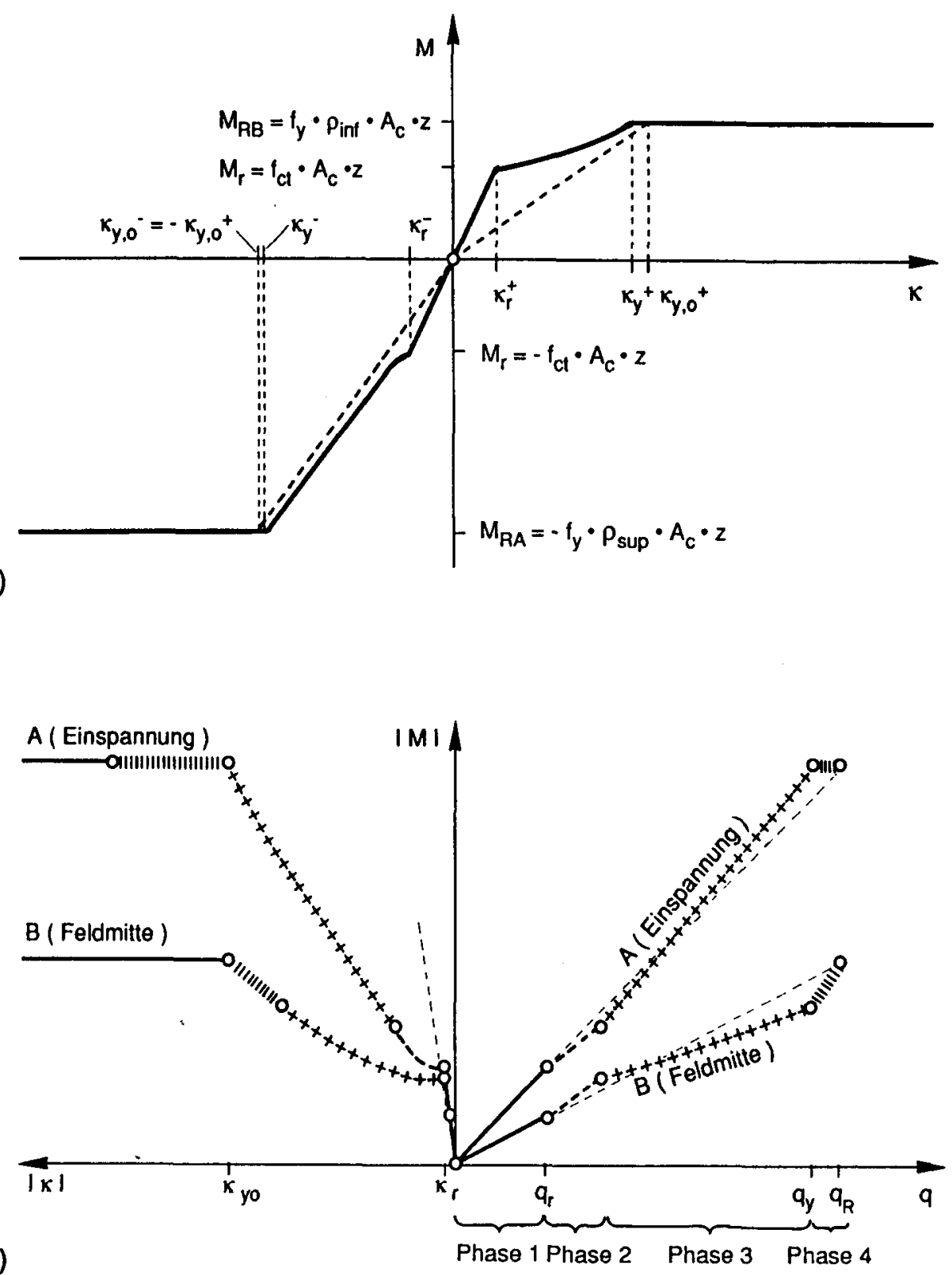

Bild 7.2: (a) Momenten-Krümmungs-Beziehung des gegebenen Querschnittes mit üblichen Bewehrungsgehalten und (b) Biegemomente als Funktion der Last.

Da hier vor allem das Tragverhalten im Gebrauchszustand behandelt wird, wird auf eine Untersuchung des Einflusses der Betonentfestigung und der Stahlverfestigung verzichtet. Die drei beschriebenen Bereiche lassen sich auch im Tragverhalten des Systems erkennen. Bild 7.2b zeigt die Momente im Schnitt A (Einspannmoment) und im Schnitt B (Feldmoment) in Funktion der Last q (Eigengewicht plus Nutzlasten).Bei monotoner Erhöhung der Belastung werden folgende Phasen durchlaufen: 
- Phase 1:

Der Trăger ist überall ungerissen. Bei konstanter Biegesteifigkeit ist bekanntlich das Einspannmoment im Fall eines eigenspannungslosen Zustandes doppelt so gross wie das Feldmoment: $\left|M_{A}\right|=2 \cdot M_{B}$

- Phase 2:

Nach der Ueberschreitung der Risskrümmung $x_{r}$ sind bei der Einspannung die ersten Risse vorhanden, im Feldbereich hingegen ist der Träger noch ungerissen. Die Beziehung $\left|M_{A}\right|=2 \cdot M_{B}$ ist nicht mehr gültig.

- Phase 3:

Der Trăger ist auch im Feldbereich gerissen.

- Phase 4:

Im Normalfall (Ausnahmen werden später behandelt) bleibt auch in der gerissenen Phase die Krümmung bei der Einspannung grösser als diejenige im Feld, so dass üblicherweise die Bewehrung zuerst im Einspannbereich fliesst. Die Bildung eines solchen plastischen Gelenkes ist mit der Bildung grosser Risse verbunden. Da die Bewehrung im Feldbereich noch nicht geflossen ist, kann in dieser Phase die Last noch weiter erhőht werden.

- Phase 5:

Die Traglast wird erst beim Fliessen der Bewehrung auch im Feldbereich erreicht. Mit der angenommenen Momenten-Krümmungs-Beziehung bleibt bei weiterer Vertormungszunahme die Last konstant.

\subsection{Elastische und plastische Bemessungsmethoden}

Unter der Voraussetzung, dass die Tragtăhigkeit massgebend ist, wird die erforderliche Bewehrung von Stabtragwerken so bemessen, dass die Beanspruchungen (Schnittkrăfte) im Gebrauchs- oder im Bruchzustand nicht grősser sind als die zulässigen Beanspruchungen resp. die Querschnittswiderstände. Die folgende Tabelle zeigt die üblichen Methoden.

\begin{tabular}{|c|c|c|}
\hline Methode & Ermittlung der Schnittkräfte & Berechnung des Schnittkräftewiderstandes \\
\hline 1 & elastisch & elastisch \\
2 & elastisch & plastisch \\
3 & plastisch & plastisch \\
\hline
\end{tabular}


Bei der Ermittlung der Schnittkraftverteilung aufgrund der Elastizitătstheorie wird üblicherweise das Momenten-Krümmungs-Verhalten gemăss Bild 7.2a vereinfacht; das Verhalten wird linearisiert

$M=E I \cdot \kappa$

Damit die Abhängigkeit der Biegesteifigkeit vom noch unbekannten Bewehrungsgehalt vernachlässigt werden kann, wird im allgemeinen die Steifigkeit El im ungerissenen Bereich berücksichtigt. Die Folgen dieser Annahmen werden spăter aufgezeigt.

Die massgebenden Momente für die Bemessung des beidseitig eingespannten Balkens (Bild 7.1) sind:

$\begin{array}{ll}\text { - } & \text { Obere Bewehrung: } \quad M_{A}=-2 \cdot M_{B}=-\frac{q \cdot l^{2}}{12} \\ \text { - Untere Bewehrung: } & M_{B}=\frac{q \cdot l^{2}}{24}\end{array}$

Die plastische Ermittlung der Schnittkrăfte basiert dagegen auf dem statischen Grenzwertsatz der Plastizitătstheorie. Dieser besagt, dass eine Belastung, die zu einem Spannungsfeld gehört, das die Gleichgewichtsbedingungen und die statischen Randbedingungen erfült und die Fliessbedingung nicht verletzt, ein unterer Grenzwert der Traglast ist. Für die Bemessung kann dieser Grenzwertsatz wie folgt umformuliert werden:

"Bei der plastischen Bemessung ist ein Spannungsfeld (Schnittkraftverteilung) zu wählen, das die Gleichgewichtsbedingungen sowie die statischen Randbedingungen erfüllt. Die Querschnittabmessungen und die Bewehrung sind so $\mathrm{zu}$ bemessen, dass die Widerstände überall grösser sind als die Beanspruchungen."

Für diesen Balken führen die Gleichbewichtsbedingungen zum folgenden Ausdruck:

$$
\left|M_{A}\right|+M_{B}=\frac{q \cdot l^{2}}{8}
$$

Nach der Plastizitătstheorie sind somit sămtliche Momentendiagramme zulässig zwischen den Extremen

$$
\left|M_{A}\right|=0, \quad \quad M_{B}=\frac{9 \cdot \ell^{2}}{8} \quad \text { (untere Momentenlinie im Bild 7.3) }
$$

und

$$
\left|M_{A}\right|=\frac{g \cdot l^{2}}{8}, \quad M_{B}=0 \quad \text { (obere Momentenlinie) }
$$

Wird jedoch der Stahlbetonbalken aufgrund einer dieser beiden Extreme bemessen, so wird er die üblichen Kriterien der Gebrauchstauglichkeit nicht erfüllen. Im Abschnitt 7.3 wird erläutert, nach welchen Kriterien die Wahl einer statisch zulăssigen Momentenlinie getroffen werden muss, damit auch die Gebrauchstauglichkeit eingehatten wird. 


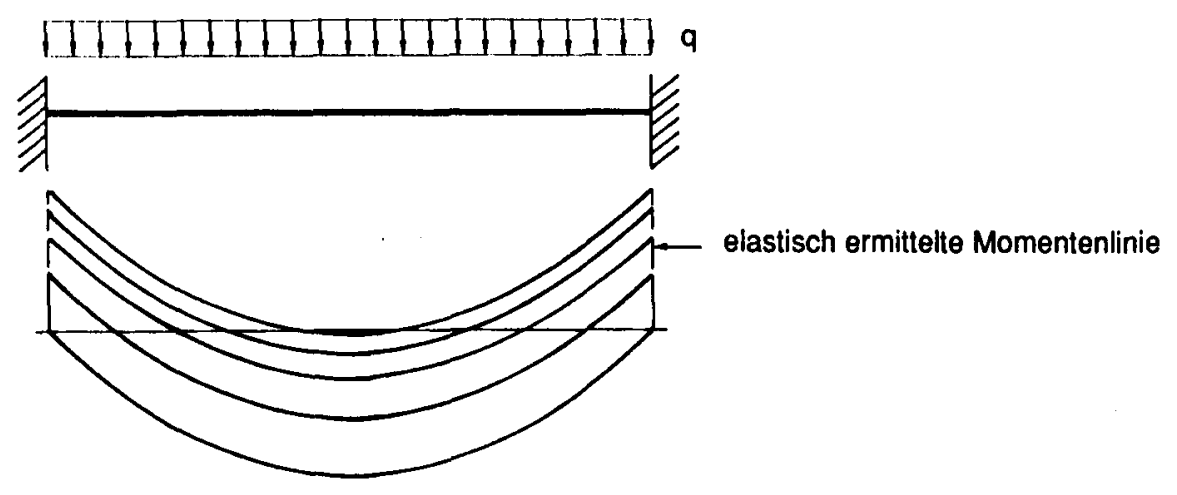

Bild 7.3: Statisch zulässige Momentenlinien

Die elastisch oder plastisch ermittelten Beanspruchungen müssen entweder mit den zulässigen Beanspruchungen resp. mit dem Widerstand verglichen werden. Die zulässige Beanspruchung ist diejenige Schnittkraft, bei der die zulăssige Spannung entweder im Stahl oder im Beton erreicht wird.

Für die Berechnung der zulăssigen Spannung werden im allgemeinen folgende Annahmen und Vereinfachungen getroffen:

- Die Stoffgesetze von Stahl und Beton sind linear elastisch.

- Die Dehnungen in Längsrichtung sind über die Balkenhöhe linear verteilt.

- Der Beton nimmt keine Zugkraft auf (kein tension stiffening).

Aus diesen Annahmen geht hervor, dass die so ermittelten Spannungen mit den effektiv vorhandenen Spannungen nicht übereinstimmen und als nominelle Grössen zu betrachten sind.

Die erforderliche Bewehrungsmenge wird so bestimmt, dass die zulässige Beanspruchung gleich der Beanspruchung im Gebrauchszustand ist. Bei nicht zu stark bewehrten Querschnitten ist die Stahlspannung massgebend

$$
M_{z u l}=A_{s} \cdot \sigma_{z u l} \cdot z
$$

so dass die erforderliche Bewehrungsfläche

$$
A_{S}=\frac{M}{\sigma_{z u I^{-Z}}}
$$

ist. Da im vorliegenden Fall der Hebelarm nur wenig variiert, resultiert für den oberen Flansch ein Bewehrungsgehalt, der doppelt so gross ist wie derjenige des unteren Flansches, weil das elastisch ermittelte Moment im Einspannbereich ebenfalls doppelt so gross ist als dasjenige in Feldmitte. 
Es ist zu bemerken, dass die Annahmen, die bei der elastischen Ermittlung der Momente und bei der Berechnung der Spannungen getroffen wurden, nicht kompatibel sind. Bei der Berechnung der Schnittkräfte wurde eine bewehrungs- und schnittkraftunabhăngige konstante Steifigkeit angenommen, bei der Berechnung der Spannungen hingegen wurde die Betonzugfestigkeit vemachlässigt, so dass die Biegesteifigkeit direkt proportional zum Bewehrungsgehalt ist. Deshalb sind auch die Resultate nicht kompatibel. Das Moment und somit auch die Krümmung sind im Einspannbereich doppelt so gross wie in Feldmitte. Die Bewehrung hingegen wurde so bestimmt, dass die Stahlspannung in diesen beiden massgebenden Schnitten gleich der zulässigen Spannung ist; somit sind die Dehnung und die Krümmung auch gleich. Diese Unvertrăglichkeit kann beseitigt werden, indem die Abhăngigkeit der Steifigkeit vom Bewehrungsgehalt und von der Beanspruchung berücksichtigt wird. Eine solche Bemessungsmethode ist jedoch zu autwendig, weil ein iteratives Verfahren durchzuführen wăre. Die Folge dieser Unverträglichkeit besteht darin, dass im am meisten beanspruchten Querschnitt die Bewehrung im allgemeinen fliesst, bevor die Traglast erreicht wird. Diese wird erst nach der Umlagerung der inneren Krätte infolge plastischer Verformungen erreicht, obwohl der Trăger elastisch berechnet worden ist.

Die Ermittlung des Biegewiderstandes enfolgt im allgemeinen aufgrund der Plastizitătstheorie. Die Lösung aufgrund der statischen Methode ist im Abschnitt 4.2 beschrieben (Bild $4.9 \mathrm{~b}$ ). In diesem Fall betrăgt der Biegewiderstand

$$
M_{R}=A_{s} \cdot f_{y} \cdot z
$$

mit dem geschắtzten Hebelarm z. Die erforderliche Bewehnungsflăche betrăgt somit

$$
A_{s}=\frac{M_{R}}{f_{y} \cdot z}=\frac{\gamma \cdot z}{y^{\cdot z}}
$$

wobei $\gamma$ der Sicherheitsfaktor ist.

Da die zulăssige Spannung $\sigma_{z u l}$ und die mit dem Sicherheitsfaktor $\gamma$ reduzierte Streckgrenze $f_{y} / \gamma$ etwa gleich gross sind, resultiert aufgrund der Bemessungsmethode mit elastischer Schnittkraftermittlung und Bestimmung des Widerstandes nach Plastizitătstheorie etwa die gleiche Bewehrung wie mit der Bemessung aufgrund der Methode der zulässigen Spannungen (Ermittlung der Schnittkräfte und Querschnittsbetrachtung aufgrund der Elastizitătstheorie). In diesem Fall erhălt man auch mit dieser Bemessungsmethode eine Bewehrung, die im oberen Flansch doppelt so stark ist wie im unteren

$$
\frac{\rho_{\text {sup }}}{\rho_{\text {inf }}}=2
$$

Wenn der Trăger plastisch bemessen wird, kann das Verhăltnis $\rho_{\text {sup }} / \rho_{\text {inf }}$ frei gewăhlt werden.

Der Einfluss des Verhăltnisses $\rho_{\text {sup }} / \rho_{\text {inf }}$ auf das Tragverhalten wird im folgenden Abschnitt behandelt. 


\subsection{Elnfluss der Bemessungmethode auf das Verhalten Im Gebrauchszustand}

Die Gebrauchstauglichkeit von Stahlbetontragwerken wird durch zu breite Risse, zu grosse Durchbiegungen sowie Schwingungen mit zu grosser Amplitude beeintrăchtigt. Im allgemeinen können zu grosse Durchbiegungen und Schwingungsamplituden mit der Wahl einer angepassten Trägerschlankheit und/oder mit einer geeigneten Vorspannung vermieden werden. $\mathrm{Zu}$ breite Risse entstehen infolge schlechter Aufteilung der Bewehrung, ungenügender Verbundeigenschaften oder, weil die Bewehrung bereits im Gebrauchszustand geflossen ist. Wie bereits gezeigt (Bild 7.2b) tritt bei statisch unbestimmten Systemen die erste Plastifizierung der Bewehrung bei einer Last $q_{y}$ ein, die im allgemeinen kleiner ist als die Traglast $q_{R}$. Dies enfolgt selbst dann, wenn das Tragwerk elastisch bemessen worden ist. Da die plastische Verformung der Bewehrung mit der Bildung breiter Risse verbunden ist, kann das Verhăltnis $q_{y} / q_{R}$ als Mass für die Gebrauchstauglichkeit eines Tragwerks interpretiert werden. Damit sich im Gebrauchszustand nicht zu breite Risse entwickeln, muss das Tragwerk so bemessen werden, dass diese Grösse den Wert $1 / \gamma$ übersteigt.

Wie bereits enwăhnt, müssen bei der Ermittlung der Fliesslast $q_{y}$ sowohl der Eigenspannungszustand als auch der genaue Verlauf des Momenten-Krümmungs-Diagramms bekannt sein. Als Eigenspannungszustand wurde bei der Behandlung des im Bild 7.1 dargestelten Tragwerks stellvertretend eine Temperaturdifferenz zwischen dem Ober- und dem Untergurt berücksichtigt. Als Stoffgesetz wurde der vom CEB-FIP Code Modèle (1978) vorgeschlagene empirische Verlauf (Bild 4.1b) angenommen. Die Ermittlung der Schnittkrăfte erfolgte wegen der Nichtlinearităt des Stoffgesetzes iterativ. Das Einspannmoment wurde variiert, bis das Integral der Krümmung über die Balkenlănge gleich null war (100 Integrationspunkte pro Balkenhälfte). Bild 7.4 zeigt die Resultate. Das Verhältnis $q_{y} / q_{R}$ ist als Funktion des Bewehrungsverhältnisses $\rho_{\text {sup }} / \rho_{\text {inf }}\left(\rho_{\text {sup }}+\rho_{\text {inf }}=\right.$ konstant) sowie der Zwängung (Temperaturdifferenz) dargestellt. Der Vergleich der beiden Diagramme mit verschiedenen Zugfestigkeiten ( $f_{c t}=2 \mathrm{MPa}$ im Bild 7.4a und $f_{c t}=3 \mathrm{MPa}$ im Bild 7.4b) bestätigt, dass die Fliesslast von den verschiedenen Parametern stark abhăngig ist. Auch die Tatsache, dass bei einem elastisch bemessenen statisch unbestimmten Tragwerk im allgemeinen eine Umlagerung der inneren Kräfte nach der ersten Plastifizierung erfolgt, wird mit diesen Diagrammen bestätigt; mit einem Verhăltnis obere/untere Bewehrung $\rho_{\text {sup }} / \rho_{\text {inf }}=2$ ist das Verhältnis Fliesslast $/ T$ raglast $q_{y} / q_{R}$ kleiner als eins.

Wenn der untere Flansch stärker bewehrt wird als der obere $\left(\rho_{\text {sup }} / \rho_{\text {inf }}<1\right)$, resultiert für das Verhältnis $q_{y} / q_{R}$ ein zu kleiner Wert. Bei einer plastischen Bemessung ist somit eine solche Wahl $\mathrm{zu}$ vermeiden. Der obere Flansch wird im Einspannbereich im allgemeinen stärker gedehnt als der untere Flansch im Feldbereich, so dass die erste Plastifizierung im Einspannbereich erfolgt. Je stărker die Bewehrung im oberen Flansch im Verhăltnis zu derjenigen im unteren Flansch ist, desto höher ist somit die Fliesslast. Dieser Sachverhalt lässt sich mit der folgenden Regel verallgemeinern:

"Bei statisch unbestimmten Tragwerken sind vor allem diejenigen Elemente stärker zu bewehren, bei denen die Widerstände zuerst aktiviert werden, d.h. dort, wo die spezifischen Verformungen (Dehnungen, Krümmungen) am grössten sind."

Die Berücksichtigung dieser Regel bei der plastischen Bemessung führt zu einem ausreichend guten Verhalten im Gebrauchszustand. Das Verhalten eines derart plastisch bemessenen Tragwerks kann so- 
gar besser sein als dasjenige eines elastisch bemessenen Tragwerks. Nur bei sehr grossen Verhăltnissen $\rho_{\text {sup }} / \rho_{\text {inf }}$ fliesst die Bewehrung zuerst in Feldmitte und eine weitere Erhöhung dieses Verhăltnisses führt zu einer Reduktion der Fliesslast. In bezug auf die Wahl des Bewehrungsverhăltnisses ist ein grosser Spielraum für die Losungen mit einem guten Tragverhalten im Gebrauchszustand vorhanden.

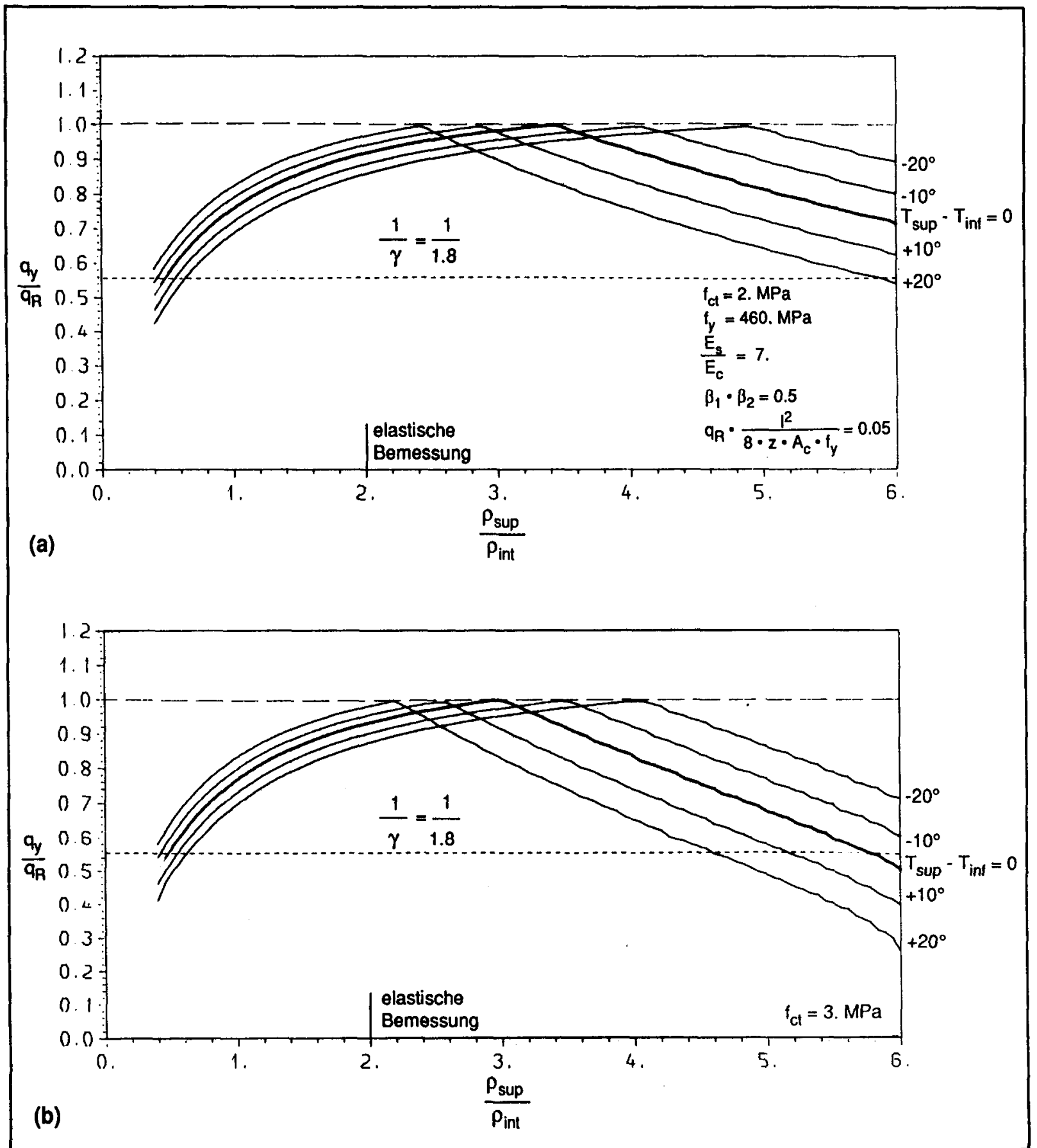

Bild 7.4: Verhăltnis Fliesslast zu Traglast als Funktion des Verhăltnisses $\rho_{\text {sup }} / \rho_{\text {inf }}$ (obere/untere Bewehrung) und der Zwăngung 


\section{Zusammenfassung und Schlussfolgerungen}

In der vorliegenden Arbeit wird untersucht, ob die Plastizitätstheorie in der Bemessung von Stahlund Spannbeton anwendbar ist. Es wird damit versucht, die Frage "Wird bei einem plastisch bemessenen System die Tragsicherheit mit genügender Zuverlăssigkeit geschătzt, und verhălt sich das System auch im Gebrauchszustand befriedigend?" zu beantworten. Die Beantwortung dieser Frage erfordert die getrennte Untersuchung des Tragverhaltens im Bruchzustand und im Gebrauchszustand.

Die Traglast eines statisch unbestimmten Systems kann mit der Plastizitătstheorie überschătzt oder unterschätzt werden, wenn entweder die Annahme eines ideal plastischen Verhaltens der Wirklichkeit nicht entspricht, oder bei der Formulierung der Gleichgewichtsbedingung zu grosse Verformungen nicht berücksichtigt werden (oder, wenn beides zutrifft). In dieser Arbeit werden nur diejenigen Abweichungen von der Plastizitätstheorie betrachtet, die eine Reduktion der Bruchlast verursachen. Es handelt sich dabei um die Entfestigung und die Effekte 2. Ordnung bei Druckgliedern.

Bei der Entfestigung sinkt der Widerstand nach dem Erreichen der Festigkeit mit der Zunahme der Verformung. Die Entfestigung eines Systems erfolgt vor allem rasch, wenn die bis zum Erreichen der Bruchlast gespeicherte Energie (elastische Verformung) im Vergleich zur dissipierten Energie im Bruchvorgang (plastische Verformung) gross ist. Da die gespeicherte Energie eine Funktion der Systemant, dessen Grösse und der Beanspruchungsart ist, beeinflussen nebst den Materialeigenschaften auch diese Faktoren die Sprödigkeit eines Systems. Diese kann mit dem sogenannten Entfestigungsstabilitătsfaktor beschrieben werden, der als Verhältnis der gespeicherten Energie zur dissipierbaren Energie definiert ist. Ausser der Materialentfestigung können auch die Effekte 2. Ordnung die Entfestigung eines Systems verursachen.

Das mechanische Verhalten von Stahl, Beton und Stahlbeton wird mit der Auswertung zahlreicher Versuche beschrieben. Besonders ausführlich wird die Făhigkeit, im Bruchvorgang Energie zu dissipieren, diskutiert. Diese Energiemenge lässt sich durch konstruktive Massnahmen wie Anordnung einer geeigneten Querbewehrung und einer gut gehaltenen Druckbewehrung günstig beeinflussen.

Aus der Untersuchung eines einfach statisch unbestimmten Systems, bestehend aus zwei parallel geschalteten Subsystemen, wird erlăutert, dass die Bruchlast von der Entfestigungscharakteristik des zuerst plastifizierten Subsystems beeinflusst wird. Die Entfestigung des Subsystems, welches zuletzt plastifiziert wird, beeinflusst nach dem Erreichen der Bruchlast das Systemverhalten, nicht aber die Bruchlast selbst. Damit in einem statisch unbestimmten System die Widerstände einzelner Subsysteme im Bruchzustand möglichst ausgenützt werden können, ist das System so zu entwerfen, dass die duktilen Subsysteme möglichst frühzeitig plastifiziert und die spröden Elemente möglichst spät aktiviert werden. Sind mehrere spröde Subsysteme vorhanden, ist eine möglichst gleichzeitige Aktivierung der Widerstände dieser Elemente vorteilhaft.

Als repräsentatives Beispiel von Systemen, bei denen die plastischen Verformungen in Biegegelenken erfolgen, wird ein beidseitig eingespannter Träger untersucht. Die Bruchlast wird beeinflusst vom Eigenspannungszustand (grosse oder kleine Reduktion der Traglast infolge Entfestigung, je nach Eigenspannung), von der Schlankheit des Trăgers (die plastisch dissipierbare Energie ist nur von 
Querschnitt abhăngig, die elastisch gespeicherte Energie nimmt hingegen mit der Lănge des Trăgers zu, so dass die Sprödigkeit des Systems mit der Schlankheit zunimmt) sowie von der Ausbildung des Querschnittes (dissipierbare Energie). Im Fall einer wirksamen Querbewehrung in der Druckzone des Trăgers verhălt sich das System duktil, und die Traglast kann unabhăngig vom Eigenspannungszustand und auch bei grossen Schlankheiten mit guter Genauigkeit mit der Plastizitătstheorie ermittelt werden.

Einige Beispiele von Systemen, bei welchen die plastischen Verformungen in Druckstäben erfolgen, werden behandelt. Da die Vielfalt solcher Systeme gross ist, lassen sich keine allgemein gültigen Schlussfolgerungen ziehen.

Bei Systemen mit plastischen Verformungen in den Schubgelenken mit Betonversagen im Steg ist hingegen die dissipierbare Energie im Vergleich zur Energie, die normalenweise elastisch gespeichert wird, so klein, dass oft nach Erreichen der Schubfestigkeit eine Entfestigung des Systems erfolgt. In diesen Fällen ist die Anwendung der Plastizitătstheorie somit problematisch.

Die Plastizitătstheorie kann jedoch mit Erfolg auch bei Systemen mit sproden Elementen angewendet werden. Diese Elemente können mit der Plastizitătstheorie so bemessen werden, dass sie mit Sicherheit nicht massgebend werden. Eine solche Bemessungsmethode wird in dieser Arbeit vorgeschlagen. Damit lassen sich auch komplexe Systeme sehr einfach bemessen.

Bei Stahlbetonelementen, die eine ungenügende Querbewehrung aufweisen, können im Bruchzustand sehr breite Risse entstehen, über die mangels Verzahnung der Rissufer eine ungenügende Kraftübertragung erfolgt. Einige solche Fälle wie Balken und Druckglieder ohne Schubbewehrung sowie Platten im Stützenbereich werden behandelt. Die Anwendung der Plastizitătstheorie führt in diesen Fällen zu einer Ueberschătzung der Traglast. Ein Vergleich der nach dieser Theorie vorgeschlagenen Tragwirkung mit derjenigen, die sich tatsăchlich einstellt, ermoglicht es, die Bruchursache zu erkennen und geeignete konstruktive Lősungen zu entwickeln.

Die Tragwirkung, die dem plastizitătstheoretischen Modell entspricht, kann sich einstellen, wenn das Rissbild mit einem geeigneten Eigenspannungszustand (z.B. Vorspannung), mit einer sinnvollen Formgebung des Trägers und vor allem mit einer Rissverteilbewehrung günstig beeinflusst wird. Mit diesen Massnahmen kann ausser einem duktileren Tragverhalten auch die plastizitătstheoretische Traglast erreicht werden.

Zuletzt wird die Frage, ob sich plastisch bemessene Tragsysteme auch im Gebrauchszustand befriedigend verhalten, beantwortet. Bei statisch unbestimmten Systemen tritt im allgemeinen die erste Plastifizierung ein, bevor die Traglast erreicht wird. Erfolgt dies bei Lasten, die dem Gebrauchszustand entsprechen, können in diesem Zustand sehr breite Risse entstehen, die die Gebrauchstauglichkeit beeintrăchtigen. Die Bildung plastischer Gelenke und die Umlagerung der inneren Krăfte erfolgen nicht nur bei plastisch bemessenen Systemen, sondern auch bei elastisch bemessenen, weil das wirkliche Last-Verformungs-Verhalten von Stahlbeton im allgemeinen nicht berücksichtigt wird. Plastisch bemessene Systeme verhalten sich je nach Wahl der Widerstănde besser oder schlechter als elastisch bemessene Systeme. Je grösser die Fliesslast (Last beim Erreichen des ersten plastischen Gelenks), desto besser ist das Verhalten im Gebrauchszustand. Dies ist der Fall, wenn die Subsysteme die zuerst geflossen sind, einen grossen Anteil der Traglast aufnehmen. Das bedeutet, dass 
$-124-$

die Tragelemente, die durch grosse spezifische Verformungen gekennzeichnet sind (und somit zuerst fliessen), am stärksten zu bewehren sind. Derart bemessene Systeme enüllen die Gebrauchstauglichkeitsbedingung sogar besser als elastisch bemessene Tragwerke. 


\section{Riassunto e conclusioni}

Nel presente lavoro si analizza l'applicabilità della teoria della plasticità nel dimensionamento di strutture in cemento armato $e$ in cemento armato precompresso. Si cerca cioè di dare una risposta al quesito seguente: "Si puó determinare mediante la teoria della plasticità il carico limite ultimo di un sistema iperstatico con sufficiente precisione e si comporta una struttura cosi dimensionata in modo accettabile allo stato di servizio?" In questa domanda sono presenti due aspetti: il comportamento allo stato limite ultimo e allo stato di servizio. Questi aspetti verranno trattati separatamente.

II carico limite ultimo di un sistema iperstatico puó essere sopravvalutato 0 anche sottovalutato applicando la teoria della plasticità. Questo accade quando l'ipotesi di un comportamento perfettamente plastico non corrisponde a realtà o (oppure e) quando spostamenti importanti vengono tralasciati nella formulazione delle equazioni d'equilibrio. In questo lavoro si considerano solamente gli effetti per i quali la teoria della plasticità porta a una sopravvalutazione del carico limite ultimo. Ciò accade nel caso di "softening" e di effetti del secondo ordine in presenza di forze di compressione.

Si parla di "softening" quando al crescere delle deformazioni la resistenza diminuisce. Nel caso di un sistema questo accade in modo repentino quando l'energia accumulata fino al raggiungimento del carico limite ultimo (deformazioni elastiche) è maggiore dell'energia che puó essere dissipata durante la rottura (energia plastica). Dato che l'energia elastica accumulata è funzione del tipo di sistema, delle sue dimensioni e di come esso è sollecitato, questi fattori influiscono sulla fragilità del sistema in misura uguale alle caratteristiche meccaniche del materiale. Questo puó essere quantificato con il cosiddetto fattore di instabilità da "softening" che è definito come rapporto fra energia accumulata e energia dissipabile.

Non solo la fragilità del materiale provoca effetti di "sottening" nella struttura, essi possono anche risultare da effetti del secondo ordine.

II comportamento meccanico dell'acciaio, del calcestruzzo e del cemento armato viene trattato riportando innumerevoli esperienze descritte nella letteratura scientifica. Con particolare cura viene trattata la capacità di dissipare energia durante la rottura. Si mostra come questa energia può essere aumentata con accorgimenti costruttivi come l'impiego di armatura trasversale e armatura di compressione sufficientemente stabilizzata dalle staffe.

Dall'analisi di un semplice sistema iperstatico composto di due aste sollecitate in parallelo risulta come il carico limite ultimo viene influenzato dal fenomeno di "softening" dell'asta nella quale per primo si raggiunge la resistenza, mentre quella dell'altra asta influisce sul comportamento del sistema ma non sul suo carico limite ultimo. In generale per fare in modo che le resistenze dei singoli sottosistemi possano essere sfruttate al meglio è opportuno attivare dapprima quelli con un comportamento duttile e, solo dopo essere giunti alla loro resistenza, attivare i sottosistemi fragili. Se il sistema è composto di molti elementi fragili bisognerà fare in modo che essi raggiungano la loro resistenza contemporaneamente. 
Quale esempio rappresentativo di sistema nel quale le deformazioni plastiche avvengono in zone sollecitate prevalentemente a flessione si analizza una trave incastrata alle due estremità. II suo carico limite ultimo viene influenzato dalle autotensioni presenti prima che il carico venga applicato (esse influenzano infatti la ridistribuzione plastica degli sforzi e quindi la diminuzione del carico limite ultimo dovuta a "softening"), dalla snellezza della trave (aumentando la luce della trave lasciando invariata la sua sezione si aumenta la quantità di energia accumulata ma non di quella che puó essere dissipata cosicché aumenta la fragilità del sistema) e dalle caratteristiche della sezione (energia dissipabile). Si dimostra come nel caso di un'armatura trasversale efficiente nella zona compressa si abbia un comportamento duttile della trave e come il carico limite ultimo possa essere predetto con sufficiente precisione con la teoria della plasticità.

Si analizzano anche alcuni sistemi nei quali le deformazioni plastiche avvengono in elementi compressi o pressoinflessi. Le conclusioni che scaturiscono da queste analisi non possono però essere generalizzate data la grande varietà di strutture di questo tipo.

Nel caso di sistemi con deformazioni plastiche in elementi sollecitati soprattutto a taglio con la conseguente frattura del calcestruzzo nell'anima, l'energia dissipata è molto inferiore a quella accumulata di modo che si assiste di solito ad una diminuzione di carico (resistenza della struttura) dopo il formarsi di una cerniera plastica di questo tipo. In questi casi la teoria della plasticità è applicabile solo a determinate condizioni.

Nel presente lavoro si mostra come la teoria della plasticità possa essere applicata nel dimensionamento di sistemi composti di elementi sia fragili che duttili. Si descrive un metodo di dimensionamento mediante il quale solo gli elementi duttili risultano determinanti. Si possono cosi dimensionare in modo semplice strutture anche particolarmente complesse ottenendo un comportamento duttile allo stato limite ultimo.

Alcuni elementi di strutture in cemento armato caratterizzati da un'insufficiente armatura trasversale presentano allo stato limito ultimo fessure di tale ampiezza da poter pregiudicare la trasmissione di sforzi attraverso tali fessure mediante ingranamento. Si analizzano alcuni casi di questo tipo quali travi e colonne senza armatura al taglio e solai appoggiati direttamente su pilastri (punzonamento). In questi casi l'applicazione della teoria della plasticità conduce ad una soprawalutazione del carico limite ultimo. II confronto delle soluzioni plastiche con le sollecitazioni che effettivamente si instaurano permette di chiarire le cause del collasso precoce e di sviluppare accorgimenti costruttivi adeguati. II carico limite ultimo previsto secondo la teoria della plasticità puó essere raggiunto se si influenza in modo opportuno il quadro fessurativo. Questo può essere raggiunto mediante autotensioni (per esempio precomprimendo), dando una forma adeguata all'elemento portante oppure con un'armatura di ripartizione sufficiente. In questo modo si ottiene anche un comportamento duttile allo stato limite ultimo.

Da ultimo si verifica se sistemi dimensionati con il metodo plastico abbiano un comportamento accettabile allo stato di servizio. In sistemi iperstatici si formano solitamente cerniere plastiche prima del raggiungimento del carico limite ultimo. Se questo avviene allo stato di servizio larghe fessure in corrispondenza delle cerniere plastiche possono compromettere la 
qualità della struttura. Si noti però che la formazione di cerniere plastiche e la conseguente ridistribuzione plastica degli storzi non avviene solo nel caso di sistemi dimensionati sulla base della plasticità. Ciò accade anche in strutture dimensionate con il metodo elastico poiché la caratteristica sforzo-deformazione viene di solito troppo drasticamente semplificata. Strutture dimensionate con il metodo plastico possono essere allo stato di servizio qualitativamente migliori 0 peggiori della medesima struttura dimensionata con il metodo elastico. Ció dipende da come vengono ripartite le resistenze fra i sottosistemi componenti la struttura. Si posticipa la formazione delle prima cemiera plastica, migliorando cosi il comportamente della struttura, facendo in modo che la resistenza dei sottosistemi, nei quali avviene dapprima la formazione di cerniere plastiche, sia maggiore di quella degli altri sottosistemi. Questo si ottiene armando soprattutto le zone della struttura dove le deformazioni specifiche sono maggiori (cioè dove si raggiunge per primo lo snervamento dell'armatura). Un sistema dimensionato con un metodo plastico facendo in modo che questa condizione supplementare venga soddidfatta, avrà solitamente un comportamento migliore allo stato di servizio della medesima struttura dimensionata con un metodo elastico. 


\section{Summary and conclusions}

An investigation of whether the theory of plasticity can be used in the design of reinforced and prestressed concrete is presented in this dissertation. Answers to the question, "Can the degree of safety be reliably estimated and is the behaviour under service conditions satisfactory in a structural system designed using plastic methods?" are sought through separate investigations of structural behaviour at ultimate limit state and under service conditions.

The theory of plasticity can overestimate or underestimate the ultimate load of statically indeterminate systems when the assumption of ideal plastic behaviour is unrealistic or when large deformations are neglected in the formulation of the equations of equilibrium. Only those deviations from the theory of plasticity that result in a reduction of the ultimate load are considered in this dissertation, namely, softening behaviour and second-order effects in compression members.

Softening is reduction in resistance with increasing deformations after material strength has been reached. The softening of a system occurs rapidly when the strain energy stored by elastic deformation up to ultimate load is large relative to the energy dissipated by plastic deformation. Since the energy stored is a function of the type and size of the system and the type of loading, these factors influence the brittleness of the system in addition to material properties. They can be described with a softening stability factor, which is defined as the ratio of the stored energy to the dissipated energy. Softening of the system is produced by both strain softening of the material and second-order effects.

The mechanical behaviour of steel, concrete, and reinforced concrete is described with the interpretation of numerous studies. The capacity to dissipate energy through plastic deformation is discussed in particular detail. The amount of energy that can be dissipated is favourably influenced by structural details such as appropriate transverse reinforcement and the proper confinement of compression reinforcement.

A system that is statically indeterminate to the first degree, consisting of two coupled subsystems, is used to demonstrate that the ultimate load is influenced by the softening characteristics of the subsystem in which plastification first occurs. The softening of the subsystem that plastifies last influences the behaviour of the system after the ultimate load is reached, but never the ultimate load itself. To allow the resistances of individual subsystems to be utilized as much as possible, therefore, the system should be designed to ensure that the ductile subsystems plastify before the brittle subsystems. When several brittle subsystems are present, their resistances should be activated simultaneously.

A fixed-end beam is investigated as a representative example of systems that deform plastically by means of flexural hinges. The ultimate load is influenced by self-equilibrating stresses (large or small reduction of ultimate load due to softening, depending on self-equilibrating stresses), by beam slenderness (brittleness increases with slenderness), and by the design of the cross-section (energy that can be dissipated). The behaviour of the system is ductile when effective transverse reinforcement in the compression zone is provided; the ultimate load can be 
calculated with good accuracy, independently of self-equilibrating stresses and for high values of slenderness, using the theory of plasticity.

Several examples of subsystems exhibiting plastic deformations in compression members are treated. No generally valid conclusions can be drawn due to the wide variety possible in such systems.

In systems with plastic deformations in shear hinges with concrete failure in the webs, the energy that can be dissipated is so small relative to the energy that is normally stored elastically. Softening of the system often follows after shear strength is reached. The use of the theory of plasticity is somewhat problematic in these cases.

The theory of plasticity can nevertheless be used successfully, even in systems with brittle components. These components can be designed using plastic methods to ensure that they do not become critical. Such a design procedure, which is proposed in this dissertation, simplifies the design of complex systems.

Wide cracks can occur in reinforced concrete components that have insufficient transverse reinforcement. This can result in an insufficient transfer of force due to the lack of interlocking at the crack faces. Several such cases are treated, for example beams and compression members without shear reinforcement and slabs in the vicinity of columns. In these cases, the use of the theory of plasticity leads to an overestimate of the ultimate load. A comparison of the structural behaviour proposed according to this theory and the behaviour that actually occurs enables the cause of failure to be recognized and appropriate solutions to be detailed.

The structural behaviour that corresponds to the theoretical model of plasticity can occur if the crack pattern can be favourably influenced with an appropriate self-equilibrating state of stress (e.g. prestressing), a sensible shape for the beam, and above all with crack distribution reinforcement. In addition to ductile behaviour, these measures enable the ultimate load computed from plasticity theory to be reached.

Finally, the behaviour under service conditions of structural systems that have been designed using the theory of plasticity is treated. In statically indeterminate systems, initial plastification occurs before the ultimate load is reached. If this occurs at service load levels, wide cracks can be produced and the serviceability of the structure impaired. The formation of plastic hinges and the redistribution of internal forces occur not only in structures that have been designed using plastic methods but also in structures that have been designed elastically, since the actual load-deformation behaviour of reinforced concrete is normally not considered. The behaviour of plastically designed systems can be better or worse than that of elastically designed systems, depending on the choice of resistances. The greater the load that produces the first plastic hinge, the better is the behaviour of the system under service conditions. This is the case when the subsystems that yield first resist a large portion of the ultimate load. This implies that the structural components that are characterized by large specific deformations, and thus yield first, should be the most strongly reinforced. Systems designed in this way satisfy serviceability criteria even better than elastically designed systems. 


\section{Literaturverzeichnis}

Ahmad S.H., Shah S.P. (1982): "Stress-Strain Curves of Concrete Confined by Spiral Reinforcement",ACI-Journal, November/December 1982, 484-490.

Ahmad S.H., Shah S.P. (1985): "Structural Properties of High Strength Concrete and its Implications for Precast Prestressed Concrete", PCl-Journal, November/December 1985, 92-119.

Bach F., Nielsen M.P., Braestrup M.W. (1980): "Shear Tests on Reinforced Concrete T-Beams, Series $\mathrm{V}, \mathrm{U}, \mathrm{X}, \mathrm{B}$ and S", Technical University of Denmark, Structural Research Laboratory, Report No.R 120, Copenhagen 1980, $86 \mathrm{pp}$.

Base G.D., Read J.B. (1965): "Effectiveness of Helical Binding in the Compression Zone of Concrete Beams", ACI-Journal, July 1965, 763-781.

Bazant Z.P. (1976): "Instability, Ductility and Size Effect in Strain-Softening Concrete", Journal of the Engineering Mechanics Division, ASCE, Vol. 102, No. EM 2, April 1976, 331-344.

Bennet E.W., Balasooriya B.M.A (1971): "Shear Strength of Prestressed Beams with Thin Webs Failing in Inclined Compression", ACl-Journal, March 1971, 204-212.

Bertero V.V., Felippa C. (1964): Diskussion zum Artikel von Roy H.E.H., Sozen M.A. "Ductility of Concrete", Flexural Mechanics of Reinforced Concrete, Proc. of the Internat. Symposium ASCE-ACI, Miami 1964, 227-235.

Bollinger K. (1985): "Zu Tragverhalten und Bewehrung von rotationssymmetrisch beanspruchten Stahlbetonplatten", Universităt Dortmund, Dissertation, $257 \mathrm{p}$.

Braestrup M.W., Nielsen M.P., Bach F., Jensen B.C. (1976): "Shear Tests on Reinforced Concrete TBeams, Serie T", Technical University of Denmark, Structural Research Laboratory, Report No. R 72, Copenhagen 1976, $114 \mathrm{pp}$.

Bresler B., Gilbert P.H. (1961): "Tie Requirements for Reinforced Concrete Columns", ACI-Journal, November 1961, 555-569.

Burdette E.G., Hilsdort H.K. (1971): "Behaviour of Laterally Reinforced Concrete Columns", ASCE, Journal of the Structural Division, Vol. 97, No. ST2, February 1971, 587-602.

CEB-FIP (1978): "Code modèle pour les structures en béton", Système international de réglementation technique unifiée des structures, Bulletin d'information No.125, Paris 1978, 336 pp.

Cedolin L., Dei Poli S., lori I. (1981): "Analisi sperimentale del processo di formazione della frattura nel calcestruzzo", Studi e Ricerche, Corso di perfezionamento per le costruzioni in cemento armato F.lli Pesenti, Politecnico di Milano, Vol. 3, 1981, 47-74. 
Cedolin L., Dei Poli S., lori I. (1983): "Comportamento a trazione del calestuzzo", Studi e Ricerche, Corso di perfezionamento per le costruzioni in cemento armato F.lli Pesenti, Politecnico di Milano, Vol. 5, 1983, 23-46.

Cedolin L., Dei Poli S., lori I. (1986): "Determinazione indiretta del legame costitutivo del calcestruzzo sotto stati tensionali di trazione", Studi e Richerche, Corso di perfezionalmento per le costruzioni in cemento armato F.lli Pesenti, Politecnico di Milano, Vol. 8, 1986, 9-62.

Chen W.F. (1975): "Limit Analysis and Soil Plasticity", Elsevier Scientific Publishing Company, Amsterdam, Oxford, New York, 1975, 638 pp.

Cranston W.B. (1965): "Tests on Reinforced Concrete Frames; Part 1, Pinned Portal Frames",Technical Report No. TRA392, Cement and Concrete Association, London 1965, 38 pp.

Darvall P.L. (1983): "Critical Softening of Hinges in Indeterminate Beams and Portal Frames", Transactions of the Institution of Engineers, Australia, Civil Engineering, Vol. CE 25, No. 3, August 1983, 199-210.

De Donato O. (1969): "Indagine sperimentale sul comportamento a collasso ed in regime instabile di elementi tubolari compressi", Rendiconti dell' Istituto Lombardo di Scienza e Lettere, Vol. A 103, Milano, 1969, 119-144.

Drucker D.C. (1961): "On Structural Concrete and the Theorems of Limit Analysis", Internat. Vereinigung für Brückenbau und Hochbau, Zürich, IVBH-Abhandlungen 21, 1961, 49-59.

Gvozdev A.A. (1949): "Raschet nesushchei spasobnosti Konstruktsii po metodu pre delnogo ravnoveiya", Moskau, Stroiizdat, 1949, 280 pp.

Hagberg T. (1967): "Versuche an indirekt gelagerten Plattenbalken", Technische Hochschule Norwegen, Institut für Beton und Betonkonstruktionen, Trondheim, Februar 1967, 89 pp.

Hamadi Y.D., Regan P.E. (1980): "Behaviour in Shear of Beams with Flexural Cracks", Magazine of Concrete Research, Vol. 32, No. 111, June 1980, 67-78.

Han D.J., Chen W.F. (1983): "Behaviour of Portal and Strut Types of Beam-Columns", Eng. Struct., Vol. 5, January 1983, 180-190.

Hennebique F. (1893): "Poutre légère et de grande résistance en béton de ciment avec barres de fer noyées", Schweizerisches Patent Nr. 6533, Klasse 5, Bern, 1893.

Hennebique F. (1897): "Système de poutre en béton armé", Schweizerisches Patent Nr. 15977, Klasse 5, Bern 1897.

Hillerborg A. (1983): "Analysis of one single Crack", Fracture Mechanics of Concrete, Ed. Wittmann, Elsevier, Amsterdam 1983, 223-250. 
Iqbal M., Hatcher D.S. (1975): "Post-Crushing Behaviour of Unbounded Concrete Beams", ASCE, Journal of the Structural Division, Vol. 101, No. ST11, November 1975, 2303-2316.

Ivanyi G., Buschmeyer W. (1981): "Biegeversuche an Plattenstreifen mit zentrischer Vorspannung ohne Verbund", Gesamthochschule Essen, Forschungsberichte aus dem Fachbereich Bauwesen, Heft Nr. 16, Mai 1981, 75 pp.

Jaccoud J.-R., Francou B., Camara J.-M. (1984): "Armature minimale pour le contrôle de la fissuration", Institut de statique et structures (béton armé et précontraint), EPF Lausanne, mai 1984, 69 pp.

Jensen J.F. (1981): "Plasticitetsteoretiske Losninger for Skiver og Bjaelker af Jernbeton", Afdelingen for Bearende Konstruktioner, Danmarks Tekniske Hojskole, Report No. 141, 153 pp.

Johansen K.W. (1943): "Bruchlinieteorier" (Yield line theories), Kopenhagen, Gjellerup, 1943, 189 pp.

Kanellopoulos A. (1986): "Zum unelastischen Verhalten und Bruch von Stahlbeton", Institut für Baustatik und Konstruktion, ETH Zürich, Bericht Nr. 153, 1986, Birkhăuser Verlag AG, Basel, 86 pp.

Kani G. (1955): "Spannbeton in Entwurf und Austührung", Verlag Konrad Wittwer, Stuttgart, 1955, 501-531.

Kani G. (1964): "The Riddle of Shear Failure and Its Solution", Journal of the American Concrete Institute, Vol. 61, No. 4, 1964, 441-467.

Kinnunen S., Nylander H. (1960): "Punching of Concrete Slabs Without Shear Reinforcement", Transactions of the Royal Institute of Technology, Stockholm, No. 158, 112 pp.

Kosaka Y., Tanigawa Y., Hatanaka S. (1984): "Inelastic Deformational Behaviour of Axially Loaded Concrete under Low Lateral Confining Stress", Transactions of the Japan Concrete Institute, Vol. 6, 1984, 263-270.

Kosaka Y., Tanigawa Y., Hatanaka S. (1985): "Evaluation of Effect of Confinements on Compressive Toughness of Concrete Based on Triaxial Compressive Test Data", Transactions of the Japan Concrete Institute, Vol. 7, 1985, 249-256.

Kupfer H. (1973): "Das Verhalten des Betons unter mehrachsiger Kurzzeitbelastung unter besonderer Berücksichtigung der zweiachsigen Beanspruchung", Deutscher Ausschuss für Stahlbeton, Heft 229, Verlag W. Ernst \& Sohn, Berlin 1973.

Lampert P. (1970): "Bruchwiderstand von Stahlbetonbalken unter Torsion und Biegung", Institut für Baustatik und Konstruktion, ETH Zürich, Bericht Nr. 26 (Dissertation), Birkhäuser Verlag, Basel, $189 \mathrm{pp}$. 
Leonhardt F., Walther R. (1962a): "Schubversuche an einfeldrigen Stahlbetonbalken mit und ohne Schubbewehrung", Deutscher Ausschuss für Stahlbeton, Heft 151, 83 pp.

Leonhardt F., Walther R. (1962b): "Versuche an Plattenbalken mit hoher Beanspruchung", Deutscher Ausschuss für Stahlbeton, Heft 152, 71 pp.

Leonhardt F., Walther R. (1963): "Schubversuche an Plattenbalken mit unterschiedlicher Schubbewehrung", Deutscher Ausschuss für Stahlbeton, Heft 156, 84 pp.

Lyngberg B.S. (1976): "Ultimate Shear Resistance of Partially Prestressed Reinforced Concrete 1Beams", ACl-Journal, April 1976, 214-222.

Maier G. (1968): "Sul comportamento flessionale instabile nelle travi inflesse elasto-plastiche", Istituto Lombardo, Accademia di scienze e lettere, Rendiconti, Scienze matematiche, fisiche, chimiche e geologiche, Vol. A102 (1968), 648-677. Engl. Version: "On Softening Flexural Behaviour in Elastic-Plastic Beams", Corso di perfezionamento per le costruzioni in cemento armato F.lli Pesenti, Politecnico di Milano, Studi e Ricerche, Vol. 8 (1986), 85-117.

Marti P. (1980): "Zur plastischen Berechnung von Stahlbeton", Institut für Baustatik und Konstruktion, ETH Zürich, Bericht Nr. 104 (Dissertation), Oktober 1980, Birkhăuser Verlag, Basel, 176 pp.

Menne B (1977): "Zur Traglast der ausmittig gedrückten Stahlbetonstütze mit Umschnürbewehrung", Deutscher Ausschuss für Stahlbeton, Heft 285, Verlag W. Ernst \& Sohn, Berlin 1977, 56 pp.

Moehle J.P., Cavanagh T. (1985): "Confinement Effectiveness of Crossties in RC", ASCE, Journal of the Structural Division, Vol. 111, No. ST10, October 1985, 2105-2120.

Mörsch E. (1908): "Der Eisenbetonbauer, seine Theorie und Anwendung, Verlag von Konrad Wittwer, Stuttgart, 3. Auflage.

Mörsch E. (1929): "Der Eisenbetonbauer, seine Theorie und Anwendung", Verlag von Konrad Wittwer, Stuttgart, 1. Band, 2. Hälfte, 6. Auflage, $541 \mathrm{pp}$.

Müller P. (1978): "Plastische Berechnung von Stahlbetonscheiben und -balken", Institut für Baustatik und Konstruktion, ETH Zürich, Bericht Nr. 83 (Dissertation), Birkhăuser Verlag, Basel, 160 pp.

Müller F.-X., Muttoni A., Thürlimann B. (1984): "Durchstanzversuche an Flachdecken mit Aussparungen", Institut für Baustatik und Konstruktion, ETH Zürich, Versuchsbericht Nr. 7305-5, Birkhäuser Verlag, Basel, $118 \mathrm{pp}$.

Muttoni A., Thürlimann B. (1986): "Schubversuche an Balken und Flachdecken ohne Schubbewehrung", Institut für Baustatik und Konstruktion, ETH Zürich, 1986, unveroffentlichter Bericht.

Muttoni A., Schwartz J., Thürlimann B. (1987): "Bemessen und Konstruieren von Stahlbetontragwerken mit Spannungsfeldern", Institut für Baustatik und Konstruktion, ETH Zürich, Vorlesungsunterlagen, Sommersemester 1987, 113 pp. 
Nielsen M.P. (1984): "Limit Analysis and Concrete Plasticity", Prentice Hall, Englewood Cliffs, New Jersey, $420 \mathrm{pp}$.

Ottosen N.S. (1986): "Thermodynamic Consequences of Strain Softening in Tension", ASCE, Journal of Engineering Mechanics, Vol. 112, No. 11, November 1986, 1152-1164.

Phipps M.E. (1976): "The strain capacity of compression-zone concrete subjected to short-term loading", Magazine of Concrete Research, Vol. 28, No. 95, June 1976, 85-100.

Prager W., Hodge P.G. (1951): "Theory of perfectly plastic solids", John Wiley \& Sons, New York, $1951,264 \mathrm{pp}$.

Prager W. (1955): "Probleme der Plastizitătstheorie", Birkhăuser Verlag AG Basel, 1955, 100 pp.

Regan P. (1971): "Shear in Reinforced Concrete, an Experimental Study", Imperial College, Civil Engineering Dept., Concrete Section, London, April 1971, 203 pp.

Reineck K.-H. (1982): "Models for Design of Reinforced and Prestressed Concrete Members", Comité Euro-International du Béton (CEB), Bulletin d'information No. 146, 43-96.

Reinhardt H.W. (1984): "Fracture Mechanics of an Elastic Softening Material like Concrete", Heron, Vol. 29 (1984), No. 2, 42 pp.

Richart F.E., Brandtzaeg A., Brown R.L. (1928): "A Study of the Failure of Concrete under Combined Compressive Stresses", University of Illinois, Engineering Experiment Station, Bulletin 185, November 1928, $102 \mathrm{pp}$.

Richart F.E., Brandtzaeg A., Brown R.L. (1929): "The Failure of Plain and Spirally Reinforced Concrete in Compression", University of Illinois, Engineering Experiment Station, Bulletin 190, April 1929, 72 pp.

Ritter W. (1899): "Die Bauweise Hennebique", Schweizerische Bauzeitung, Vol. 17 (1899), 41-43, 4952 und $59-61$.

Ritz P., Marti P., Thürlimann B. (1975): "Versuche über das Biegeverhalten von vorgespannten Platten ohne Verbund", Institut für Baustatik und Konstruktion, ETH Zürich, Versuchsbericht Nr. 73051, Juni 1975, Birkhăuser Verlag AG, Basel, 114 pp.

Robinson J.R. (1961): "Essais à l'effort tranchant de poutres à åme mince en béton armé", Annales des Ponts et Chaussées, 131e année, No. 2, Paris, mai-avril 1961, 225-255.

Robinson J.R., Demorieux J.-M. (1976): "Essais de poutres en double Té en béton armé", Annales de I'Institut Technique du Bâtiment et des Travaux Publics, No. 335, Paris, Janvier 1976, 66-91. 
Schlaich J., Weischede D. (1982): "Ein praktisches Verfahren zum methodischen Bemessen und Konstruieren im Stahlbetonbau", Comité Euro-International du Béton (CEB), Bulletin d'information, No. 150, Paris, 1982, $163 \mathrm{pp}$.

Schreyer H.L., Chen Z. (1986): "One-Dimensional Softening with Localisation", Journal of Applied Mechanics, ASME, Vol. 53, December 1986, 791-797.

Sheikh S.A., Uzumeri S.M. (1980): "Strength and Ductility of Tied Concrete Columns", ASCE, Journal of the Structural Division, Vol. 106, No. ST5, May 1980, 1079-1102.

Sherman D.R. (1980): "Post-Local Buckling Behaviour of Tubular Strut Type Beam-Columns, an Experimental Study", Report to Shell Oil Company, University of Wisconsin-Milwaukee, June 1980.

Somes N.F. (1970): "Compression Tests on Hoop-Reinforced Concrete", ASCE, Journal of the Structural Division, Vol. 96, No. ST7, July 1970, 1495-1509.

Sorensen H.C. (1974): "Shear Tests on 12 Reinforced Concrete T-Beams", Technical University of Denmark, Struct. Research Lab., Report No. R60, Copenhagen, 1974, 49 pp.

Sozen M.A., Zwoyer E.M., Siess C.P. (1959): Strength in Shear of Beams Without Web Reinforcement", University of lllinois, Engineering Experiment Station, Bulletin No. 452, 1959, 69 pp.

Tanigawa Y., Yamada K. (1981): "Analyses of Stress-Strain Curves of Various Concretes by Endochronic Theory", Transactions of the Japan Concrete Institute, Vol. 3, 1981, 179-186.

Taylor R. (1966): "Some shear tests on reinforced concrete T-beams with stirrups", Magazine of Concrete Research, Vol. 18, No. 57, December 1966, 221-230.

Thürlimann B. (1959): "New Aspects Concerning Inelastic Instability of Steel Structures", Lehigh University, Fritz Engineering Laboratory, Report No. 205.66, Bethlehem, July 1959, 46 pp.

Thürlimann B., Grob J., Lüchinger P. (1975): "Torsion, Biegung und Schub in Stahlbetontrăgern", Institut für Baustatik und Konstruktion, ETH Zürich, Autographie zum Fortbildungskurs für Bauingenieure aus der Praxis, 170 pp.

Thürlimann B., Marti P., Pralong J., Ritz P., Zimmerli B. (1983): "Anwendung der Plastizitătstheorie auf Stahlbeton", Institut für Baustatik und Konstruktion, ETH Zürich, Autographie zum Fortbildungskurs für Bauingenieure aus der Praxis, April 1983, 252 pp.

Tomii M., Sakino K., Kiyohara K. (1981): "Experimental Studies on Plain Concrete Column Subjected to Monotonic Shearing Force and Constant Axial Force", Transactions of the Architectural Institute of Japan, No. 307, September 1981, 46-55.

Van Mier J.G.M. (1984): "Strain-Softening of Concrete under Multiaxial Loading Conditions", thesis, Technical University Eindhoven, 1984, 349 pp. 
Vecchio F., Collins M.P. (1982): "The Response of Reinforced Concrete to In-Plane Shear and Normal Stresses", University of Toronto, Dept. of Civil Engineering, Publication No. 82-03, March 1982, $332 \mathrm{pp}$.

Vecchio F., Collins M.P. (1986): "The Modified Compression-Field Theory for Reinforced Concrete Elements Subjected to Shear", Journal of the American Concrete Institute, Proceedings, Vol. 83, No. 2, March-April 1986, 219-231.

Walraven J.C. (1981): "The Behaviour of Cracks in Plain and Reinforced Concrete Subjected to Shear", IABSE-Colloquium Delft 1981, Final Report, 227-244.

Wang P.T., Shah S.P., Naaman A.E. (1978): "Stress-Strain Curves of Normal and Lightweight Concrete in Compression", Journal of the American Concrete Institute, Proceedings, Vol. 75, No. 11, November 1978, 603-611.

Wischers G. (1978): "Aufnahme und Auswirkungen von Druckbeanspruchungen auf Beton", Beton, Heft Nr. 2, 1978, 63-67.

Yashiro H., Hanai S., Takahashi K. (1984): "Effect of Transverse Reinforcement on Strength and Ductility of Tied Concrete Columns", Transactions of the Japan Concrete Institute, Vol. 6, 1984, 703-710.

Yashiro H., Hanai S., Takahashi K. (1986): "Experimental Study of Transversal Reinforcement on Ductility of Tied Concrete Columns", Transactions of the Japan Concrete Institute, Vol. 8, 1986, 527-534. 


\section{Bezeichnungen}

a

d

e

f

g

h

l

m

n

9

$\mathbf{s}$

$\mathbf{t}$

$\mathbf{u}$

V

w

$x$

$\begin{array}{ll}\text { A } & \text { Flăche; Arbeit } \\ \text { D } & \text { Entfestigungsmodul } \\ \text { E } & \text { Elastizitătsmodul } \\ \text { G } & \text { Schubmodul } \\ \text { I } & \text { Trăgheitsmoment } \\ \text { K } & \text { Steifigkeit } \\ \text { M } & \text { Moment } \\ \text { N } & \text { Normalkraft } \\ \text { P } & \text { Vorspannkraft } \\ \text { Q } & \text { Last } \\ \text { T } & \text { Torsionsmoment; Temperatur } \\ \text { V } & \text { Querkraft } \\ \text { W } & \text { Energie }\end{array}$

Schubspannweite

statische Hohe, Breite, Durchmesser

Exzentrizităt

Festigkeit

Eigengewicht

Hơhe

Lảnge

verteilte Last

Bügelabstand

Scheibendicke, Stegdicke

Verlăngenung

Verschiebung

Koordinate

Koordinate; Hebelarm

dimensionsloses Moment $m=M / t \cdot h^{2} \cdot t_{c}$

Verhăltnis $E_{S t a h l} / E_{\text {Beton }}$; dimensionslose Normalkraft $n=N / t \cdot h \cdot f_{C}$

Verschiebung; dimensionslose Querkraft $v=V / t \cdot h \cdot f_{c}$

Abmessung der Druckzone; Koordinate 
$\alpha \quad$ Winkel; Faktor; Schubschlankheit

B Faktor

$\gamma \quad$ Schiebung; Sicherheitsfaktor

$\varepsilon \quad$ Dehnung

$\zeta \quad$ dimensionslose Durchbiegung

$\eta \quad$ dimensionslose Last

$\vartheta \quad$ Druckfeldneigung

к Krümmung

$\lambda$ Schlankheit

$\mu \quad$ Momentenverhältnis

$\checkmark \quad$ dimensionslose Normalkraft

$\xi$ dimensionslose Verkürzung; dimensionslose Hőhe der Druckzone

$\rho \quad$ geometrischer Bewehrungsgehalt

$\sigma \quad$ Normalspaninung

$\tau \quad$ Schubspannung

$\varphi \quad$ Rotationswinkel

$\chi \quad$ Entfestigungsstabilitătsfaktor

$\psi \quad$ Faktor $\mathrm{h} / \mathrm{d}$; dimensionslose Zwängung

$\omega \quad$ mechanischer Bewehrungsgehalt

$\Phi \quad$ Durchmesser eines Bewehrungsstabes

Indizes

$\begin{array}{ll}\text { C } & \text { Beton } \\ \text { cr } & \text { kritisch } \\ \text { dir } & \text { Direktabstützung } \\ \text { flex } & \text { Biegetraglast (Traglast nach der Plastizitătstheorie) } \\ \text { h } & \text { Verfestigung } \\ \ell & \text { lănger } \\ r & \text { Riss } \\ \text { R } & \text { Widerstand; Bruch } \\ \text { s } & \text { Entfestigung; Stahl } \\ \text { t } & \text { Zug, quer } \\ \text { y } & \text { Fliessen } \\ \text { z } & \text { Zwăngung } \\ \text { zul } & \text { zulăssig }\end{array}$




\section{Anhang 1: Auswertung von Balkenschubversuchen}

Die in der Tabelle A 1.1 angegebenen Serien von Versuchen an einfachen Balken mit vertikaler Schubbewehrung werden ausgewertet. Die Daten der 216 Versuche sind in der Tabelle A1.2 enthalten. Bild A 1.1 zeigt die Bedeutung der verwendeten Bezeichnungen.

\begin{tabular}{|c|c|c|}
\hline $\begin{array}{l}\text { Ort der } \\
\text { Versuchsdurchführung }\end{array}$ & Bezeichnung & $\begin{array}{l}\text { Versuchsberichte } \\
\text { (siehe Literaturverzeichnis) }\end{array}$ \\
\hline Kopenhagen & $\begin{array}{l}\text { KV.... } \square \\
K U . . . \\
K X \ldots \\
K B \ldots . . \\
K R . . .\end{array}$ & Bach, Nielsen, Braestrup (1980) \\
\hline Kopenhagen & KT... $\triangle$ & Braestrup, Nielsen, Bach (1976) \\
\hline Kopenhagen & кт... $\nabla$ & Sorensen (1974) \\
\hline London & LS.... & Taylor (1966) \\
\hline Trondheim & TLB. $\triangle$ & Hagberg (1967) \\
\hline Paris & PBQ.. \lceil & Robinson, Demorieux (1976) \\
\hline London & $\begin{array}{l}\text { LR... X } \\
\text { LJ... } \\
\text { LT... } \\
\text { LW... }\end{array}$ & Regan (1971) \\
\hline Stuttgart & ST1 + & Leonhardt, Walther (1962b) \\
\hline Stuttgart & SET.. & Leonhardt, Walther (1962a) \\
\hline Stuttgart & $\begin{array}{l}\text { STA.. } \\
\text { SETI }\end{array}$ & Leonhardt, Walther (1963 \\
\hline Kopenhagen & $K A \ldots \diamond$ & Lyngberg (1976) \\
\hline Leeds & LE... & Bennet, Balasooriya (1971) \\
\hline Paris & PN... Y & Robinson (1961) \\
\hline
\end{tabular}

Tabelle A 1.1: Ausgewertete Versuchsserien
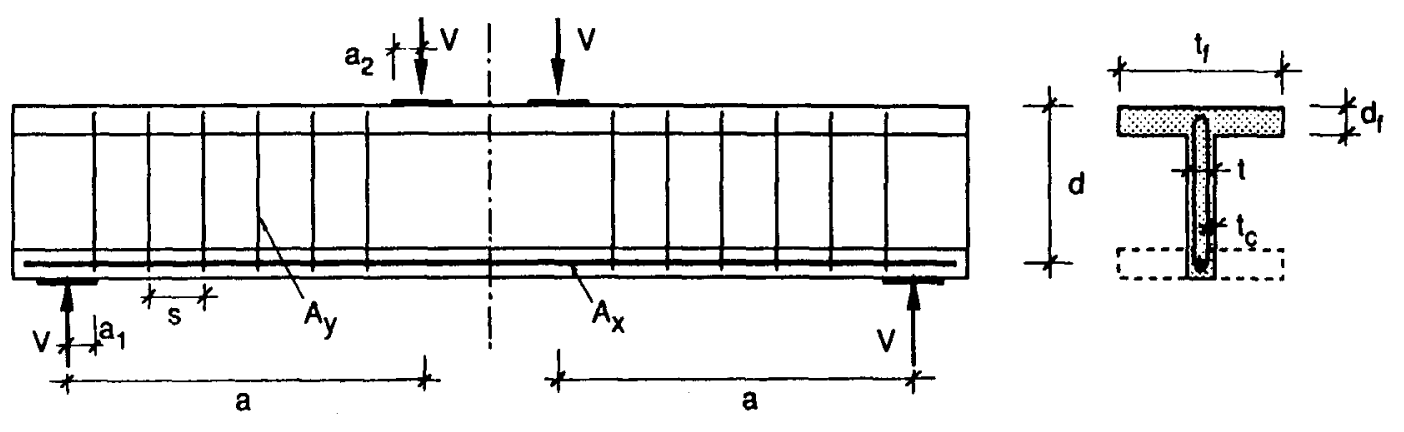

Bild A 1.1: Balkengeometrie mit Bezeichnungen 
Für die Materialeigenschaften gilt:

$f_{c}=$ Zylinderfestigkeit [MPa]; (wenn im Versuchsbericht nur die Würfeldruckfestigkeit angegeben ist, dann wird mit $f_{c}=0.8 f_{c W}$ gerechnet)

$\mathrm{f}_{\text {syx }}=$ Streckgrenze der Längsbewehrung $[\mathrm{MPa}]$

$f_{\text {syy }}=$ Streckgrenze der Schubbewehrung [MPa]

Die Zielsetzung dieser Versuchsauswertung besteht darin, die experimentell ermittelte Festigkeit der schubbeanspruchten Zone (Steg) mit den theoretischen Werten zu vergleichen. Aus diesem Grund werden zuerst diejenigen Versuche ausgeschlossen, bei welchen die Bruchlast infolge Fliessens der Längsbewehrung im mittleren Bereich ohne Schubbeanspruchung (Biegemechanismus) erreicht wird. Um dies mit genügender Sicherheit ausführen zu können, werden die Versuche aussortiert, die die Bedingung $V \leq 0.95 \cdot V_{\text {flex }}$ nicht erfüllen (Tabelle $A$ 1.3). $V_{\text {flex }}$ ist die plastisch berechnete Biegetraglast. In der Druckzone wird mit einer konstanten Spannungsverteilung mit Intensität $f_{C}$ gerechnet. Eine eventuell vorhandene Druckbewehrung in der Druckzone wird näherungsweise nicht berücksichtigt.

Wenn die Bügelbewehrung fliesst, kann die für die Aufnahme der Querkraft erforderliche Anzahl Bügel bestimmt und daraus die Druckfeldneigung ermittelt werden:

$$
\tan \vartheta=\frac{V}{A_{y} \cdot f} \operatorname{syy} \cdot \frac{s}{z}
$$

(Die Bügel werden als "verschmiert" betrachtet und der Hebelarm z wird unter der Voraussetzung, dass die Spannungsverteilung in der Druckzone im Bruchzustand konstant ist, bestimmt.)

Bei Balken mit kleiner Schubschlankheit kann diese Neigung kleiner sein als diejenige einer direkten Druckstrebe zwischen Lasteinleitungsplatte und Auflagerplatte (Bild A 1.2, Wert $\tan \vartheta_{\min }$ in der Tabelle A 1.4).

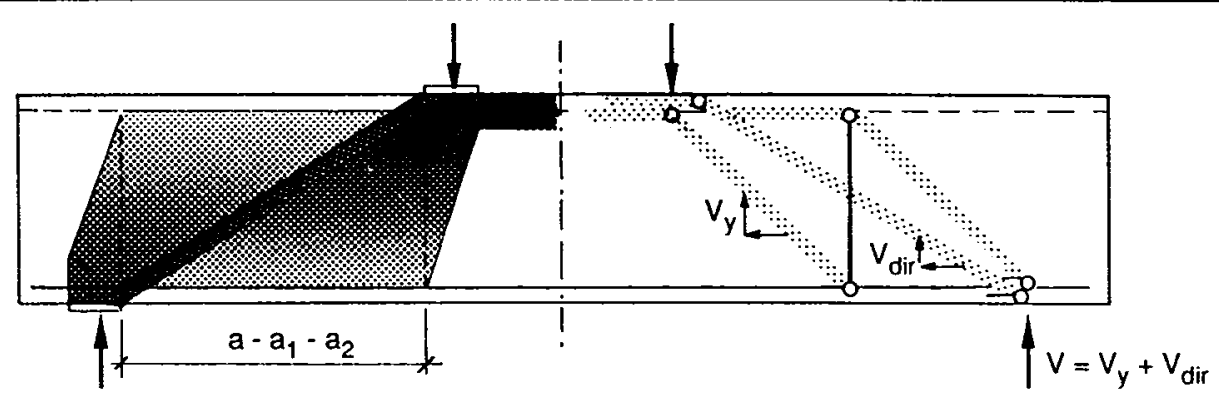

Bild A 1.2: Spannungsfeld eines Balkens mit teilweiser Direktabstützung 
Anders betrachtet ist in solchen Fällen die Fliesskraft sămtlicher Bügel zwischen den zwei Verteilplatten

$$
v_{y}=\frac{A_{y} \cdot f \text { syy }}{s} \cdot\left(a-a_{1}-a_{2}\right)
$$

kleiner als die experimentell ermittelte Bruchlast. Da die Betonzugfestigkeit nicht berücksichtigt wird, kann der fehlende Anteil Querkraft nur mit der direkten Druckstrebe aufgenommen werden:

$$
\mathrm{V}_{\mathrm{dir}}=\mathrm{V}-\mathrm{V}_{\mathrm{y}}
$$

Auch diese Versuche mit teilweiser Direktabstützung werden aussortiert (Tabelle A 1.4).

In der Tabelle A 1.5 sind die Resultate der Versuche im Regime BS gemăss Abschnitt 4.4 zusammengestelt. In diesem Regime bricht der Beton im Steg nachdem die Schubbewehrung geflossen ist.

Die effektive Betonfestigkeit (letzte Kolonne der Tabelle) wird mit folgender Gleichung ermittelt:

$$
-\sigma_{c} \quad=f_{c e}=\frac{V}{t \cdot z} \cdot \frac{1}{\sin \vartheta \cdot \cos \vartheta}
$$

Für das Regime B wird gemăss Plastizitătstheorie eine Druckstrebenneigung $\vartheta=45^{\circ}$ angenommen. In diesem Regime sind theoretisch beide Bewehrungen noch nicht geflossen. Tabelle A 1.6 zeigt die Resultate dieser Versuche.

Die in der letzten Kolonne dargestellte Querdehnung wird wie folgt näherungsweise berechnet:

$$
\begin{array}{ll}
\varepsilon_{1}=\varepsilon_{x}+\varepsilon_{y}-\varepsilon_{3} & \\
\varepsilon_{3}=-0.002 & \text { (geschătzte Längsstauchung des Betons) } \\
\varepsilon_{x}=\frac{V \cdot a}{z \cdot A_{x} \cdot E_{s}} & \text { (maximale Dehnung der Längsbewehrung) } \\
\varepsilon_{y}=\frac{V \cdot s}{z \cdot A_{y} \cdot E_{s}} & \text { (Dehnung der Schubbewehrung) }
\end{array}
$$

Es ist zu bemerken, dass die Dehnungen der Bewehrungen ohne Berücksichtung von "Tension Stiffening" berechnet worden sind. 
Nach den im Abschnitt 4.4 ermittelten kinematischen Relationen ist die Druckfeldneigung $\vartheta$ eine Funktion der Dehnungen $\varepsilon_{x}$ und $\varepsilon_{y}$ und der Betonstauchung $\varepsilon_{3}$, was hier nicht berücksichtigt wird. Ferner wird hier mit der maximalen Dehnung in Lăngsrichtung gerechnet (in Balkenmitte und auf der Balkenzugseite). Die so ermittelte Querdehnung ist somit nur als grobe Abschätzung der effektiv vorhandenen Dehnung zu betrachten.

Die Resultate dieser Auswertung sind in den Bildern 3.13b, 3.13c und 4.22c graphisch dargestellt.

Die Resultate weiterer Versuche mit sehr grossen Betonüberdeckungen $t_{c}\left(t_{c}>0.2 t\right)$, die in diesen Bildem nicht dargestellt sind, sind in den Tabellen $A 1.7$ und $A 1.8$ zusammengestellt. 


\begin{tabular}{|c|c|}
\hline 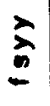 & 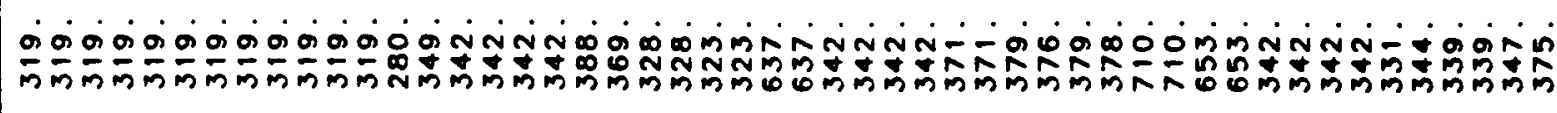 \\
\hline n & 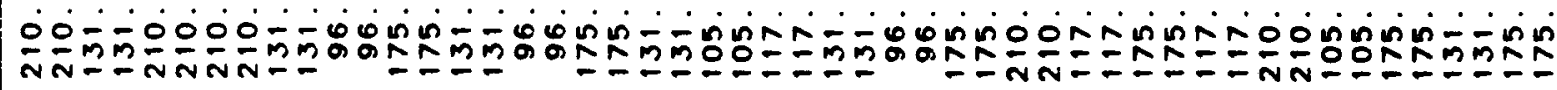 \\
\hline$\hat{z}$ & 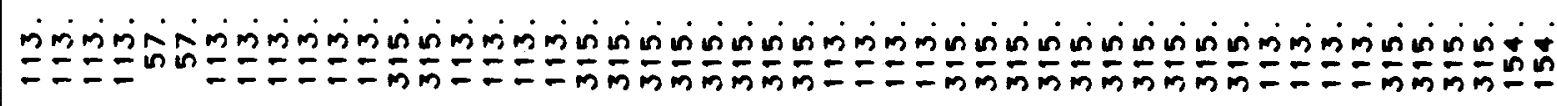 \\
\hline$\frac{x}{n}$ & 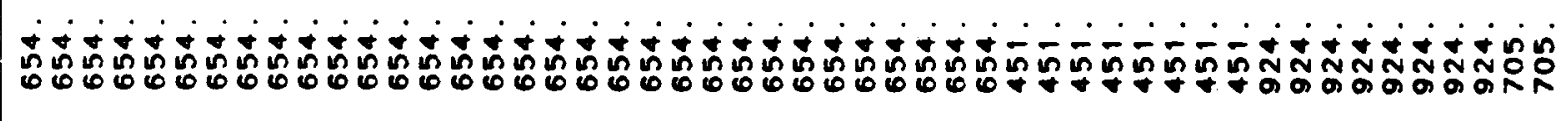 \\
\hline 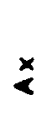 & 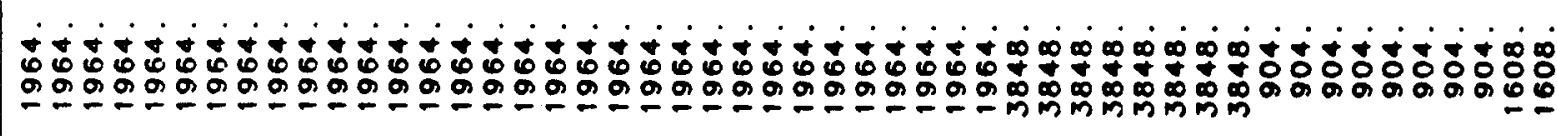 \\
\hline$\Xi$ & mar \\
\hline ฉ & หลำ \\
\hline$\overline{0}$ & หลำ \\
\hline$\stackrel{0}{2}$ & 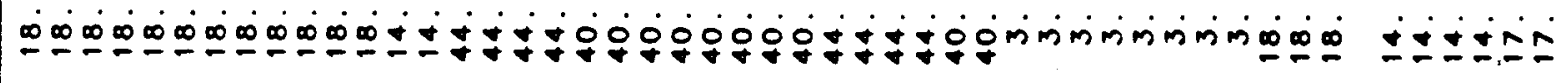 \\
\hline$\overline{0}$ & 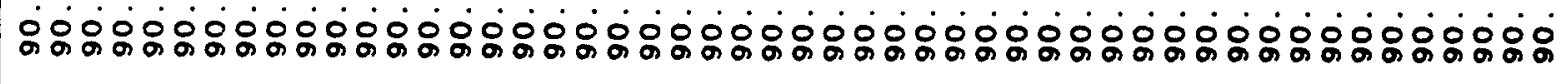 \\
\hline$=$ & ¿ం: \\
\hline - & 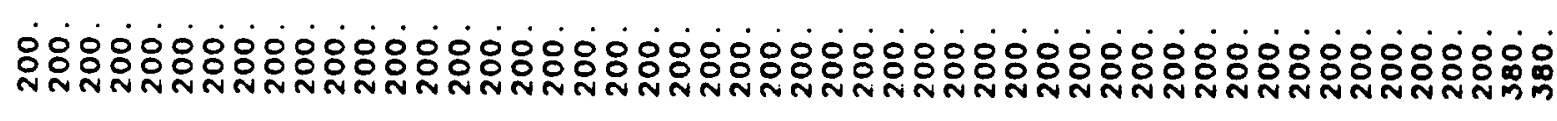 \\
\hline 0 & 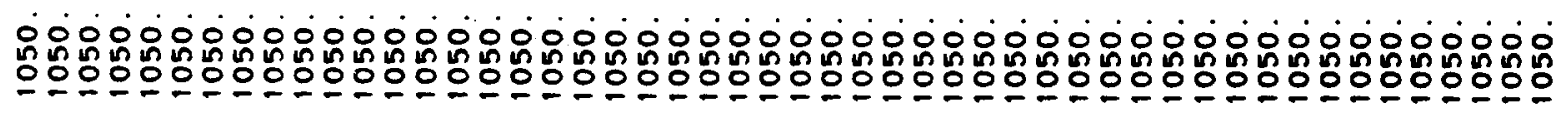 \\
\hline 0 & 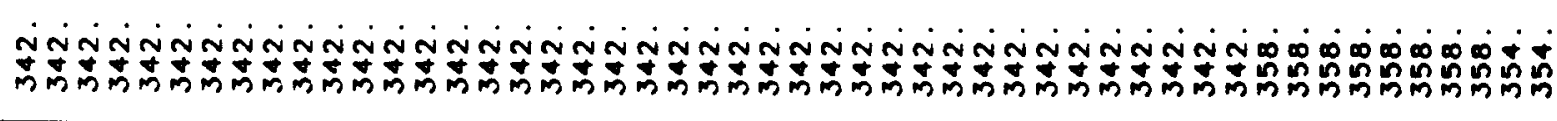 \\
\hline$>$ & 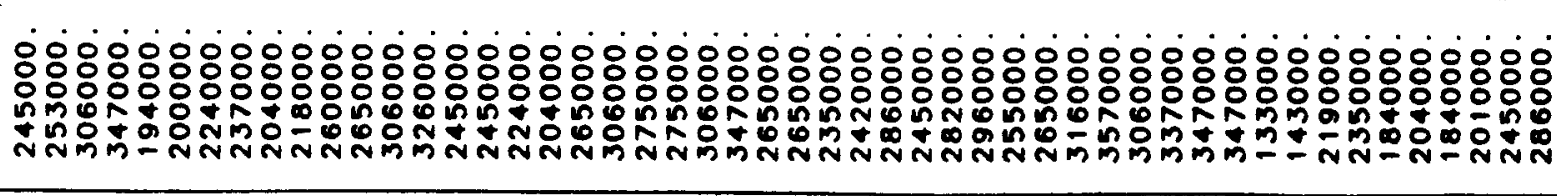 \\
\hline 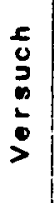 & 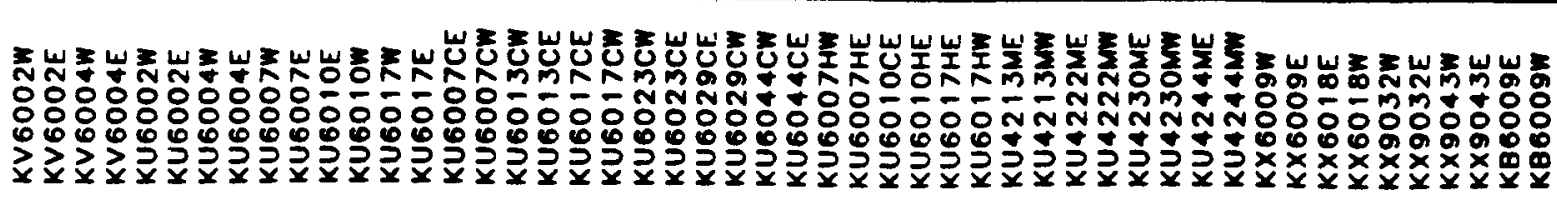 \\
\hline
\end{tabular}

Tabelle A 1.2: Versuchsdaten 


\begin{tabular}{|c|c|}
\hline 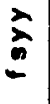 & 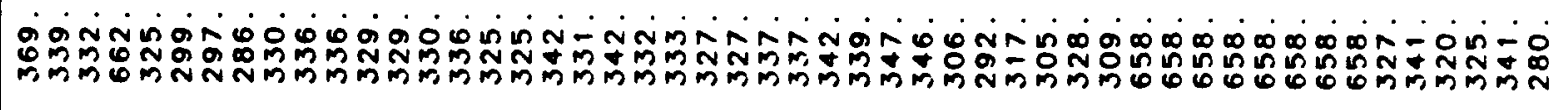 \\
\hline$\infty$ & 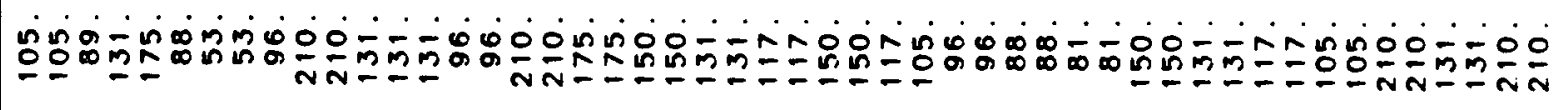 \\
\hline$\dot{z}$ & 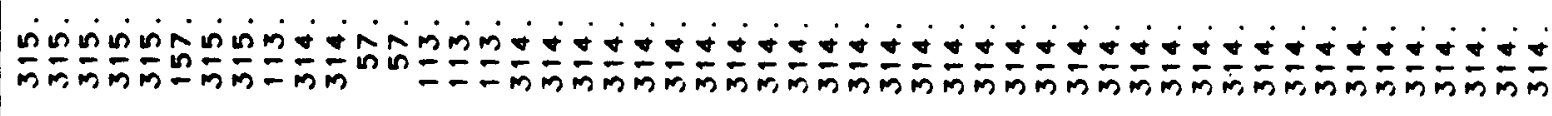 \\
\hline à & 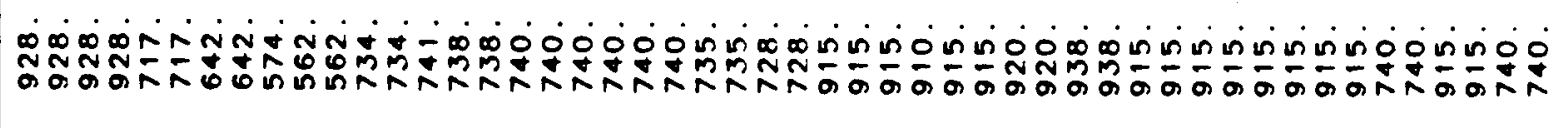 \\
\hline 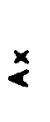 & : \\
\hline$\leq$ & 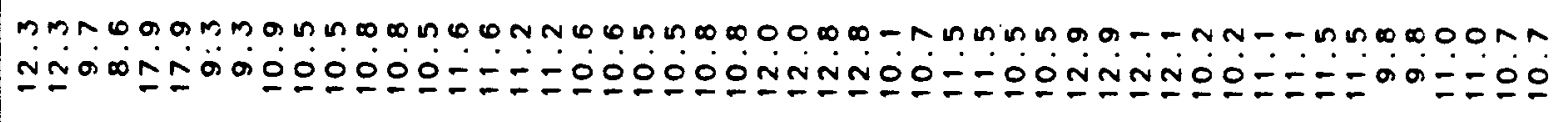 \\
\hline ס & 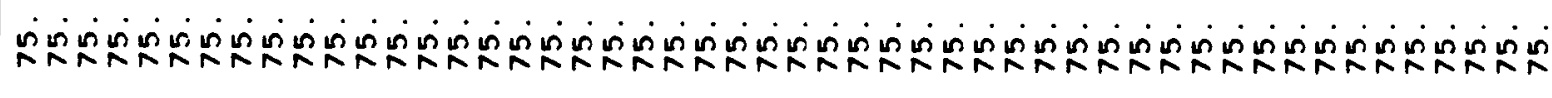 \\
\hline$\overline{0}$ & ํำ \\
\hline$\stackrel{0}{2}$ & 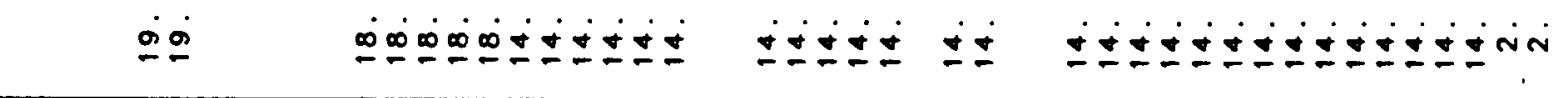 \\
\hline 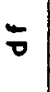 & 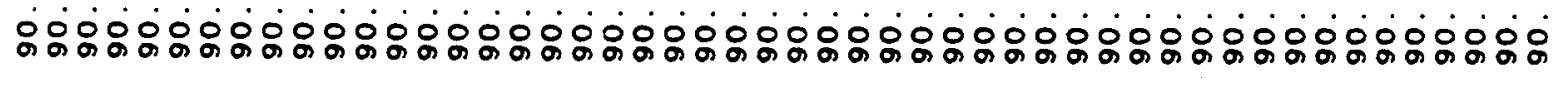 \\
\hline$=$ & : \\
\hline- & 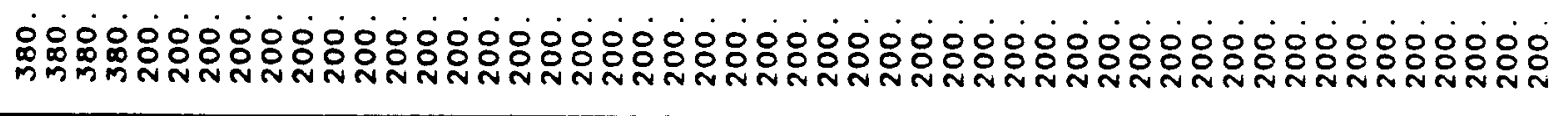 \\
\hline 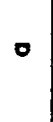 & 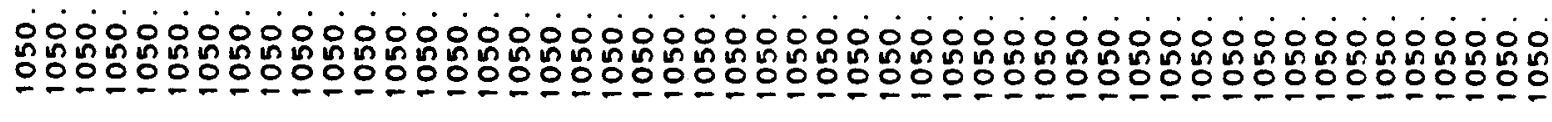 \\
\hline$\nabla$ & 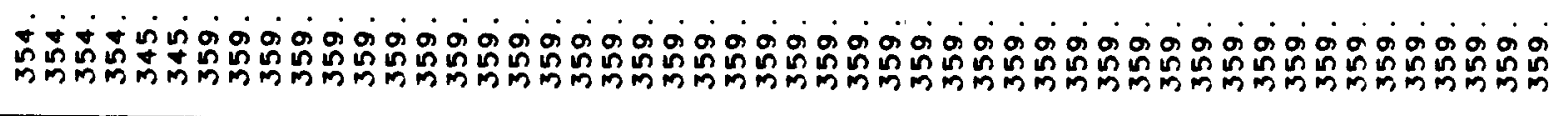 \\
\hline$>$ & 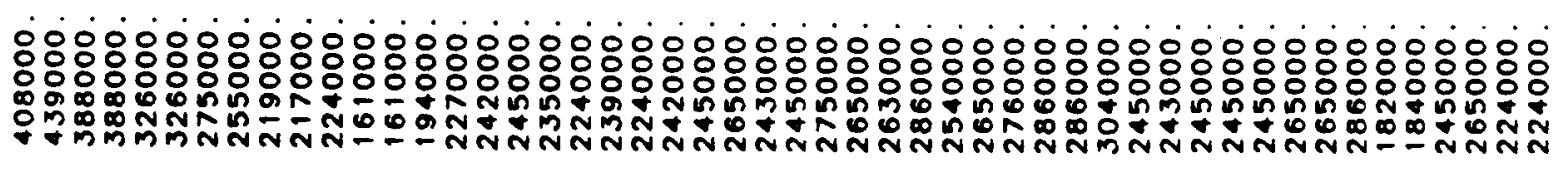 \\
\hline 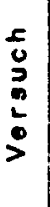 & 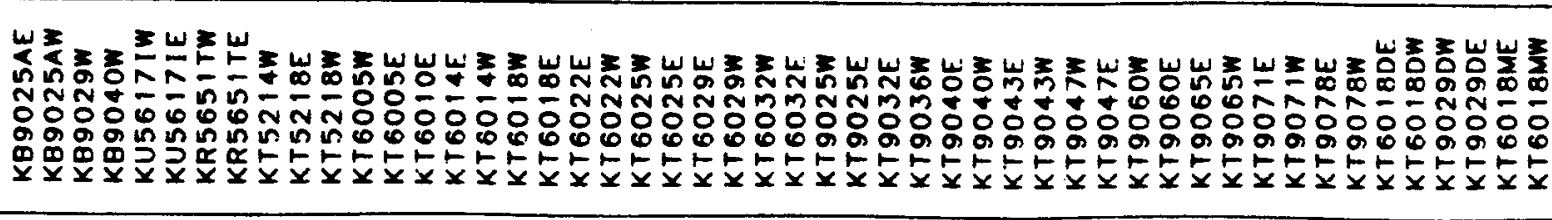 \\
\hline
\end{tabular}

Tabelle A 1.2: Versuchsdaten (Fortsetzung) 
$-145-$

\begin{tabular}{|c|c|}
\hline ì & 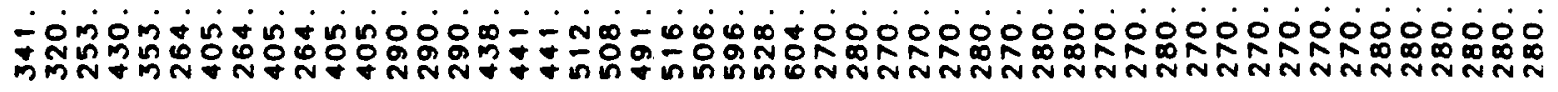 \\
\hline - & 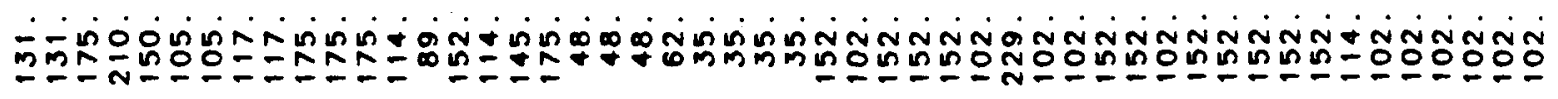 \\
\hline$\hat{k}$ & móñ \\
\hline$\stackrel{x}{x}$ & 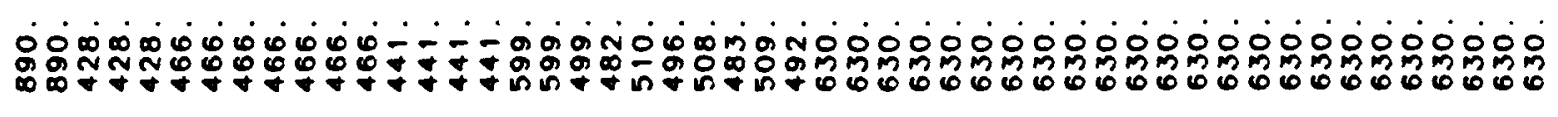 \\
\hline$\stackrel{x}{x}$ & 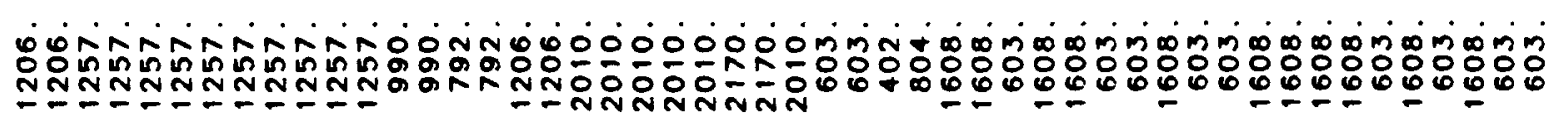 \\
\hline$\underline{-1}$ & 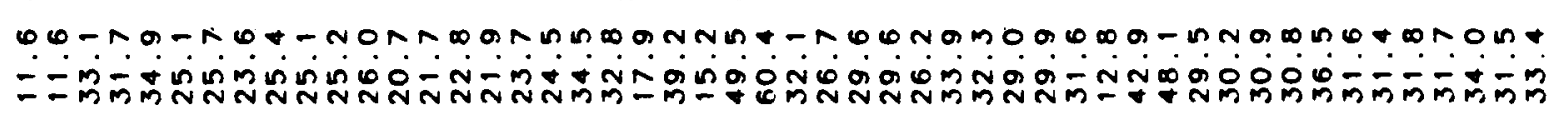 \\
\hline ช & 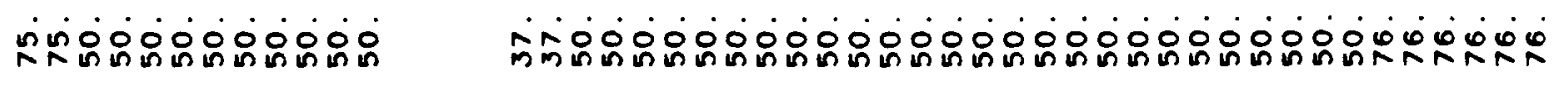 \\
\hline$\overline{0}$ & 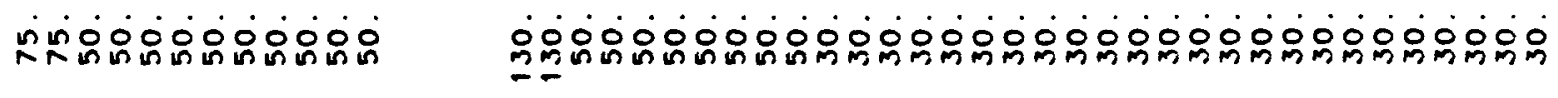 \\
\hline$\stackrel{0}{2}$ & 퓨류 \\
\hline 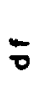 & ¿용요 \\
\hline$=$ & 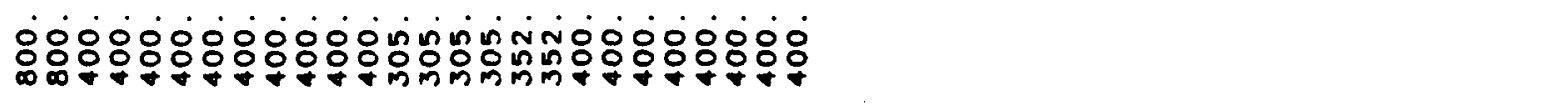 \\
\hline- & 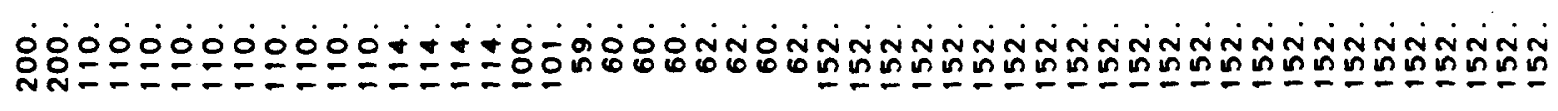 \\
\hline 0 & 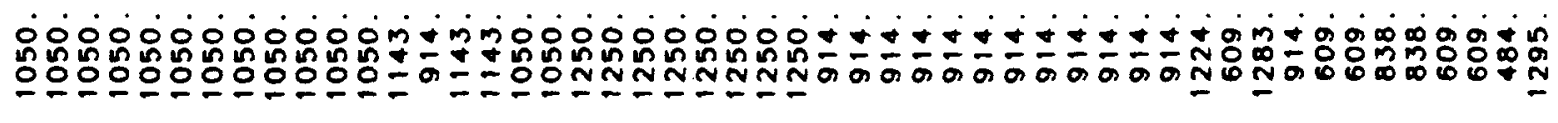 \\
\hline$\nabla$ & 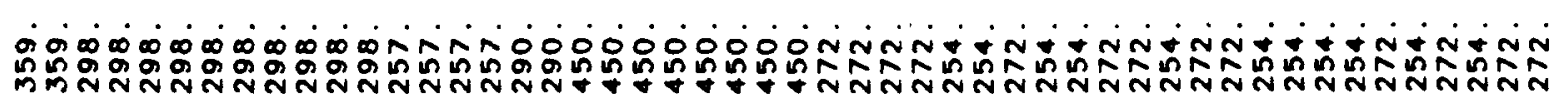 \\
\hline$>$ & 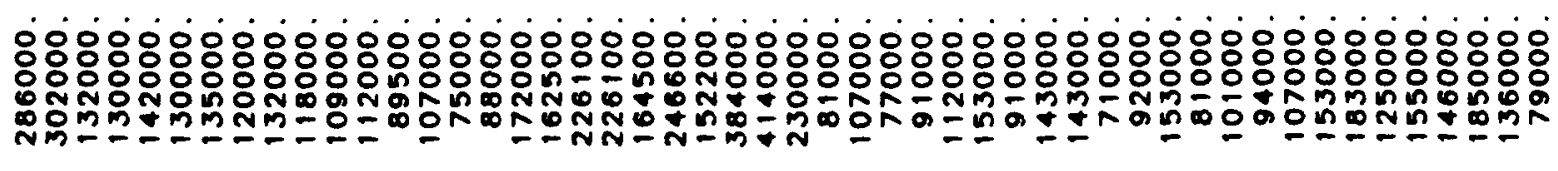 \\
\hline 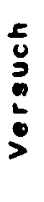 & 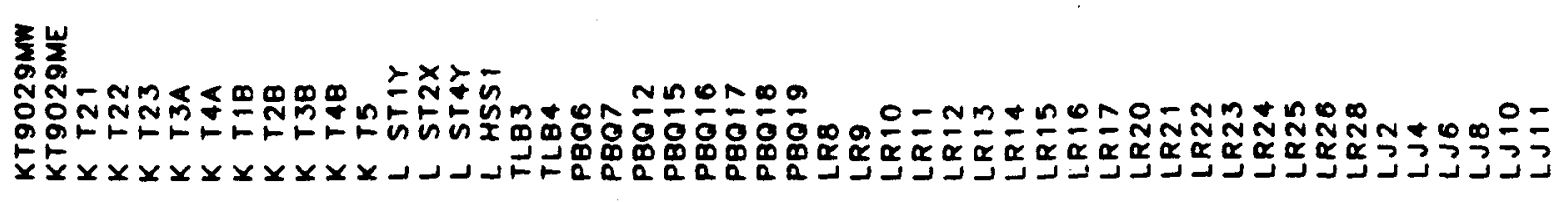 \\
\hline
\end{tabular}

Tabelle A 1.2: Versuchsdaten (Fortsetzung) 


\begin{tabular}{|c|c|}
\hline$\underline{a}$ & 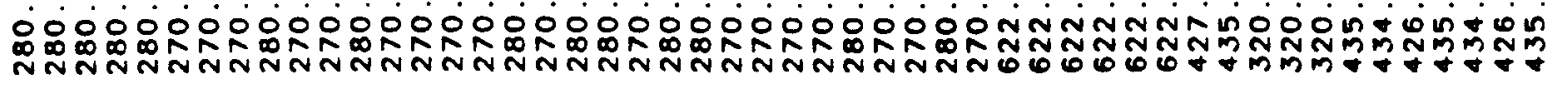 \\
\hline$n$ & 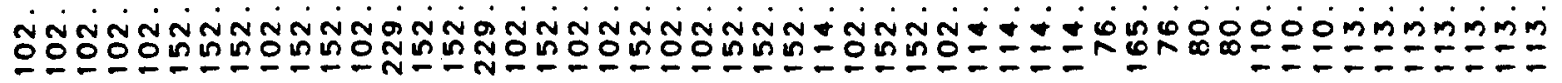 \\
\hline$\hat{\imath}$ & 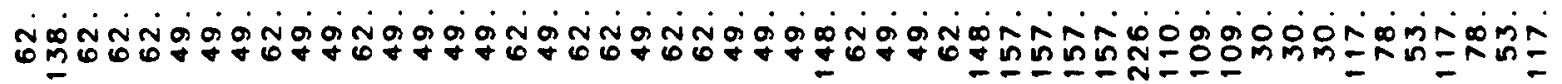 \\
\hline$\frac{x}{2}$ & 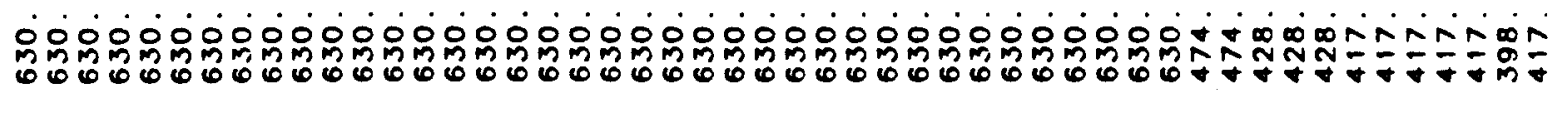 \\
\hline$\stackrel{x}{x}$ & 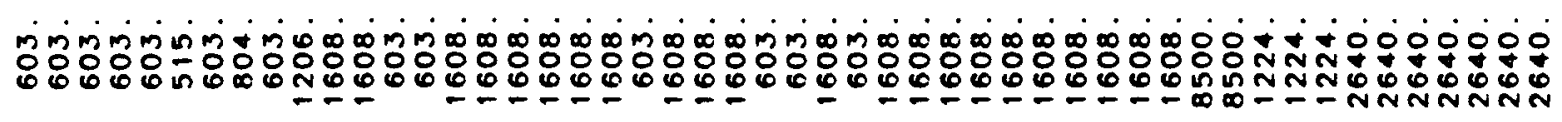 \\
\hline 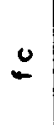 & 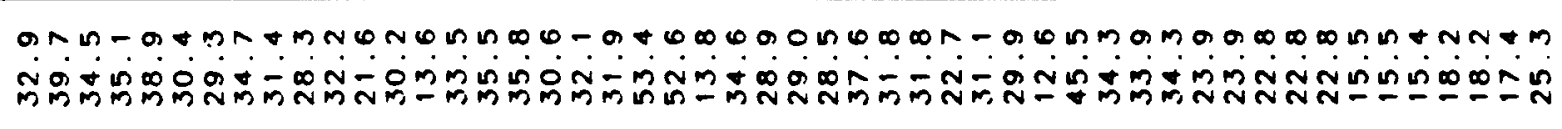 \\
\hline : & 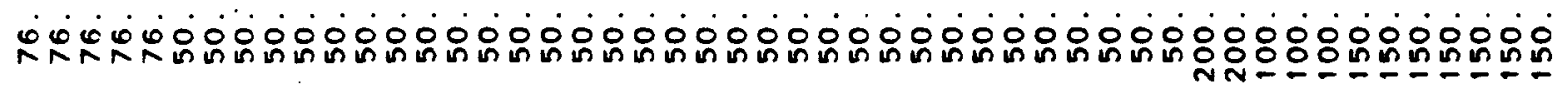 \\
\hline$\overline{0}$ & 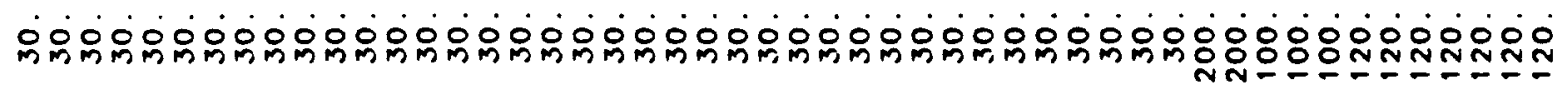 \\
\hline$\stackrel{2}{2}$ & 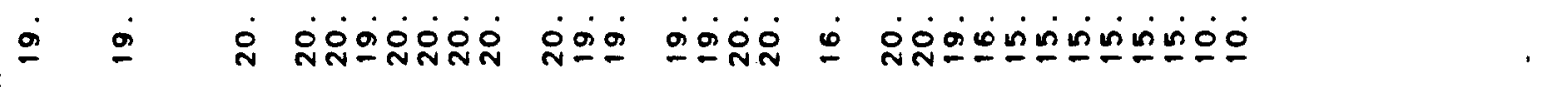 \\
\hline$\overline{0}$ & 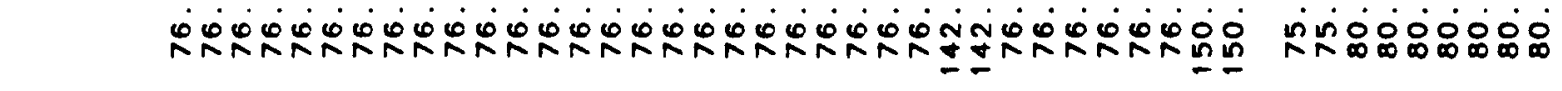 \\
\hline$=$ & 응 \\
\hline- & 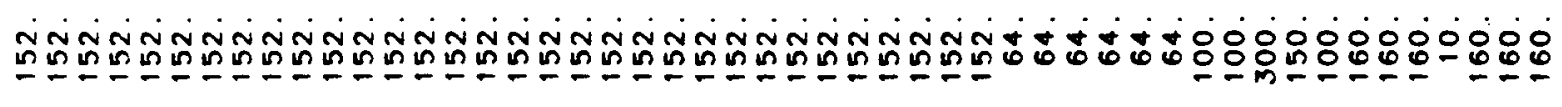 \\
\hline$\circ$ & 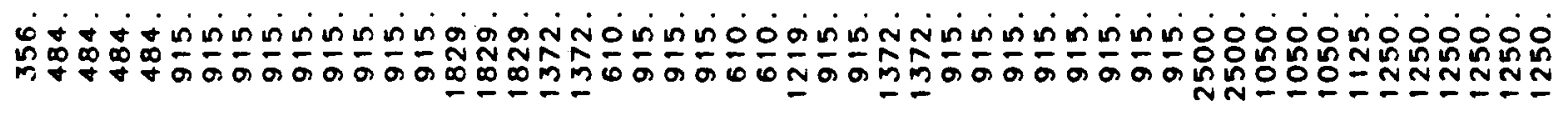 \\
\hline 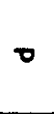 & 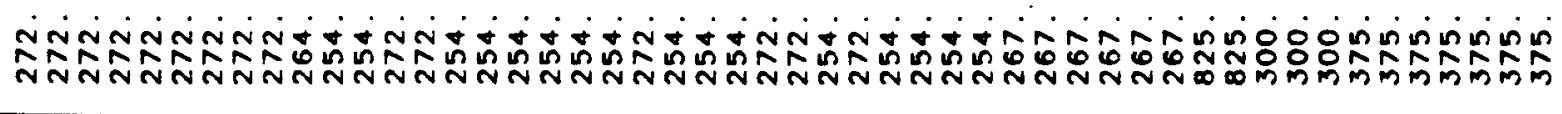 \\
\hline$>$ & $\begin{array}{l}80 \\
808 \\
0 \\
\circ\end{array}$ \\
\hline $\begin{array}{l}5 \\
\vdots \\
0 \\
\vdots \\
\vdots \\
\end{array}$ & 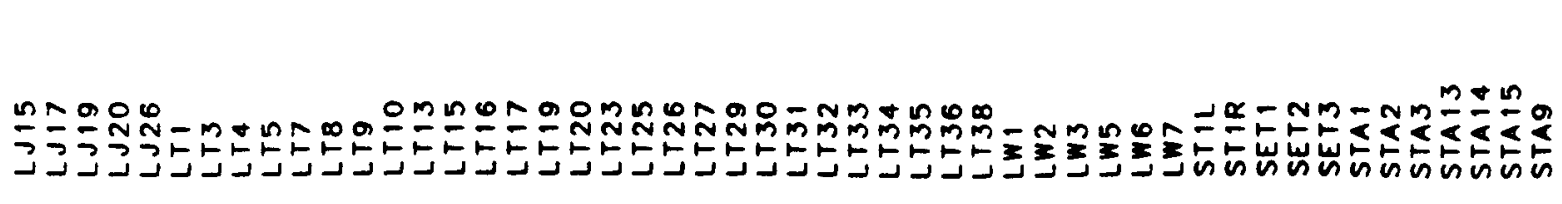 \\
\hline
\end{tabular}

Tabelle A 1.2: Versuchsdaten (Fortsetzung) 


\begin{tabular}{|c|c|}
\hline 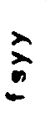 & 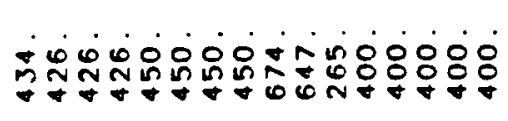 \\
\hline 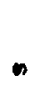 & 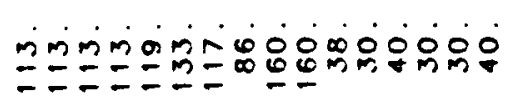 \\
\hline$\hat{\imath}$ & 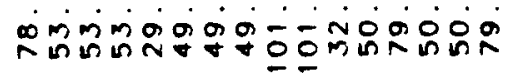 \\
\hline$i^{x}$ & 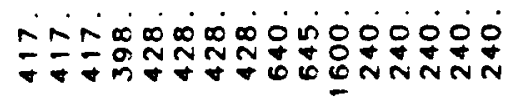 \\
\hline$\stackrel{x}{x}$ & 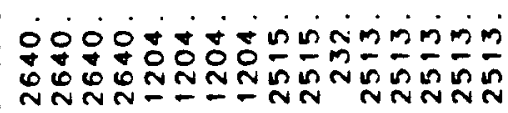 \\
\hline 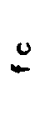 & 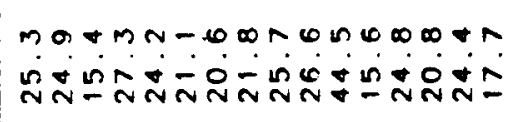 \\
\hline 乏 & 品品品: \\
\hline$\overline{0}$ & 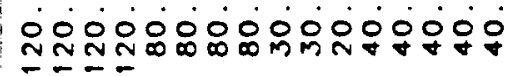 \\
\hline 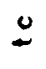 & 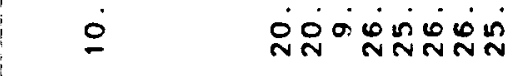 \\
\hline$\overline{7}$ & ه̊: \\
\hline$=$ & 영요 \\
\hline ــ & $\therefore 8: 000000$ \\
\hline ○ & 品on \\
\hline 0 & 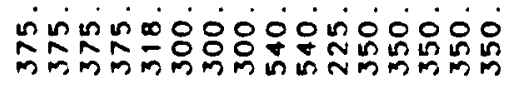 \\
\hline$>$ & 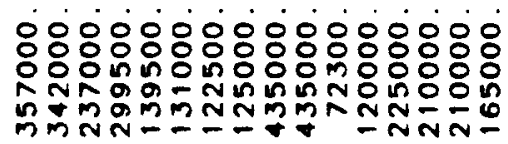 \\
\hline $\begin{array}{l}5 \\
0 \\
3 \\
0 \\
\text { s } \\
>\end{array}$ & 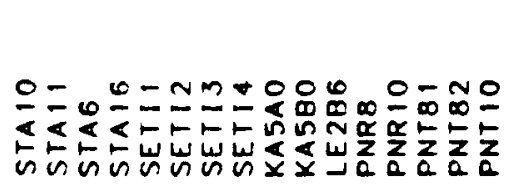 \\
\hline
\end{tabular}

Tabelle A 1.2: Versuchsdaten (Fortsetzung) 


\begin{tabular}{|c|c|c|c|}
\hline versuch & $v$ & Vflex & $v / v f l e x$ \\
\hline 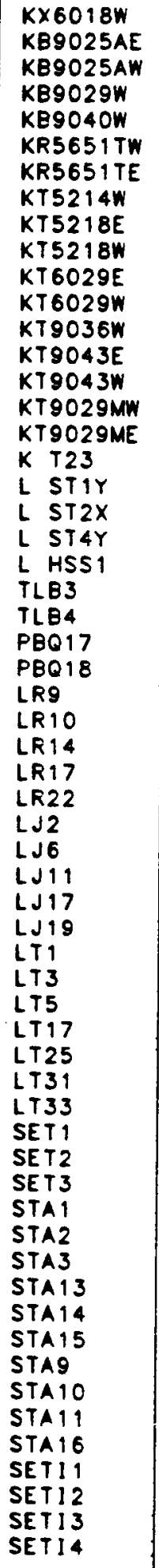 & $\begin{array}{l}235000 \\
408000 \\
439000 \\
388000 \\
388000 \\
275000 \\
255000 \\
219000 \\
217000 \\
224000 \\
245000 \\
265000 \\
286000 \\
276000 \\
286000 \\
286000 \\
302000 \\
142000 \\
89500 \\
107000 \\
75000 \\
88000 \\
172000 \\
162500 \\
384000 \\
414000 \\
107000 \\
77000 \\
91000 \\
71000 \\
81000 \\
125000 \\
146000 \\
79000 \\
265000 \\
183000 \\
110000 \\
105000 \\
139000 \\
134000 \\
114000 \\
95000 \\
105000 \\
140000 \\
131500 \\
127500 \\
347500 \\
331000 \\
283000 \\
350000 \\
340000 \\
298000 \\
350000 \\
357000 \\
342000 \\
299500 \\
139500 \\
131000 \\
122500 \\
125000 \\
.\end{array}$ & 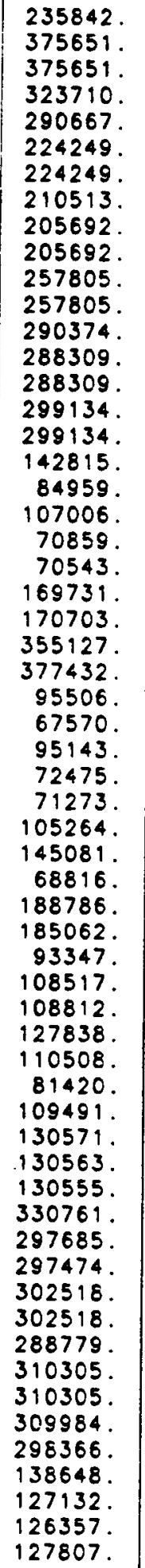 & $\begin{array}{l}0.996 \\
1.086 \\
1.169 \\
1.199 \\
1.335 \\
1.226 \\
1.137 \\
1.040 \\
1.055 \\
1.089 \\
1.09\end{array}$ \\
\hline
\end{tabular}

Tabelle A 1.3: Versuche mit Fliessen der Långsbewehrung 


\begin{tabular}{|c|c|c|c|c|}
\hline Versueh & $v$ & & $v_{y}$ & voir $/ V$ \\
\hline 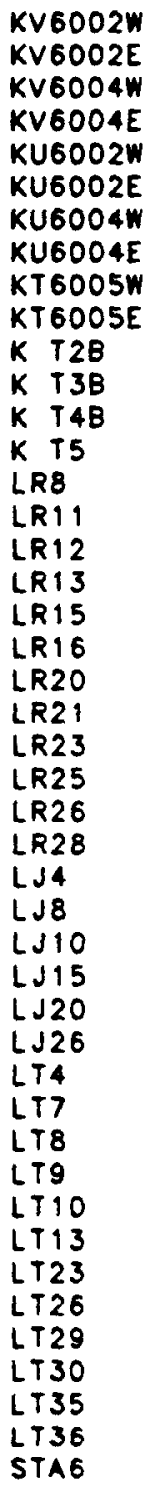 & $\begin{array}{r}245000 . \\
253000 . \\
306000 . \\
347000 . \\
194000 . \\
200000 . \\
224000 . \\
237000 . \\
161000 . \\
161000 . \\
132000 . \\
118000 . \\
109000 . \\
112000 . \\
81000 . \\
91000 . \\
112000 . \\
153000 . \\
143000 . \\
143000 . \\
92000 . \\
153000 . \\
101000 . \\
107000 . \\
153000 . \\
183000 . \\
155000 . \\
185000 . \\
136000 . \\
206000 . \\
160000 . \\
175000 . \\
110000 . \\
109000 . \\
124000 . \\
154000 . \\
87000 . \\
90000 . \\
194000 . \\
179000 . \\
152000 . \\
109000 . \\
115000 . \\
179000 . \\
237000 .\end{array}$ & $\begin{array}{l}0.365 \\
0.364 \\
0.361 \\
0.358 \\
0.357 \\
0.357 \\
0.356 \\
0.354 \\
0.366 \\
0.366 \\
0.287 \\
0.290 \\
0.292 \\
0.292 \\
0.279 \\
0.271 \\
0.247 \\
0.204 \\
0.202 \\
0.213 \\
0.295 \\
0.249 \\
0.462 \\
0.243 \\
0.406 \\
0.367 \\
0.244 \\
0.396 \\
0.645 \\
0.967 \\
0.640 \\
0.641 \\
0.315 \\
0.302 \\
0.290 \\
0.276 \\
0.316 \\
0.302 \\
0.455 \\
0.292 \\
0.462 \\
0.500 \\
0.189 \\
0.273 \\
0.353\end{array}$ & $\begin{array}{r}154487 . \\
154487 . \\
247651 . \\
247651 . \\
77927 . \\
77927 . \\
154487 . \\
154487 . \\
128837 . \\
128837 . \\
128250 . \\
81689 . \\
85744 . \\
85744 . \\
72591 . \\
72591 . \\
72591 . \\
141944 . \\
141944 . \\
141944 . \\
72591 . \\
141944 . \\
46044 . \\
72591 . \\
46044 . \\
177911 . \\
124584 . \\
85609 . \\
64334 . \\
42549 . \\
64334 . \\
64334 . \\
72678 . \\
72678 . \\
72678 . \\
142114 . \\
48240 . \\
72678 . \\
90204 . \\
142114 . \\
46131 . \\
46131 . \\
112455 . \\
142114 . \\
195809 .\end{array}$ & $\begin{array}{l}0.369 \\
0.389 \\
0.191 \\
0.286 \\
0.598 \\
0.610 \\
0.310 \\
0.348 \\
0.200 \\
0.200 \\
0.028 \\
0.308 \\
0.213 \\
0.234 \\
0.104 \\
0.202 \\
0.352 \\
0.072 \\
0.007 \\
0.007 \\
0.211 \\
0.072 \\
0.544 \\
0.322 \\
0.699 \\
0.028 \\
0.196 \\
0.537 \\
0.527 \\
0.793 \\
0.598 \\
0.632 \\
0.339 \\
0.333 \\
0.414 \\
0.077 \\
0.446 \\
0.192 \\
0.535 \\
0.206 \\
0.697 \\
0.577 \\
0.022 \\
0.206 \\
0.174\end{array}$ \\
\hline
\end{tabular}

Tabelle A 1.4: Versuche mit teilweiser Direktabstützung 


\begin{tabular}{|c|c|c|c|c|c|c|}
\hline versuch & $x / 0$ & $2 / 0$ & & & & $f c e / f c$ \\
\hline 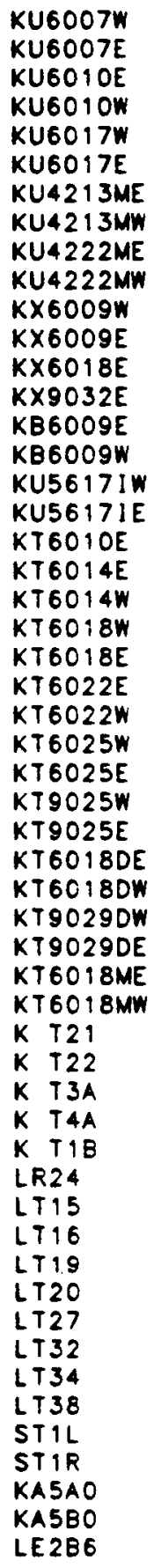 & $\begin{array}{l}0.170 \\
0.183 \\
0.196 \\
0.200 \\
0.189 \\
0.203 \\
0.227 \\
0.238 \\
0.258 \\
0.414 \\
0.208 \\
0.226 \\
0.360 \\
0.406 \\
0.307 \\
0.442 \\
0.226 \\
0.226 \\
0.210 \\
0.224 \\
0.242 \\
0.270 \\
0.243 \\
0.245 \\
0.308 \\
0.248 \\
0.338 \\
0.250 \\
0.240 \\
0.211 \\
0.214 \\
0.292 \\
0.406 \\
0.243 \\
0.243 \\
0.126 \\
0.130 \\
0.167 \\
0.170 \\
0.164 \\
0.548 \\
0.157 \\
0.124 \\
0.129 \\
0.163 \\
0.255 \\
0.195 \\
0.287 \\
0.197 \\
0.086 \\
0.086 \\
0.133 \\
0.128 \\
0.099\end{array}$ & $\begin{array}{l}0.915 \\
0.908 \\
0.902 \\
0.900 \\
0.906 \\
0.899 \\
0.886 \\
0.881 \\
0.871 \\
0.842 \\
0.896 \\
0.887 \\
0.857 \\
0.847 \\
0.859 \\
0.816 \\
0.887 \\
0.887 \\
0.895 \\
0.888 \\
0.879 \\
0.872 \\
0.878 \\
0.877 \\
0.866 \\
0.876 \\
0.861 \\
0.875 \\
0.880 \\
0.894 \\
0.893 \\
0.869 \\
0.847 \\
0.879 \\
0.879 \\
0.937 \\
0.935 \\
0.916 \\
0.915 \\
0.918 \\
0.726 \\
0.922 \\
0.938 \\
0.935 \\
0.918 \\
0.873 \\
0.902 \\
0.857 \\
0.901 \\
0.957 \\
0.957 \\
0.933 \\
0.936 \\
0.950\end{array}$ & $\begin{array}{l}0.094 \\
0.094 \\
0.114 \\
0.114 \\
0.125 \\
0.156 \\
0.200 \\
0.199 \\
0.323 \\
0.322 \\
0.126 \\
0.126 \\
0.196 \\
0.364 \\
0.075 \\
0.081 \\
0.163 \\
0.149 \\
0.136 \\
0.170 \\
0.165 \\
0.217 \\
0.228 \\
0.280 \\
0.289 \\
0.331 \\
0.332 \\
0.280 \\
0.277 \\
0.249 \\
0.260 \\
0.349 \\
0.354 \\
0.238 \\
0.196 \\
0.040 \\
0.045 \\
0.052 \\
0.053 \\
0.050 \\
0.019 \\
0.017 \\
0.011 \\
0.019 \\
0.035 \\
0.081 \\
0.081 \\
0.018 \\
0.074 \\
0.243 \\
0.248 \\
0.138 \\
0.128 \\
0.201\end{array}$ & $\begin{array}{l}0.222 \\
0.239 \\
0.255 \\
0.261 \\
0.246 \\
0.264 \\
0.296 \\
0.310 \\
0.336 \\
0.392 \\
0.284 \\
0.308 \\
0.330 \\
0.396 \\
0.198 \\
0.244 \\
0.298 \\
0.298 \\
0.288 \\
0.307 \\
0.330 \\
0.349 \\
0.333 \\
0.335 \\
0.362 \\
0.339 \\
0.373 \\
0.342\end{array}$ & $\begin{array}{l}0.422 \\
0.392 \\
0.446 \\
0.436 \\
0.510 \\
0.592 \\
0.676 \\
0.641 \\
0.962 \\
0.821 \\
0.444 \\
0.409 \\
0.516 \\
0.921 \\
0.379 \\
0.333 \\
0.549 \\
0.501 \\
0.471 \\
0.555 \\
0.499 \\
0.621 \\
0.686 \\
0.835 \\
0.799 \\
0.876 \\
0.890 \\
0.818 \\
0.846 \\
0.862 \\
0.888 \\
0.977 \\
0.894 \\
0.718 \\
0.590 \\
0.309 \\
0.338 \\
0.301 \\
0.304 \\
0.293 \\
0.171 \\
0.196 \\
0.155 \\
0.195 \\
0.288 \\
0.286 \\
0.372 \\
0.169 \\
0.337 \\
0.574 \\
0.585 \\
0.493 \\
0.474 \\
0.660\end{array}$ & $\begin{array}{l}0.619 \\
0.702 \\
0.687 \\
0.712 \\
0.607 \\
0.602 \\
0.639 \\
0.681 \\
0.672 \\
0.800 \\
0.766 \\
0.881 \\
0.032 \\
0.794 \\
0.598 \\
0.812 \\
0.706 \\
0.744 \\
0.746 \\
0.723 \\
0.827 \\
0.779 \\
0.713 \\
0.682\end{array}$ \\
\hline
\end{tabular}

Tabelle A 1.5: Versuche mit Fliessen der Bügelbewehrung 


\begin{tabular}{|c|c|c|c|c|c|c|}
\hline Versuch & $x / 0$ & $2 / 0$ & & & $f c / f c$ & \\
\hline 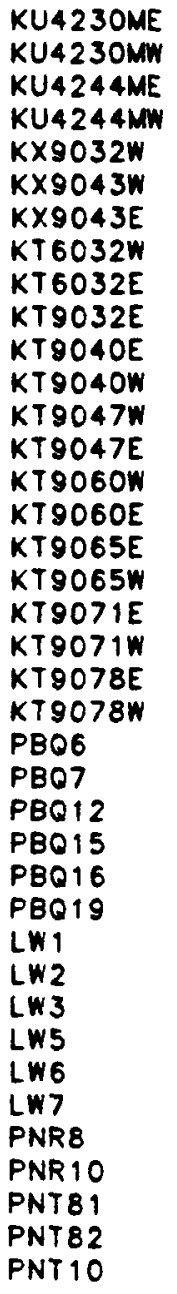 & $\begin{array}{l}0.231 \\
0.259 \\
0.281 \\
0.281 \\
0.262 \\
0.307 \\
0.443 \\
0.233 \\
0.236 \\
0.559 \\
0.282 \\
0.338 \\
0.286 \\
0.371 \\
0.233 \\
0.231 \\
0.401 \\
0.401 \\
0.281 \\
0.391 \\
0.338 \\
0.462 \\
0.107 \\
0.113 \\
0.154 \\
0.102 \\
0.169 \\
0.1177 \\
0.120 \\
0.164 \\
0.097 \\
0.124 \\
0.103 \\
0.105 \\
0.092 \\
0.110 \\
0.123 \\
0.104 \\
0.113\end{array}$ & $\begin{array}{l}0.884 \\
0.871 \\
0.866 \\
0.866 \\
0.873 \\
0.866 \\
0.839 \\
0.883 \\
0.882 \\
0.809 \\
0.870 \\
0.861 \\
0.870 \\
0.855 \\
0.883 \\
0.884 \\
0.849 \\
0.849 \\
0.871 \\
0.851 \\
0.861 \\
0.834 \\
0.947 \\
0.944 \\
0.923 \\
0.949 \\
0.916 \\
0.941 \\
0.940 \\
0.918 \\
0.952 \\
0.938 \\
0.948 \\
0.948 \\
0.954 \\
0.945 \\
0.939 \\
0.948 \\
0.944\end{array}$ & $\begin{array}{l}0.380 \\
0.380 \\
0.523 \\
0.523 \\
0.350 \\
0.497 \\
0.497 \\
0.377 \\
0.377 \\
0.461 \\
0.435 \\
0.415 \\
0.493 \\
0.464 \\
0.569 \\
0.569 \\
0.773 \\
0.773 \\
0.795 \\
0.795 \\
0.856 \\
0.856 \\
0.414 \\
0.425 \\
0.752 \\
0.357 \\
0.874 \\
0.494 \\
0.448 \\
1.062 \\
0.294 \\
0.585 \\
0.393 \\
0.410 \\
0.712 \\
0.531 \\
0.534 \\
0.455 \\
0.744\end{array}$ & $\begin{array}{l}0.301 \\
0.337 \\
0.349 \\
0.349 \\
0.346 \\
0.362 \\
0.408 \\
0.319 \\
0.322 \\
0.448 \\
0.353 \\
0.373 \\
0.355 \\
0.384 \\
0.319 \\
0.316 \\
0.394 \\
0.394 \\
0.353 \\
0.391 \\
0.373 \\
0.415 \\
0.261 \\
0.271 \\
0.369 \\
0.246 \\
0.392 \\
0.273 \\
0.333 \\
0.455 \\
0.269 \\
0.346 \\
0.288 \\
0.292 \\
0.384 \\
0.457 \\
0.5112 \\
0.432 \\
0.470\end{array}$ & $\begin{array}{l}0.602 \\
0.674 \\
0.697 \\
0.697 \\
0.693 \\
0.724 \\
0.816 \\
0.639 \\
0.645 \\
0.897 \\
0.707 \\
0.745 \\
0.710 \\
0.768 \\
0.639 \\
0.633 \\
0.789 \\
0.789 \\
0.705 \\
0.782 \\
0.745 \\
0.830 \\
0.522 \\
0.541 \\
0.737 \\
0.491 \\
0.784 \\
0.546 \\
0.666 \\
0.911 \\
0.538 \\
0.691 \\
0.575 \\
0.583 \\
0.768 \\
0.814 \\
1.025 \\
0.865 \\
0.941\end{array}$ & $\begin{array}{l}.00619 \\
.00669 \\
.00577 \\
.00577 \\
.00705 \\
.00668 \\
.00728 \\
.00676 \\
.00681 \\
.00763 \\
.00678 \\
.00704 \\
.00717 \\
.00759 \\
.00721 \\
.00716 \\
.00718 \\
.00718 \\
.00687 \\
.00739 \\
.00716 \\
.00775 \\
.00526 \\
.00527 \\
.00443 \\
.00557 \\
.00428 \\
.00536 \\
.00613 \\
.00438 \\
.00707 \\
.00600 \\
.00605 \\
.00603 \\
.00358 \\
.00467 \\
.00481 \\
.00478 \\
.00396\end{array}$ \\
\hline
\end{tabular}

Tabelle A 1.6: Versuche ohne Fliessen der Bügelbewehrung 
-152 -

\begin{tabular}{|l|c|c|c|c|c|c|}
\hline VersuCh & $x / d$ & $z / d$ & & & & fce/fc \\
\hline KU6007CE & 0.164 & 0.918 & 0.081 & 0.213 & 0.378 & 0.644 \\
KU6007CW & 0.164 & 0.918 & 0.081 & 0.213 & 0.378 & 0.644 \\
KU6013CW & 0.229 & 0.886 & 0.162 & 0.298 & 0.544 & 0.710 \\
KU6013CE & 0.206 & 0.897 & 0.162 & 0.268 & 0.605 & 0.605 \\
KU6017CE & 0.178 & 0.911 & 0.191 & 0.232 & 0.821 & 0.474 \\
KU6017CW & 0.210 & 0.895 & 0.181 & 0.273 & 0.665 & 0.592 \\
KU6023CW & 0.199 & 0.800 & 0.229 & 0.260 & 0.883 & 0.523 \\
KU6023CE & 0.199 & 0.900 & 0.229 & 0.260 & 0.883 & 0.523 \\
KU6029CE & 0.190 & 0.905 & 0.242 & 0.247 & 0.980 & 0.494 \\
KU6029CW & 0.219 & 0.891 & 0.242 & 0.285 & 0.851 & 0.577 \\
KU6007HW & 0.188 & 0.906 & 0.095 & 0.245 & 0.389 & 0.724 \\
KU6007HE & 0.194 & 0.903 & 0.095 & 0.253 & 0.376 & 0.767 \\
KU6010CE & 0.183 & 0.908 & 0.104 & 0.238 & 0.437 & 0.650 \\
KU6010HE & 0.168 & 0.916 & 0.112 & 0.218 & 0.515 & 0.537 \\
KU6017HE & 0.170 & 0.915 & 0.164 & 0.222 & 0.741 & 0.464 \\
KU6017HW & 0.180 & 0.910 & 0.164 & 0.234 & 0.702 & 0.498 \\
\hline
\end{tabular}

Tabelle A 1.7: Versuche mit Fliessen der Bügelbewehrung $\left(t_{c}>0.2 t\right)$

\begin{tabular}{|l|c|c|c|c|c|c|}
\hline Versuch & $x / d$ & $z / d$ & & & $f c e / f c$ & \\
\hline KU6044CW & 0.223 & 0.888 & 0.572 & 0.291 & 0.581 & .00595 \\
KU6044CE & 0.223 & 0.888 & 0.572 & 0.291 & 0.581 & .00595 \\
\hline
\end{tabular}

Tabelle A 1.8: Versuche ohne Fliessen der Bügelbewehrung $\left(t_{c}>0.2 t\right)$ 
Anhang A2: Auswertung der Versuche von Richart, Brandzaeg, Brown (1929) an zylindrischen Stützenelementen mit Umschnürungsbewehrung

Die Probekőper waren $1016 \mathrm{~mm}$ lang und hatten einen Durchmesser von $254 \mathrm{~mm}$. Die Querbewehrung war spiralförmig und hatte praktisch keine Betonüberdeckung. Die Versuchsdaten und die Resultate sind in der Tabelle A 2 zusammengestellt.

\begin{tabular}{|c|c|c|c|c|c|c|c|c|c|}
\hline Versuch & d & 8 & Ast & & fe & $1=y$ & & $9 / d$ & fes \\
\hline $\begin{array}{l}11 \\
12 \\
13 \\
21 \\
22 \\
23 \\
31 \\
32 \\
33 \\
41 \\
42 \\
43 \\
51 \\
52 \\
53\end{array}$ & $\begin{array}{l}254 . \\
254 . \\
254 . \\
254 . \\
254 . \\
254 . \\
254 . \\
254 . \\
254 . \\
254 . \\
254 . \\
254 . \\
254 . \\
254 . \\
254 .\end{array}$ & $\begin{array}{l}25 . \\
25 . \\
25 . \\
25 . \\
25 . \\
25 . \\
25 . \\
25 . \\
25 . \\
24 . \\
24 . \\
24 . \\
26 . \\
26 . \\
26 .\end{array}$ & $\begin{array}{l}8 . \\
8 . \\
8 . \\
18 . \\
18 . \\
18 . \\
33 . \\
33 . \\
33 . \\
40 . \\
40 . \\
40 . \\
72 . \\
72 . \\
72 .\end{array}$ & $\begin{array}{l}0.0025 \\
0.0025 \\
0.0025 \\
0.0055 \\
0.0055 \\
0.0055 \\
0.0102 \\
0.0102 \\
0.0102 \\
0.0130 \\
0.0130 \\
0.0130 \\
0.0220 \\
0.0220 \\
0.0220\end{array}$ & $\begin{array}{l}16.2 \\
16.0 \\
16.0 \\
16.0 \\
17.4 \\
16.5 \\
17.5 \\
15.3 \\
16.9 \\
16.5 \\
16.0 \\
15.0 \\
16.6 \\
17.5 \\
16.3\end{array}$ & $\begin{array}{l}462 . \\
462 . \\
462 . \\
421 . \\
421 . \\
421 . \\
262 . \\
262 . \\
262 . \\
276 . \\
276 . \\
276 . \\
317 . \\
317 . \\
317 .\end{array}$ & $\begin{array}{l}0.0702 \\
0.0672 \\
0.0713 \\
0.1456 \\
0.1344 \\
0.1413 \\
0.1534 \\
0.1753 \\
0.1589 \\
0.2173 \\
0.2239 \\
0.2384 \\
0.4193 \\
0.3975 \\
0.4286\end{array}$ & $\begin{array}{l}0.098 \\
0.098 \\
0.098 \\
0.099 \\
0.099 \\
0.099 \\
0.099 \\
0.099 \\
0.099 \\
0.096 \\
0.096 \\
0.096 \\
0.101 \\
0.101 \\
0.101\end{array}$ & $\begin{array}{l}18.8 \\
19.0 \\
17.7 \\
24.6 \\
25.0 \\
24.6 \\
25.8 \\
26.2 \\
23.8 \\
29.4 \\
29.2 \\
30.3 \\
44.5 \\
42.9 \\
45.5\end{array}$ \\
\hline
\end{tabular}

Tabelle A 2: Versuchsdaten

Bei den im Versuchsbericht beschriebenen Versuchen ohne Umschnürungsbewehrung sind nur $88 \%$ der an Zylindern $\left(d=152, l=254 \mathrm{~mm}\right.$ ) gemessenen Festigkeit $f_{c}$ erreicht worden (size effect?). Diese Festigkeitsabnahme ist bei der in Tabelle $\mathrm{A} 2$ angegebenen Festigkeit berücksichtigt worden.

Die Resultate dieser Auswertung sind im Bild 4.3c dargestellt. 


\section{Anhang 3: Auswertung der Versuche von Somes (1970) an prismatischen Stützenelementen mit Umschnürungsbewehrung}

Die Probekörper waren $305 \mathrm{~mm}$ lang und hatten eine Flăche von $102 \times 102 \mathrm{~mm}$. Die Querbewehrung bestand aus quadratischen Rohrabschnitten. Sie hatten keine Betonüberdeckung. Die Versuchsdaten und die Resultate sind in der Tabelle A 3 zusammengestellt. Die Betonfestigkeit $f_{c}$ wurde an unbewehrten Probekörpern gleicher Abmessungen bestimmt. Die Resultate dieser Auswertung sind im Bild 4.5c dargestellt.

\begin{tabular}{|c|c|c|c|c|c|c|c|c|c|}
\hline Versuch & $d$ & $8^{\circ}$ & $A=t$ & & fe & $f=y$ & & $\approx / d$ & 103 \\
\hline $\begin{array}{l}011 \\
012 \\
013 \\
014 \\
015 \\
016 \\
c 17 \\
021 \\
c 22 \\
023 \\
024 \\
c 25 \\
026 \\
027 \\
b 11 \\
b 12 \\
b 13 \\
b 14 \\
b 15 \\
b 16 \\
b 17 \\
b 21 \\
b 22 \\
b 23 \\
b 24 \\
b 25 \\
b 26 \\
b 27 \\
c 11 \\
c 12 \\
c 13 \\
c 14 \\
c 15 \\
c 16 \\
c 17 \\
c 21 \\
c 22 \\
c 23 \\
c 24 \\
c 25 \\
c 26 \\
c 27\end{array}$ & $\begin{array}{l}102 . \\
102 . \\
102 . \\
102 . \\
102 . \\
102 . \\
102 . \\
102 . \\
102 . \\
102 . \\
102 . \\
102 . \\
102 . \\
102 . \\
102 . \\
102 . \\
102 . \\
102 . \\
102 . \\
102 . \\
102 . \\
102 . \\
102 . \\
102 . \\
102 . \\
102 . \\
102 . \\
102 . \\
102 . \\
102 . \\
102 . \\
102 . \\
102 . \\
102 . \\
102 . \\
102 . \\
102 . \\
102 . \\
102 . \\
102 . \\
102 . \\
102 .\end{array}$. & $\begin{array}{l}19 . \\
25 . \\
32 . \\
38 . \\
44 . \\
51 . \\
57 . \\
64 . \\
76 . \\
89 . \\
102 . \\
114 . \\
140 . \\
152 . \\
19 . \\
25 . \\
32 . \\
38 . \\
44 . \\
51 . \\
57 . \\
64 . \\
76 . \\
89 . \\
102 . \\
114 . \\
140 . \\
152 . \\
19 . \\
25 . \\
32 . \\
38 . \\
44 . \\
51 . \\
57 . \\
64 . \\
76 . \\
89 . \\
102 . \\
114 . \\
140 . \\
152 .\end{array}$ & $\begin{array}{l}41 . \\
41 . \\
41 . \\
41 . \\
41 . \\
41 . \\
41 . \\
41 . \\
41 . \\
41 . \\
41 . \\
41 . \\
41 . \\
41 . \\
30 . \\
30 . \\
30 . \\
30 . \\
30 . \\
30 . \\
30 . \\
30 . \\
30 . \\
30 . \\
30 . \\
30 . \\
30 . \\
30 . \\
27 . \\
27 . \\
27 . \\
27 . \\
27 . \\
27 . \\
27 . \\
27 . \\
27 . \\
27 . \\
27 . \\
27 . \\
27 . \\
27 . \\
.5\end{array}$ & $\begin{array}{l}0.0423 \\
0.0318 \\
0.0254 \\
0.0212 \\
0.0181 \\
0.0159 \\
0.0141 \\
0.0127 \\
0.0106 \\
0.0091 \\
0.0079 \\
0.0071 \\
0.0058 \\
0.0053 \\
0.0307 \\
0.0230 \\
0.0184 \\
0.0153 \\
0.0131 \\
0.0115 \\
0.0102 \\
0.0092 \\
0.0077 \\
0.0066 \\
0.0058 \\
0.0051 \\
0.0042 \\
0.0038 \\
0.0277 \\
0.0207 \\
0.0166 \\
0.0138 \\
0.0119 \\
0.0104 \\
0.0092 \\
0.0083 \\
0.0069 \\
0.0059 \\
0.0052 \\
0.0046 \\
0.0038 \\
0.0035\end{array}$ & $\begin{array}{l}36.5 \\
36.5 \\
36.5 \\
36.5 \\
36.5 \\
36.5 \\
36.5 \\
31.4 \\
31.4 \\
31.4 \\
31.4 \\
31.4 \\
31.4 \\
31.4 \\
28.5 \\
28.5 \\
28.5 \\
28.5 \\
28.5 \\
28.5 \\
28.5 \\
29.6 \\
29.6 \\
29.6 \\
29.6 \\
29.6 \\
29.6 \\
29.6 \\
30.3 \\
30.3 \\
30.3 \\
30.3 \\
30.3 \\
30.3 \\
30.3 \\
33.9 \\
33.9 \\
33.9 \\
33.9 \\
33.9 \\
33.9\end{array}$ & $\begin{array}{l}324 . \\
324 . \\
324 . \\
324 . \\
324 . \\
324 . \\
324 . \\
324 . \\
324 . \\
324 . \\
324 . \\
324 . \\
324 . \\
324 . \\
324 . \\
324 . \\
324 . \\
324 . \\
324 . \\
324 . \\
324 . \\
324 . \\
324 . \\
324 . \\
324 . \\
324 . \\
324 . \\
324 . \\
324 . \\
324 . \\
324 . \\
324 . \\
324 . \\
324 . \\
324 . \\
324 . \\
324 . \\
324 . \\
324 . \\
324 . \\
324 . \\
324 .\end{array}$ & $\begin{array}{l}0.3753 \\
0.2815 \\
0.2252 \\
0.1877 \\
0.1609 \\
0.1408 \\
0.1251 \\
0.1312 \\
0.1093 \\
0.0937 \\
0.0820 \\
0.0729 \\
0.0596 \\
0.0547 \\
0.3489 \\
0.2617 \\
0.2094 \\
0.1745 \\
0.1495 \\
0.1308 \\
0.1163 \\
0.1005 \\
0.0838 \\
0.0718 \\
0.0628 \\
0.0559 \\
0.0457 \\
0.0419 \\
0.2961 \\
0.2221 \\
0.1777 \\
0.1481 \\
0.1269 \\
0.1111 \\
0.0987 \\
0.0793 \\
0.0661 \\
0.0566 \\
0.0495 \\
0.0440 \\
0.0360 \\
0.0330\end{array}$ & $\begin{array}{l}0.188 \\
0.250 \\
0.313 \\
0.375 \\
0.438 \\
0.500 \\
0.563 \\
0.625 \\
0.750 \\
0.875 \\
1.000 \\
1.125 \\
1.375 \\
1.500 \\
0.188 \\
0.250 \\
0.313 \\
0.375 \\
0.438 \\
0.500 \\
0.563 \\
0.625 \\
0.750 \\
0.875 \\
1.000 \\
1.125 \\
1.375 \\
1.500 \\
0.188 \\
0.250 \\
0.313 \\
0.375 \\
0.438 \\
0.500 \\
0.563 \\
0.625 \\
0.750 \\
0.875 \\
1.000 \\
1.125 \\
1.375 \\
1.500\end{array}$ & $\begin{array}{l}63.1 \\
55.3 \\
52.2 \\
50.1 \\
44.7 \\
45.5 \\
46.0 \\
41.7 \\
39.9 \\
37.4 \\
37.8 \\
37.0 \\
35.2 \\
32.6 \\
53.4 \\
46.7 \\
41.8 \\
39.8 \\
38.3 \\
35.9 \\
36.4 \\
39.5 \\
37.5 \\
35.9 \\
34.2 \\
33.6 \\
31.6 \\
30.2 \\
45.4 \\
43.0 \\
40.4 \\
39.9 \\
38.8 \\
37.2 \\
36.3 \\
37.3 \\
37.0 \\
35.9 \\
35.1 \\
35.5 \\
34.6 \\
33.7\end{array}$ \\
\hline
\end{tabular}

Tabelle A 3: Versuchsresultate 


\section{Anhang A4: Auswertung der Versuche von Sozen, Zwoyer, Siess (1959)} an vorgespannten Balken ohne Schubbewehrung

Die hier ausgewerteten Versuche hatten die gleichen Betonabmessungen und waren gleich bewehrt:

$$
\begin{aligned}
& a=914 \mathrm{~mm} \\
& d=208 \mathrm{~mm} \\
& t=152 \mathrm{~mm} \\
& A_{S}=246 \mathrm{~mm}^{2} \\
& f_{p y}=1434 \mathrm{MPa}
\end{aligned}
$$

Die Variablen (Betonfestigkeit $t_{c}$ und Vorspannkraft $P$ ) sowie die Resultate sind in der folgenden Tabelle zusammengestellt.

\begin{tabular}{|l|l|l|r|l|l|l|}
\hline Versuch & $V$ & $f_{c}$ & $P$ & $V / N_{\text {flex }}$ & $P / V$ & $V / t \cdot d \cdot f_{c}$ \\
\hline A 3237 & 38942. & 42.2 & 8474. & 0.559 & 0.22 & 0.029 \\
A 3249 & 46482. & 32.8 & 57624. & 0.697 & 1.24 & 0.045 \\
A 2249 & 50996. & 32.8 & 96266. & 0.764 & 1.89 & 0.049 \\
A 2240 & 58691. & 39.9 & 122028 & 0.849 & 2.08 & 0.046 \\
A 1248 & 66609. & 33.4 & 237277. & 0.995 & 3.56 & 0.063. \\
\hline
\end{tabular}

Tabelle A4: Versuchsdaten

Die Biegetraglast $V_{\text {flex }}$ ist mit der Plastizitătstheorie berechnet worden (konstante Spannungsintensität $f_{c}$ in der Druckzone).

Die Resultate dieser Auswertung sind im Bild 6.7 dargestellt. 


\section{Anhang A5: Auswertung der Versuche von Tomii, Sakino, Kiyohara (1981) an unbewehrten Stützenelementen}

Die Betonabmessungen und die Beanspruchungsart sind im Bild A5 dargestellt. Die Einspannblöcke an beiden Enden der Probekörper waren so befestigt, dass horizontale und vertikale Relativverschiebungen, jedoch keine Relativverdrehungen möglich waren.

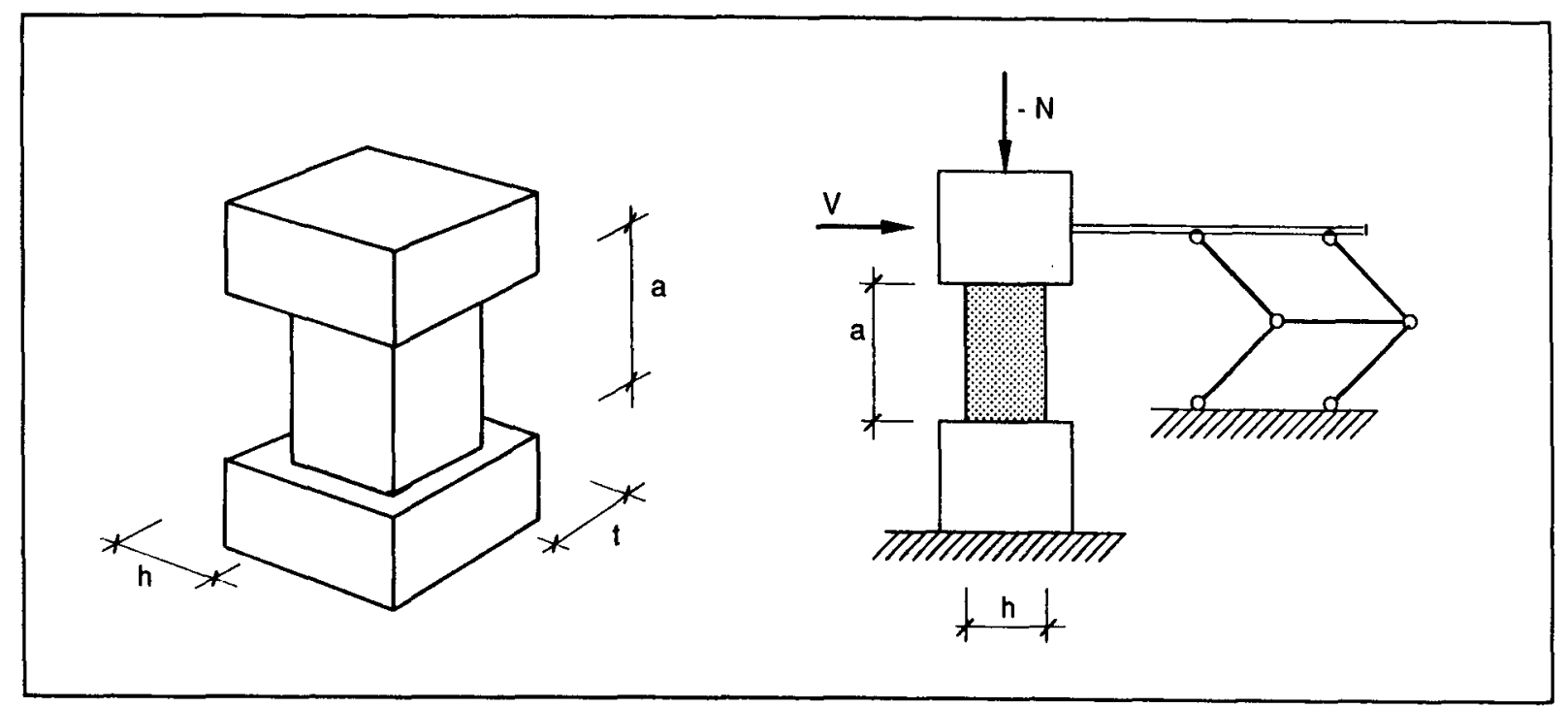

Bild A5: Abmessungen der Probekörper und Beanspruchungsart

Variert wurden die Spannweite a, die Normalkraft $N$ und die Betonfestigkeit $f_{c}$. Die Betonfestigkeit wurde an Zylindern $d=100 \mathrm{~mm}, l=200 \mathrm{~mm}$ gemessen. Die Versuchsdaten und die Resultate sind in der folgenden Tabelle zusammengestellt. Die Resultate dieser Auswertung sindn im Bild 6.11 graphisch dargestellt. 
$.157-$

\begin{tabular}{|c|c|c|c|c|c|c|}
\hline Versuchserie & $\begin{array}{l}n \\
\mathrm{~mm}\end{array}$ & $\mathrm{t}$ & $\begin{array}{l}0 \\
\mathrm{~mm}\end{array}$ & $\begin{array}{l}f C \\
\text { MPo }\end{array}$ & $\frac{-N}{\operatorname{th} t c}$ & $\frac{V}{t h+c}$ \\
\hline 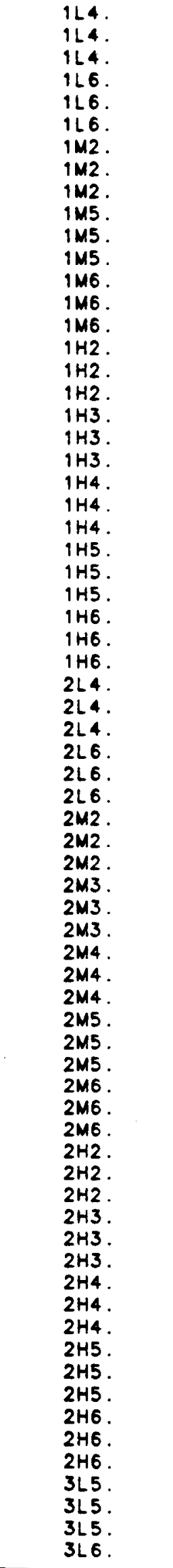 & 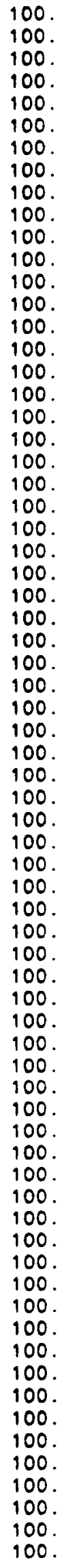 & 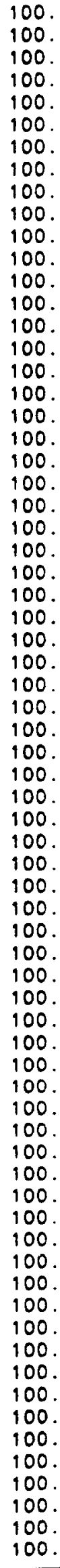 & 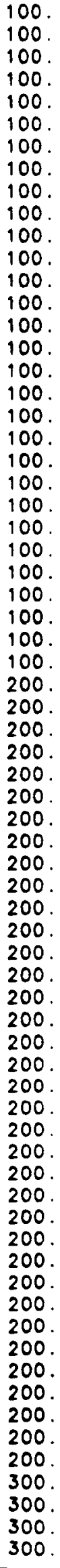 & 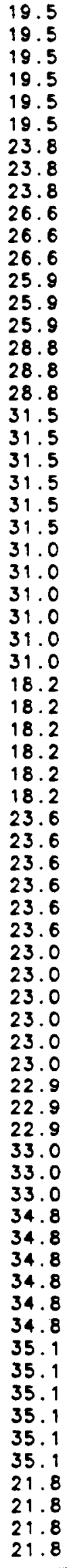 & 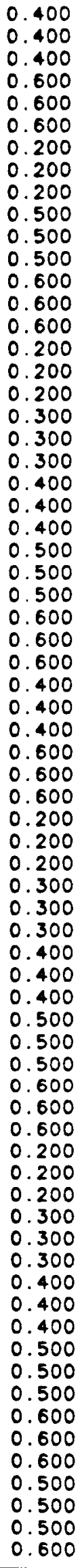 & 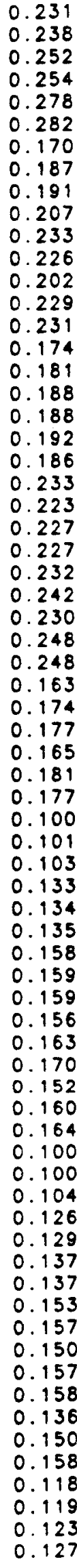 \\
\hline
\end{tabular}

Tabelle A5: Versuchsdaten und Resultate 


\begin{tabular}{|c|c|c|c|c|c|c|}
\hline Versuchserie & $\begin{array}{l}h \\
m m\end{array}$ & $\begin{array}{l}t \\
m m\end{array}$ & $\begin{array}{l}0 \\
\mathrm{~mm}\end{array}$ & $\begin{array}{l}f C \\
M P O\end{array}$ & $\frac{-N}{\text { thic }}$ & $\frac{v}{\operatorname{th} f c}$ \\
\hline $\begin{array}{l}3 L 6 . \\
3 L 6 . \\
3 M 2 . \\
3 M 2 . \\
3 M 2 . \\
3 M 3 . \\
3 M 3 . \\
3 M 3 . \\
3 M 4 . \\
3 M 4 . \\
3 M 4 . \\
3 M 5 . \\
3 M 5 . \\
3 M 5 . \\
3 H 2 . \\
3 H 2 . \\
3 H 2 . \\
3 H 3 . \\
3 H 3 . \\
3 H 3 . \\
3 H 4 . \\
3 H 4 . \\
3 H 4 . \\
3 H 5 . \\
3 H 5 . \\
3 H 5 . \\
3 H 6 . \\
3 H 6 . \\
3 H 6 .\end{array}$ & $\begin{array}{l}100 . \\
100 . \\
100 . \\
100 . \\
100 . \\
100 . \\
100 . \\
100 . \\
100 . \\
100 . \\
100 . \\
100 . \\
100 . \\
100 . \\
100 . \\
100 . \\
100 . \\
100 . \\
100 . \\
100 . \\
100 . \\
100 . \\
100 . \\
100 . \\
100 . \\
100 . \\
100 . \\
100 . \\
100 .\end{array}$ & $\begin{array}{l}100 . \\
100 . \\
100 . \\
100 . \\
100 . \\
100 . \\
100 . \\
100 . \\
100 . \\
100 . \\
100 . \\
100 . \\
100 . \\
100 . \\
100 . \\
100 . \\
100 . \\
100 . \\
100 . \\
100 . \\
100 . \\
100 . \\
100 . \\
100 . \\
100 . \\
100 . \\
100 . \\
100 . \\
100 .\end{array}$ & $\begin{array}{l}300 . \\
300 . \\
300 . \\
300 . \\
300 . \\
300 . \\
300 . \\
300 . \\
300 . \\
300 . \\
300 . \\
300 . \\
300 . \\
300 . \\
300 . \\
300 . \\
300 . \\
300 . \\
300 . \\
300 . \\
300 . \\
300 . \\
300 . \\
300 . \\
300 . \\
300 . \\
300 . \\
300 . \\
300 .\end{array}$ & $\begin{array}{l}21.8 \\
21.8 \\
23.9 \\
23.9 \\
23.9 \\
23.9 \\
23.9 \\
23.9 \\
23.7 \\
23.7 \\
23.7 \\
23.7 \\
23.7 \\
23.7 \\
32.3 \\
32.3 \\
32.3 \\
32.3 \\
32.3 \\
32.3 \\
31.7 \\
31.7 \\
31.7 \\
31.7 \\
31.7 \\
31.7 \\
31.3 \\
31.3 \\
31.3\end{array}$ & $\begin{array}{l}0.600 \\
0.600 \\
0.200 \\
0.200 \\
0.200 \\
0.300 \\
0.300 \\
0.300 \\
0.400 \\
0.400 \\
0.400 \\
0.500 \\
0.500 \\
0.500 \\
0.200 \\
0.200 \\
0.200 \\
0.300 \\
0.300 \\
0.300 \\
0.400 \\
0.400 \\
0.400 \\
0.500 \\
0.500 \\
0.500 \\
0.600 \\
0.600 \\
0.600\end{array}$ & $\begin{array}{l}0.131 \\
0.137 \\
0.061 \\
0.062 \\
0.064 \\
0.083 \\
0.085 \\
0.085 \\
0.101 \\
0.103 \\
0.106 \\
0.109 \\
0.111 \\
0.115 \\
0.065 \\
0.063 \\
0.062 \\
0.085 \\
0.085 \\
0.085 \\
0.102 \\
0.105 \\
0.106 \\
0.116 \\
0.121 \\
0.126 \\
0.109 \\
0.121 \\
0.128\end{array}$ \\
\hline
\end{tabular}

Tabelle A5: Versuchsdaten und Resultate (Fortsetzung) 